\title{
An Exploration of the Factors Influencing Home Users' Cybersecurity Behaviours \\ by
}

Nicole Kristine Henika Braun

A thesis submitted to Victoria University of Wellington in fulfilment of the requirements for the degree of Doctor of Philosophy

Victoria University of Wellington 2014 





\section{Abstract}

Cybersecurity has been a concern for businesses and governments since their initial uptake of the Internet in the 1970s. As more and more people started using the internet for personal use, cybersecurity has become an important concern for home users as well. However, most research on cybersecurity has been undertaken at an organisational rather than at the individual level. Individual behaviours online have became increasingly important as the line between home and business use has blurred and users' actions on their home computers has begun to have more wide ranging implications. There appears to be a lack of agreement on how to approach the topic of internet security outside of an organisational perspective.

This research focuses on the individual home user perspective and seeks to (1) identify the factors that relate to home users' security behaviours, and (2) examine how the identified factors relate to users' cybersecurity behaviours. A conceptual framework was developed based on the literature to guide the data collection.

To identify the relevant factors relating to home users' security behaviours, a qualitative study comprised of three focus groups and 20 individual interviews was carried out. From the data, a revised model was developed. In the revised model, awareness of threats was identified as a necessary first cybersecurity step before users can form opinions about the danger of threats. Awareness of threats was influenced by users' characteristics, opinions, and experiences as well as by factors in their external environments.

The combination of internal factors, external factors, and awareness led to users' perceptions about the danger of threats online. This perception of danger led to users' intentions to engage in protective behaviours. However, these intentions were strengthened or weakened based on users' perceptions about the barriers to and enablers of security.

By applying the revised model, it was possible to identify different types of security users. Through the identification of these user types, eight factors emerged as being particularly important in influencing users' perceptions of threats and dangers: knowledge, perceived self-efficacy, trust, threat awareness, safeguard awareness, prior experience, reliance, and security orientation. These factors from the model are used as a starting point to understanding how users make decisions about what they will do to protect themselves online. Further, through the identification of these user types suggestions are made about how to promote security for different types of individuals. 


\section{Acknowledgements}

I would like to acknowledge the contributions of the people who have helped me in my journey through the $\mathrm{PhD}$ process. I would like to thank my supervisors, Dr. Dan Dorner and Dr. Val Hooper who have provided their support, guidance and suggestions throughout the process. I would also like to thank Professor Pak Yoong who first encouraged me to do my PhD. A further thank you goes to Dr. Jocelyn Cranefield who provided help and advice when I needed it most. My sincere thank you as well to the academic and administrative staff at the School of Information Management at Victoria University of Wellington.

I would also like to thank my family and friends who have supported me. My parents, Jim and Kathy, have always supported me in whatever I wanted to do, even if it was studying half way around the world. Their support provided me with the strength to keep going even when things were difficult. Peter Monk has been there for me throughout the process. I cannot thank him enough for his support. To my friends, Nishanie, Balsam, Sai, and Fahimi, I thank you for your encouragement and the smiles along the way.

Finally, I could not have done this research without the help of my respondents. Their participation made this research possible and their responses provided me with valuable insight into a topic that I have thoroughly enjoyed studying.

To everyone else that I haven't mentioned by name, know that I appreciate your help along the way. Although writing a $\mathrm{PhD}$ feels like a very solitary process at times, it was the help of those around me that helped keep me motivated to keep going with it. 


\section{Table of Contents}

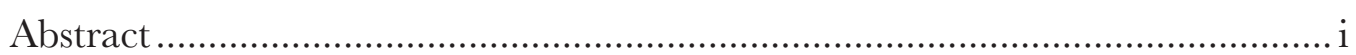

Acknowledgements ......................................................................................... ii

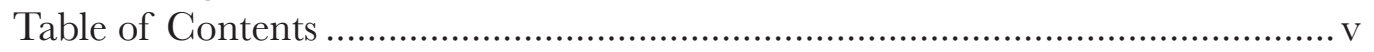

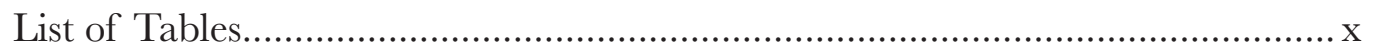

List of Figures ..............................................................................................

List of Abbreviations................................................................................. xiv

\section{Chapter 1 Introduction}

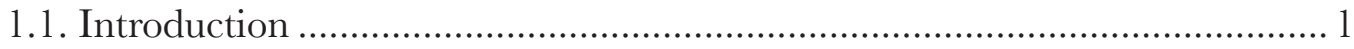

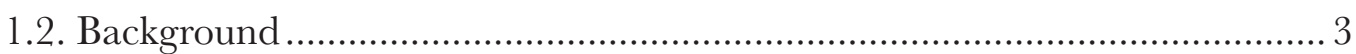

1.2.1. Cybersecurity Threat Landscape ................................................... 3

1.2.2. Cybersecurity Safeguards ......................................................... 5

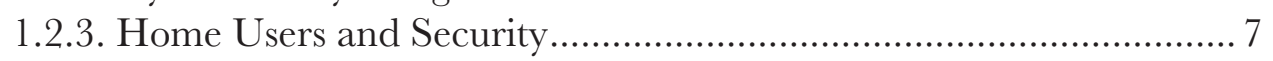

1.2.4. Encouraging Cybersecurity Behaviours ......................................... 8

1.3. Research Problem, Questions, and Objectives .............................................. 10

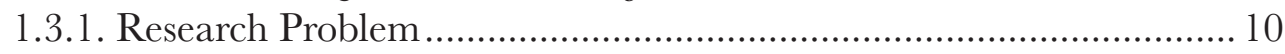

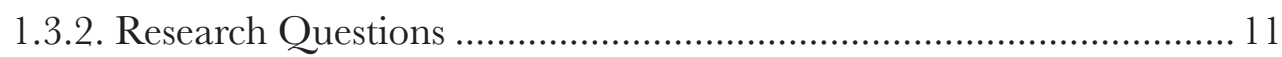

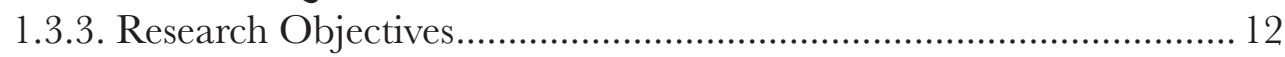

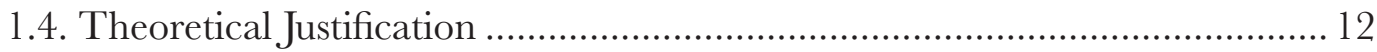

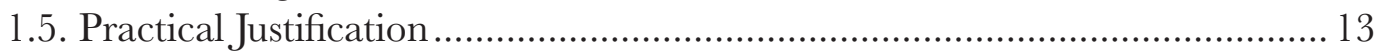

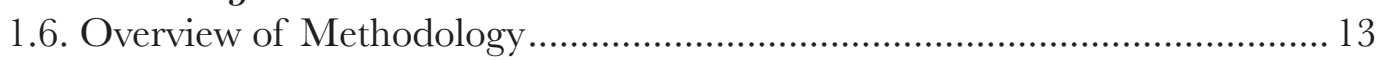

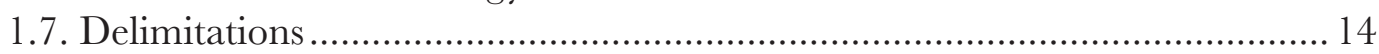

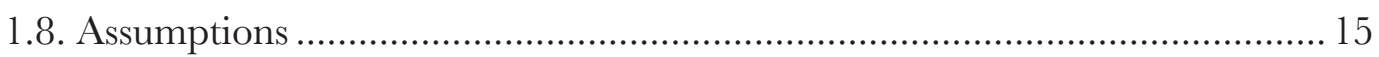

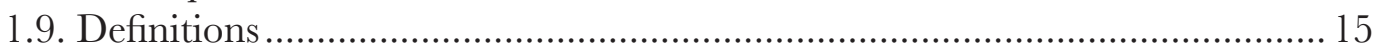

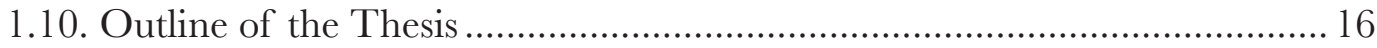

\section{Chapter 2 Literature Review}

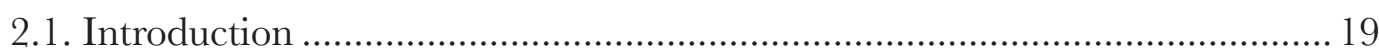

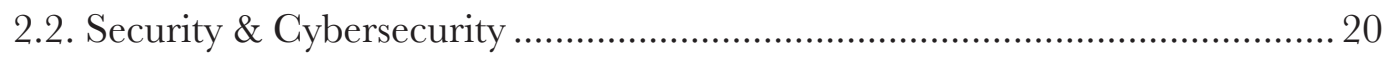

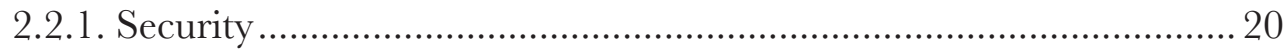

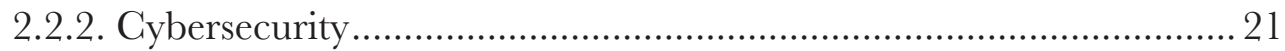

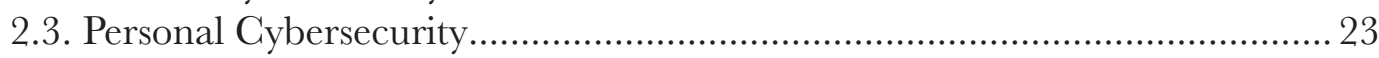

2.3.1. Home User ............................................................................. 25

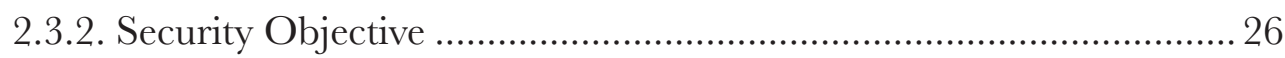

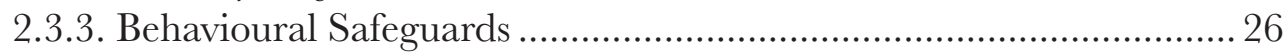

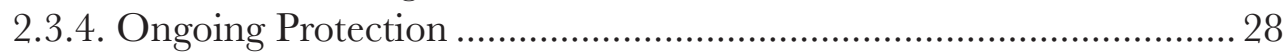

2.3.5. Ongoing Home User Security Behaviours ....................................... 29

2.4. Proposed Factors Associated with Cybersecurity Behaviours ......................... 30

2.4.1. Perceived Threat and Perceived Risk ............................................... 32

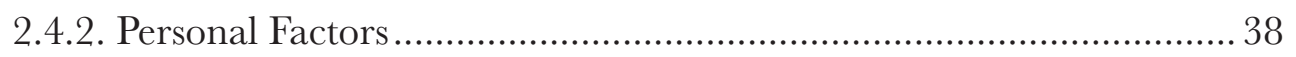




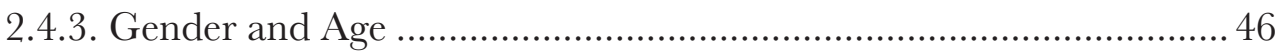

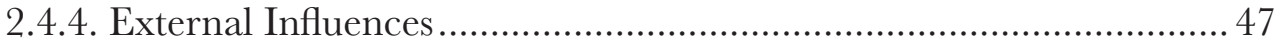

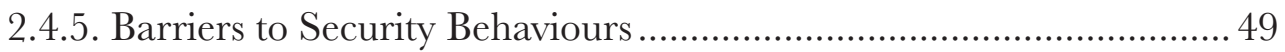

2.5. Summary of the Literature \& Research Gap ............................................... 50

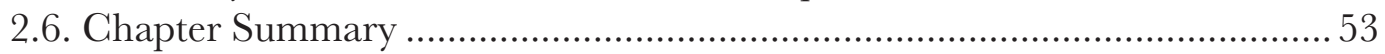

\section{Chapter 3 Methodology}

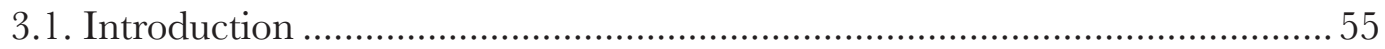

3.2. Overview of Paradigm, Approach, and Methods....................................... 55

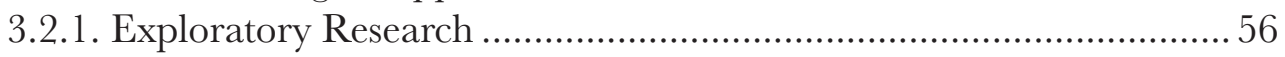

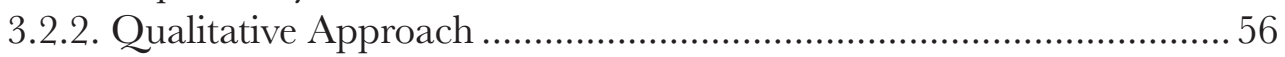

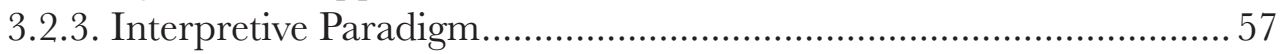

3.2.4. Use of Focus Groups and Interviews............................................... 58

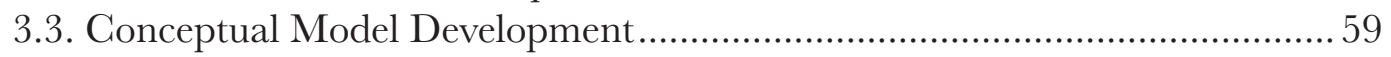

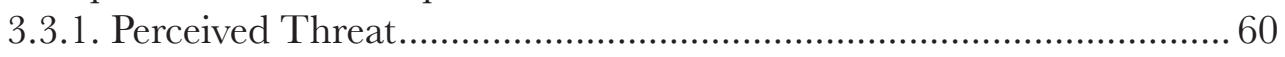

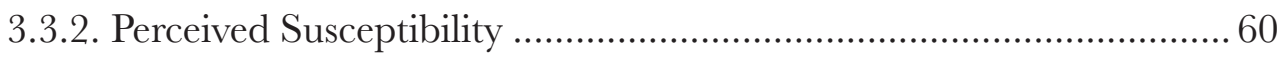

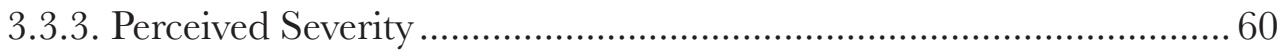

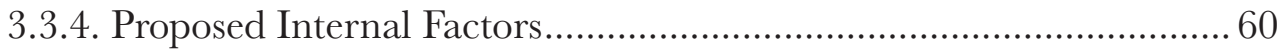

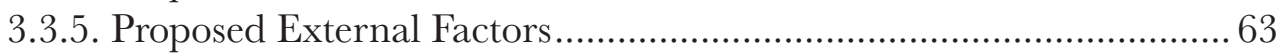

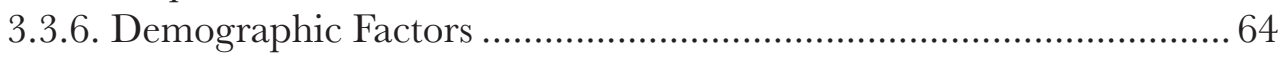

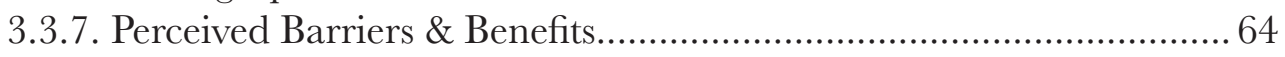

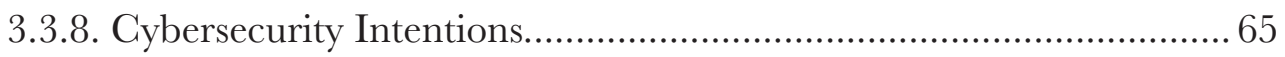

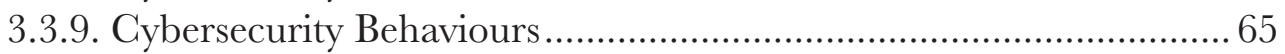

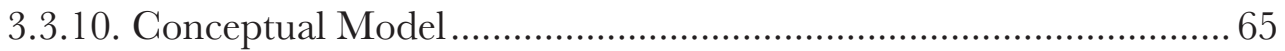

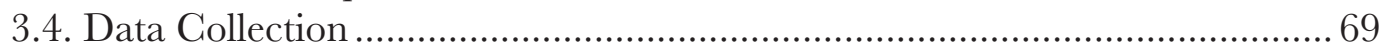

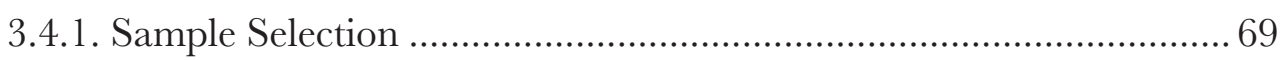

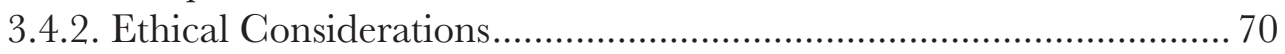

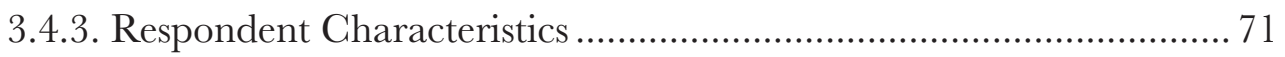

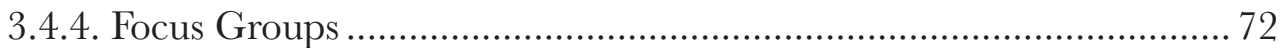

3.4.5. Individual Interviews ................................................................... 73

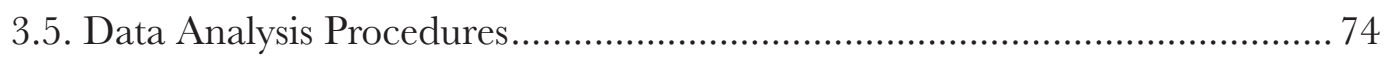

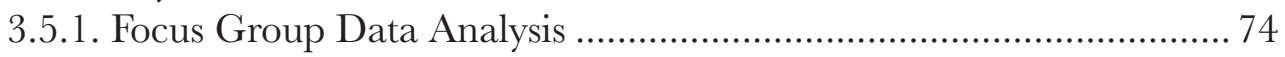

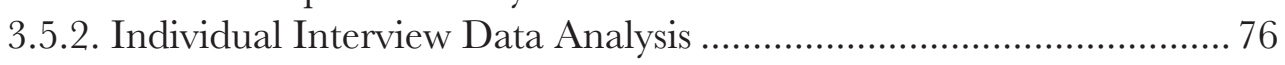

3.5.3. Integration of Focus Group and Individual Interview Data ............ 78

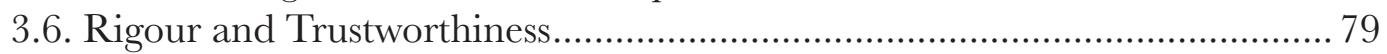

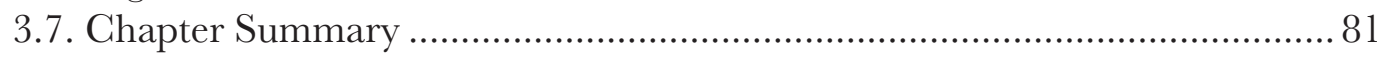

\section{Chapter 4 Findings}

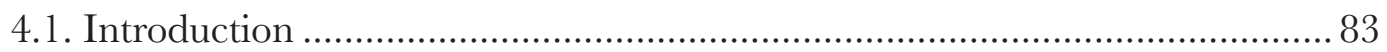

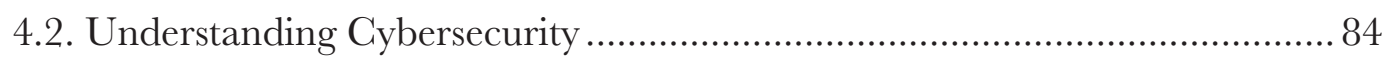

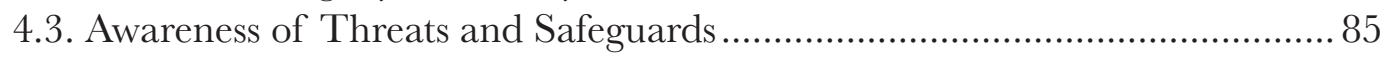

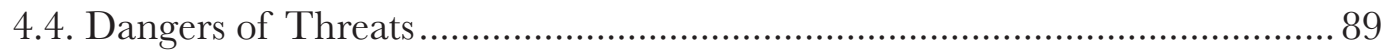

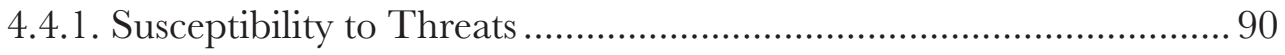

vi

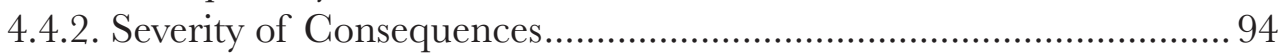




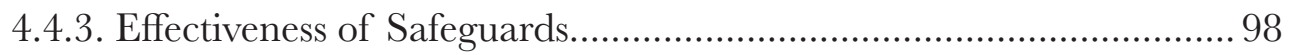

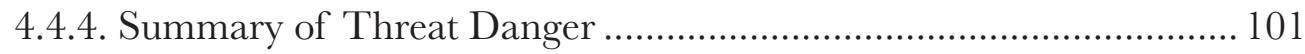

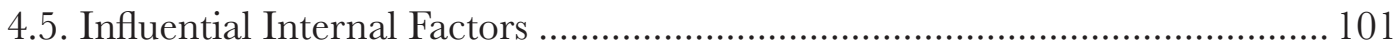

4.5.1. Past Experiences with Breaches..................................................... 102

4.5.2. Knowledge of Threats and Safeguards ........................................... 106

4.5.3. Perceived Self-Efficacy and Confidence ........................................... 111

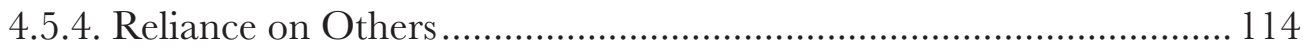

4.5.5. Trust in Websites and People ......................................................... 116

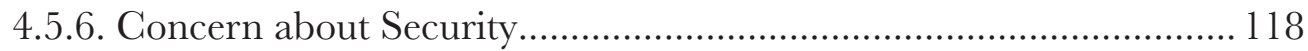

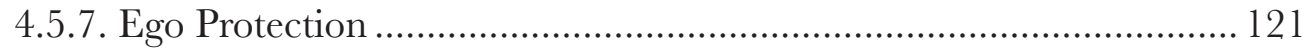

4.5.8. Summary of Internal Factors ......................................................... 122

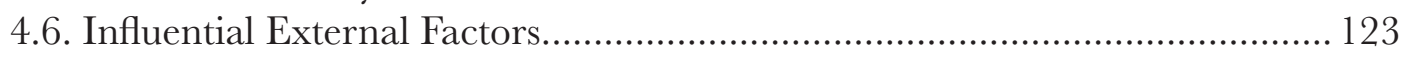

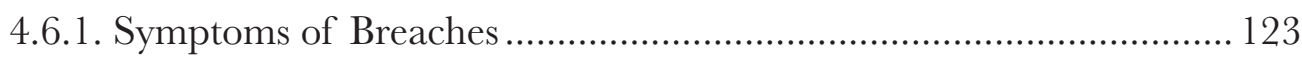

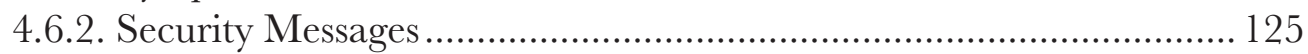

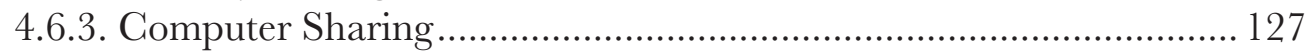

4.6.4. Summary of External Factors .......................................................... 131

4.7. Perceived Barriers \& Enablers...................................................................... 132

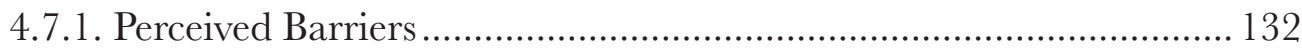

4.7.2. Perceived Enablers............................................................................. 136

4.7.3. Summary of Perceived Barriers \& Enablers....................................... 138

4.8. Cybersecurity Intentions \& Behaviours ........................................................ 138

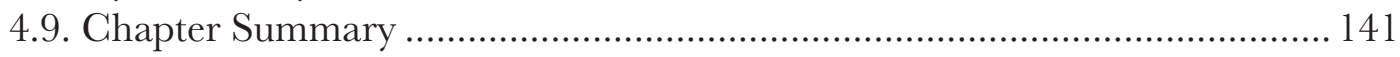

\section{Chapter 5 Revised Model}

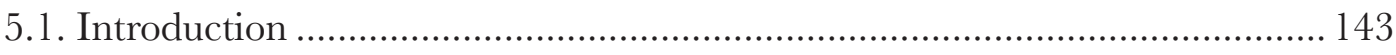

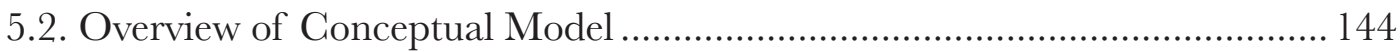

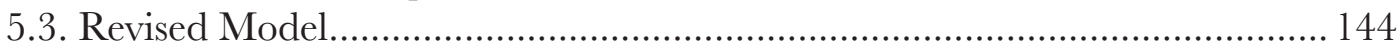

5.3.1. Definitions of Revised Model Components .................................... 145

5.3.2. Key Changes to the Conceptual Model .......................................... 150

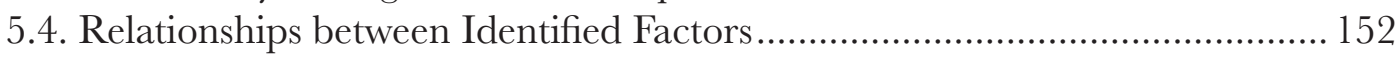

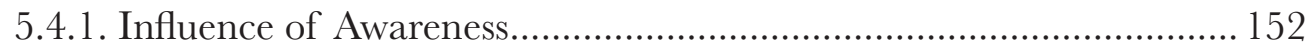

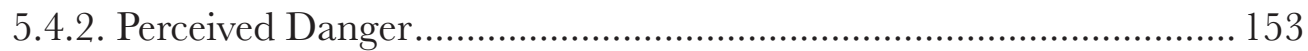

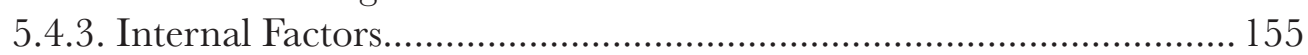

5.4.4. External Factors........................................................................... 170

5.4.5. Perceived Barriers and Enablers .................................................... 179

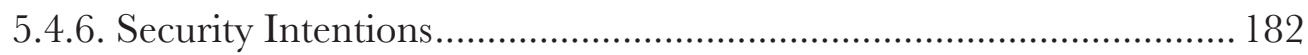

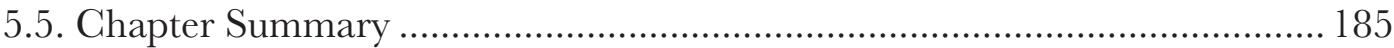

\section{Chapter 6 User Types}

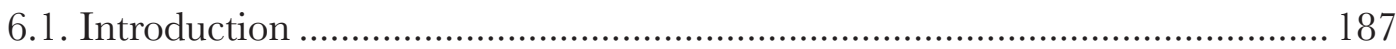

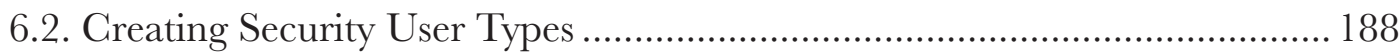

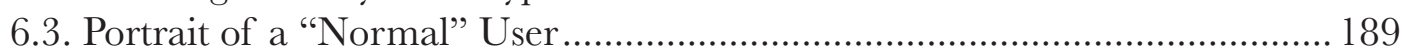

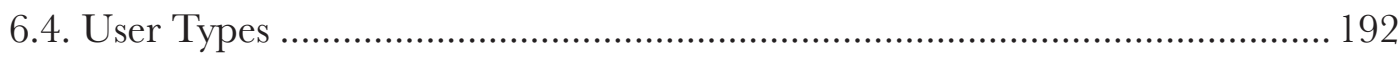




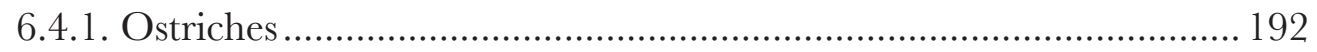

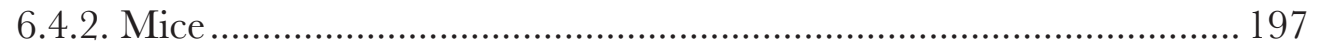

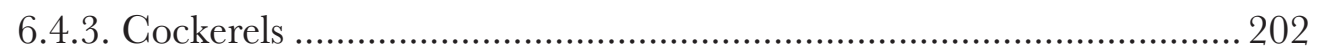

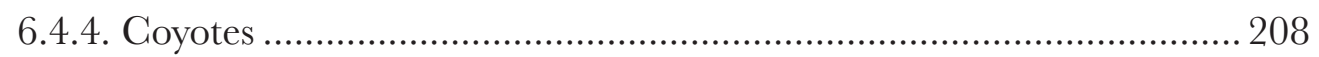

6.4.5. Dark Horses ........................................................................... 212

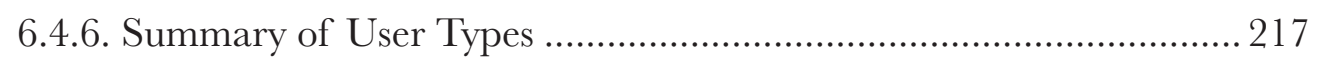

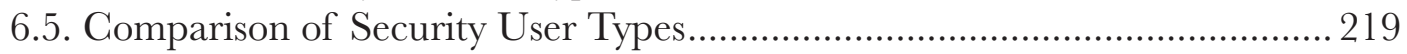

6.5.1. The Average Respondent and the Perceived Normal User.............. 219

6.5.2. Comparison of User Types Based on Perceived Self-Efficacy........... 221

6.5.3. Comparison of User Types based on Knowledge ........................... 222

6.5.4. Most and Least Protective Behaviours by User Types...................... 223

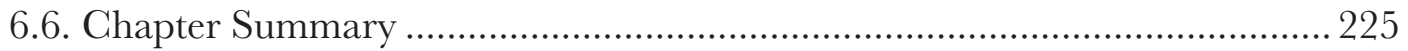

\section{Chapter 7 Discussion}

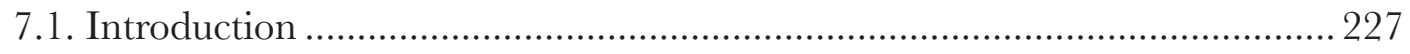

7.2. Key Aspects Influencing Users' Security Intentions .................................. 228

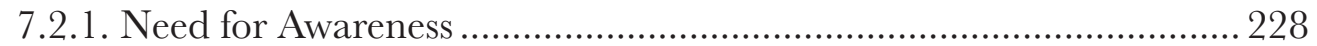

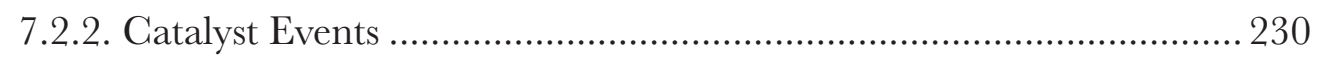

7.2.3. Knowledge and Perceived Self-Efficacy ....................................... 232

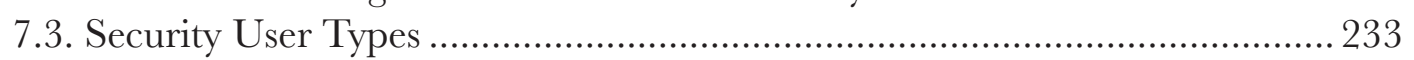

7.3.1. Comparison to Existing User Types ................................................ 234

7.3.2. Recommendations for Safer Behaviours by User Type .................... 237

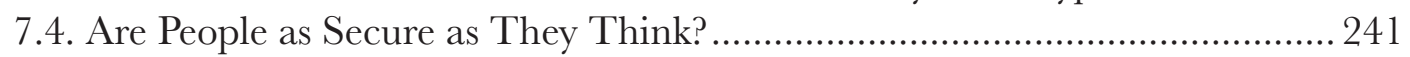

7.5. Why Are People Lazy About Security? ....................................................... 242

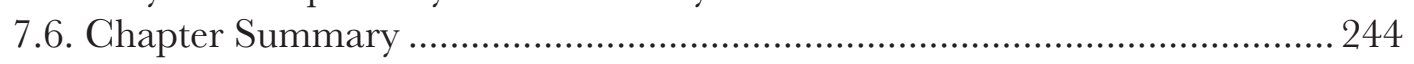

\section{Chapter 8 Conclusions}

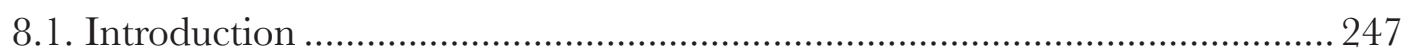

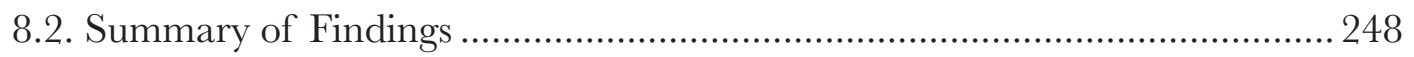

8.2.1. Factors Influencing Home Users' Cybersecurity Intentions.............. 249

8.2.2. Relationships between Factors Influencing Home Users' Cybersecurity Intentions............................................................. 251

8.2.3. The Gap between Awareness and Practice ..................................... 256

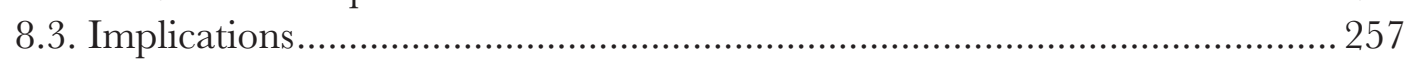

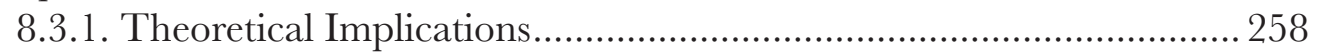

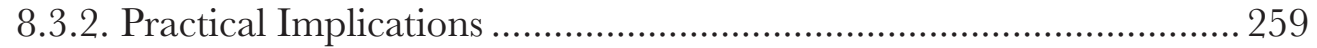

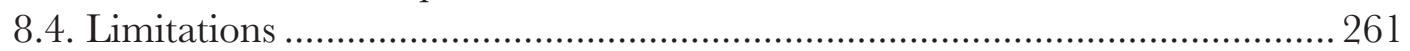

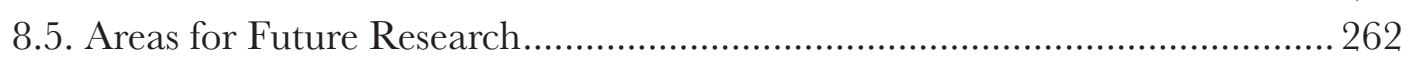

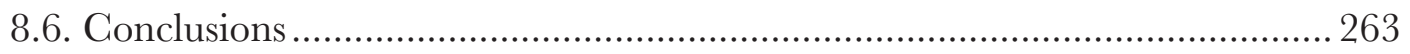

\section{Chapter 9 References}




\section{Appendices}

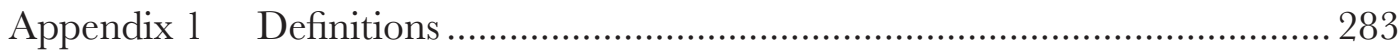

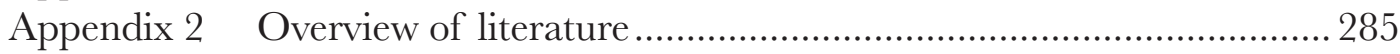

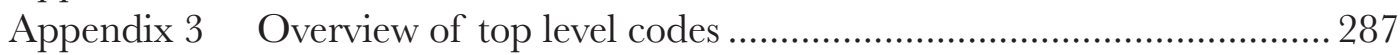

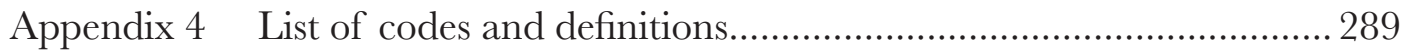

Appendix 5 Mind map of initial relationships between codes.......................... 325

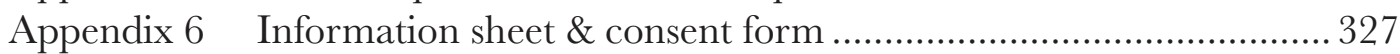

Appendix 7 Explanation of ranking used in the coding process ...................... 333 


\section{List of Tables}

Table 2-1. Definitions and descriptions relating to components of

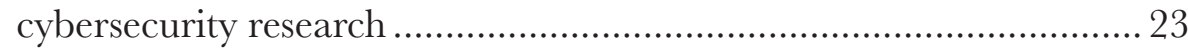

Table 2-2. Factors influencing cybersecurity and online privacy intentions .......27

Table 2-3. Theories used in cybersecurity and other relevant research .............. 30

Table 2-4. Identification of main factors from theories that have been used in cybersecurity research ............................................................ 31

Table 2-5. Summary of factors examined in previous literature relevant to users' cybersecurity behaviours .................................................... 51

Table 3-1. Description of concepts and identification of components in the conceptual model

Table 3-2. Example of how codes were grouped in the second stage of the

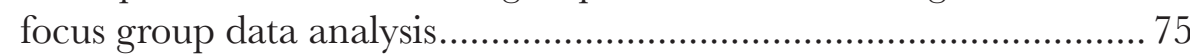

Table 3-3. Example of coding used in the third stage of coding the focus

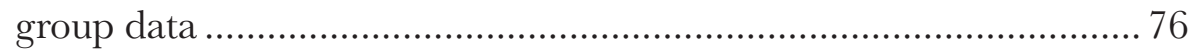

Table 3-4. Steps taken to resolve duplicate and additional codes from the focus groups and individual interviews ........................................... 78

Table 4-1. Terms associated with interviewees' definitions of cybersecurity and internet security.

Table 4-2. Top ranked threats participants were concerned about, in descending order.... 86

Table 4-3. Comparison of focus groups' top ranked threats with interviewees' most frequently mentioned threats....

Table 4-4. Respondent comments illustrating varying levels of cybersecurity threat awareness

Table 4-5. Respondent comments illustrating varying levels of perceived susceptibility to cybersecurity threats

Table 4-6. Respondent descriptions of malicious users, reasons for malicious users to engage in malicious behaviours, and the outcomes malicious users' hoped to accomplish

Table 4-7. Respondent comments illustrating varying levels of perceived severity of consequences of cybersecurity threats.

Table 4-8. Respondent comments about whether they had experienced a cybersecurity breach in the past.

Table 4-9. Respondent comments on their knowledge of cybersecurity threats

Table 4-10. Examples of respondent comments on their knowledge of cybersecurity safeguards.

Table 4-11. Most frequently cited sources of information (in descending order), by gender

Table 4-12. Summary of computer sharing behaviours based on security response types (active, passive, or combination) and when those steps were taken.

Table 4-13. Top ranked barriers to engaging in cybersecurity barriers (in descending order)

Table 4-14. Top ranked reasons to engage in cybersecurity behaviours (in descending order) 
Table 5-1. Summary and definitions of the factors in the revised model and the associated components.

Table 5-2. Summary of changes to the relationships between the factors in the conceptual model and revised model....................................... 151

Table 6-1. Summary of user type characteristics and behaviours...................... 218

Table 7-1. Summary of user types and clusters related to internet security

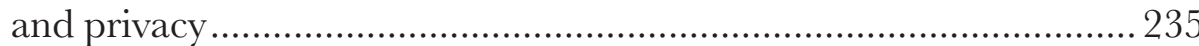

Table 7-2. Summary of challenges and recommendations by user type............. 238 


\section{List of Figures}

Figure 2-1. Detailed analysis of the components of cybersecurity research ........ 24

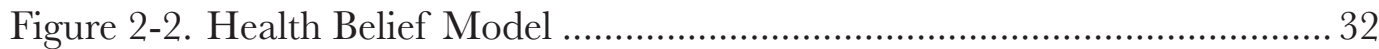

Figure 2-3. Protection Motivation Theory .......................................................... 33

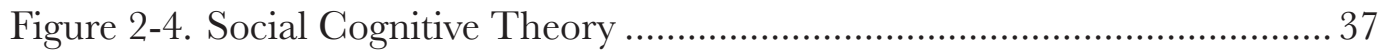

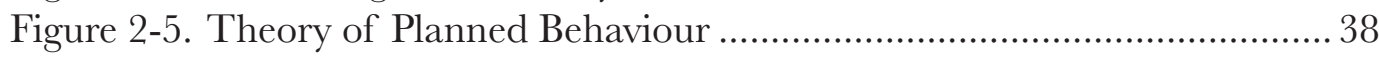

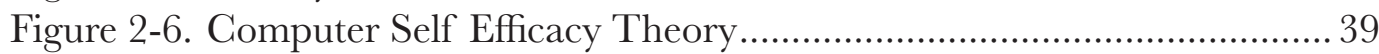

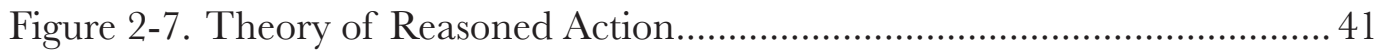

Figure 2-8. Technology Acceptance Model......................................................... 43

Figure 3-1. Proposed internal factors influencing individuals to engage in

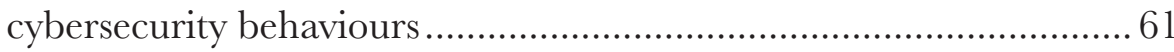

Figure 3-2. Proposed external factors influencing individuals to engage in

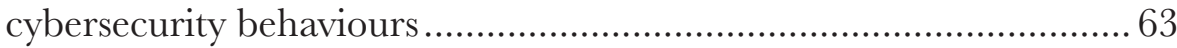

Figure 3-3. Conceptual model for examining home users' reasons for engaging in cybersecurity behaviours

Figure 4-1. Internal factors that influenced home users' cybersecurity intentions

Figure 4-2. Perceived self-efficacy and demonstrated / actual knowledge

Figure 4-3. External factors that influenced respondents' internet security intentions

Figure 5-1. Conceptual model used to explore the factors affecting home users' cybersecurity behaviours

Figure 5-2. Revised model illustrating the factors influencing home users' intentions to engage in cybersecurity behaviours

Figure 5-3. Relationship between components of internal factors relating to home users' internet security behaviours

Figure 5-4. Influence of components of internal factors on awareness .............. 163

Figure 5-5. Influence of components of internal factors on perceived danger ... 165

Figure 5-6. Influence of components of external factors on awareness

Figure 5-7. Influence of components of external factors on internal factors relating to home users' intention to engage in internet security behaviours

Figure 5-8. Influence of components of external factors on perceived susceptibility to and severity of cybersecurity threats

Figure 6-1. Example of how respondents' features are displayed ........................ 189

Figure 6-2. Overview of the key features of the perceived "normal user" as described by respondents

Figure 6-3. Overview of key features of Ostriches ..................................................... 193

Figure 6-4. Overview of the key features of House and Field Mice .......................... 199

Figure 6-5. Comparison between key features of Ostriches and House Mice.............200

Figure 6-6. Overview of the key features of Crowers and Cluckers ..........................202

Figure 6-7. Overview of the key features of Coyotes ...............................................209

Figure 6-8. Comparison between the key features of Coyotes and Cluckers ..............2 210

Figure 6-9. Overview of key features of Dark Horses .............................................213

Figure 6-10. Comparison between the key features of Dark Horses and Crowers.....215 
Figure 6-11. Comparison of the features of a perceived "normal user" and the identified security user types in this study

Figure 6-12. Comparison between the features of a perceived "normal user" as described by respondents and the average respondent based on the data analysis

Figure 6-13. Comparison of user types with lower levels of perceived self-efficacy (Ostriches, House Mice, Dark Horses)

Figure 6-14. Comparison of user types with higher levels of perceived self-efficacy (Crowers, Cluckers, Coyotes)

Figure 6-15. Comparison of user types with lower levels of knowledge (Ostriches, House Mice, Crowers)

Figure 6-16. Comparison of user types with higher levels of knowledge (Cluckers, Coyotes, Dark Horses)

Figure 7-1. Comparison between the features of a "normal user" and the average respondent based on the data analysis

Figure 8-1. Revised model to explain how the identified factors and constructs affect home users' cybersecurity intentions 


\section{List of Abbreviations}

$\begin{array}{ll}\text { BYOD } & \text { Bring Your Own Device } \\ \text { CSET } & \text { Computer Self Efficacy Theory } \\ \text { DDoS } & \text { Distributed Denial of Service } \\ \text { EULA } & \text { End User Licensing Agreement } \\ \text { HBM } & \text { Health Belief Model } \\ \text { IDT } & \text { Innovation Diffusion Theory } \\ \text { PMT } & \text { Protection Motivation Theory } \\ \text { SCT } & \text { Social Cognitive Theory } \\ \text { TPB } & \text { Theory of Planned Behaviour } \\ \text { TRA } & \text { Theory of Reasoned Action } \\ \text { US-CERT } & \text { United States Computer Emergency Response Team }\end{array}$




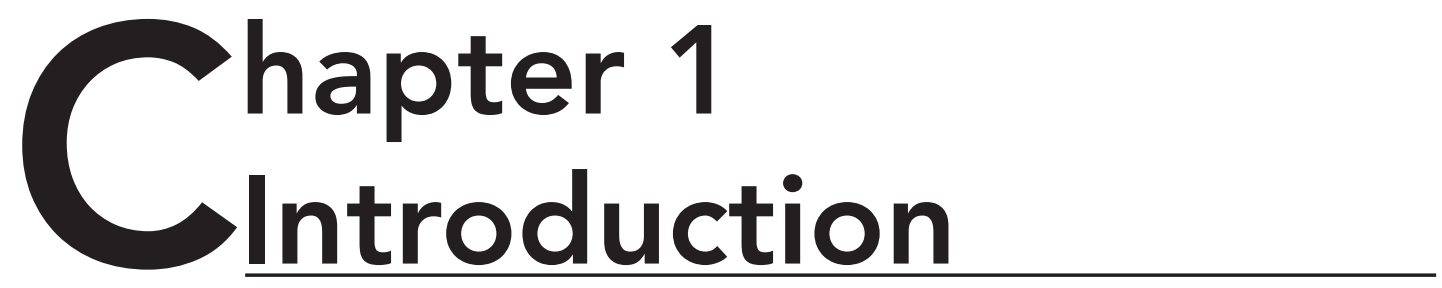

\subsection{Introduction}

Researchers have suggested the Internet is becoming so filled with crime and malicious software that a new Internet is needed because the current technology cannot cope with the ever-increasing threats (Markoff, 2009). In a survey of 8,000 computer users in the United States and Europe, more people said they were afraid of being the victim of cybercrime than they were of being burgled or assaulted (AVG Anti-Virus and Internet Security, 2008; Ferraro \& Parayno, 2008).

In April 2009 the financial cost of one particularly notable worm, Conficker, was estimated to be as high as $\$ 9.1$ billion USD (Cyber Secure Institute, 2009). Because of various cybersecurity threats, in 2008 the US Department of Defense temporarily banned the use of "thumb drives, GDs, flash media cards, and all other removable data storage devices" on computers connected to their intranets (Shachtman, 2008).

In March 2009, a new piece of ransomware ${ }^{1}$ was discovered (Keizer, 2009). One version of this ransomware attempted to get $\$ 50$ from each affected user in order to restore

1 Ransomware is a piece of malware that encrypts files on a user's computer and then promotes another piece of software (typically malicious) to restore the files for a fee, effectively holding the user's files ransom. This not only provides malicious users with the opportunity to install further malware on a user's computer, but also to get their credit card information if they pay the fee to restore their files. 
their files (Keizer, 2009). Although computer security companies have come up with a way to restore the files for free, the danger in this ransomware comes from the fact that it has yet to be caught by antivirus vendors because "it is 'polymorphic' and appears as a different executable file each time" (Wilson, 2009). Ransomware is not new, and Kaspersky Labs discovered a similar piece of ransomware in 2008 (Kaspersky Lab, 2008). Ransomware and other similar software is a growing threat. In 2012 a particular form of ransomware demanded $\$ 200$ within 72 hours or the malicious users claimed that the US Department of Justice would be contacted and that individuals would be subject to fines and imprisonment (Goodin, 2012). In 2013, a less malicious form of ransomware demanded that users fill out a survey in order to unlock their computers (Goodin, 2013).

Without any protective software, the average time it takes for a computer to be infected once connected to the Internet is between 2 and 6 minutes (Internet Storm Center, 2013). However, it is not just new computers that are vulnerable. A report produced by Secunia in December 2008 showed that in a scan of 20,000 computers, less than two percent of those computers were fully patched ${ }^{2}$, and 45 percent of the scanned computers were running more than 11 insecure applications that could be exploited (Jackson Higgins, 2008). In early 2009, a flaw in the Adobe Reader was discovered that would let a malicious PDF file exploit this flaw without any user interaction allowing malicious users to circumvent detection of their malware (Jackson Higgins, 2009).

Although technology and protective software provide some level of protection, technological safeguards alone have not been sufficient to prevent cybersecurity attacks. Malicious users count on being able to circumvent protective technologies, so it is necessary to look to behaviours that are not wholly dependent on technology and security vendors. In order to further support the protective software and increase their overall online security, users can change the way they act online. When protective behaviours are used in combination with technological solutions a user has much more comprehensive security than they would otherwise have using only one or the other. However, in order to convince users of the important role they play in cybersecurity, it is necessary to determine the factors that influence users' intentions to engage in cybersecurity behaviours.

$2 \quad$ Patches refer to software and operating system updates that fix problems, update functionality, or close security vulnerabilities in the system. Failure to ensure that a piece of software is patched can result in vulnerabilities that can be exploited by malicious users. 


\subsection{Background}

Cybersecurity threats include malicious software and the actions of malicious users that can lead to cybersecurity breaches. Cybersecurity threats can affect individuals' computers (for example, making data inaccessible) or the users themselves (for example, by making use of private or sensitive data). It is important to understand the nature of the threats users face online before considering how they can protect themselves from these threats. This section contains an overview of the cybersecurity threat landscape, followed by an overview of some of the safeguards that individuals can use to protect themselves online. This is followed by brief discussion of why there is a need to research the factors that influence users' cybersecurity behaviours.

\subsubsection{Cybersecurity Threat Landscape}

Threats can be transmitted via a number of channels. Email, web pages, network connections, and hardware all pose potential issues. Security companies have categorised the most common types of threats into four main categories (see Panda Security, 2008; Smith, 2004; Sophos, 2008; Symantec Corporation, 2008; Trend Micro, 2008). Contentbased threats, such as phishing and spam are those where the risk is contained in the content of an email. Threats such as viruses, trojan horses, and worms fit into the category of malware-based threats, which arise through malicious software. Web-based threats such as iFrames and script threats can be hidden on legitimate websites. Finally, privacybased threats refer to those that can disclose personal information, either intentionally or unintentionally to unauthorised parties.

Security companies have estimated that between 10 and 60 billion spam ${ }^{3}$ emails are sent daily, equating to between 50 to 80 percent of total daily email volume (Symantec, 2013). The number of phishing ${ }^{4}$ emails tends to be much lower than spam emails, accounting for less than $0.001 \%$ of all emails (Symantec Intelligence, 2013). However, the main danger with these emails is that they are aimed at convincing users to disclose information such

3 Spam refers to unsolicited emails which usually attempt to see something or to offer a disproportionately high benefit to the recipient (Australian Communications and Media Authority, 2007).

4 Phishing uses social engineering techniques to gain sensitive information from the targeted person, often through spoofing. Spoofing is when a malicious user impersonates a legitimate website or person to gain the recipients confidence (United States Computer Emergency Readiness Team, 2001). 
as passwords or credit card details (see Anti-Phishing Group at Indiana University, 2006; Kirda \& Kruegel, 2006). Trusted websites such as PayPal and eBay were the subject of 59 percent of phishing e-mails in 2007 (Sophos, 2008). Because the sites being spoofed are often trusted websites, users are more likely to be susceptible to threats that may have severe consequences for them. The estimated cost of phishing in 2012 was $\$ 1.5$ billion worldwide (BizTech2.com Staff, 2013). Beyond the potential financial consequences, a further risk with content-based threats is that they will link users to malicious software, or malware.

Malware-based threats, such as viruses ${ }^{5}$, trojan horses ${ }^{6}$, and worms ${ }^{7}$ make up the majority of online threats. A report from Panda Security showed that their security scans had revealed that over $30 \%$ of computers worldwide were infected with malware, predominantly trojan horses (Corrons, 2013). The severity of malware can range from being a simple annoyance to data destruction and corruption to privacy breaches that have financial consequences (see, for example Goldsborough, 2007; Helmreich, 2000). However, trojan horses alone are estimated to cost businesses approximately $\$ 114$ billion USD in 2013 and to amount to over 1.5 billion hours and $\$ 22$ billion USD for consumers to recover (Ashford, 2013). More recently, polymorphic threats have become of particular concern because they can evade antivirus software by changing their signature (Cluley, 2012). This makes it imperative that users do not fully depend on antivirus software to prevent threats since it cannot offer complete protection.

Web-based threats use the internet to spread without any additional user actions, such as opening an email attachment or downloading a file (Trend Micro, 2008). Some of the common web-based threats include AJAX and JavaScript vulnerabilities ${ }^{8}$, hacked websites, and the use of iFrames ${ }^{9}$ (Sophos, 2008; Trend Micro, 2008). Another web-based vulnerability, cross-scripting ${ }^{10}$, allows a server to run code when a malicious user enters

$5 \quad$ Viruses are programs that replicate themselves on users' computers similar to how a human virus replicates in a person's body (Australian Communications and Media Authority, 2007).

6 Trojan horses are programs that appear to do one thing, but instead do something else, usually harmful to the computer in some way (Smith, 2004).

$7 \quad$ Worms are pieces of malicious software that travel across computer networks. Unlike viruses and trojan horses, they do not require another program to launch them (Smith, 2004; Staniford, Paxson, \& Weaver, 2002).

8 AJAX and JavaScript Vulnerabilities refer to website tools that can be used to speed up websites, but that are also vulnerable to attacks by malicious users (Trend Micro, 2008).

9 iFrames are invisible hidden elements on webpages that allow malicious users' to compromise legitimate websites (Trend Micro, 2008) 
unexpected data on a site (Trend Micro, 2008). The main danger of web-based threats is that they target legitimate websites that users expect to be trustworthy, such as banks, schools, companies, government agencies, and security forums (Trend Micro, 2008). Because of the nature of these websites, users need to be even more aware that malicious software can be found where they least expect it.

Unlike web-, malware-, and content-based threats, privacy-based threats focus more on the end result rather than the transmission method. Privacy-based threats "relate to the ability of individuals to control information about themselves" (Petersen \& Worona, 2006, p. 16). Information may be disclosed intentionally or unintentionally. Intentional disclosures may be caused by posting personal or identifying information on sites like Facebook. Unintentional disclosures may be the result of malware- web-, or content-based threats. In either case, the information may be used to commit identity and financial fraud, to gain access to other accounts, or disallow access to accounts by the legitimate owner, such as what happened to Wired writer Mat Honan (see Honan, 2012) who lost access to his accounts and had his computer and phone data wiped by malicious users who wanted to gain access to his Twitter handle.

Despite the fact that often users consider themselves to be knowledgeable about safe behaviours, their main weakness lies in themselves. Users may not engage in protective behaviours, even if they are aware of such behaviours, leaving themselves open to risks (Albrechtsen, 2007; Garrison, 2008). At times, they may choose to override the security decisions that a computer or program makes (see Adams \& Sasse, 1999; Aytes \& Connolly, 2004; Culnan, Foxman, \& Ray, 2008). Risks specifically associated with users' failures to act safely in regards to their cybersecurity have been termed "user-related risks" because they depend on the user's behaviour rather than a technological weakness that can be exploited (Aytes \& Connolly, 2004). Therefore, if they do not understand the importance of being secure and understand what secure behaviours actually entail, they may be putting themselves at greater risk.

Although the threats covered above are predominantly technical in nature, they can all be prevented or mitigated through both technical and behavioural safeguards, which should be used in conjunction with each other to provide more comprehensive protection.

\subsubsection{Cybersecurity Safeguards}

Practising computer security has been defined as "apply[ing] a set of recommended practices that would facilitate the achievement of the three common information security 
objectives - confidentiality, integrity, and resource availability" (OECD, 2002 as cited in Ng \& Rahim, 2005, p. 237). Ng and Rahim further indicated that there was "a lack of credible academic sources that agreed on a common set of recommended practices in relation to the security of a home computer system" (p. 237). Although almost a decade has passed since this statement was made, their assertion remains true.

Users can protect their cybersecurity using both technological and behavioural safeguards. Technological safeguards such as firewalls, antivirus software, anti-spyware software and other pieces of protective software can be used to prevent and remedy breaches. Dinev and $\mathrm{Hu}$ (2007) use the term "protective technologies" to describe software "designed to deter, neutralize, disable, or eliminate the negative technologies or their effectiveness, such as anti-virus software, anti-spyware tools, firewalls, and intrusion detection technologies" (p. 387). Protective technologies can be used to prevent cybersecurity breaches. However, these technologies have their own weaknesses. Users are able to disable and override their protective software, which can leave them vulnerable to threats online. While protective software offers a layer of defence against threats, the danger is that it does not offer complete protection and it is reactive, only reacting to threats once they are known (see Perlroth, 2012). There can be delays between when threats are discovered by malicious users and when they are identified by internet security companies (see Perlroth \& Sanger, 2013). During this time, users can be vulnerable even though they are using protective technologies. This suggests that while protective software can be beneficial, on its own, it cannot offer complete protection and therefore for people to increase their level of security, additional steps need to be taken to support the protective technologies.

To support protective technologies, users can undertake protective behaviours. Stanton et al. (2005) identified two beneficial types of behaviour in their taxonomy of security behaviours: aware assurance and basic hygiene. Aware assurance relates to using "technical expertise together with a strong intention to do good" to protect the organisation's systems (p. 126). Unlike aware assurance which requires technical expertise, basic hygiene only requires a "clear intention to preserve and protect the organisation's IT and resources" (p. 126). However, an intention to "preserve and protect" a system does not always translate into the use of protective behaviours if individuals are unaware of the steps that need to be taken. This lack of awareness may be especially present where individuals are not required to undertake security training. Some of the most common behaviours that individuals can engage in to help protect their cybersecurity are: engaging in good password hygiene, using protective software, and ensuring that files are backed up in order to restore the system if something goes wrong.

Secure passwords have long been touted as one of the steps that users need to 
take to protect their security. However, people often do not create secure passwords. A recent breach of IEEE.org showed that approximately $18 \%$ of users were using easy to guess passwords, such as "password" and "123456" (Gallagher, 2012). Despite the call for good passwords, users continue to create weak passwords. Even with good password behaviours, passwords remain a weak form of protection. As computer power increases, the time required to crack passwords using brute force techniques decrease. A 10 character password that was estimated to take 15,000 years to crack in 2008 (Garrison, 2008) could now be cracked in approximately 3 days (Guzik, 2013). Therefore, although good password behaviours can offer some level of protection, these behaviours alone are not enough to protect a person's online security and users increasingly need to understand what additional steps can be taken to help secure their information.

Data backups can be used to help mitigate the loss associated with cybersecurity breaches. However, there are several dangers associated with backups. It is possible for corrupted data to be backed up before the user realises it has become corrupted. Backups also have the potential to become obsolete as programs and file formats change. In addition, if users only backup the files they feel are important, they may miss files they do not realise the importance of until it is too late and the files have been lost. Because of this, it is especially important for users to understand and undertake a comprehensive backup procedure.

\subsubsection{Home Users and Security}

Although individuals may feel that they do not have a lot to risk if their home computers are compromised, such a compromise may have unintended consequences both within the home and outside of the home if it allows data to be stolen or malicious software to be installed. Malicious software can make use of home computers in botnets and to participate in distributed denial of service attacks against businesses (see Furnell et al., 2007). One of the main reasons why home computers are such attractive targets for malicious users is that they do not have protection that is as comprehensive as is on organisational systems. It has been estimated that between 17\% (Eichorn \& Fucanan, 2012) and 24\% (Rains, 2013) of personal computers are not protected. Not using protective software has been estimated to make computers 5.5 times more likely to have viruses (Rains, 2013).

Home users may be less aware of threats than organisational users because they are not required to participate in security training. A lack of awareness and understanding of 
threats has been shown to be an issue for novice internet users in relation to their use of security (Furnell et al., 2008). Talib, Clarke, and Furnell (2010) indicated that there is "little evidence demonstrating whether home users are in fact knowledgeable about information security and indeed practicing it" (p. 197). Although Talib et al. (2008) considered the effect of knowledge on home users' security behaviours, additional factors that influence users' cybersecurity behaviours remain relevant.

In 2005, Ng and Rahim indicated that so far there had been little investigation into home users' security behaviours. Several studies into home users' security behaviours have been undertaken in subsequent years. However, evidence suggests that users are not undertaking the necessary security behaviours as the number of security breaches continues to grow. Therefore, it is necessary to look at what factors encourage and inhibit the use of cybersecurity behaviours.

\subsubsection{Encouraging Cybersecurity Behaviours}

One of the biggest challenges that researchers such as Albrechtsen (2008) and Garrison (2008) have found is that although users seem to know what they should do, there is a gap between what they know and what they do. These researchers have suggested that this gap can be explained by the lack of threat and safeguard awareness, a lack of understanding about how to prevent cybersecurity threats, a lack of time to maintain cybersecurity behaviours, the cost of the safeguards, and security overload.

Users need to be aware of both the potential cybersecurity threats as well as the safeguards and the associated benefits of using the safeguards. If users are not aware of the threats and safeguards, it is more difficult for them to access information that will help increase their awareness of safer computing behaviours (see Albrechtsen, 2007). However, security experts often keep information about threats and vulnerabilities from being exposed until after there is a fix in place in the hope that this will decrease the exploitation of the vulnerabilities (see Adams \& Sasse, 1999; Naraine, 2008). Although users are expected to increase their awareness of threats and safeguards, they are often unable to do so until after they have already been vulnerable to the threat. Symantec reported that the average time before a zero day bug ${ }^{11}$ is detected is 312 days (Perlroth \& Sanger, 2013). This creates a tension between what the security companies and computer experts expect of users and

11 Zero Day Bugs are threats "...that once discovered, "zero days" exist for the user of the computer system to fix them before hackers can take advantage of the vulnerability. A "zero-day exploit" occurs when hackers or governments strike by using the flaw before anyone else knows it exists" 
what the users are able to do in regard to increasing their awareness since there is often an extended period of time when users are vulnerable.

Even if users know that there is a need to protect themselves from threats, they may not understand how to protect themselves or how to configure the protective software in the most appropriate ways (Albrechtsen, 2007). Researchers have found that users often do not understand how to protect their computers, resulting in the failure to take the necessary protective steps (see AOL/NCSA, 2005; Furnell, Bryant, \& Phippen, 2007; LaRose, Rifon, $\&$ Wirth, 2007). In addition, users may not understand that it is their responsibility to protect their security. In the past, researchers have found that users often consider it the responsibility of their ISP to protect them from cybersecurity threats (see Furnell et al., 2007; LaRose et al., 2007).

Organisational research has shown that users are less likely to engage in safe practices if they perceive that their time is better dedicated to other matters (Albrechtsen, 2007). Although these findings were from an organisational context, similar issues can be present for home users. Home users not only need to take the time to engage in safer practices themselves, but may also be responsible for ensuring that other users of the computer are aware of what they need to do to stay safe.

Even when users are aware of the safeguards they can use, they may find protective software to be too costly (Furnell et al., 2007). Although free protective software is available, this requires users to be aware of the existence of free protective software and know where to get it. In addition to the costs associated with protective software, there are also additional costs such as storage media associated with conducting backups. If users do not see the value in taking steps to protect their computers and data, it may be even more difficult to justify the costs associated with protective software and other protective steps.

Security overload refers to the high number of passwords that users need to remember and maintain regularly (Albrechtsen, 2007). Security overload may actually lead to poorer security behaviours because it can cause users to write down passwords which increases the possibility of someone gaining access to the passwords and misusing them. It can also lead to the reuse of passwords across sites, which can increase the likelihood of a person gaining access to important information by targeting less important sites. These behaviours increase the risk of a data breach by either making the password more readily available or by allowing malicious users to gain access indirectly. 


\subsection{Research Problem, Questions, and Objectives}

The existence of threats, such as those identified in section 1.2.1, creates the context for the research problem. Based on these threats, this section identifies the research problem, research questions, and research objectives for this study.

\subsubsection{Research Problem}

The number of threats that exist in the cybersecurity context is growing and both threats and attackers are becoming more sophisticated. Because of this, it is becoming increasingly difficult for technological solutions to keep up with the threats. Even when security software is utilised, it is still possible for a computer to become infected. In a survey of approximately 1.5 million computers by Panda Security, 37\% were using fully up-to-date software, but $25 \%$ of those computers still had at least one infection on them, equating to 127,000 infected machines, not including computers that were not fully protected (Grossman, 2007). Security software cannot detect all possible threats, with some of the best security software detecting only 64 out of 300 potential vulnerabilities (Markoff, 2009). Because of the limitations of solely technological protection, users need to be aware of what they can do to protect themselves from cybersecurity threats. While researchers have suggested that awareness can contribute to use of protective behaviours (Aytes \& Connolly, 2004) and software (Dinev \& Hu, 2007), there has been little research into what factors contribute to this awareness and how influential it is. Therefore, there is a need to further investigate the role that awareness has in influencing users' cybersecurity behaviours.

Although users need to be aware of these threats and how to avoid them, researchers and practitioners have often stated users are the weakest link in the security problem (see Lineberry, 2007; Sasse, Brostoff, \& Weirich, 2004). Users are able to override the decisions computers make. Even if anti-virus software tells a user something may be infected, a user can choose to ignore the warning and proceed regardless. The ability for the user to override automated decisions is necessary in case the security program incorrectly identifies a threat. However, the same ability to override warnings makes it easier for users to ignore these suggestions made by their computers. Users can also engage in behaviours that may endanger their cybersecurity. These behaviours may be done out of ignorance, laziness, even a belief that the user is invulnerable to threats (see Rhee, Ryu, \& Kim, 2005). Furthermore, cybersecurity threats frequently rely on users' weaknesses by using social engineering, which is a "nontechnical hack... [that uses] trickery, persuasion, impersonation, 
emotional manipulation, and abuse of trust to gain information or computer system access through the human interface" (Thompson, 2006, p. 222). This suggests that there is a need to consider security from the user perspective. In particular, there is a need to investigate the factors that influence home users' behaviours because of their differences from organisational users and a shortage of research into the factors that influence home users' behaviours.

A lack of knowledge, skill, motivation, or finances can also contribute to users' failure to protect themselves. Even more worrying is that although users indicate that they know what they should be doing to protect themselves online, they still do not take these steps (see Aytes \& Connolly, 2004; Furnell et al., 2007; Furnell, Tsaganidi, \& Phippen, 2008). Although several reasons have been identified for why users may not engage in safer practices, it is still unclear what factors influence a user's decision to engage in safe or unsafe computing practices. The presence of reasons for why users do not protect themselves combined with the gap between what people know and what people do suggests that there is a need to look at the factors that influence users' intentions to engage in safer behaviours.

The most commonly researched factors have been: perceived severity, perceived susceptibility, self-efficacy, attitude, and the subjective norm. Although these factors have been identified as being particularly important to influencing users' cybersecurity behaviours, there has been little agreement about how they do so, which factors play the greatest role, and how these factors interact and influence each other. In addition, it is necessary to look at which factors are most influential in the home user context, rather than just the organisational context.

\subsubsection{Research Questions}

Based on the preponderance of cybersecurity threats, the need to help secure home users from these security threats, and the lack of knowledge about what factors influence users with respect to their cybersecurity practices, this research seeks to answer the following research questions:

1. What are the factors influencing home users' cybersecurity behaviours?

2. How do these factors influence home users' cybersecurity behaviours?

By looking at what factors influence users' intention to use security behaviours, there are implications for future research and practice. For future research, this provides another step toward understanding why people continue to act in an unsafe manner. Furthermore, it focuses on the individual rather than technology and the associated adoption factors. 
More practically, by understanding the factors that influence users' behaviours, it may be possible to identify a starting point for how security messages can better target individuals and be considered as more relevant. This opens up further possibilities of looking at how security messages can be marketed.

\subsubsection{Research Objectives}

This research has three objectives. These objectives are to:

1. Identify the factors affecting home user cybersecurity practices.

2. Develop a framework to explain how these factors influence home users cybersecurity behaviours.

3. Determine how the factors relating to awareness of threats and safeguards affect home users' cybersecurity practices.

\subsection{Theoretical Justification}

Much of the research on cybersecurity has taken a technology-based approach, focusing on either the creation of new technologies or why people adopt protective technologies. There is still a need to examine the behaviours in which users can engage to protect themselves online, and to determine what influences users to engage in these behaviours. The need for a behavioural perspective is further justified in section 2.3.3.

Although there is a growing body of research on cybersecurity, the topic is still broad and undefined with a number of gaps in the research. There remains a lack of research examining home user cybersecurity behaviours. The most prevalent type of cybersecurity research on behaviours appears to be focused on cybersecurity behaviours within the organisation. Thus there is a need to examine cybersecurity from a home user perspective.

Finally, although cybersecurity appears to be a technology-centric issue, user behaviours also play a strong role. Users are able to override the decisions technology makes, therefore, making user behaviours a central aspect of cybersecurity. Wholly technological theories cannot fully explain the cybersecurity dilemma. Because of this, researchers have begun to utilise behavioural theories to explore what influences users' behaviours in the cybersecurity domain (see for example Harrington, Anderson, \& Agarwal, 2006; LaRose, Rifon, \& Enbody, 2008; Ng \& Rahim, 2005). However, there is still a lack of understanding about why users to engage in their chosen cybersecurity behaviours. Therefore, further research into the factors that influence home users' cybersecurity behaviours will contribute 
to the body of research in this area.

\subsection{Practical Justification}

The number of people online has increased to over two billion (Internet World Stats, 2011). As the number of people online increases, so does the number of people and computers that are potential targets. The increased number of targets also creates an incentive for cybercriminals.

As the number of Internet users increases, so does the amount of sensitive data transmitted online. Within New Zealand, approximately two-thirds of Internet users have used Internet banking (Neilsen Company, 2008). In 2012, approximately \$186.2 billion USD were spent in the United States on e-commerce transactions (Protalinski, 2013). In New Zealand, online spending totalled approximately $\$ 3.2$ billion NZD with the average individual spending \$1700 NZD in 2012 (PwC \& Frost \& Sullivan, 2012). E-commerce transactions require sensitive information to be transmitted. Any time data is transmitted online it is possible for it to be intercepted by a malicious party. Online shopping is expected to continue to rise, presenting more opportunities for users to have confidential information accessed.

The decreased cost and increased capacity of portable mass storage devices combined with an increase in users working both at home and at work has made it possible for users to carry large amounts of data between locations. Increased data portability creates the potential for users to bring hazards acquired in the home into the workplace, essentially overriding security that may be in place within the organisation. The costs of malware within organisations has risen from approximately $\$ 13$ billion USD in 2006 (Computer Economics, 2007) to $\$ 114$ billion USD in 2012 (Ashford, 2013).

\subsection{Overview of Methodology}

This research utilises a qualitative, interpretivist approach. Currently, there has been little qualitative research on home users' cybersecurity behaviours. Although some quantitative studies on cybersecurity have been carried out, the focus of these studies has been on predetermined factors created by researchers. The qualitative perspective used in this research has allowed participants to "use their own words and images and draw on their own concepts and experiences" (Orlikowski \& Baroudi, 1991, p. 15), which has allowed more concepts to be examined, including those which had been identified in 
previous research.

In the preliminary research for this study, eight studies were identified as being relevant to examining home user cybersecurity behaviours. In these studies, quantitative research was the predominant approach (see Aytes \& Connolly, 2004; Dinev \& Hu, 2007; Furnell et al., 2007; Harrington et al., 2006; LaRose \& Rifon, 2007; LaRose et al., 2008; LaRose, Rifton, Liu, \& Lee, 2005; Ng \& Rahim, 2005). While these studies have been able to find out user perspectives on topics such as email and antivirus behaviours, a potential weakness is they did not give the respondents a chance to discover factors that influence their security behaviours that were not already identified by the researchers. This research seeks to identify the primary factors that influence home users' cybersecurity related decisions.

In this research, three focus groups and 20 semi-structured interviews were conducted in order to identify the factors related to cybersecurity behaviours. Focus groups provided information about users' shared understandings related to the topic explored (see Basch, 1987). In order to explore topics raised during the focus groups in greater depth, individual interviews were conducted. The sample selection process is described in further detail in Chapter 3.

\subsection{Delimitations}

The purpose of this study was to explore home users' internet security behaviours. Therefore, the target population needed to be one that could be assumed to have control over their security behaviours. The participants in this study were limited to adult Internet users because it was assumed they would have more control over the decision to engage in cybersecurity behaviours, including the use of protective technologies. Adults were more likely to be in a position to make decisions for a household about purchasing protective technologies. In addition, adults were more likely to be in a position to develop and enforce household rules about the use of technology.

This research focused on providing a deeper exploration of the factors that influenced users' behaviours and proposing relationships between these factors. Although the model could be tested quantitatively, for the purposes of this study the qualitative results provide the foundation for future quantitative research. In addition, this study was focused on users' ongoing behaviours. Therefore, using an experimental approach was not appropriate because it would focus more on single points in time rather than ongoing influences. 


\subsection{Assumptions}

Several assumptions were made in planning this research. First, it was assumed that some people acted in an unsafe manner online. This assumption was supported by the continued prevalence of internet security breaches that occur every year. It was also assumed that individuals would be able to identify their reasons for engaging or not engaging in safe behaviours online. This was underscored by the belief that even if individuals did not always act in a rational manner, they would understand why they took the actions that they did. Because of the potential for irrational decision-making related to cybersecurity, several theories which address this are discussed in the literature review. It was assumed that users who did not do anything in relation to their cybersecurity behaviours would be able to identify why they did not take protective steps. Finally, it was assumed that respondents would accurately report their cybersecurity behaviours and the steps that they took to protect themselves online.

\subsection{Definitions}

Cybersecurity (further defined in section 2.2.2) refers to the protection of data from unauthorised use and from unwanted destruction by Internet threats (including, but not limited to spam, phishing, malware, and other web based threats) (United States Computer Emergency Readiness Team, 2008).

Cybersecurity threats (also referred to as threats) refer to malicious software and the actions of malicious users which can lead to cybersecurity breaches (Dinev \& Hu, 2007).

Cybersecurity breaches (also referred to as breaches) refer to situations where cybersecurity threats affect computers, leading to data, personal, or other consequences which require steps to be taken to remedy the breach (Bagchi \& Udo, 2003). Data consequences can include disclosure, corruption, and other access issues, while personal consequences can include financial, emotional, and other consequences unrelated to the actual data.

Malicious users refer to the individuals who write, propagate, and are otherwise responsible for cybersecurity threats. Despite the term "hackers" often being used to refer to malicious users, it should be noted that hackers do not always act maliciously (Young, 
Zhang, \& Prybutok, 2007). Therefore, the term malicious users is used in this study.

Protective behaviours (also called cybersecurity behaviours) refer to the steps that individuals take to protect their security online (Ng \& Rahim, 2005). This can involve both behavioural safeguards (such as those discussed in section 1.2.2) and the use of protective software.

Protective software refers to software, such as antivirus software, firewalls, and antispyware software, that is used to identify and prevent cybersecurity breaches (Dinev \& $\mathrm{Hu}, 2007)$.

Further relevant definitions can be found in Appendix 1.

\subsection{Outline of the Thesis}

The first chapter of the thesis has identified the main cybersecurity problems for users and the ways they can address these problems. Based on these threats and the lack of research on home users' internet security behaviours and what influences them, the research problem was identified. Next, an overview of the research justification was presented along with the delimitations, limitations, and assumptions associated with this research. Finally, the key terms were defined.

Chapter 2 provides a review of the relevant cybersecurity literature. The synthesis of the literature led to the identification of four approaches that cybersecurity research has used. The shortage of research exploring home users' cybersecurity behaviours led to the selection of home users' behavioural safeguards as the broad area for this research. The main factors identified as being relevant to individuals' cybersecurity behaviours are identified and examined.

Chapter 3 provides a discussion of the methodology used in conducting this research. Based on the factors and relevant models explored in Chapter 2, a conceptual model used to guide the research is developed, presented, and explained. Using the conceptual model as the foundation, the data collection and analysis procedures are justified and discussed. An interpretive approach was taken when analysing the qualitative data collected from focus groups and semi-structured interviews.

The findings from the data are presented in Chapter 4. Six main factors were identified as being relevant to influencing respondents' cybersecurity behaviours: awareness, 
internal factors, external factors, perceived danger, perceived barriers and enablers, and cybersecurity intentions. Each of these factors is discussed and the relevant sub-factors within each of the factors are identified.

Based on the data, a revised model is presented in Chapter 5. Within this chapter, the relevant relationships between the factors and sub-factors are identified and justified based on the data.

From the revised model, several factors emerged as being particularly relevant to influencing respondents' behaviours. Based on these factors, five types of respondents are identified and explained in Chapter 6. These factors are used to further illustrate how particular combinations of factors explored in Chapters 4 and 5 were common to certain types of security behaviours.

In Chapter 7, the main findings are discussed in light of the literature. In addition, the development and use of user types to further illustrate the model are discussed within the context of current literature on user types.

Finally, in Chapter 8, the conclusions on the research questions are presented along with a discussion of the implications of this research on theory and practice. Next, areas for future research are identified. Finally, the limitations of this research are identified and discussed. 


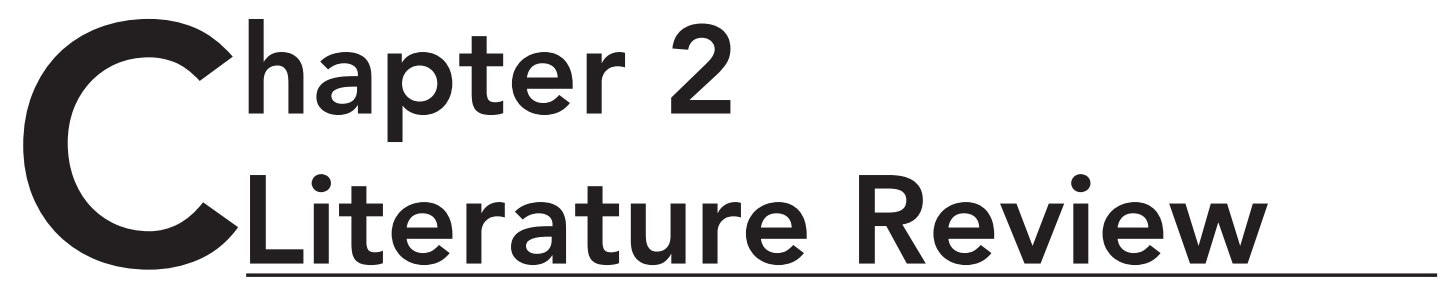

\subsection{Introduction}

This chapter presents a review of the literature used to gain a greater understanding of the research problem. The desire for security is the starting point for understanding internet security. An understanding of general security can help to explain why we act the way we do when faced with security threats. In this chapter, the relevant literature is explored based on four main categories of research that were identified during the literature search: the type of actor involved in the behaviours (e.g. home or organisational users); the goal of the behaviours (e.g. security or privacy); the approach taken to achieving the goal (e.g. social / behavioural approaches or technology approaches); and the duration of the steps taken (e.g. one-time adoption or ongoing behaviours). Distinguishing the literature into these categories created a clearer understanding of which areas had been researched in the past and which areas needed further research. By looking at how past research has explored these aspects of security, a gap in the literature relating to understanding what influences home users' internet security behaviours is identified and justified. As a starting point for addressing this gap, a review of the factors identified in past research relating to security behaviours is presented. 


\subsection{Security \& Cybersecurity}

An understanding of security is the starting point for understanding cybersecurity. Although security often refers to the protection of an individual's personal safety, it can also be applied within the cybersecurity context and refer both to individuals' virtual safety in relation to their computers and data and to their perception and feelings of safety.

\subsubsection{Security}

The need for safety and security has been identified as one of a person's basic needs. Maslow (1970) identified five basic needs that motivate people's behaviours: psychological needs, safety needs, social needs, esteem needs, and self actualisation needs. Once lower level needs, such as physiological needs, are satisfied, people will move on to trying to satisfy higher levels needs, such as safety. In particular though, the need for safety is especially prominent in emergency situations where a person's stability and perception of safety is disrupted (Maslow, 1970). These same needs may exist within the Internet context as well, especially when individuals' perceptions that they are safe online are disrupted by the consequences of an internet security breach.

The very basis of a cybersecurity breach is a disruption to the online expectations of individuals. The expectation is that the data, files, and information that a person stores on their personal computer will be accessible when needed, free from alteration, and can only be accessed by the appropriate people. However, a cybersecurity breach disrupts this normal routine by causing data to be inaccessible or by causing other unexpected consequences which lead to a feeling that a person's safety has been breached. Although the traditional expectation has been that the criminals from whom we need to protect ourselves are only in the physical world, the use of technology no longer requires them to be physically present to commit a crime. Regardless of whether the security breach takes place online or offline, the person's perception of safety is being compromised unexpectedly, likening an internet security breach to an emergency situation. This suggests that internet security can be likened to a version of personal security. However, researchers have not agreed on a definition for cybersecurity. As a result, it is first necessary to further define what is meant by cybersecurity. 


\subsubsection{Cybersecurity}

There is no agreed upon definition of cybersecurity, primarily because there are so many aspects which can be examined (Wilbanks, 2007). Researchers and practitioners have used the terms cybersecurity, internet security, computer security, and information security in exploring how people and organisations protect their interests online. Bishop (2003) suggests that computer security, network security, and cybersecurity are analogous. While von Solms and van Niekerk (2013) suggest that although cybersecurity and information security are often considered interchangeable, they are different. Furthermore, they suggest that while information security focuses on ensuring the confidentiality, availability, and integrity of data, cybersecurity goes further and also considers the actions of the individual (p. 98). Following on from this, it can be seen that cybersecurity research has generally covered three broad areas:

- network and infrastructure protection,

- system protection, and

- personal system / data protection.

These areas of cybersecurity differ in scope, with network and infrastructure protection typically considering the national level, system protection focusing on the physical security of systems, and personal system and data protection focusing on the individual level. Each of these areas is an important part of cybersecurity as there is the potential for wider consequences when an individual's computer suffers from a cybersecurity breach.

At the broadest level, cybersecurity deals with network and infrastructure protection. This involves protecting the networks and physical infrastructure that allow computers to connect to each other, as well as allowing governments, businesses, individuals, and others to communicate (Bishop, 2003; U.S. Cyber Security Information Act, 2000). When considering cybersecurity, many governments focus on the network and infrastructure level (for example, see Center for Strategic and International Studies, 2008; United States Department of Homeland Security, 2003).

The next level of cybersecurity involves system protection. System protection is primarily concerned with protecting the data and actual information system from physical threats and software-based threats (see Berkowitz \& Hahn, 2003; Chou, 2007; Wilbanks, 2007; ZDNet, 2008). At this level, the primary focus is on protecting the integrity of information systems in businesses and organisations, rather than home systems and 
individual users.

Finally, cybersecurity can be examined from the personal cybersecurity perspective. Personal cybersecurity involves engaging in safe practices as well as protecting personal data (see Petersen \& Worona, 2006; United States Department of Homeland Security, 2003; Wilbanks, 2007). The United States Computer Emergency Readiness Team (USCERT) states that cybersecurity involves "protecting [personal] information by preventing, detecting, and responding to attacks" (McDowell \& Householder, 2004).

For the purposes of the literature review the definition of cybersecurity is based on the definition by the US-CERT and is defined as the protection of personal data from unauthorised use and from unwanted destruction by Internet threats (including, but not limited to spam, phishing, malware, and other web based threats) (see United States Computer Emergency Readiness Team, 2008). Personal cybersecurity is important for several reasons. Files and other data are frequently moved between personal and work computers which allows for weaknesses in the home system to be exploited and potentially bypass stricter workplace standards (see Culnan et al., 2008; Shachtman, 2008). Second, malicious users may exploit personal computers as a pathway to accomplishing other tasks. These malicious users can use the exploited systems to deliver junk emails, disrupt Internet services, and distribute malicious software. Finally, home users are particularly at risk for crime utilising the Internet, such as financial fraud and identity theft, and therefore need to be aware of how to avoid these threats (Crafty, 2009). Approximately one out of every five computers in the US was infected with at least one piece of malware in 2012 (Wilson, 2013).

Some authors do not consider the personal form of cybersecurity to be a true form of cybersecurity because it focuses on the behaviours of individuals rather than the protection of organisational systems and infrastructure (Chou, 2007). However, this type of cybersecurity is as important as the protection of data, systems, and infrastructure. Individuals are involved in the creation, maintenance, and every day decisions that can impact cybersecurity at the network and system levels (United States Department of Homeland Security, 2003). In addition, malicious users can take advantage of users' poor cybersecurity behaviours, allowing the computers to contribute to attacks on governments and businesses (Culnan et al., 2008). Because of the potential for damage both to home users' computers and other computers, personal cybersecurity behaviours are of great importance. 


\subsection{Personal Cybersecurity}

One of the difficulties with cybersecurity research is its broad nature. Even when approaching cybersecurity from a personal cybersecurity perspective, different approaches can be taken. The examination and synthesis of the literature led to the identification of four broad components of cybersecurity research: the actor, the objective, the method, and the duration (see Table 2-1, p. 23). Categorising the literature by the components of the research (see Appendix 2, p. 265) provided a way to see what the predominant approaches have been and were a starting point for identifying the gap in the literature. The actor refers to the person or persons undertaking the protective behaviours. In relation to cybersecurity, the actor is predominantly the home or organisational user. The objective of the actor in cybersecurity is most often related to either protecting a user's privacy or security. While privacy and security are often discussed separately, security is a broader concept that can encompass some of the issues associated with privacy in regards to access and integrity. The method for achieving the objective usually takes either a technology-

Table 2-1. Definitions and descriptions relating to components of cybersecurity research

\begin{tabular}{|l|l|l|}
\hline Approach & \multicolumn{1}{|c|}{ Perspective } & \multicolumn{1}{c|}{ Definition / Description } \\
\hline Actor & Home User & $\begin{array}{l}\text { "Individuals conducting personal (including school-related) } \\
\text { activities on a computer that is not owned or controlled } \\
\text { by an organisation... [these users] are not threatened by } \\
\text { the possibility of losing a job as a result of behaving in an } \\
\text { unsecure fashion online" (Harrington et al., 2006, p. 1544). }\end{array}$ \\
\cline { 2 - 4 } & $\begin{array}{l}\text { Organisational } \\
\text { User }\end{array}$ & $\begin{array}{l}\text { Individuals using a computer owned or controlled by an } \\
\text { organisation primarily for the purposes of work. }\end{array}$ \\
\cline { 2 - 4 } Objective & Security & $\begin{array}{l}\text { "Security is principally concerned with the management of } \\
\text { risk" (Petersen \& Worona, 2006, p. 16). }\end{array}$ \\
\cline { 2 - 4 } Method & Socially-Based & $\begin{array}{l}\text { "Privacy relates to the ability of individuals to control } \\
\text { information about themselves" (Petersen \& Worona, 2006, p. } \\
\text { 16). }\end{array}$ \\
\cline { 2 - 4 } & $\begin{array}{l}\text { Considers what behaviours users can undertake to protect } \\
\text { themselves from cybersecurity breaches and what factors } \\
\text { influence these behaviours. }\end{array}$ \\
\cline { 2 - 4 } Dechnology- & $\begin{array}{l}\text { Uses hardware and software solutions in order to prevent } \\
\text { cybersecurity breaches. }\end{array}$ \\
\cline { 2 - 4 } & One-Time & $\begin{array}{l}\text { Focuses on individuals' ongoing practices that users choose } \\
\text { to engage in on a regular basis (for example, the decision to } \\
\text { always check downloads for viruses). }\end{array}$ \\
\hline \multirow{2}{*}{ Ongoing } & $\begin{array}{l}\text { Implies a one time decision rather than on ongoing } \\
\text { behavioural change (for example, the decision to install } \\
\text { antivirus software) }\end{array}$ \\
\hline
\end{tabular}


based approach or a socially-based approach. The technology-based approach focuses on developing new technologies or products to achieve the objective. In contrast, the sociallybased approach focuses on the actor and changes that can be undertaken with relation to their behaviours to achieve the objective. Finally, the method's duration can also be included in terms of whether the method is associated with a one-time adoption or an ongoing behavioural change.

Not all research has covered all four components (see Appendix 2, p. 265). However, even research that did not cover all four components often covered one or two components. Typically the literature followed a path through these components, starting with the actor, moving on to the objective, then to the method and duration (see Figure 2-1). The technology-based approach to research has been the predominant approach in the past literature. However, in recent years the focus has begun to change to a more social / behavioural approach. Although cybersecurity is inherently technology related because of the need to find ways to identify and prevent threats, the people who use it play a key role. Individuals can choose to ignore or override technical protections. There is no guarantee that the presence of technological safeguards or a greater understanding of threats will help prevent breaches if individuals are still involved in the security process. Therefore, there is a need to determine what makes users act safely or unsafely, both inside and outside of the organisation.

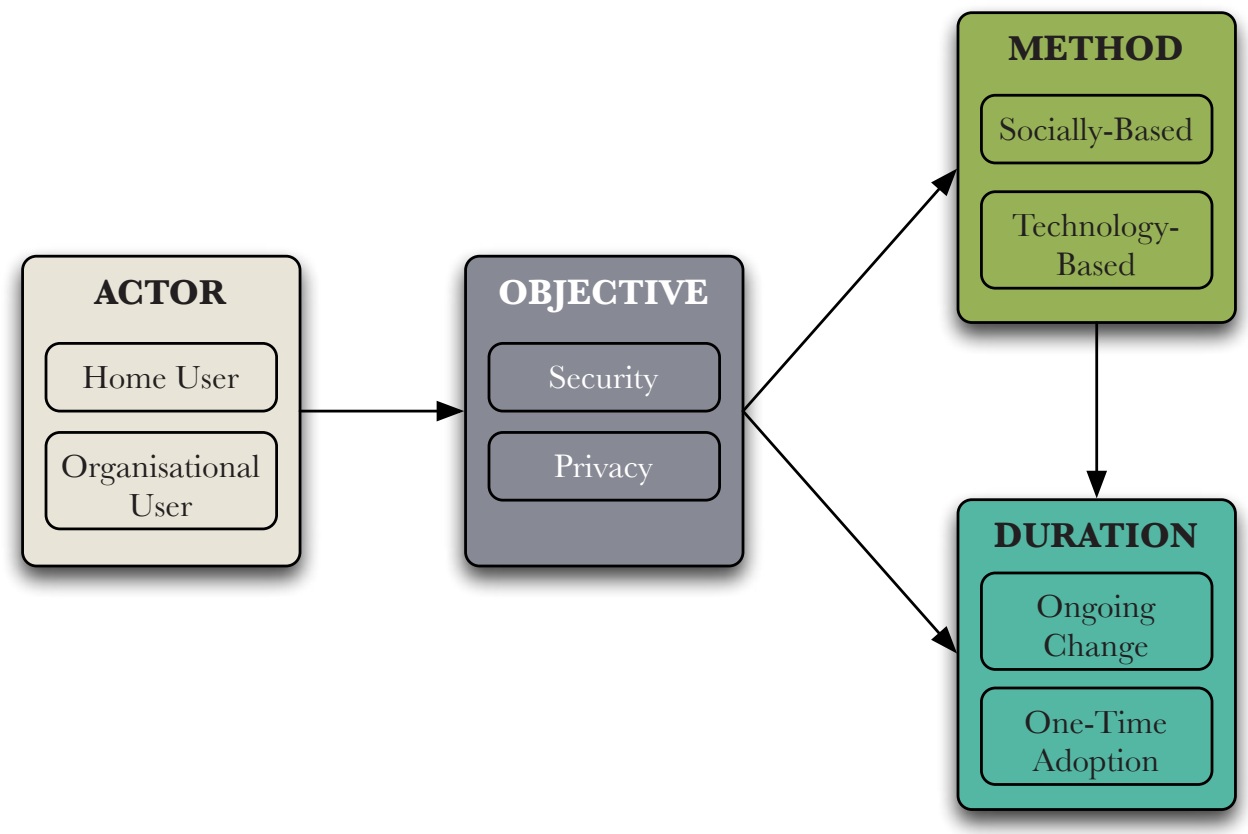

Figure 2-1. Detailed analysis of the components of cybersecurity research 


\subsubsection{Home User}

The actors in cybersecurity are usually either organisations and their users or home users. Harrington et al. (2006) describe home users as including "individuals conducting personal (including school-related) activities on a computer that is not owned or controlled by an organisation...[Home users] are not threatened by the possibility of losing a job as a result of behaving in an unsecure fashion online" (p. 1544). In contrast, organisational users are those users who use a computer either owned or controlled by an organisation that is used primarily, though not necessarily entirely, for the purpose of work. A person who works from home but whose use of the computer at home is primary for matters related to their employment is not, for the purposes of this study, to be considered a home user. A majority of the research to date has focused on the organisational user, leaving a gap in knowledge on what influences home users' behaviours.

Researchers have often indicated that users are the weakest link in the cybersecurity chain, regardless of whether they are home or organisational users (for example, see Adams \& Sasse, 1999; Dinev \& Hu, 2007; Lineberry, 2007; Sasse et al., 2004). Much of the research on security focuses on users at the organisational level. However, researching the home user is especially important as the number of people using the internet at home continues to increase. In 2013, home computer users were expected to spend 1.5 billion hours and $\$ 22$ billion dollars to recover from malware found in pirated software (Ashford, 2013). There is little agreement about the exact number of computers infected worldwide. However, security companies have estimated that between 5\% (Kaspersky, 2013) and 33\% (Panda Security, 2013) of all computers worldwide are infected with at least one type of malware. Malware on unsecured computers can be used to launch attacks on businesses and networks, effectively shutting them down ${ }^{1}$, further increasing the need for good cybersecurity at the network, infrastructure, and organisational levels. In addition, more users online means more potential targets for malicious users to launch these attacks from. Finally, as organisations increasingly allow for "bring your own devices" (BYOD) in their policies and allow for remote working, there is the potential for threats that exist on the home system to be moved into the organisation, effectively bypassing some of the organisation's security measures (Culnan et al., 2008). All of this points to the need for further research on how home users can be encouraged to protect their systems better.

When compared to organisational users, home users are often at a disadvantage

1 In 2013, a botnet that infected millions of computers was shut down in a joint effort between Microsoft and the FBI (Dotinga, 2013, Schwartz, 2013b). This botnet was primarily aimed at collecting banking information from individuals. However, in the court proceedings against the creators of the malware, Microsoft noted that some versions of the software had been used in DDoS attacks against organisations. 
when it comes to security. Home users do not have the same budget that organisations have in regard to managing security. Although free protective software is available, users still consider the cost of the software to be particularly prohibitive (see Furnell et al., 2007). In addition, home users do not have the same level of training or available expertise about security and the steps that can be taken to protect a computer. Because of this, another barrier to engaging in cybersecurity barriers is the lack of requisite knowledge about how to protect the computer (Furnell et al., 2007). Despite efforts by organisations to get employees to follow safe computing practices at work, these practices are not necessarily followed at home, which can increase the potential for threats being brought into the organisation via users' home computers or files (Culnan et al., 2008).

\subsubsection{Security Objective}

Cybersecurity literature has primarily focused on either protecting an individual's security or privacy. Privacy can be explained as "the ability of individuals to control information about themselves" (Petersen \& Worona, 2006, p. 16). However, in order to control that information, security steps need to be taken. Security can be broadly defined as "the management of risk" (Petersen \& Worona, 2006, p. 16). Therefore, although privacy and security are defined differently, the protection of privacy may be encompassed as part of security.

Further supporting the close ties between security and privacy, the US-CERT defines cybersecurity as "protecting [personal] information by preventing, detecting, and responding to attacks" (McDowell \& Householder, 2004). Within this definition, there are aspects of both privacy (e.g. protecting personal information) and security (e.g. protecting that information from breaches through the use of security measures). Often, privacy is concerned with ensuring the information is not disclosed to an unauthorised third party. However, there is no need for a user's information to leave the computer or even to be viewed for there to have been a cybersecurity breach. Threats such as ransomware can prevent an individual from accessing private data without it ever being disclosed. Therefore, there is a need to go beyond just looking at privacy and instead looking at how security can further protect an individual's privacy.

\subsubsection{Behavioural Safeguards}

Technical approaches to cybersecurity often focus on developing, adopting, or using hardware and software solutions to prevent cybersecurity breaches. The behavioural 
approach instead focuses on the actions taken by users, including the use of protective software, and can also include steps taken to avoid or diminish the danger of cybersecurity threats. Social or behavioural based security research often focuses on nontechnical solutions, such as examining what influences people to engage in protective behaviours. The field of understanding user security behaviours and their antecedents has only begun to gain traction in the last 10 years (see Harrington et al., 2006). The most frequently researched topics in the behavioural context examined what people do to protect their security, what influences these behaviours, and the effectiveness of security and privacy messages.

Researchers have found that although many users consider themselves to be knowledgeable about the potential threats, their behaviours do not always match what they know (Aytes \& Connolly, 2004; Furnell et al., 2007). News about threats such as viruses, phishing, and botnets may make users more aware of the threats that they should be concerned about. However, these news stories often have the goal of simply informing people about the threats, rather than educating them about ways they can safeguard themselves against them. While awareness campaigns can be used to educate users, they are often not as effective as other methods because they do not present lasting messages about how to behave securely (Albrechtsen, 2007). Despite research indicating that there is a gap between what people know and what they do, there remains a need to investigate what factors are responsible for the presence of this gap.

Intentions are considered to be accurate predictors of future behaviours (Ajzen, 1991). Because of this, security researchers have begun to examine the factors that lead to the development of intentions to practice security. Many different factors have been identified, but to date there has been little agreement on which factors are most influential in the cybersecurity context (see Table 2-2), or how these factors

Table 2-2. Factors influencing

cybersecurity and online privacy intentions

\begin{tabular}{|l|l|}
\hline Influential factors & \multicolumn{1}{|c|}{ Author(s) } \\
\hline Attitude & Ng \& Rahim (2005) \\
\hline $\begin{array}{l}\text { Descriptive norm / } \\
\text { optimistic bias }\end{array}$ & $\begin{array}{l}\text { Harrington et al. (2006) } \\
\text { Rhee et al. (2005) }\end{array}$ \\
\hline Fear & LaRose et al. (2008) \\
\hline Mass media influence & Ng \& Rahim (2005) \\
\hline $\begin{array}{l}\text { Peer \& family } \\
\text { influence }\end{array}$ & Ng \& Rahim (2005) \\
\hline $\begin{array}{l}\text { Perceived benefits of } \\
\text { practicing security }\end{array}$ & $\begin{array}{l}\text { Ng, Kankanhalli \& Xu } \\
\text { (2008) }\end{array}$ \\
\hline Perceived severity & Woon, Tan \& Low (2005) \\
\hline $\begin{array}{l}\text { Perceived } \\
\text { susceptibility }\end{array}$ & Ng et al., (2008) \\
\hline Perceived usefulness & Ng \& Rahim (2005) \\
\hline Response efficacy & $\begin{array}{l}\text { LaRose et al. (2008) } \\
\text { Woon et al. (2005) }\end{array}$ \\
\hline Response cost & Woon et al. (2005) \\
\hline Self-efficacy & $\begin{array}{l}\text { Harrington et al. (2006) } \\
\text { LaRose et al. (2008) } \\
\text { Ng \& Rahim (2005) } \\
\text { Ng et al. (2008) } \\
\text { Woon et al. (2005) }\end{array}$ \\
\hline Harrington et al. (2006) \\
\hline Subjective norm
\end{tabular}


relate to each other. The factors which researchers have agreed upon most frequently for influencing cybersecurity intentions are perceived self-efficacy, response efficacy, and the presence of an optimistic bias. However, the general lack of consensus on which factors have the greatest influence on home users' security behaviours indicates that there is a need for further research in this area.

One of the main ways in which researchers have made use of determining the factors that influence cybersecurity intentions is in research on the effectiveness of security messages. When developing security messages it is important to know what factors influence individuals' perceptions of risk. Without understanding these factors, there is a risk that security messages will discourage safe behaviours (LaRose et al., 2008). Research has shown that messages that "focused on the positive consequences" of security behaviours can be more persuasive than those that focus on the negative consequences (Harrington et al., 2006, pp. 1552-1553). When this is looked at in combination with self-efficacy, it seems likely that the reason why positive security messages are more effective is because those messages can increase self-efficacy and have a greater positive influence on users' cybersecurity actions. However, the effectiveness of security messages does not provide a full picture of why users take the steps that they do to protect themselves, if any. This suggests that although security messages may play some role in influencing users' security behaviours, there are likely to be additional factors that need to be identified and studied to determine how security messages and other factors interact to influence users' behaviours.

\subsubsection{Ongoing Protection}

Frequently studies on technology adoption are focused on one-time decisions to utilise protective software. In contrast, behavioural research often focuses on ongoing steps that users take to protect their cybersecurity, for example, always scanning email attachments. While it can be useful to find out what prompts users to adopt particular technologies, there is an assumption that such technologies are "set and forget" and have the potential to be more easily quantified than ongoing behaviours. Instead, it is useful to look at users' overall and ongoing behaviours, part of which may include adopting things like protective technology.

Research on ongoing behavioural change typically focuses on the security practices that users engage in and their reasons for engaging in them. One of the most frequent topics of discussion has been password behaviours. Although passwords can be considered a more technical form of security protection, they are also the subject of ongoing changes. Passwords have an associated set of safe behaviours, including frequently changing passwords, not reusing passwords, and creating secure passwords each time. In this way, 
password behaviours can be looked at as ongoing rather than one-time behaviours.

Continuous awareness and engagement in security behaviours is an important part of cybersecurity because of the changing nature of threats (Dinev \& Hu, 2007). For example, installing antivirus software is only effective until a new set of definitions needs to be installed, and using a password on an account is only effective until that password is compromised. In 2013, 50 million passwords were reset by Evernote as a precaution after the service suffered a security breach that disclosed user names, email addresses, and passwords (Schwartz, 2013). In 2012, over 400,000 passwords were obtained from Yahoo by hackers (Tsukayama, 2012), and another 8 million passwords were leaked from services such as LinkedIn, Last.fm, and eHarmony (Mills, 2012). Password breaches are on the rise, showing why it is so important to ensure that users are not reusing passwords and are changing them regularly, and why it is problematic to assume that setting a password once will provide sufficient protection online.

Though users benefit from ongoing training on creating and using safe passwords (Charoen, Raman, \& Olfman, 2008; Sasse et al., 2004), the biggest issue for home users is that they do not have access to the same sort of training that organisational users receive. In addition, while users are in many cases required to change their passwords at work, unless they realise the benefits of doing so, there is little to encourage users to regularly change their passwords at home. Users often do not change their passwords regularly (Aytes \& Connolly, 2004), and it may not be until a breach occurs that they take steps to do so (Adams \& Sasse, 1999). Therefore, it is important to understand what factors influence users to take ongoing behaviours rather than assuming that security is a one-time event.

\subsubsection{Ongoing Home User Security Behaviours}

From the synthesis of the literature, it becomes clear that a gap on understanding home users' ongoing cybersecurity behaviours still exists. There is a need for examining not only which factors are most relevant to influencing users' cybersecurity intentions, but also to determine how these factors relate to each other. Research has frequently focused on organisational users, leaving a gap in relation to home users' behaviours. Because home users' unsafe behaviours can have a wider effect on the internet as a whole, there remains a need to focus on the factors that influence safer behaviours. Unsafe behaviours can include both unsafe activities (such as clicking on suspicious links, downloading files without ensuring that they are safe, and choosing to ignore warnings) and a lack of safe activities (such as not using antivirus software or firewalls). Both of these types of activities can contribute to breaches on home computers, and as a result, the consideration of unsafe activities needs to include both action and inaction by users. Furthermore, the gap between 
what users know about security and what they do to protect themselves needs to be further explored to determine why, if so many users know how to protect themselves, nearly 20 percent of computers were infected in 2012 (Wilson, 2013). Therefore, to address these gaps, the remainder of the literature review focuses on exploring the factors that have been identified and that will contribute to the conceptual model that is presented in Chapter 3 .

\subsection{Proposed Factors Associated with Cybersecurity Behaviours}

The behavioural approach to cybersecurity has gained traction in recent years with more researchers looking to behavioural models to guide their research. However, the models and theories used have varied widely with little consensus on the most appropriate theoretical approach determine to which factors influence users' security behaviours (see Table 2-3). Tsohou, Karyda, Kokolakis, and Kiountouzis (2006) indicate that there is a need to help decide on "the most appropriate perspective of the theory for the IS security context" (p. 214) which considers stakeholders' perspectives.

Most commonly, researchers have combined the most appropriate parts of various behavioural theories in order to explain users' cybersecurity behaviours. Some of these theories are taken from the health behaviour field, such as the Health Belief Model (HBM) and Protection Motivation Theory (PMT). Health theories such as the HBM and PMT have been useful in exploring why individuals make their decisions about online security (see Dinev \& Hu, 2007; LaRose et al., 2005; Ng et al., 2008). Other research has taken

Table 2-3. Theories used in cybersecurity and other relevant research

\begin{tabular}{|l|l|l|}
\hline Theory & Year & Research \\
\hline Social Comparison Theory & 1954 & Rhee et al. (2005) \\
\hline Rational Choice Theory & 1961 & $\begin{array}{l}\text { Aytes \& Connolly (2004) } \\
\text { Willison (2006) }\end{array}$ \\
\hline Health Belief Model & 1974 & Ng et al. (2008) \\
\hline $\begin{array}{l}\text { Theory of Reasoned } \\
\text { Action }\end{array}$ & 1983 & $\begin{array}{l}\text { LaRose et al. (2005) } \\
\text { Lee et al. (2008) } \\
\text { Woon et al. (2005) }\end{array}$ \\
\hline $\begin{array}{l}\text { Theory of Planned } \\
\text { Behaviour }\end{array}$ & 1985 & $\begin{array}{l}\text { Dinev \& Hu (2007) } \\
\text { Ng \& Rahim (2005) }\end{array}$ \\
\hline $\begin{array}{l}\text { Technology Acceptance } \\
\text { Model }\end{array}$ & 1986 & $\begin{array}{l}\text { Dinev \& Hu (2007) } \\
\text { Ng \& Rahim (2005) }\end{array}$ \\
\hline Social Cognitive Theory & 1986 & $\begin{array}{l}\text { LaRose \& Rifon (2007) } \\
\text { LaRose et al. (2005) } \\
\text { Lee et al. (2008) }\end{array}$ \\
\hline
\end{tabular}


cues from theories such as Coping Theory in order to create new theories to explain what motivates users' cybersecurity behaviours (see Liang \& Xue, 2009; 2010).

Table 2-4 provides an overview of the main factors included in the identified theories and shows the main areas where there are overlapping factors. These factors have been used to guide the remainder of the literature review and to examine the findings of previous cybersecurity-related research. The factors identified can be grouped into six main areas: (1) perceived threat, (2) personal factors, (3) demographic factors, (4) outside influences, (5) perceived barriers and benefits, and (6) outcomes.

Perceived threat includes perceptions about users' susceptibility to threats and the severity of the consequences. The combination of severity and susceptibility into perceived threat comes from the HBM and PMT. Personal factors include factors that are unique to the individual. The personal factors explored are: perceived self-efficacy, attitude, the subjective norm, perceived usefulness and ease of use, trust, awareness, knowledge,

Table 2-4. Identification of main factors from theories that have been used in cybersecurity research

\begin{tabular}{|c|c|c|c|c|c|c|c|c|c|c|c|c|c|c|c|c|c|c|c|}
\hline & 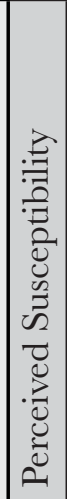 & 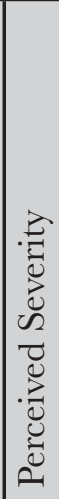 & 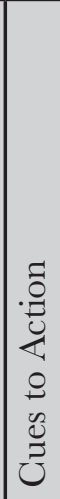 & 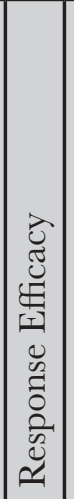 & 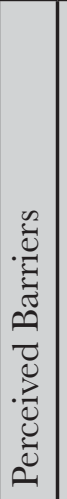 & 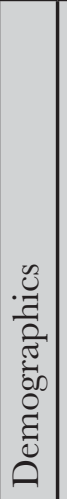 & $\left|\begin{array}{c} \\
0 \\
0 \\
0 \\
0 \\
0 \\
3 \\
0 \\
0 \\
0 \\
0\end{array}\right|$ & 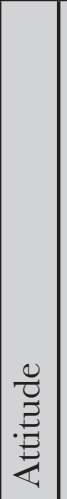 & 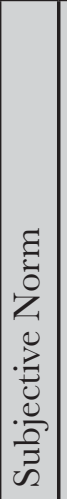 & 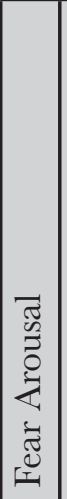 & 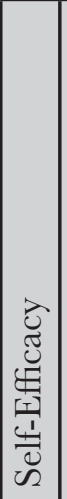 & 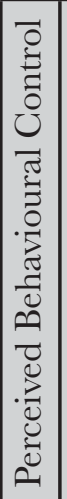 & 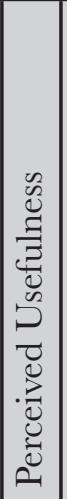 & 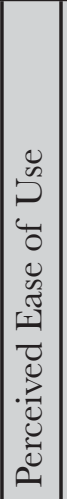 & 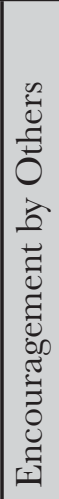 & 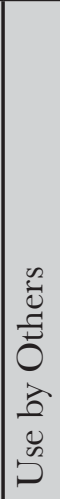 & 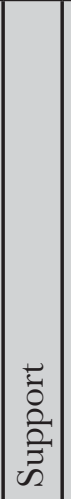 & $\mid$ & $\frac{\vec{U}}{\frac{u}{x}}$ \\
\hline $\begin{array}{l}\text { Health Belief } \\
\text { Model }\end{array}$ & & & & & & & & & & & & & & & & & & & \\
\hline $\begin{array}{l}\text { Theory of } \\
\text { Reasoned Action }\end{array}$ & & & & & & & & & & & & & & & & & & & \\
\hline $\begin{array}{l}\text { Protection } \\
\text { Motivation Theory }\end{array}$ & & & & & & & & & & & & & & & & & & & \\
\hline $\begin{array}{l}\text { Theory of Planned } \\
\text { Behaviour }\end{array}$ & & & & & & & & & & & & & & & & & & & \\
\hline $\begin{array}{l}\text { Technology } \\
\text { Acceptance Model }\end{array}$ & & & & & & & & & & & & & & & & & & & \\
\hline $\begin{array}{l}\text { Social Cognitive } \\
\text { Theory }\end{array}$ & & & & & & & & & & & & & & & & & & & \\
\hline $\begin{array}{l}\text { Computer Self } \\
\text { Efficacy Theory }\end{array}$ & & & & & & & & & & & & & & & & & & & \\
\hline $\begin{array}{l}\text { Used in } \\
\text { Cybersecurity } \\
\text { Research }\end{array}$ & & & & & & & & & & & $V$ & $V$ & & $X$ & & & & & \\
\hline
\end{tabular}


security and risk orientation, and personal experience with cybersecurity breaches. Outside influences refer to factors that are in the individuals' external environment. The outside factors identified in the literature were cues to action, encouragement by others, and use by others. Perceived barriers and benefits focus on the factors that inhibit and enable users' security behaviours. Finally, outcomes focus on users' intentions and behaviours. These factors, their included sub-factors, and the surrounding literature, form the basis of the conceptual model presented in the next chapter.

\subsubsection{Perceived Threat and Perceived Risk}

Barnes, Bauer, Neuman, and Huber (2007) suggest that an individual's actions "are only triggered when this individual's limit of tolerance is being crossed" (p. 75). Barnes et al (2007) discussed this in relation to the uncertainty and consequences associated with a consumer purchase not meeting the customer's needs. However, the idea that actions cannot be triggered without an individual considering the risk or threat to be relevant, and with severe enough consequences, that they can no longer tolerate the risk is one that has been commonly explored in security research.

Perceived threat originally comes from the Health Belief Model (HBM). In the HBM, perceived threat has been defined as "the subjective perception of the risk of contracting a condition" (Janz \& Becker, 1984, p. 2). This perception is based on the perceived severity of the consequences and the perceived susceptibility to the threat (see Figure 2-2). A similar concept exists in Protection Motivation Theory (PMT), termed threat appraisal. Threat appraisal is defined as the process of evaluating "the factors that increase or decrease

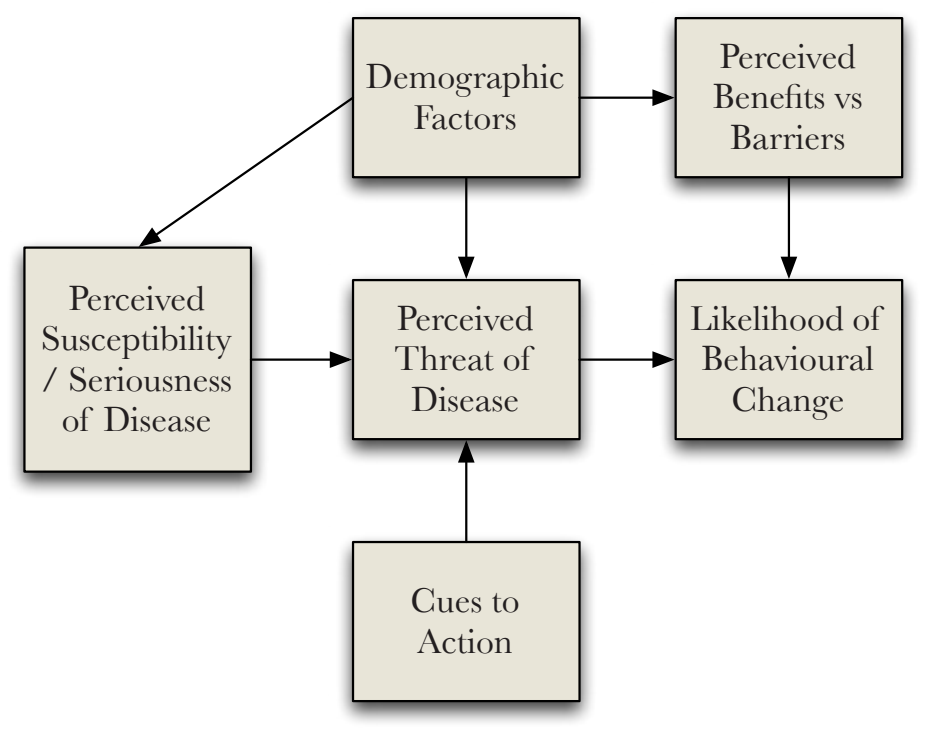

Figure 2-2. Health Belief Model the likelihood of enacting protective behaviours, changing undesired behaviours, or both" (Neuwirth, Dunwoody, \& Griffin, 2000, p. 722). In PMT, threat appraisal is comprised of vulnerability, fear arousal, and severity (Figure 2-3, p. 33). The individual components of threat appraisal are defined later in the relevant sections.

A related concept of perceived risk was added to the Technology Acceptance Model 
(TAM) and examined in a study on the use of online applications. In that study, perceived risk was defined as "the degree to which a user feels the uncertainty and adverse consequences of using an online application service" (Lu, Hsu, \& Hsu, 2006, p. 109). Although this concept was associated with the perceived

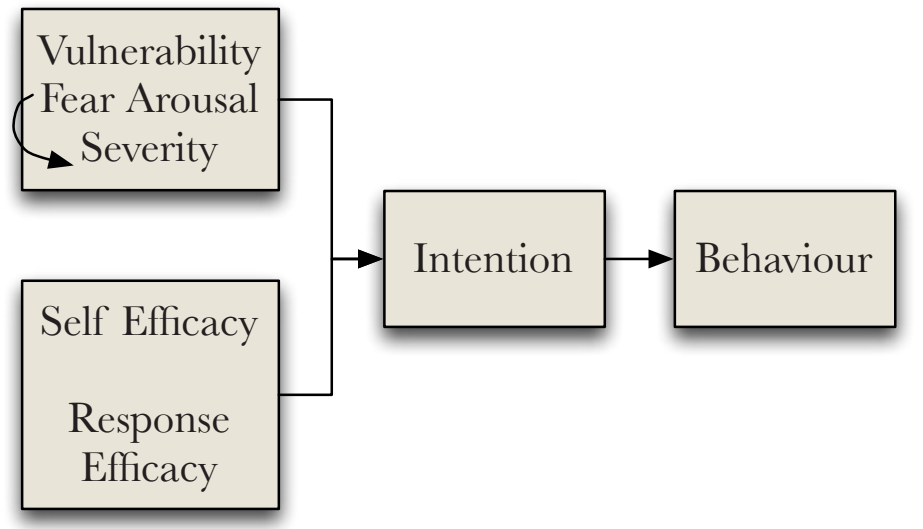

Figure 2-3. Protection Motivation Theory risk of purchasing goods online, the definition can be looked at more broadly to consider whether uncertainty contributes to the threat or risk that users feel by using the internet. Risk perception has also been considered in conjunction with online purchasing and it was found that lower levels of trust led to higher levels of risk perception and that higher levels of risk perception led to a decreased likelihood of buying online (Jarvenpaa, Tractinsky, \& Vitale, 2000). These studies support the addition of risk to existing models, such as TAM, which did not originally account for risk.

Liang and Xue (2009; 2010) developed a model for exploring cybersecurity intentions as part of their Technology Threat Avoidance Theory (TTAT) which included perceived threat. In their study, perceived threat was defined as "the extent to which an individual perceives the malicious IT as dangerous or harmful" (Liang \& Xue, 2010, p. 397). They found that higher levels of perceived threat had a positive effect on motivating threat avoidance behaviours. However, they did not explore what made users believe that malicious IT was dangerous or harmful.

Although perceived threat was used separately from perceived severity and perceived susceptibility in the HBM, in almost many cases the individual components of perceived threat include severity, susceptibility, and with PMT, fear arousal. Therefore, while it is useful to consider perceived threat as an overall combination of users' perceptions, the majority of the literature focuses on the effects of the individual components. The components of perceived severity, perceived susceptibility, fear arousal, and safeguard / response efficacy are explored in the subsequent sections.

\subsubsection{Perceived Severity of Cybersecurity Breaches}

Perceived severity is common to both the HBM and PMT. Perceived severity has been defined as a person's "evaluations of both medical/clinical consequences... and 
possible social consequences" (Janz \& Becker, 1984, p. 2). The concept of perceived severity has been used in the cybersecurity context to refer to "a person's perceived seriousness of a security incident [that is] not limited to damage to systems and data, but also the implications on the person's job or organisation" ( $\mathrm{Ng}$ et al., 2008, p. 2). Although the definition has already been adapted to cybersecurity use, it needs to be re-defined in the context of home users for whom there may not be job related consequences, but instead more personal consequences.

Perceived severity along with perceived susceptibility are two of the most commonly researched factors influencing cybersecurity behaviours. In Aytes and Connolly's (2004) research into the rational choice to engage in safe practices, they utilised a predictive model for information use based on the Theory of Reasoned Action (TRA) and TAM. Although their model included the concept of "perceived negative consequences," they were unable to predict from this factor how often a person would engage in security behaviours because respondents did not believe that the likelihood of experiencing these consequences was high enough to cause concern.

Woon et al.'s (2005) study on wireless network security settings made use of PMT. In their study, they showed that perceived severity was a much greater motivator for individuals to use security settings than perceived vulnerability was. They suggested that the reason why perceived severity is a more effective motivating factor in cybersecurity research than it is in health research is due to the fact that "the impact [of threats] is more uniform" whereas in health, there are a number of individual factors which can impact perceptions of severity.

In a study on organisational users' cybersecurity behaviours using a variation on the HBM, Ng et al. (2008) found that perceived severity had positive and negative moderating effects on the other factors. Higher levels of perceived severity increased the impact of cues to action and general security orientation in relation to users' cybersecurity behaviours. However, higher levels of perceived severity also decreased the positive effects of selfefficacy and individual perceptions about the effectiveness of security behaviours. However, these researchers did not examine how the factors used influenced each other and how they may be further related.

Liang and Xue (2009; 2010) studied spyware and the use of anti-spyware software. In their research, they examined the effects of perceived severity and perceived susceptibility on the overall perceived threat. They found that greater levels of perceived severity led to greater levels of perceived threat. They also found that although both perceived severity and perceived susceptibility contribute to the perceived threat, there was no interaction effect between the two factors. Furthermore, they found that perceived threat mediated 
perceived severity and susceptibility's influence on avoidance motivation.

Overall, past research has predominantly found that perceived severity has contributed to an increased perception of threat by users. However, it is unclear from the previous research what factors contribute to users' perceptions of severity. Therefore, although it seems clear that perceived severity is a relevant factor in exploring cybersecurity behaviours, there is still a need to identify how individuals' intentions to engage in cybersecurity behaviours are formed and what factors can increase or decrease the perception of severity of consequences associated with cybersecurity breaches.

\subsubsection{Perceived Susceptibility to Cybersecurity Breaches}

Another factor common to the HBM and PMT is perceived susceptibility. Perceived susceptibility has been defined in the HBM as a person's "subjective perception of the risk of contracting a condition" (Janz \& Becker, 1984, p. 2). In PMT, the term vulnerability is used and has been described as an individual's "estimate of the chances of contracting the disease" (Plotnikoff \& Higginbotham, 2002, p. 88). The idea of perceived susceptibility has been used in the security context to refer to "a user's perceived likelihood of a security incident taking place" (Ng et al., 2008, p. 5). Perceived susceptibility can be increased through fear arousal, a concept from PMT (Johnston \& Warkentin, 2010). Witte and Allen's (2000) meta-analysis of 98 studies with "at least two levels of a fear appeal" showed that historically, "the stronger the fear appeal, the greater the fear aroused, the greater the severity of the threat perceived, and the greater the susceptibility to the threat perceived" (p. 597). This suggests a strong relationship between messages that use fear appeals and increased perceptions of severity and susceptibility.

Similar to perceived severity, perceived susceptibility has been found to have a positive effect on perceived threat (see Liang \& Xue, 2010) and on computer security behaviours ( $\mathrm{Ng}$ et al., 2008). However, the main issue with perceived susceptibility as a factor motivating users' security behaviours is that users may not have strong feelings of susceptibility. One way to increase feelings of susceptibility is through the use of fear appeals, which trigger fear arousal. Without the sense of susceptibility, people are unlikely to believe there is a sufficient threat to take preventative actions.

Fear arousal refers to the "unpleasant emotional state triggered by the perception of threatening stimuli” (Ruiter, Abraham, \& Kok, 2001, p. 614). Fear arousal can be triggered by fear appeals, which are "persuasive communication [s] attempting to arouse fear in order to promote a precautionary motivation and self-protective action" (Ruiter et al., 2001, p. 614). However, there are doubts about the effectiveness of fear appeals and their ability to motivate protective behaviours (Ruiter et al., 2001). In order for a fear appeal to be 
successful, there are three criteria that need to be met: "(1) the problem is serious and may affect the person; (2) it can be avoided by taking appropriate action; and (3) the person is capable of performing the necessary behaviour required to avoid the problem" (Weirich \& Sasse, 2001, p. 140). Anderson and Agarwal (2010) found that greater concern over threats which relates to the severity and relevance of threats was more likely to create "a positive attitude about taking action" (p. 822).

The concept of vulnerability has been studied in terms of individuals comparing their perceived level of vulnerability to that of their peers. Rhee et al. (2005) found that people often thought they were less susceptible to threats than their peers and often demonstrated an optimistic bias. Davinson and Sillence (2010) studied the effect of education on users' security behaviours in relation to phishing. Davinson and Sillence (2010) found that respondents considered their peers to be significantly more susceptible to phishing emails than they considered themselves. People also often felt that they were not susceptible to password-based security threats because: (1) they were unlikely to be targeted by malicious users, (2) the damage caused would not be severe, and (3) they could not with certainty prevent all possible threats (Weirich \& Sasse, 2001). A decreased sense of vulnerability can lead to users engaging in less cautious behaviours online (Workman, 2007). There is also the possibility of a pessimistic bias, which exists when people have unrealistically low estimates of their ability to cope with situations (Blanton, Axsom, McClive, \& Price, 2001). Although the idea of pessimistic bias has been explored in psychology, it does not appear to have been explored in the cybersecurity context.

Perceived susceptibility has often been researched alongside perceived severity. Although researchers have considered the interplay between severity and susceptibility (for example, Liang \& Xue, 2010), there has been little consideration of the factors that influence these perceptions of severity and susceptibility. While the HBM does provide additional insight into the factors that influence perceived threat, it is unclear in the cybersecurity context which of these factors are most relevant and if there are any additional factors that are unique to the cybersecurity context.

\subsubsection{Safeguard / Response Efficacy}

The effectiveness of safeguards or protective behaviours can further influence users' perceptions of severity and susceptibility. The effects of protective steps in motivating users' behaviours come from PMT, the HBM, and Social Cognitive Theory (SCT) (see Figure 2-4). From PMT, response efficacy refers to "the individual's expectancy that carrying out the recommendations can remove the threat" (Plotnikoff \& Higginbotham, 2002, p. 88). Within the HBM, the concept of perceived benefits address "belief in the efficacy or value 
of a behavior in reducing the threat" (Becker, Maiman, Kirscht, Haefner, \& Drachman, 1977, p. 349). A similar concept found in Social Cognitive Theory (SCT) is that of expected outcomes. Expected outcomes are judgements about "the likely consequences that a behaviour will produce" (Bandura, 1986, p. 391).

Perceived benefits have been defined in computer security research as "a user's

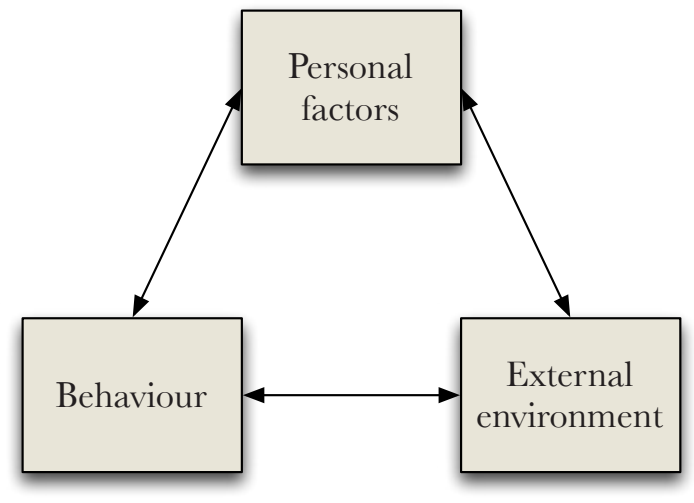

Figure 2-4. Social Cognitive Theory belief in the perceived effectiveness of practicing computer security" (Ng et al., 2008, p. 819); for example, beliefs about whether the use of anti-virus software will prevent cybersecurity breaches. Perceived benefits were found to have a positive influence on users' cybersecurity behaviours, in particular, taking care when opening email attachments (Ng et al., 2008).

Liang and Xue (2010) called this factor safeguard effectiveness and defined it as "the subjective assessment of a safeguarding measure regarding how effectively it can be applied to avoid the IT threat" (p. 399). They further note that safeguard effectiveness is similar to outcome expectancy from SCT, perceived benefits in HBM, and response efficacy in PMT. In their research, they found that safeguard effectiveness had a positive effect on avoidance motivation (Liang \& Xue, 2010). However, when perceived threat was looked at in conjunction with safeguard effectiveness, there was a negative interaction effect. They found that greater levels of perceived safeguard effectiveness decreased perceived threat, which in turn resulted in lower levels of threat avoidance motivation and that the reverse was true where there were lower levels of perceived threat.

One potential issue that arises in examining the effect of perceived benefits is that the effectiveness of the safeguards may not always be clear. It is much more difficult to find a tangible benefit to cybersecurity behaviours compared to health behaviours since with cybersecurity the primary benefit is keeping the status quo and the "non-occurrence of events" (Chan, Woon, \& Kankanhalli, 2005, p. 21). This can also make it difficult to judge whether safeguards are actually effective. It may also mean that peoples' judgements about safeguard efficacy are based more on more subjective personal factors rather than on an objective view of the safeguard's effectiveness. Therefore, there is a need to look further into the personal factors that influence perceptions about safeguard efficacy. 


\subsubsection{Personal Factors}

Personal factors refer to cognitive and affective factors that are unique to the individual. Seven possible personal factors associated with cybersecurity were identified based on the literature: perceived self-efficacy, attitude, the subjective norm, perceived usefulness and ease of use, trust, awareness, and knowledge.

\subsubsection{Perceived Self-Efficacy and Cybersecurity}

Perceived self-efficacy is defined in SCT as “people's judgements of their capabilities to organize and execute courses of action required to attain designated types of performances" and is concerned with "the judgements of what one can do with whatever skills one possesses" (Bandura, 1986, p. 391). Self-efficacy has been added to the HBM in order to make it more applicable to situations requiring long-term behavioural changes, which were not addressed in the original HBM (see Rosenstock, Strecher, \& Becker, 1988). Self-efficacy is also included in PMT as part of the coping appraisals, which are judgements about individuals' beliefs about the response costs, response efficacy, and self-efficacy (Neuwirth et al., 2000). Coping appraisals were found to be a better predictor of security behaviours than threat appraisals when predicting privacy behaviours in teenagers (see Wirth, Rifon, LaRose, \& Lewis, 2007).

Ajzen (1991) refers to perceptions about how easy or difficult it is to do something as perceived behavioural control in the Theory of Planned Behaviour (TPB) (Figure 2-5). Perceived behavioural control "reflect[s] past experiences as well as anticipated impediments

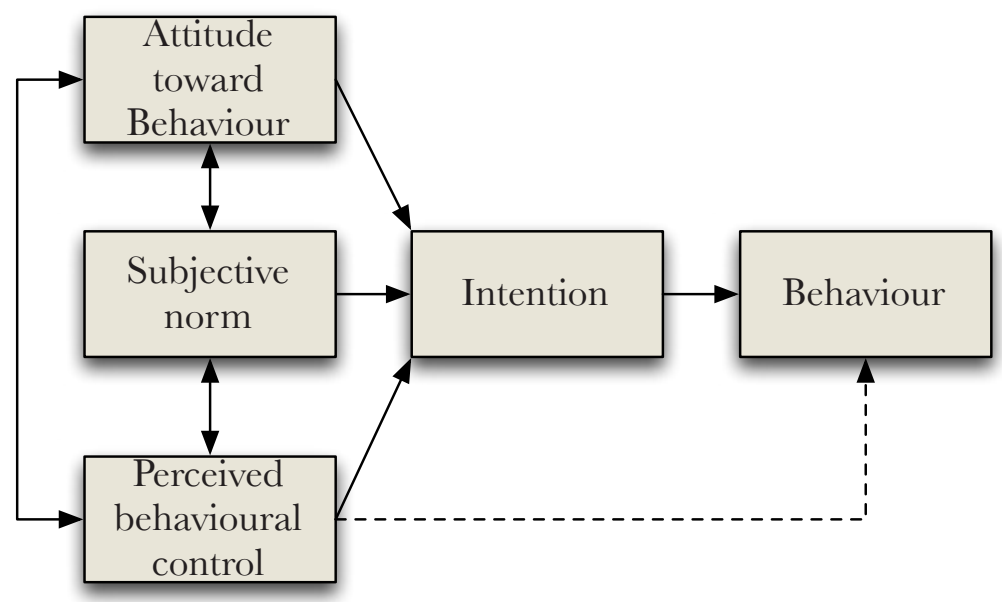

Figure 2-5. Theory of Planned Behaviour and obstacles" (Ajzen, 1991, p. 188). Fishbein and Cappella (2006) have indicated that perceived behavioural control is a determinant of selfefficacy.

Within IS literature, self-efficacy has been studied in the context of computer use, leading to the development of the Computer Self Efficacy 
Theory (CSET) (Figure 2-6). Computer self-efficacy is defined as "a judgment of one's capability to use a computer" and is concerned with broad computer-related tasks (Compeau \& Higgins, 1995b, p. 192). Furthermore, computer self-efficacy applies to "efficacy across multiple computer application domains" rather than applying to a single skill (Marakas, Yi, \& Johnson, 1998, p. 129). While computer self-efficacy has not been specifically used in cybersecurity research, it provides additional clarification on how the more general concept of self-efficacy can be applied in the computing domain. It also provides insight into factors that can influence perceived self-efficacy, such as encouragement by others, use by others, and support (see Compeau \& Higgins, 1995a). The definition of computer self-efficacy has been used as a foundation for defining "self-efficacy in information security" which refers to individuals" beliefs that they are able to "protect information and information systems from unauthorized disclosure, modification, loss, destruction, and lack of availability" (Rhee, Kim, \& Ryu, 2009, p. 818).

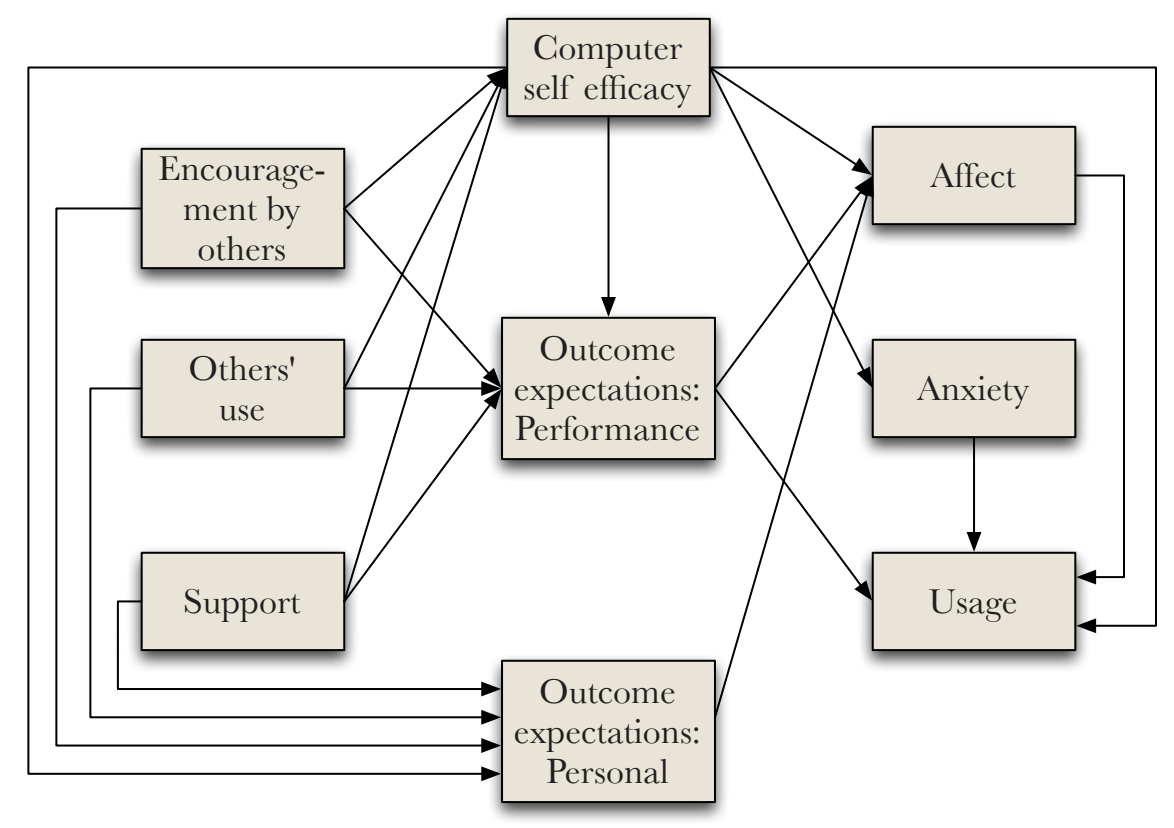

Figure 2-6. Computer Self Efficacy Theory

Self-efficacy has been shown to be an accurate predictor of future behaviours within the short term, although not always in the long term (Rhodes \& Plotnikoff, 2005). Ajzen (1991) described how a "person who is confident that he can master [an] activity is more likely to persevere than [a] person who doubts his ability" (p. 184). Although Ajzen was speaking of behavioural control, the idea that people who believe in their ability to achieve a particular outcome will be more likely to persevere with it is similar to the concept of selfefficacy. Overall, it seems that perceived self-efficacy is especially important in influencing 
cybersecurity behaviours when a person has recently learned about a threat, experienced a threat, or learned about a new way to protect themselves online.

Research into user behaviours relating to wireless security showed that self-efficacy was not only a good predictor of greater intention to use wireless security, but it was also closely linked to knowledge (Woon et al., 2005). Woon et al. (2005) found that people who took steps to enable wireless security features themselves had greater levels of knowledge as well as greater levels of perceived self-efficacy compared to individuals who had someone else set up the security features. This indicates that knowledge may also be a driver of perceived self-efficacy.

In an organisational study on the effect of security climate on secure behaviours, Chan et al. (2005) found that both security climate and self-efficacy contributed to security "compliant" behaviour. They further state that "compliant behavior is dependent on a combination of organizational and personal factors" but that there were additional factors that needed to be identified in order to better explain compliant behaviour (p. 36). The organisational features included in that study were upper management practices, direct supervisory practices, and co-worker socialisation. Although the organisational factors identified in Chan et al.'s study are not directly applicable to the context of home user cybersecurity behaviours, these factors can provide some insight into whether there is a personal security climate dictated by other computer users within the household, and may further contribute to the understanding of the subjective norm.

In Ng and Rahim's (2005) study on home users' intentions to protect themselves online, they found that perceived self-efficacy had a positive influence on perceived behavioural control. However, they found limited support for their hypothesis that perceived behavioural control would have a positive influence on intention to engage in safer behaviours. In a later study on organisational users' security behaviours, Ng et al. (2008) found that selfefficacy had a positive effect on intention to practice safe email behaviours, but that the effect of self-efficacy diminished as the perceived severity of the threat increased. This suggests that while self-efficacy may be important, the effects of it may be limited. However, it should be noted that perceptions about individuals' competence may be affected by their desire to be seen as friendly (for example, see Fisk, Guddy, \& Glick, 2007; Holoien \& Fiske, 2012, Kervyn, Judd, \& Yzerbyt, 2009). Therefore, there is a risk that perceptions about individuals' abilities to protect themselves may be different than their actual skill and knowledge.

Although self-efficacy has frequently been included in research on cybersecurity behaviours, areas remain where further research is possible. In particular, further research is needed on the value of self-efficacy in relation to other factors that influence behaviours, and factors that can influence self-efficacy in the cybersecurity context. While Compeau 
and Higgins (1995a) identified encouragement by others, others' use, and support as influencing computer self-efficacy, it remains to be seen if these factors are still applicable in the personal cybersecurity context or if there are additional factors that may be useful.

\subsubsection{Attitude and Cybersecurity}

Attitude is "a person's favorable or unfavorable evaluation of an object or action" (Fishbein \& Ajzen, 1975, p. 12) and is present in the Theory of Reasoned Action (TRA) (see Figure 2-7) and TPB (Figure 2-5, p. 38). According to Fishbein and Ajzen (1975) an individual's attitude is influenced by beliefs, which "represent the information he has about the object" (p. 12). Attitude has primarily been used in examining the use of ethical computing behaviours rather than computer security behaviours.

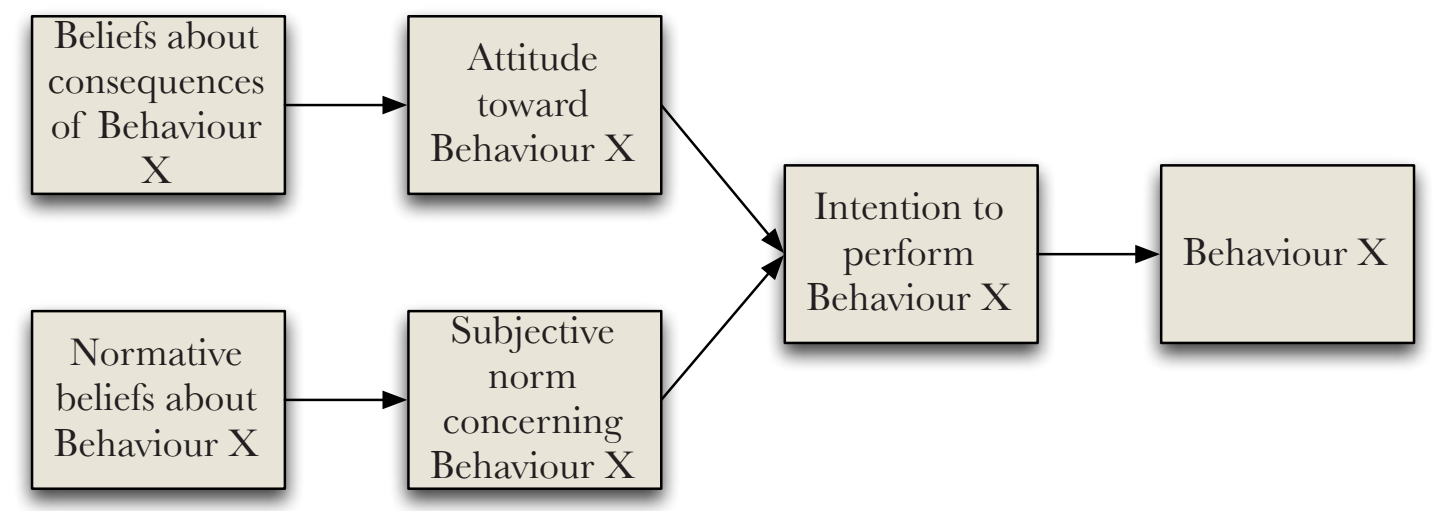

Figure 2-7. Theory of Reasoned Action

Lee and Lee (2002) proposed a model for predicting computer abuse. Based on a combination of TPB, General Deterrence Theory, Social Bond Theory, and Social Learning Theory, they suggested that attitude toward computer abuse would be influenced by attachment to the persons' referents, their involvement in "conventional activities", their commitment to actually committing computer abuse, and beliefs about computer abuse (Lee \& Lee, 2002, p. 59). Furthermore, they suggested that computer abuse intention was influenced by attitude, subjective norm, and perceived behavioural control. The focus of their study was on predicting behaviours where either "a victim suffered, or could have suffered, a loss, and a perpetrator made, or could have made a gain" (Parker, 1998, p. 333 in Lee \& Lee, 2002). Although the context of the study is different, the research on computer abuse behaviours shows how attitude may influence behaviours and what factors 
could influence attitudes.

The intention to behave ethically or unethically has also been studied. Leonard, Cronan, \& Krele (2004) found that attitude to act ethically or unethically could be explained by “consequences, personal values, one's belief system, society, laws, professional codes of ethics, and the personal environment components" (p. 150). Although not all of the factors identified by Leonard et al. were relevant in this study, the factors of consequences, personal values, one's belief system, society, and personal environment had the most relevance.

\subsubsection{The Subjective Norm and Cybersecurity Behaviours}

The subjective norm comes from TRA (Figure 2-7, p. 41) and TPB (Figure 2-5, p. 38). It is defined as a person's "beliefs that certain referents think the person should or should not perform the behavior in question" (Fishbein \& Ajzen, 1975, p. 16). When applying this to the cybersecurity context, it refers to users' beliefs that people in their social circle feel that they should be utilising protective behaviours. Armitage \& Conner (2001) examined 185 behavioural studies that used TPB and found the subjective norm was one of the weaker factors that influenced individuals' intentions.

In a study on computer abuse, Lee and Lee (2002) proposed that intention to engage in computer abuse in the organisation is in part influenced by the subjective norm. Furthermore, they proposed that the subjective norm is influenced by co-workers and senior management. The main issue in applying this factor to the home user context is that home users may not have people in their social circle who are influencing their decision to undertake protective behaviours.

Dinev and $\mathrm{Hu}$ (2007) found that people were less likely to seek out peer approval about their computing behaviours than about other consumer issues. They also found that the subjective norm had a much greater influence on users who were considered to be more advanced because advanced users were much more likely to talk about computer issues and "seek solutions" within their social circles (p. 401). Therefore, the amount of experience, skill, and awareness that an individual has in relation to computer security may impact whether the subjective norm is influential in users' security behaviours.

\subsubsection{Perceived Usefulness \& Ease of Use of Safeguards}

Perceived usefulness and ease of use come from TAM (Figure 2-8). Perceived usefulness is "the prospective user's subjective probability that using a specific application system will increase his or her job performance within an organisational context" (Davis, 
Bagozzi, \& Warshaw, 1989, p. 985). Perceived ease of use is "the degree to which the prospective user expects the target system to be free of effort" (Davis et al., 1989, p. 985). Perceived usefulness and ease of use influence a person's intention to use a technology, which in turn influences actual system use. Although these factors were originally studied in the organisational context, perceived ease of use can easily be used in the home context, while perceived usefulness can be adapted to further support the idea of safeguard / response efficacy by looking at the usefulness of the software in reducing security threats.

The TAM and TPB have been used together to create a model which focuses on the effect of technology awareness to illustrate intention to use protective technologies (Dinev \& Hu, 2007). Dinev and $\mathrm{Hu}$ (2007) found that perceived ease of use and perceived usefulness were not as meaningful as the fear of dire consequences in influencing people to use anti-spyware software. However, as is discussed in 2.4.4.1, the use of fear as a motivator can be questionable.

The potential weakness with using perceived usefulness as a construct is that there often is not a clear benefit to engaging in protective behaviours. The main benefit of engaging in these behaviours is keeping the status quo and the "non-occurrence of events" (Chan et al., 2005). This often means that the benefits of cybersecurity behaviours are not clear. More often, people see when the security steps they have taken have failed, rather than when they have succeeded. In this way, it can become difficult for individuals to judge the usefulness of protective behaviours.

While ease of use is important when studying, for example, a piece of software, it is of less importance in this research because of the focus on human behaviours. Although human behaviours are inevitably tied to the adoption of technology, the focus here is not on what makes a piece of technology useful or not. Rather, the focus is on the individual who in turn needs to be confident enough in their skills to want

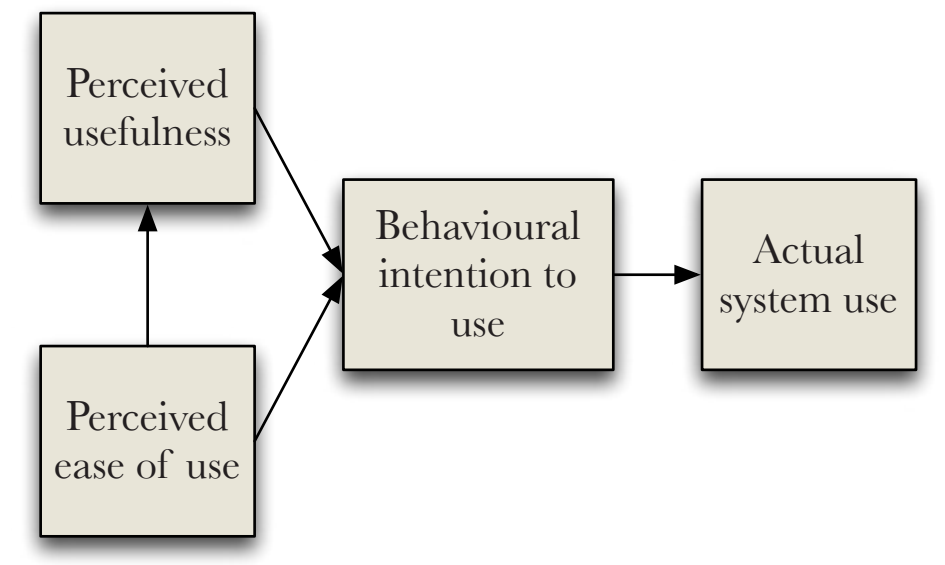

Figure 2-8. Technology Acceptance Model to attempt to use the software before they actually do so. As a result, an individuals' perceived self-efficacy is likely to be a more appropriate focus in this study, rather than usability. 


\subsubsection{Trust}

The concept of trust has often been included in research on the use of e-commerce technologies (see Jarvenpaa et al., 2000; Lee \& Turban, 2001; McKnight, Choudhury, \& Kacmar, 2002; Verhagen, Meents, \& Tan, 2006). However, in recent years, individual perceptions of trust have been used in the security context. Sund (2007) suggested that in order for internet use to continue to grow, trust was needed, but that trust was steadily declining due to security issues. Chen and Bansal's (2010) proposed model suggested that trusting belief is a precursor to intention to transact with a website. They defined trusting belief as existing when "the truster (the end user) perceives that the trustee (e.g. a specific web site) has attributes that are beneficial to the user" (Chen \& Bansal, 2010, p. 3). Although they related trust to a specific website, trusting belief could be further expanded to relate to people that users interact with online, web sites, or other online communications. Junglas, Johnson, and Spitzmüller (2008) suggest that people who are more agreeable are more likely "to trust others and be less suspicious of their environment or other individuals (Costa et al., 1991)" (p. 391). They found that the more agreeable people were, the less concerned they were about their privacy (Junglas et al., 2008). Although this was related to privacy, it can applicable to security issues as well due to the closeness in their nature.

\subsubsection{Awareness and Cybersecurity}

Awareness has some of its roots in Innovation Diffusion Theory (ITD). According to IDT, awareness is "the extent to which a target population is conscious of an innovation" (Dinev \& Hu, 2007, p. 390). However, there is a risk that home users will be less cognizant of the benefits of an innovation such as protective software when using a home computer than they would be if they were using it in an organisation where training about the benefits would be available (Dinev \& Hu, 2007). In studying the use of protective technologies, Dinev and $\mathrm{Hu}$ (2007) noted that individuals need to be aware of the existence of the protective technology but that their awareness of it is tempered by their perception of a need for it. These researchers found strong support for awareness being one of the main factors influencing the formation of intention and attitudes.

Users also need to be aware of messages that indicate whether sites are safe, which may be a form of a security cue. Chen and Bansal (2010) proposed the inclusion of security awareness in their integrated model on individual web security behaviour. They defined security awareness as the "awareness of security information / cues on the site's vulnerability" which includes "specific site security information and security indicators" (p. 
4). These indications of whether a site is safe may be related to users' safeguard awareness as well as their knowledge because checking for these clues would be a security behaviour that requires a certain level of understanding of what makes a site safe or unsafe.

Judging intention to practice security based solely on awareness may be problematic because awareness of security does not require individuals to act. Aytes and Connolly (2004) found that users who felt they are sufficiently educated about security, regardless of their actual education on the topic, were unlikely to try to improve their knowledge. Their study suggested that awareness campaigns that informed users about security procedures were not enough to get users to actually protect themselves as their respondents considered themselves to be aware of the threats and safeguards, yet often engaged in risky behaviours (Aytes \& Connolly, 2004). Within the privacy context, Drennan, Mort, and Previte (2006) studied privacy risk perceptions of home users and found that awareness of privacy threats did not have a positive effect on risk perception. However, their study did show that increased perceptions of privacy risk were positively related to online privacy behaviours.

\subsubsection{Knowledge / Training / Skill}

Knowledge about security has been shown to influence how individuals describe cybersecurity threats (Rifon, Quilliam, \& LaRose, 2005). Rifon et al (2005) found that internet users who considered themselves "newbies" often focused on "dire consequences" and their fears (p. 4). In contrast, they found that internet users who considered themselves "confident" more often focused on what they had learned from their past experiences and how those experiences made them take more steps to protect themselves (Rifon et al, 2005). Knowledge and skill can be obtained through specific training (e.g. in the workplace) or it may be self-led. Training about internet security has been shown to increase awareness of such behaviours (see Aytes \& Connolly, 2004). A survey of 167 university students by Aytes and Connolly found that over half of their respondents considered themselves to be knowledgeable, very knowledgeable, or experts about email behaviours, preventing viruses, preventing computer crashes, and preventing disclosure of financial information (2004). However, even with these high levels of knowledge they found that 20 to $30 \%$ of their respondents engaged in risky computing behaviours, such as sharing passwords or opening email attachments without scanning them, at least occasionally. Although it was unclear what role knowledge had in influencing users' security behaviours, knowledge may influence other factors indirectly. This gap between what users know and what they do to protect their security is an area that requires further research. 


\subsubsection{Security / Risk Orientation}

$\mathrm{Ng}$ et al. (2008) adapted the concept of "health orientation" from the HBM (Figure 2-2, p. 32) to "security orientation" in the internet security context. They define security orientation as "a user's predisposition and interest concerning practicing computer security" (p. 819). They found that although security orientation did not have a direct effect on security intentions, it operated with perceived severity and cues to action to have a positive influence on computer security behaviours (Ng et al., 2008). Security and risk orientation has not been extensively researched in the security context. Therefore, it is unclear if security or risk orientation influences security intention directly or if it has a more indirect influence, such as by affecting attitude as was shown by $\mathrm{Ng}$ et al. (2008).

\subsubsection{Previous Personal Experiences}

Previous personal experience has been added to several studies on users' internet security behaviours. Aytes and Connolly (2004) used a rational choice model to explore risky behaviours among individuals. In that model, they included personal experience as a antecedent to awareness of safe practices and of negative consequences. However, from their study it was unclear what effect personal experience had on users' decisions. Chen and Bansal (2010) proposed an integrated model for studying security behaviours which included personal experience as part of an individual's personal characteristics. In their study, they indicated that personal experience can be positive, for example making a successful purchase online, or negative, for example being the victim of phishing scam (Chen \& Bansal, 2010).

Results have been mixed as to the effect of previous experience on users' online behaviours. Harrington et al. (2006) considered whether a person had been personally affected by a virus and whether the person knew someone else who had been affected by a virus. In their research on computer safety messages they found that previous experience, whether personal or vicarious, did not have a significant effect on intention to practice safe computing (Harrington et al., 2006).

\subsubsection{Gender and Age}

Dommeyer and Gross (2003) found that men were more aware of privacy strategies than women. Gender has also been shown to relate to fear responses, especially with crime. 
Women tend to have higher levels of personal fear of being victims of crime (see LaGrange \& Ferraro, 1989; Warr \& Ellison, 2000). Cybercrime and traditional crime are increasingly approached similarly in terms of individuals' perceptions of fear. Therefore, it may mean that gender influences users' cybersecurity behaviours.

Age has not been shown to have a direct relation to cybersecurity behaviours. Within a privacy context, Dommeyer and Gross (2003) hypothesised that younger people would be more aware of privacy because of the amount of attention paid to it by the media. However, these researchers found that the opposite was true. They also found that younger users were more likely to engage in privacy protection behaviours (Dommeyer \& Gross, 2003). A study by Sheehan (2002) who developed a privacy typology based on privacy concern, showed that older users were either unconcerned about their privacy or highly concerned, while younger users were more pragmatic about privacy. Based on the privacy literature, it was unclear whether age would influence users' cybersecurity behaviours and how it would do this.

\subsubsection{External Influences}

External influences have also been identified as relating to users' cybersecurity behaviours. Often in the organisational context, these external sources are related to policies, training, and organisational security expectations. However, in the home user context, these factors need to be considered differently since many of the external influences present for organisational users are not present for home users. Both cues to action and encouragement and use by others are possible external influences because they can be applied in both home and organisational environments.

\subsubsection{Cues to Action}

Gues to action come from the HBM (Figure 2-2, p. 32) and refer to the stimulus necessary to trigger the decision to engage in a protective behaviour (Janz \& Becker, 1984, pp. 2-3). In the HBM, cues to action is comprised of symptoms, knowledge, and media information. In the context of this thesis, knowledge was considered as an internal factor because it related more to the individual's general knowledge about computers and security and was not limited to a single threat. Therefore, symptoms and media information are considered in this section as aspects of cues to action.

In the original HBM, symptoms are considered internal (Janz \& Becker, 1984). However, in cybersecurity they are external because they apply to the computer rather 
than the individual. LaRose et al. (2005) suggested that computers may act as extensions of ourselves, as well as "extensions of our family or social circles" and that most people equated their relationship with their computer to a relationship with another human (p. 3). In this way, although symptoms may not be internal, the way that we view our computers suggests that labelling the consequences of cybersecurity breaches as symptoms may be an appropriate analogy.

$\mathrm{Ng}$ et al. (2008) found that cues to action did not have a significant effect on intention to practice safer computing behaviours. However, in their research, they also note that their focus was on awareness programmes as a trigger. They go on to state that "other forms of cues to action, such as individual experience or other forms of communication external to the organisation" may still be relevant (p. 823). As discussed in section 2.4.1, awareness campaigns regularly focus on triggering fear arousal and it is often difficult to trigger the appropriate responses because users frequently lack the belief that they are vulnerable or that the consequences of a breach would be severe. Therefore, although awareness campaigns may not trigger users' security behaviours, there may be other cues that do. Without these cues, users may have an inflated "sense of invulnerability" (Johnston \& Warkentin, 2010, p. 561). This may in turn lead to lowered perceptions of severity, susceptibility, and overall threat. Therefore symptoms and media information remain relevant to this research.

Closely linked to awareness campaigns is media information. Mass media can be used to present awareness campaigns such as those run by internet security organisations or government departments, for example cybersecurity awareness month (see Department of Homeland Security, n.d.) or Netsafe.org.nz's security basics campaign (see NetBasics, 2008). While the media may provide useful information, questions remain about how much of it is considered relevant by individuals when it is done as an awareness campaign. In a home environment where organisational policies and training do not exist, the media is likely to be the most prominent source of information about cybersecurity threats. Media stories may trigger greater concern about threats which prompt users to act. The media can cause "sudden shifts in behaviour... [termed] 'social amplification (or attenuation) of risk"” (Neuwirth et al., 2000). In addition to the media, there may also be other external sources that prompt users to engage in safer cybersecurity behaviours.

\subsubsection{Encouragement \& Use by Others}

Users may be influenced to take more steps to protect themselves through encouragement by others to use protective technologies and take protective steps. They may be further influenced to protect themselves if they see other people taking these steps 
themselves. Encouragement by others and use by others were originally used in CSET (Figure 2-6, p. 39). Encouragement by others refers to encouragement by "others within the individuals' reference group" and "represents 'verbal persuasion' one of the four main sources of efficacy information" (Bandura, 1986 as cited in Compeau \& Higgins, 1995b, p. 195). Use by others refers to "the actual behavior of others with respect to the technology" and focuses on "learning by observation" (Compeau \& Higgins, 1995b, p. 195). While neither of these factors have specifically been used in cybersecurity research, both can increase individuals' perceived self-efficacy and were therefore relevant to this research.

\subsubsection{Barriers to Security Behaviours}

Perceived barriers are taken into consideration in both the HBM (Figure 2-2, p. 32) and PMT (Figure 2-3, p. 33), though their implementation is different. In the HBM, perceived barriers to change are a person's "perceptions that [the actions] may be expensive, dangerous, unpleasant, inconvenient, time-consuming, and so forth" (Janz \& Becker, 1984, p. 2). In the HBM, perceived barriers are considered alongside perceived benefits. However, as discussed in 2.4.1.3, perceived benefits as defined in the HBM relate more closely to a combination of response-efficacy and self-efficacy.

In PMT, Neuwirth et al. (2000) identified three factors that decreased the effectiveness of the threat appraisal process and the coping appraisal process: extrinsic rewards, intrinsic rewards, and response barriers. Extrinsic rewards are the rewards such as "social approval" for continuing a maladaptive behaviour while intrinsic rewards refer to rewards such as pleasure for continuing a non-preventive behaviour (Neuwirth et al., 2000, p. 722). Response barriers decreased the coping appraisal process (Neuwirth et al., 2000). Response barriers are the closest to perceived barriers in the HBM, and refer to the costs, time, and effort associated with engaging in a preventive behaviour (p. 722). Although these factors were proposed to decrease threat and coping appraisal, Neuwirth et al.'s (2000) research did not support this.

Perceived barriers have been considered in other research into internet security by $\mathrm{Ng}$ et al. (2008). They defined perceived barriers as "a user's perceived cost and inconvenience of practicing computer security" and suggested that these barriers would decrease the likelihood of an individual taking protective steps (p. 5). Although $\mathrm{Ng}$ et al. (2008) found that perceived barriers did not have an effect on intention to engage in security behaviours in their study, they indicated that this may have been related the fact that their respondents were "part-time (working) computer students and/or work[ing] in IT related organisations" and therefore would not have difficulty practicing the security behaviours being studied 
(p. 823).

Research so far has not shown that perceived barriers lower the likelihood of an individual practicing preventive behaviours. However, the preventive behaviours considered in the HBM and PMT of cost, time, and inconvenience may be more prevalent in a study on home user security behaviours. Unlike organisational users, home users are required to bear the time and energy costs to ensure that their system is safe. In contrast, security is largely invisible for organisational users, who for the most part already have security software installed and are not responsible for the associated maintenance (Dinev \& $\mathrm{Hu}$, 2007). Because of this, barriers may play a greater role in preventing home users' security behaviours.

\subsection{Summary of the Literature \& Research Gap}

Previous literature on users' cybersecurity behaviours indicated that these behaviours could be influenced by perceived threat, internal factors, external factors, demographic factors, and perceived barriers and benefits. Perceived threat consisted of perceived severity, perceived susceptibility, and perceived safeguard effectiveness. Internal factors identified in the literature included perceived self-efficacy, attitude, the subjective norm, perceived usefulness and ease of use, trust, awareness, knowledge, security orientation, and previous personal experience. In addition to these internal factors, external factors such as cues to action, encouragement, and use by others could also influence users' security behaviours. Furthermore, these behaviours could also be influenced by demographic factors. Finally, perceived barriers and benefits can hinder or encourage individuals' intentions to engage in cybersecurity behaviours.

By identifying the factors that researchers have previously studied it was clear that there has been little agreement on the factors that influence users' cybersecurity behaviours (see Table 2-5, p. 51-52). The influence of self-efficacy, perceived severity, and perceived susceptibility have been well established in cybersecurity research, although at times with varying results. Self-efficacy has consistently been shown to have a positive influence on intention to engage in safer behaviours online, as has perceived susceptibility. However, the effects of perceived severity on intention have been mixed. Outside of these main factors, it remains to be seen what other factors have a significant influence on cybersecurity, and several gaps in the literature remain.

Although research on home users has increased in recent years, there are still gaps when it comes to examining how certain factors may affect home users differently from organisational users. In particular, studies on organisational users which have indicated that certain factors did not have a significant effect on users' intention have indicated a 
Table 2-5. Summary of factors examined in previous literature relevant to users' cybersecurity behaviours

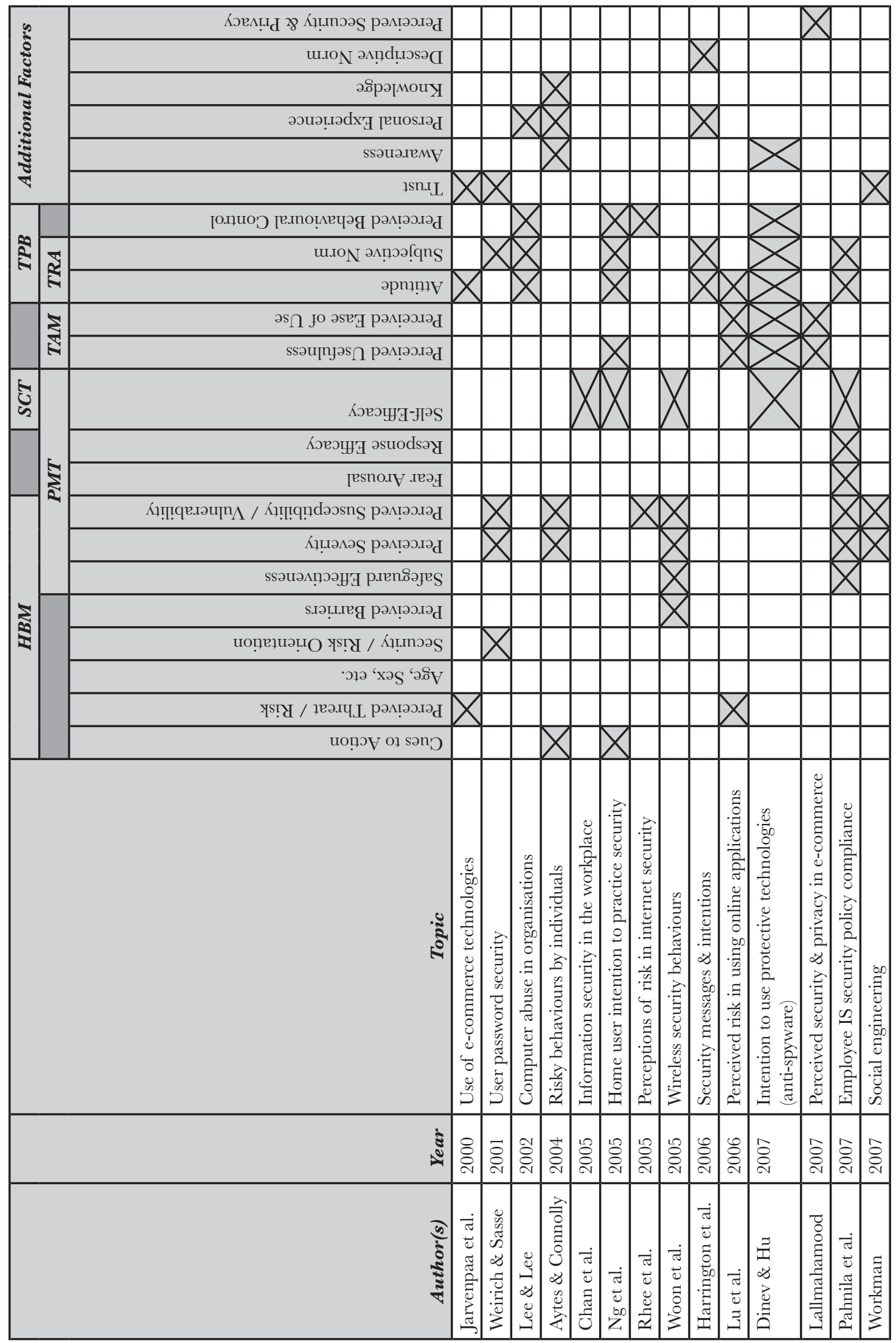




\begin{tabular}{|c|c|c|c|c|c|}
\hline 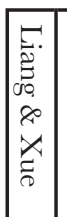 & 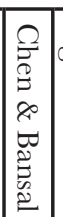 & $\left|\begin{array}{cc}r & 3 \\
0 & 0 \\
0 & 0 \\
0 & 0 \\
0 & 0 \\
0 & 0 \\
0 & 8 \\
& 8\end{array}\right|$ & 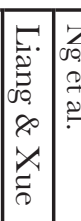 & $\sqrt{\frac{a}{2}}$ & \\
\hline $\begin{array}{l}\stackrel{N}{0} \\
0\end{array}$ & \begin{tabular}{|l|l}
0 \\
0 \\
0
\end{tabular} & $\begin{array}{l}\mathfrak{0} \\
\stackrel{0}{0}\end{array}$ & 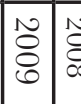 & $\begin{array}{l}3 \\
3 \\
3\end{array}$ & \\
\hline 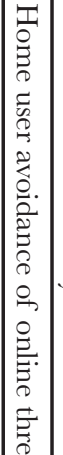 & 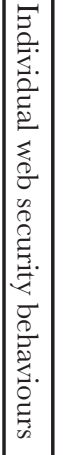 & 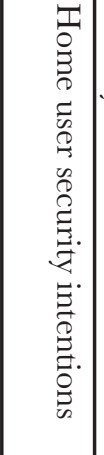 & 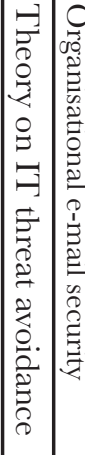 & . & \\
\hline & & & & Cues to Action & \\
\hline & X & & X & Perceived Threat / Risk & \\
\hline & & & & Age, Sex, etc. & \\
\hline & & & & Security / Risk Orientation & $\equiv$ \\
\hline$\bigotimes$ & & & X & Perceived Barriers & $\frac{1}{2}$ \\
\hline Х & & & $x$ & Safeguard Effectiveness & \\
\hline 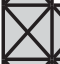 & X & & $x x$ & Perceived Severity & \\
\hline X & X & & Xx & Perceived Susceptibility / Vulnerability & \\
\hline & & & & Fear Arousal & 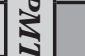 \\
\hline & & & & Response Efficacy & \\
\hline y & & & V & Self-Efficacy & 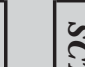 \\
\hline & X & & & Perceived Usefulness & \multirow{2}{*}{5} \\
\hline & X & & & Perceived Ease of Use & \\
\hline & & & & Attitude & \\
\hline & & & & Subjective Norm & \\
\hline & X & & & Perceived Behavioural Control & \\
\hline & & & & Trust & \multirow{6}{*}{ है } \\
\hline & X & & & Awareness & \\
\hline & X & & & Personal Experience & \\
\hline & X & & & Knowledge & \\
\hline & & & & Descriptive Norm & \\
\hline & & & & Perceived Security \& Privacy & \\
\hline
\end{tabular}


need for similar research on home users' to see if the outcome would differ (see $\mathrm{Ng}$ et al., 2008). In addition, there remains a need to research users' security behaviours rather than focusing purely on adoption of technology because it assumes that "doing security" is a one time event rather than an ongoing set of behaviours.

Identifying the personal factors associated with users' security behaviours has shown the need for additional research. While factors have been identified, it is unclear in many cases how these factors relate to each other and if there are additional factors that could be included. While nine possible personal factors have been identified based on the literature, it is unclear if all of these factors are relevant to home user security behaviours. Therefore, there is a need to further study the factors influencing users' perceptions of threat and the effect of awareness on home users' intention to engage in security behaviours, as these areas have not yet been fully explained.

Finally, there remains a need to explore how the factors identified can contribute to the understanding of the gap between what people know and what they do. By understanding what factors contribute to this gap, it may be possible to take steps to reduce it. It appears that in many cases users consider themselves to be knowledgeable enough about security to protect themselves. However, research shows that computer security remains an issue and that we have yet to conclusively determine why people do not take more steps to protect themselves online.

\subsection{Chapter Summary}

This chapter illustrated why there is a need to investigate users' cybersecurity behaviours and the factors that influence these behaviours. As indicated it is unclear which factors are particularly relevant to explaining users' cybersecurity behaviours. This demonstrated the need to develop a conceptual model to guide the research. Chapter 3 examines the methods employed to collect and analyse the data used to answer the research questions and presents the conceptual model based on the literature. 


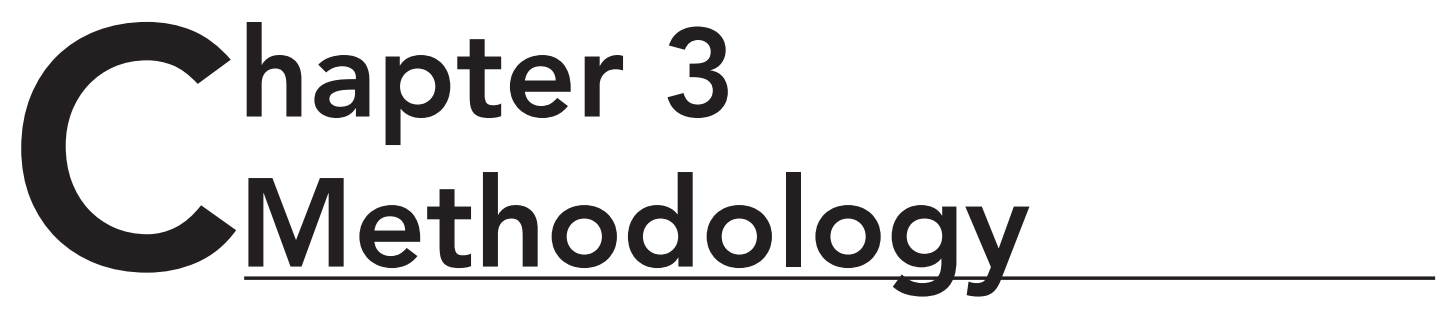

\subsection{Introduction}

This chapter addresses the research paradigm, methodology, data collection, and data analysis procedures. In this exploratory study, a series of interviews and focus groups was used to explore the factors related to home users' security behaviours, which were guided by a conceptual model that is presented in this chapter. The qualitative data were analysed in accordance with the interpretive paradigm. After the selection of research method is explained and justified, this chapter presents the data collection and analysis procedures.

\subsection{Overview of Paradigm, Approach, and Methods}

Three commonly used research paradigms in information management are positivist, interpretivist, and critical. Positivist research tests theory by examining fixed relationships between constructs (Chua, 1986; Orlikowski \& Baroudi, 1991). Interpretivist research seeks to "understand the deeper structure of a phenomenon" and obtain a "shared understanding of the phenomenon" (Orlikowski \& Baroudi, 1991, p. 5). Finally, critical 
research critiques the current state of the research and attempts to reveal the "historical, ideological, and contradictory nature of existing social practices" (Orlikowski \& Baroudi, 1991, p. 5).

Chapter 2 explored the current state of the research and showed how there is yet to be a cohesive understanding of not only what cybersecurity is, but what factors influence users' intentions to engage in cybersecurity practices. Guided by this, an interpretivist approach was used to better understand the phenomenon of cybersecurity behaviours in greater detail. While qualitative research has been done on cybersecurity behaviours in the past, much of the literature has focused on testing existing theories and their suitability for use in the security domain. Therefore, it was necessary to utilise an interpretive paradigm to help identify potentially new factors related to cybersecurity behaviours and to gain a better understanding of the topic overall.

\subsubsection{Exploratory Research}

As indicated in the previous chapter, there is little consensus about what factors contribute to home users' cybersecurity behaviours. Combined with the relative newness of the field, this suggested that there was a need to explore which of the existing factors were relevant and to identify potential new factors for deeper exploration. Therefore, an exploratory approach was taken in this research. Exploratory research can be used to "gain insights and to discover new ideas" (Zikmund, 1987, p. 40).

Exploratory research is useful for generating theory about cybersecurity behaviours, an emerging topic. The objectives of exploratory research are "theory initiation and theory building" (Onwuegbuzie \& Leech, 2005, p. 278). Despite exploratory research being well suited to new areas of study, such as cybersecurity, one danger is that the findings of exploratory research are difficult to generalise and "may not be representative of the population being studied" (Singh, 2007, p. 64). Although difficult to generalise, the purpose of exploratory research is to gain a greater understanding of the phenomenon, making it suitable for this study.

\subsubsection{Qualitative Approach}

A qualitative approach was taken in this study in order to get insight into additional factors that influenced individuals' cybersecurity behaviours. One of the limitations of previous cybersecurity research has been the emphasis on the use of quantitative methods. The lack of agreement on which factors influence users' cybersecurity behaviours and 
how they do so suggests that there is room for additional research into other factors which further contribute to users' intentions to engage in protective behaviours. Qualitative data has a "richness and holism with strong potential for revealing complexity" that may not be present in quantitative research (Miles \& Huberman, 1994, p. 10).

Qualitative research focuses on getting data in a way that allows us to understand "what 'real life' is like" (Miles \& Huberman, 1994, p. 10). It is defined as research that does not include statistical findings and that lets "the "phenomenon of interest unfold naturally" (Patton, 2001, p. 39, as cited in Golafshani, 2003, p. 600). Much of the existing cybersecurity research has focused on determining if users' security behaviours can be explained using the constructs of already established theories. However, there has been little agreement by researchers about what is the most appropriate theoretical basis to use. This lack of agreement indicated a need to build on the existing theory and to determine the most relevant parts for the context in order to better address the drivers of cybersecurity behaviours.

Few studies have taken a qualitative approach to determining the factors influencing users' cybersecurity behaviours. Miles and Huberman (1994) advocate qualitative research as the "best strategy for discovery, exploring a new area, [and] developing hypotheses" (p. 10). Given the lack of cohesive approaches to explaining cybersecurity behaviours (Table 2-3, p. 30), a qualitative approach appeared to be well suited for further exploration of the factors influencing users' cybersecurity behaviours.

\subsubsection{Interpretive Paradigm}

Researchers have acknowledged that users play an important role in protecting cybersecurity because they have the ability to override the technological protections installed on their computers (see Lineberry, 2007; Sasse et al., 2004). However, the factors influencing these behaviours are not yet thoroughly understood. Much of the home user oriented cybersecurity research to date has been undertaken using quantitative, positivist methods (for example, see Aytes \& Connolly, 2004; Dinev \& Hu, 2007; Furnell et al., 2007; LaRose et al., 2008; LaRose et al., 2007; LaRose et al., 2005; Ng \& Rahim, 2005). However, little agreement has been reached on which factors influence users' intentions to engage in security behaviours. This suggests a need to explore other factors that influence users' behaviours.

An interpretive approach is especially appropriate when the focus is on understanding the context of the research problem and the complexity associated with human understanding (Klein \& Myers, 1999). Because cybersecurity inherently involves the interaction of users and technology, it is not sufficient to look at either one or the other. Instead, they should be 
considered together. Lee (2001, as cited in Gregor, 2006) states "research in the information systems field examines more than just the technological system, or just the social system, or even the two side by side...it investigates the phenomena that emerge when the two interact" (p. 613). It is this interaction that contributes to the complexity of understanding the phenomenon.

A positivist paradigm is more appropriate for proving or disproving theory, while the interpretivist paradigm uses theory to sensitise the researcher to a particular way of viewing the world (Klein \& Myers, 1999). Cybersecurity research is relatively new and there is a shortage of research into home users' security behaviours. This indicated a need to explore the topic in a way that enabled participants to bring their own understanding to the topic and to have these concepts integrated into the research. Therefore, an interpretivist paradigm was better suited to gaining the required understanding of the phenomenon and the factors associated with users' behaviours which could later be tested.

When using the interpretive paradigm, there is an assumption that reality comes from social constructions and cannot be understood without the actors who create the reality (Klein \& Myers, 1999; Orlikowski \& Baroudi, 1991). It is important to understand what the threats are and how they can be countered. However, without understanding the perceptions and behaviours associated with addressing threats, we are faced with an incomplete picture of what influences users' security behaviours. The simple presence of protective technology is not enough to ensure that people will use it. With cybersecurity behaviours, it is not just the technology that dictates what individuals do, though the technology available influences users' behaviours. Interpretive research in the IS context combines an understanding of the system as well as "the process whereby the information system influences and is influenced by the context" (Walsham 1993, pp. 4-5, as cited in Klein \& Myers, 1999, p. 69). Cybersecurity research inherently brings together the user and the technology and looks at how they interact.

\subsubsection{Use of Focus Groups and Interviews}

Focus groups were used in the first stage of the data collection. Focus groups are made of up groups of individuals who have personal experience on a topic (Gibbs, 1997). What distinguishes focus groups from other types of qualitative data gathering is the interaction that happens between participants (Gibbs, 1997). The use of focus groups allowed for the development of a shared understanding of the topic as well as an understanding of how group dynamics influenced respondents (Basch, 1987). Furthermore, focus groups allowed for a greater level of consensus to be obtained (Gibbs, 1997). Therefore, focus groups provided an appropriate starting point for exploring users' cybersecurity intentions and 
behaviours as well as the drivers of these.

The second stage of the research was to conduct individual interviews. Interviews are particularly helpful when the researched behaviours of participants cannot be directly observed (Creswell, 2003). Semi-structured interviews were used in order to allow for flexibility in the questions while still starting with an idea of the areas for discussion (Myers \& Newman, 2007). Semi-structured interviews allowed for new issues to be explored in greater detail. They also allowed for the topics raised in early interviews to inform the questions asked of later interviewees.

While respondents could have demonstrated their behaviours in particular situations using an experimental approach, this research focused on their reasons for engaging in cybersecurity behaviours, which cannot be observed. In addition if the respondents had been studied in a lab situation, it may have biased the way they acted or put a greater focus on the behaviours than the factors influencing these behaviours. In addition, a controlled environment could have prevented respondents from discussing events not included in an experiment. Therefore, interviews enabled the provision of a winder scope for discussing cybersecurity drivers and issues, especially those that were not already identified through the existing literature.

Before the data was collected, a conceptual model was developed in order to guide the research. In the next section, the conceptual model based on the literature is presented and explained.

\subsection{Conceptual Model Development}

The analysis of the literature and the associated models provided a number of factors of particular relevance in researching cybersecurity behaviours and the factors that influence them. These factors were:

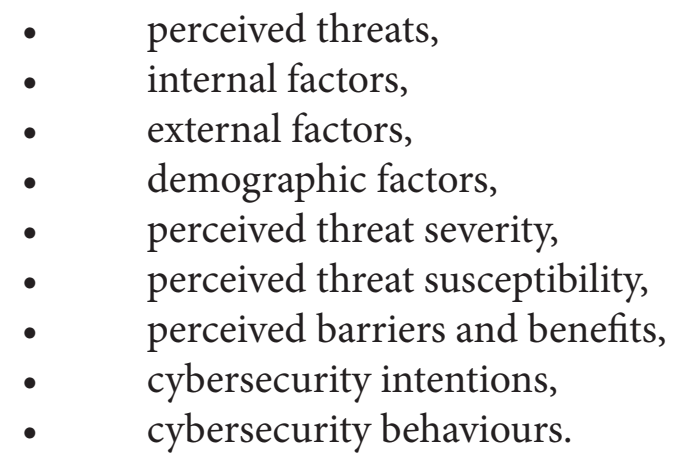

Each group of factors and the proposed relationships with other factors are discussed in the subsequent sections. These will be followed by the conceptual model which was developed based on the existing literature. 


\subsubsection{Perceived Threat}

Perceived threat comes from health theory, and often refers to the perceived threat of a disease. This concept can be further adapted to the cybersecurity context to refer to the perceived threat of a cybersecurity attack. Perceived threat is the combined perceptions about the severity of and susceptibility to a particular threat. Perceptions of threat may be influenced by internal and / or external factors. From the literature, it is expected that users who have lower levels of perceived threat will be less likely to intend to engage in cybersecurity behaviours and therefore, not engage in as many protective behaviours as a user with higher levels of perceived threat.

\subsubsection{Perceived Susceptibility}

Perceived susceptibility to threats refers to the perceived likelihood of a cybersecurity breach occurring. There is a need to consider the perceived susceptibility to threats as the past literature showed that users often did not feel that it was likely they would have to face breaches. This belief often caused users to believe that the perceived severity of threats was lower than it may have actually been.

\subsubsection{Perceived Severity}

Within the cybersecurity context, perceived severity focuses on the data, system, and personal consequences of a breach. Past cybersecurity research found that perceived severity had a moderating effect on perceived susceptibility, perceived benefits, perceived barriers, cues to action, security orientation, and self-efficacy ( $\mathrm{Ng}$ et al., 2008). This suggests that perceived severity may not have a direct effect on perceived threat, but may instead work in conjunction with perceived susceptibility.

\subsubsection{Proposed Internal Factors}

Six internal factors were identified from the literature: subjective norm, prior experience, knowledge, security orientation, perceived self-efficacy, and attitude (see Figure 3-1, p. 61). Internal factors are expected to influence users' perceptions of severity, threats, barriers, and benefits. In addition to these factors, there may be other internal 
factors which influence users' behaviours. Each of the identified factors is discussed below.

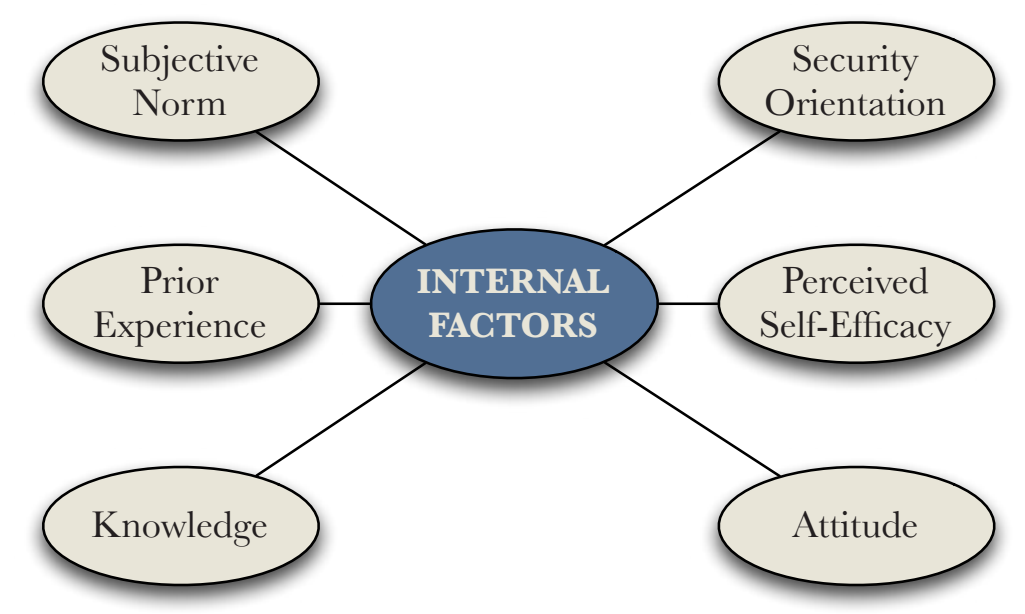

Figure 3-1. Proposed internal factors influencing individuals to engage in cybersecurity behaviours

\subsubsection{Subjective Norm}

The subjective norm comes from the TRA (Figure 2-7, p. 361) and TPB (Figure 2-5, p. 358) and refers to individuals' beliefs about how they believe that other people feel they should act (Fishbein \& Ajzen, 1975). Researchers have had mixed results on the impact of the subjective norm and how it influences cybersecurity behaviours. Based on conflicting findings between Ng and Rahim (2005) and Dinev and $\mathrm{Hu}$ (2007), it is currently unclear if and how relevant this factor will be in the current study.

\subsubsection{Prior Experience}

Prior experience can relate to the amount of internet experience a person has and whether the individual has ever had negative experiences with cybersecurity. More advanced users were likely to use protective technologies even if the benefits of using those technologies were not immediately clear (Dinev \& Hu, 2007). Based on this, it is expected that individuals who have been using the internet longer will be more likely to be aware of the benefits of using safeguards.

It is unclear from the literature whether prior negative experience with data loss, malware, or fraud may cause individuals to be more cautious in their behaviours. Good, Grossklags, Thaw, Perzanowski, Mulligan, and Konstant (2006) conducted a study on 
user installation of malware in relation to end user license agreements (EULAs). They found that some respondents were more cautious about what they installed because of past negative experiences with malware and phishing. In contrast, Harrington et al. (2006) found that in relation to the effectiveness of warning messages, "prior experience with a security violation" did not have a significant influence on attitude or intention to practice safe computing. However, it may still be possible that it has an effect on other factors or acts as a mediating factor.

\subsubsection{Knowledge}

Knowledge of cybersecurity threats and the possible consequences may also influence users to engage in safer practices (see Rhee et al., 2005). Health literature suggests that knowledge and awareness can come from several sources including awareness campaigns, friends and family, experts in the field, or the media (Janz \& Becker, 1984). Knowledge about the available threats may have an impact on the internal factors that influence cybersecurity behaviours because it is necessary that individuals be knowledgeable enough to identify a threat.

\subsubsection{Security Orientation}

Security orientation is "a user's predisposition and interest concerning practicing computer security" (Ng et al., 2008, p. 5). Although Ng et al. found that a general security orientation did not directly impact cybersecurity behaviours, it may be possible that an individual's security orientation has a less direct impact on their behaviours and instead influenced their intentions indirectly.

\subsubsection{Perceived Self-Efficacy}

Perceived self-efficacy refers to how capable individuals consider themselves to be in relation to engaging in particular actions to get expected outcomes (Bandura, 1986). Similarly, computer self-efficacy refers to how capable individuals consider themselves to be at using computers (Compeau \& Higgins, 1995b). Individuals may also have beliefs about their self-efficacy in relation to their ability to protect themselves online. Engaging in cybersecurity behaviours requires some knowledge of the threats and how these threats can be countered. Because of this, users may be more likely to believe that they are capable of 
protecting themselves if they already have higher levels of knowledge. Therefore, perceived self-efficacy may be influenced by both technical and behavioural actions.

\subsubsection{Attitude}

Attitude is a person's positive or negative feelings about an object or action (Fishbein \& Ajzen, 1975). The action in this case would refer to a user engaging in cybersecurity behaviours. Individuals may have both positive and negative attitudes towards the behaviours in question, which may in turn affect their intention to engage in the protective behaviours.

\subsubsection{Proposed External Factors}

Cues to action, encouragement by others, and use by others have been identified as potential external factors that influence users' intention to engage in cybersecurity behaviours (see Figure 3-2). From the literature, three main external factors were identified: cues to action, encouragement by others, and use by others. These factors were a starting point for identifying factors in the user's external environment.

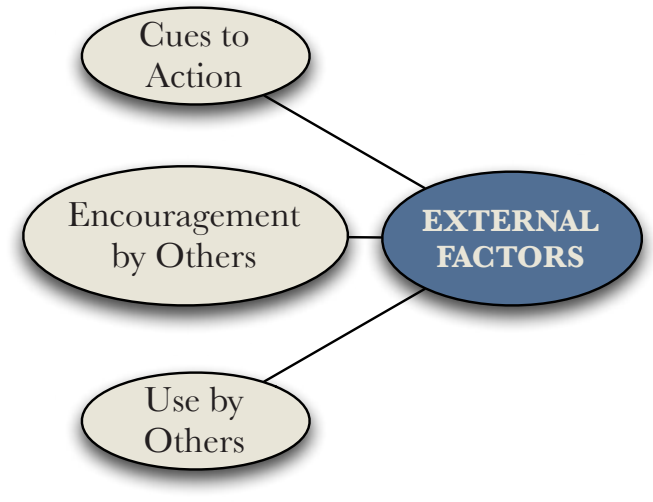

Figure 3-2. Proposed external factors influencing individuals to engage in cybersecurity behaviours

\subsubsection{Cues to Action}

Within a health context, cues to action take into account symptoms, knowledge, and media information about threats (Glanz, Rimer, \& Lewis, 2002). In the cybersecurity context, the symptoms refer to symptoms that can be seen on a computer, such as slowed performance or other tangible symptoms. For the purposes of this research, knowledge is considered as a separate internal factor because it relates directly to the individual and is not necessarily specific to a single cybersecurity threat, but rather the individuals' knowledge of computers, the internet, and security in general and how that relates to their intentions. 


\subsubsection{Encouragement \& Use by Others}

Encouragement by others and use by others are both from the CSE model (see Compeau \& Higgins, 1995b). Encouragement and use by friends and family has been shown to influence the subjective norm in regards to cybersecurity (see Ng \& Rahim, 2005). Encouragement by others is the "encouragement of use [of a technology] by others in the individual's reference group and use by others refers to "the actual use of computers by others in the individual's reference group (Compeau \& Higgins, 1995b, p. 194). Although these concepts refer to the use of technology, they can also be applied to engaging in cybersecurity behaviours. These factors may be particularly relevant when a computer is shared between several people, such as families or individuals living together in shared accommodation.

\subsubsection{Demographic Factors}

In past research, age and gender have had mixed results in terms of influencing users' security and privacy behaviours. Therefore, they have been included in the conceptual model and have been considered along with the other identified factors.

\subsubsection{Perceived Barriers \& Benefits}

Perceived barriers and benefits have been examined from the perspective of both positive and negative behaviours in health theory. The HBM (Figure 2-2, p. 352) defines barriers and benefits of engaging in positive behaviours. According to the HBM, perceived barriers are an individual's perceptions that it may be expensive, dangerous, unpleasant, inconvenient, time-consuming, and so forth" to take steps to prevent or treat a disease, while perceived benefits are the "beliefs regarding the effectiveness of the various actions available in reducing the disease threat" (Janz \& Becker, 1984, p. 2). It is expected that individuals' perceptions about the barriers to engaging in safe practices and their beliefs about the efficacy of safeguards will influence their intention to engage in safer practices online. 


\subsubsection{Cybersecurity Intentions}

User's cybersecurity intentions are expected to be influenced by the perceived threat, perceived barriers, and perceived benefits. Furthermore, a user's intentions are expected to impact their security behaviours. According to the TRA (Figure 2-7, p. 361), there is an assumption that people will engage in a behaviour as long as they have a strong enough intention to do so. However, previous researchers identified a gap between what users know about cybersecurity and what they do to protect themselves (for example, see Aytes \& Connolly, 2004; Furnell et al., 2007; Furnell et al., 2008). This raises a question of whether individuals accurately express their intention to engage in cybersecurity behaviours.

\subsubsection{Cybersecurity Behaviours}

Cybersecurity behaviours are the intended outcome in the conceptual model. Ng and Rahim (2005) indicated that there was no agreed upon list of safe behaviours online. This means that individual behaviours will need to be considered against a selection of common security behaviours that are not necessarily exhaustive, but provide a starting point from which to judge whether a person is taking some appropriately safe steps. Examples of such safe behaviours come from security sites such as Netsafe.org.nz, Microsoft, and US-CERT and included:

- not sharing passwords,

- using strong passwords,

- not opening email attachments without being certain they do not contain malicious code,

- using protective software,

- ensuring computer software is patched and up to date, and

- ensuring networks and browsers are secure.

\subsubsection{Conceptual Model}

Based on these identified concepts from the literature, a conceptual model was developed (see Figure 3-3, p. 66 and Table 3-1, p. 67-68). While the model integrates a number of concepts identified from previous cybersecurity literature and theories, it has been heavily influenced by the HBM. The conceptual model illustrates the expectation 


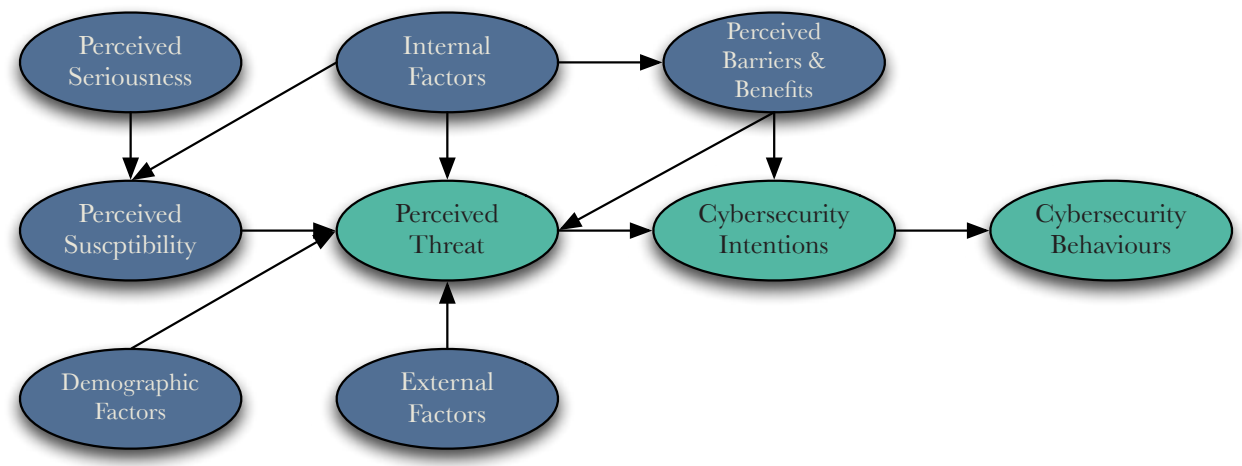

Figure 3-3. Conceptual model for examining home users' reasons for engaging in cybersecurity behaviours

that users' cybersecurity behaviours are influenced by their intention to engage in these behaviours. Cybersecurity intentions are expected to be influenced by users' perception of threat along with the perceived barriers to and benefits of engaging in these behaviours. The perceived threat is expected to be influenced by a number of internal and external factors along with the individual's perceptions of seriousness of and susceptibility to threats. This conceptual model was the starting point for approaching the research. However, it was expected that there could be additions and subtractions based on the empirical research. 
Table 3-1. Description of concepts and identification of components in the conceptual model

\begin{tabular}{|c|c|c|c|c|c|c|c|c|c|c|c|c|c|c|c|}
\hline 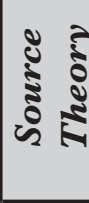 & & & 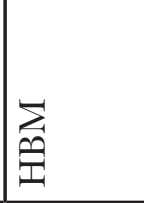 & $\begin{array}{l}E \\
ن \\
\end{array}$ & $\sum_{0}$ & 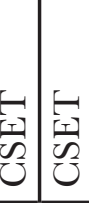 & 晃 & $\underset{\vdots}{\Xi}$ & $\sum_{0}$ & 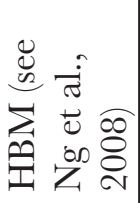 & 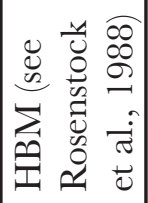 & $\underset{2}{=}$ & 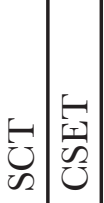 & 晃 & $\begin{array}{ll}2 & 0 \\
0 & 0 \\
0 & 0 \\
\end{array}$ \\
\hline 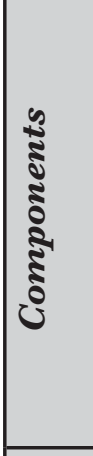 & & & 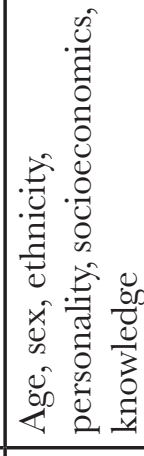 & 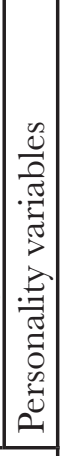 & 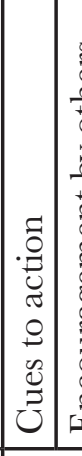 & 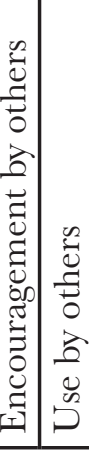 & 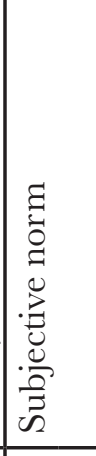 & 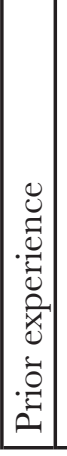 & 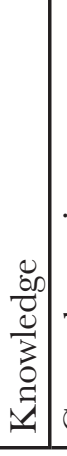 & 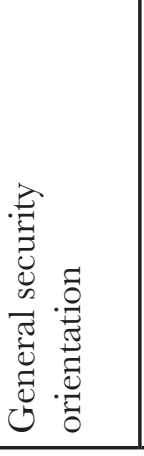 & 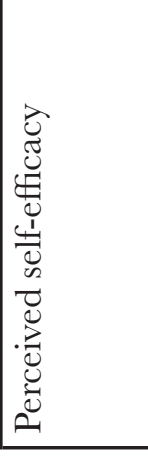 & & & 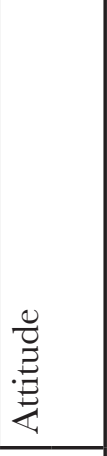 & 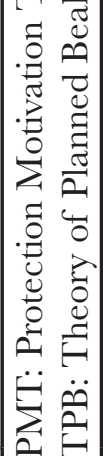 \\
\hline 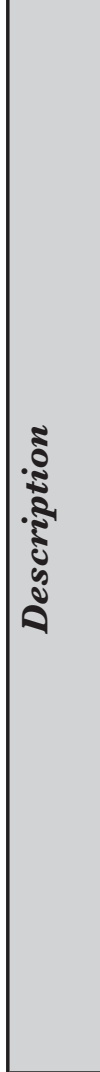 & 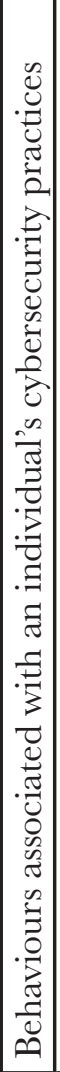 & 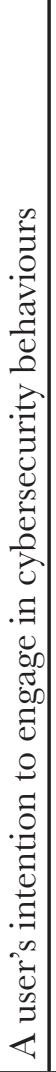 & 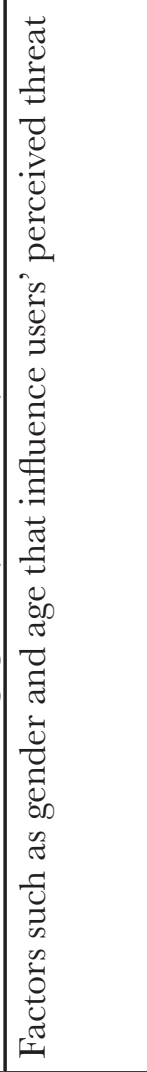 & & 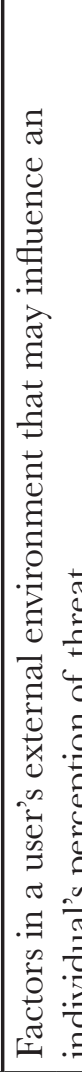 & 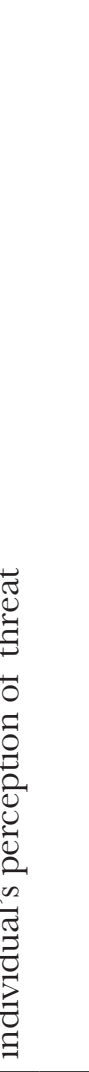 & 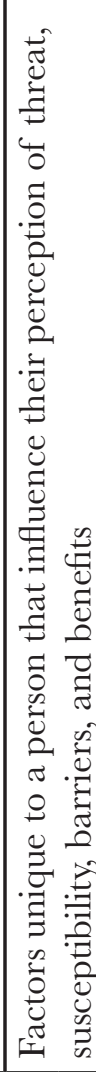 & & & & & & & & 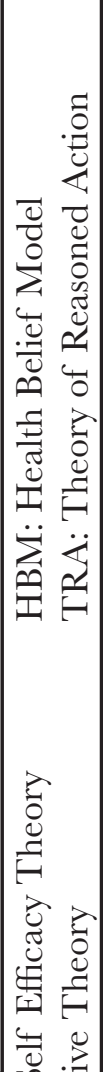 \\
\hline 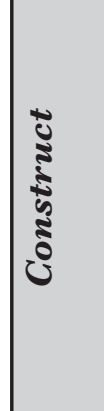 & 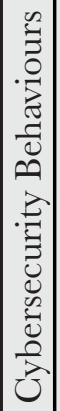 & 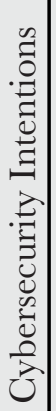 & 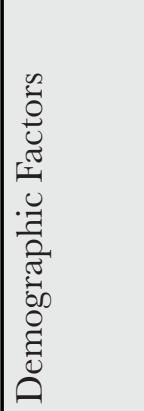 & & 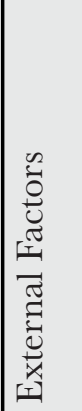 & & 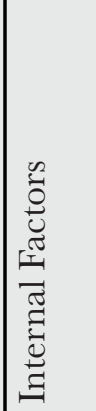 & & & & & & & & 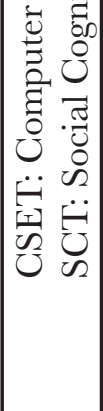 \\
\hline
\end{tabular}


Braun

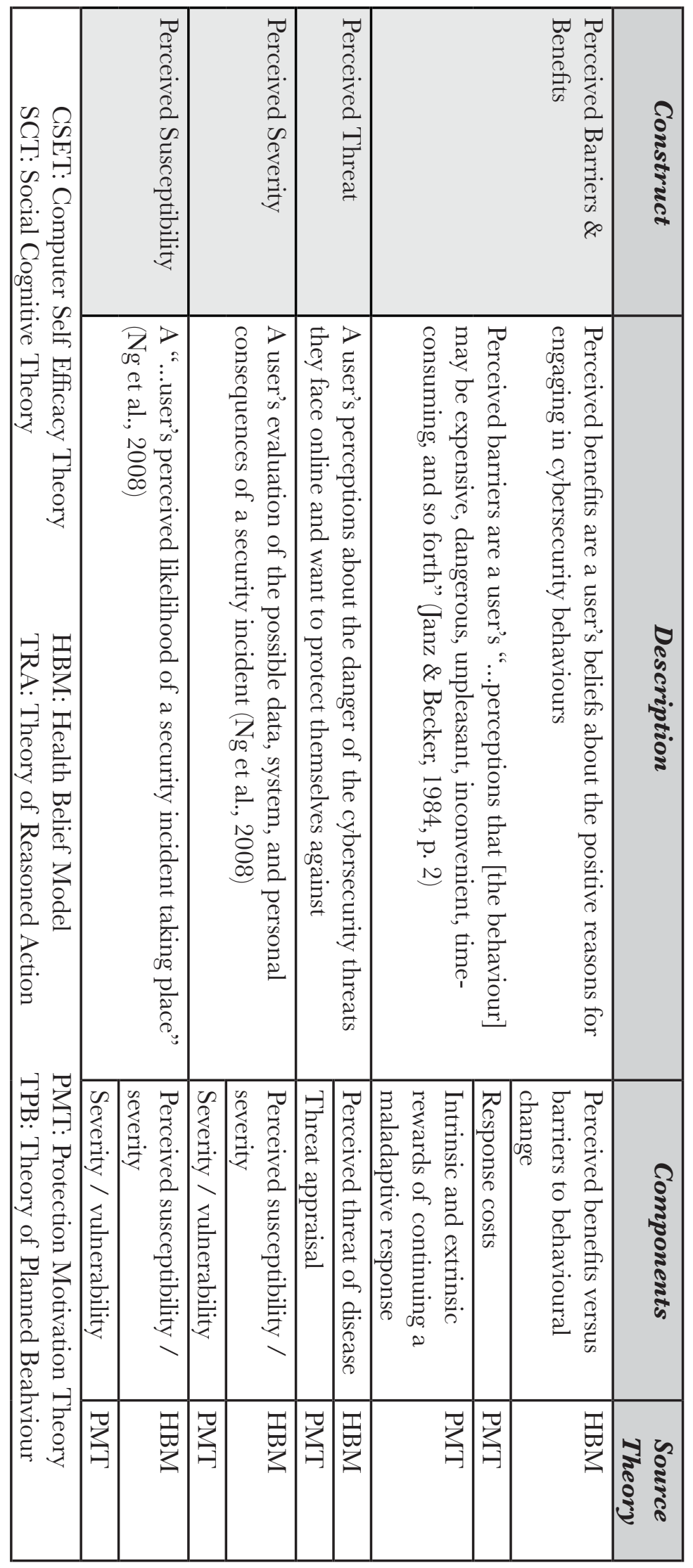




\subsection{Data Collection}

The data gathering was done in two stages. First, three focus groups were conducted. These were followed by 20 semi-structured individual interviews with individuals who had not participated in the focus groups. For the remainder of the thesis, the following terms are used to describe the respondents in the study:

- participants: respondents from the focus groups,

- interviewees: respondents from the individual interviews, and

- respondents: a combination of participants and interviewees, used to collectively describe the people who participated in the study.

In this section, an overview of the sample selection process and the respondents' characteristics is provided. This is followed by a discussion of the ethical considerations that needed to be addressed in this research. Next, details of the composition of and the data collection procedures for the focus groups and interviews are presented. Finally, steps taken to address rigour and trustworthiness are presented.

\subsubsection{Sample Selection}

Non-probabilistic sampling was used with the purpose of identifying "specific groups of people who either possess characteristics or live in circumstances relevant to the social phenomenon being studied" and who would "enable the exploration of a particular aspect of behaviour relevant to the research" (Mays \& Pope, 1995, p. 10). Respondents for the focus groups were approached based on their ability to meet the relevant criteria for the study. Both participants and interviewees were approached individually in person and asked to participate in a session if they met the required criteria.

Participants for the focus groups were approached based on different stages of their lives: part-time university students, working professionals, and older participants and retirees. These three groups provided a broad selection of respondents who covered distinct types of home users. This enabled data to be collected from individuals with different viewpoints based on how long participants had used computers, what they did on their computers, and their level of comfort with their computers.

A similar strategy was used for selecting interviewees. Initial interviewees were selected based on their ability to fulfil the criteria described in the next section. After 
the completion of the interview, they were asked if they knew other people who would be willing to participate in the study. The subsequent interviewees were approached to determine if they met the required characteristics and if they contributed to the breadth of interviewees selected.

\subsubsection{Ethical Considerations}

Ethical considerations needed to be taken into account before participants were interviewed. Gibbs (1997) identified several ethical considerations related to qualitative research:

- ensuring full disclosure about the purpose and use of participants' data,

- keeping participants well informed about the expectations of them, and

- handling sensitive material and confidentiality.

Each of these issues was addressed in respect to this research.

To ensure respondents were fully informed about the purpose and use of their data, information sheets were provided to all respondents prior to their participation in a focus group or individual interview session. The information sheet and interview questions for both data collection methods were approved by the Victoria University Human Ethics Committee prior to being given to the respondents. In addition, the participants signed a consent form (Appendix 5) allowing their data to be used in the research and the names of the respondents and any related organisations were changed in order to ensure anonymity.

In order to ensure that respondents were well informed about what was expected of them, a description of the research (Appendix 5) was given to them at the start of the session. The information sheet allowed each respondent to consider the issues before the focus group or interview took place. After the completion of all focus groups, a summary of the collected data was sent to participants. After each individual interview, the recording was transcribed and the transcription was sent to the interviewee to check.

The issue of handing sensitive material and ensuring confidentiality was managed differently between the focus groups and interviews. In the focus groups, participants were reminded that they were to keep the information they heard confidential. This was also made clear on the consent form that each participant signed before taking part in a focus group. For both focus group participants and interviewees, pseudonyms have been used in the thesis in order to maintain anonymity, and data were only disclosed to the parties 
indicated on the information sheet (e.g. the researcher, supervisors, and the respondent).

\subsubsection{Respondent Characteristics}

In order to qualify for being a respondent, four main criteria needed to be met. Each individual (1) needed to be a home computer user, (2) with a connection to the internet, (3) who was over 18 years of age, and (4) whose primary use of the computer at home was for personal use rather than working from home (e.g. telecommuting).

The definition of home computer users comes from Harrington et al. (2006) who defined them as "individuals conducting personal (including school-related) activities on a computer that is not owned or controlled by an organisation" (p. 1544). The purpose of limiting respondents to home computer users was to limit the influence of organisation mandated security behaviours as this was likely to change the influence of personal beliefs and reasons for engaging in security behaviours. Individuals who did not have a computer at home or who only had a computer in their home that was owned or managed by their employer did not qualify for this study. Individuals who used an organisation owned computer for part of the day and a home computer for another part of the day were asked to answer the interview questions in light of their home computing behaviours.

As the topic of this research required users to engage in online security behaviours, the presence of an internet connection on at least one of their home computer devices was required to qualify for the study. The type of internet connection (e.g. wired, wireless, mobile, etc.) did not disqualify respondents as long as one was present.

Although cybersecurity is an issue at any age, this research focused on adults (individuals age 18 or older) for several reasons. Adults are able to make purchasing decisions about hardware, software, and internet services. Therefore, it was assumed that they would be capable of making their own decisions about whether to engage in protective behaviours. There was an assumption that adults were more likely to be in a position where they could make decisions about what would constitute acceptable use of computers online. Finally, adults were assumed to be more likely to be aware of why they were making decisions about their behaviours and identify the reasons behind those behaviours.

The final requirement for respondents in this study was that the primary use of their computer was for personal use rather than work use. The reason for this was because work computers used in a home environment were likely to still have restrictions placed on their usage and could have still attracted organisational consequences if policies were breached. Therefore, in order for an individual to qualify as a respondent for this study they needed to own a personal computer used for personal reasons. A requisite amount of time that a computer was used for personal purposes was not set. However, it was expected that 
users would respond honestly about the main use of the computer. To further ensure that respondents were using a computer for personal purposes, they were also asked to describe their usual daily activities on the machine.

\subsubsection{Focus Groups}

The first two focus groups consisted of 5 participants and the final focus group consisted of 4. Each session lasted between 45 and 75 minutes and was recorded on video to help identify the speaker and to help capture nonverbal responses that may not have otherwise been captured in an audio-only recording. Participants were seated around a table with a whiteboard in front of them. Each session had three main areas of discussion: threats, safeguards, and behaviours. Participants were initially asked to describe how they used the internet on a regular day. This provided insight into how they generally used the internet which provided additional context for understanding what steps they took to protect themselves online. As a group, participants were then asked to help come up with a list of online security threats they were concerned about. These threats were written on the whiteboard for use as prompts in the subsequent questions about threats, threat awareness, and factors that led to their threat awareness. After discussing threats with participants, they were asked as a group to identify safeguards they were aware of to protect against threats online. The identification of these safeguards created a basis for respondents to discuss what safeguards they used, why they used those particular safeguards and what prompted that use. A final list of topics was created based on reasons why participants did use either the identified safeguards or other safeguards in order to protect their security.

At the end of the session, participants were given three sheets of paper on which they were asked to rank the top three threats they were concerned about, reasons for using safeguards, and barriers to using safeguards. They were asked to rank these items in descending order. Each person was asked to keep their responses anonymous, and the pieces of paper were returned in blank envelopes before the participants left the session. The responses listed were allocated points based on the ranking. The number one response for each of the lists was allocated 3 points, the second response 2 points, and the final response 1 point. The points from each of the topic lists were tallied for each group in order to identify the most important sub-topics within threat awareness, reasons for engaging in cybersecurity behaviours, and barriers to doing so. These rankings were used to compare the main topics of concern to those that were actually discussed and also to gain an understanding of which topics each group considered to be the most important.

Focus group 1 was comprised of part-time students who were studying at a university while working. As a result, the ages of the participants varied from 18-59 with the majority 
of participants falling into the 30-49 age bracket. The participants in this group were predominantly female (4/5). Most of the participants in this group had used the internet for at least 7 years. However, 2 participants were uncertain about how long they had been using the internet for. Every participant in this group indicated that they shared their computer with at least one other person.

Focus group 2 was comprised of respondents who were working full time. Respondents were in the 18-29 age bracket as well as the 50-69 age bracket. All of the respondents were working full time at the time of the focus groups. This group was the most evenly split between men (3/5) and women (2/5). Everyone in this group had used the internet for at least 10 years. Every participant in this group shared their computer with at least one other person.

Group 3 consisted of people who were primarily retired. Their ages were in the brackets of 40-49 and 50-69. The respondents in this group had worked in the past, but for the most part had left the work force at the time of the focus groups. The composition of this group was primarily female (3/4). One participant had been using the internet for less than 3 years while the remaining participants indicated that they had been using it for 10 or more years. In this group, half of the participants shared their computers while the other half did not.

While distinct types of individuals were selected in order to provide a variety of responses, this study is not meant to be generalisable. Despite differences in respondents' ages, the respondents in this study were adults living in a medium to large city who had generally had at least some tertiary education. Therefore, there is the potential to further explore the transferability of the results from this study to other populations (such as older or younger, individuals living in rural areas, individuals living in less developed areas, etc.).

\subsubsection{Individual Interviews}

The main purpose of the focus groups was to examine the suitability of the conceptual model in this study and to identify additional concepts not identified during the literature review. A set of 20 individual interview whose participants were distinct from focus group participants was conducted. The individual interviews were then used to build on the topics raised in the focus groups, gather more in-depth data on the identified topics, and add new topics when applicable. The questions asked in the individual interviews were informed from the responses given in the focus groups as well as from the literature and conceptual model. Each individual interview lasted between 45 and 90 minutes and the audio was recorded for transcription purposes. Interviews were conducted in sets of 5 so that preliminary analysis could be done in order to determine if additional areas needed 
to be explored based on interviewees' comments.

Interviewees were asked to describe the types of things they did online on an average day in order to provide some context for their computer use. Interviewees were also asked to define what they understood by the term "cybersecurity" to help ensure that when that term was used, an accurate interpretation was used in the context of each interviewee and to determine if interviewees had a similar understanding of the term. Each interview had three main areas of focus: threats, safeguards and enabling factors, and barriers. Interviewees were asked questions similar to those asked of focus group participants, with additional questions gained from discussions from the focus groups and previous interviews.

\subsection{Data Analysis Procedures}

All focus groups and interviews were recorded, then transcribed and coded using Nvivo 9. This section explains the data analysis procedures used for the focus groups and individual interviews. The first section describes the three stage iterative coding process used to analyse the focus group data. The next section explains the relationship between the focus group data and the individual interview data and describes the steps taken to analyse the individual interviews and how the codes from the individual interviews were reconciled with the codes from the focus groups.

\subsubsection{Focus Group Data Analysis}

The first stage of the coding process involved assigning preliminary codes to words and text segments based on their meaning and the context. The creation of the preliminary codes was initially guided by the conceptual model. However, the codes applied were not limited to those found in the model. Examples of codes found in the first stage of the coding process were:

- email fraud,

- phishing,

- spam,

- pornography,

- restricted Content,

- firewalls, and

- anti-virus. 
At this stage the primary focus was on ensuring that the relevant words and sections of text were appropriately identified. In order to ensure that the codes were applied consistently, descriptions for each code were included and used as part of the process to determine whether a section of text was relevant to the given code. This helped to ensure that the meaning of the codes was consistent.

In the next stage of the coding process, the codes that were identified in the first stage were grouped together based on their overall meaning or the relevant topic (see Table 3-2). At this stage, duplicate codes were checked to ensure that the meaning was the same. If the meaning was the same, the codes were consolidated. If different, Table 3-2. Example of how codes were the code was re-named to avoid confusion and the sections of text were re-checked to ensure that the codes were assigned appropriately. Because of the large number of codes that remained at the end of the second stage of coding, a third stage was undertaken where the grouped in the second stage of the focus group data analysis

\begin{tabular}{|l|l|}
\hline \multicolumn{1}{|c|}{ Topic } & \multicolumn{1}{c|}{ Code } \\
\hline Content Threats & Threat_Pornography \\
\cline { 2 - 2 } & Threat_RestrictedContent \\
\hline \multirow{2}{*}{ Email Threats } & Threat_EmailFraud \\
\cline { 2 - 2 } & Threat_Phishing \\
\cline { 2 - 2 } & Threat_Spam \\
\hline
\end{tabular}
focus was on further grouping and consolidating codes.

In the third stage, the codes were further grouped until the top level codes could not be combined into more general codes without losing meaning. At the highest level of codes, many of the assigned codes could be referred back to the components from the conceptual model. However, during the coding process, additional codes were also added to address categories that had not been included in the original conceptual model. An abbreviated example of the coding from this stage is provided in Table 3-3, p. 76. After the coding of the focus groups was complete, 27 top level codes remained (see Appendix $3)$. Although the original conceptual model only contained nine factors, the codes for the focus groups were not consolidated to such an extent at this point in order to minimise assumptions about which parts wold contribute to each factor. Therefore, although many of the original factors were included in the top level codes, there were also additional codes which represented components of the factors from the literature as well as additional factors identified during the focus groups. Throughout the process, preliminary indications of relationships between factors were noted in a separate document (see Appendix 4 for an example of a mind map used in the process of exploring the relationships between the factors). 
Table 3-3. Example of coding used in the third stage of coding the focus group data

\begin{tabular}{|c|c|c|c|c|}
\hline $\begin{array}{l}\text { Level } 1 \\
\text { Codes }\end{array}$ & $\begin{array}{l}\text { Level } 2 \\
\text { Codes }\end{array}$ & $\begin{array}{c}\text { Level } 3 \\
\text { Codes }\end{array}$ & $\begin{array}{l}\text { Level } 4 \\
\text { Codes }\end{array}$ & Description / Definition \\
\hline \multicolumn{4}{|l|}{ Threats } & Perceived security risks online and offline \\
\hline & \multicolumn{3}{|c|}{ Cybersecurity Threats } & $\begin{array}{l}\text { Perceived security threats that relate } \\
\text { specifically to online sources }\end{array}$ \\
\hline & & \multicolumn{2}{|c|}{ Content Threats } & $\begin{array}{l}\text { Perceived threats that focus on the } \\
\text { appropriateness of the material being } \\
\text { presented to users }\end{array}$ \\
\hline & & & \multicolumn{2}{|c|}{ Threat_Pornography } \\
\hline & & & \multicolumn{2}{|c|}{ Threat_RestrictedContent } \\
\hline & & \multicolumn{2}{|c|}{ Email Threats } & $\begin{array}{l}\text { Perceived threats that are transmitted via } \\
\text { email (either as an attachment or through } \\
\text { the message itself) }\end{array}$ \\
\hline & & & \multicolumn{2}{|c|}{ Threat_EmailFraud } \\
\hline & & & \multicolumn{2}{|c|}{ Threat_Phishing } \\
\hline & & & \multicolumn{2}{|c|}{ Threat_Spam } \\
\hline & & \multicolumn{2}{|c|}{ Malware Threats } & $\begin{array}{l}\text { Perceived threats that are found in } \\
\text { software created for malicious purposes }\end{array}$ \\
\hline & & & \multicolumn{2}{|c|}{ Threat_Virus } \\
\hline & & & \multicolumn{2}{|c|}{ Threat_TrojanHorse } \\
\hline & & & \multicolumn{2}{|c|}{$\ldots$} \\
\hline & & \multicolumn{2}{|c|}{ Other Threats } & $\begin{array}{l}\text { Perceived threats that do not fit into any } \\
\text { of the other categories or that would fit } \\
\text { into multiple categories }\end{array}$ \\
\hline & & & \multicolumn{2}{|c|}{ Threat_IDFraud } \\
\hline & & & \multicolumn{2}{|c|}{$\ldots$} \\
\hline & & \multicolumn{2}{|c|}{ Web Threats } & $\begin{array}{l}\text { Perceived threats that are transmitted to } \\
\text { users via websites }\end{array}$ \\
\hline & & & \multicolumn{2}{|c|}{ Threat_Cookies } \\
\hline & & & \multicolumn{2}{|c|}{$\ldots$} \\
\hline & \multicolumn{3}{|c|}{ Sources of Threats } & $\begin{array}{l}\text { Perceptions about how threats would be } \\
\text { transmitted to users' computers }\end{array}$ \\
\hline & & \multicolumn{3}{|c|}{ Source_Download } \\
\hline & & \multicolumn{3}{|l|}{$\ldots$} \\
\hline
\end{tabular}

\subsubsection{Individual Interview Data Analysis}

After the completion of the focus groups, a series of 20 semi-structured individual interviews was conducted. After the interviews were transcribed, the transcripts were returned to the respondents for checking. The goals of these interviews were to: (1) gain a deeper understanding of the topics identified in the literature review, and (2) to further 
explore the topics that were raised during the focus groups, and (3) identify any additional factors relevant to users' cybersecurity behaviours. The focus groups were intended to inform the individual interviews. Therefore, they covered many of the same topics. As a result of the focus groups, several new topics were explored in the individual interviews:

- perceptions about the characteristics of malicious users,

- the impact of luck on respondents' behaviours,

- the perception of accidents as a cause of cybersecurity breaches,

- the impact of respondents' reliance on others to protect them from potential breaches,

- perceptions about who is responsible for keeping a computer safe from a cybersecurity breach,

- the impact of past or present employment on respondents' behaviours,

- the impact of perceived data value on respondents' intention to protect their data, and

- the impact of computer sharing on respondents' behaviours.

These factors along with the other identified factors are discussed in greater detail in Chapter 4 .

An approach similar to what was used in coding the focus groups was used to code the individual interviews. The individual interviews were coded in a separate database from the focus groups in order to avoid overly influencing the coding process and overlooking new codes. However, the 27 top level codes from the focus groups were included in the individual interview coding database in order to provide some guidance.

Similar to the focus groups, the individual interviews were coded using an iterative process. Preliminary codes were assigned to words and text based on their meanings and these codes were subsequently categorised into higher level categories until once again the codes could not be further combined without losing meaning. 


\subsubsection{Integration of Focus Group and Individual Interview Data}

Once the interview and focus group transcripts were coded, the codes were merged into a single database in Nvivo, which resulted in duplicate and additional codes. In order to finalise the codes, a six step process was undertaken to check for duplicate, disparate, and analogous codes (see Table 3-4). The first step was to combine the codes which were identical for both the focus groups and individual interviews (situation 1, Table 3-4). In this case, both the name and description of the code were the same. When this happened, the same codes were kept and merged together into a single code in the combined database.

If there was a disparate code in the database where the name was the same but the definition of the code differed enough that the two codes were effectively different codes, the codes were reconsidered and the interview and focus group data were re-examined

Table 3-4. Steps taken to resolve duplicate and additional codes from the focus groups and individual interviews

\begin{tabular}{|l|l|l|l|}
\hline \multicolumn{1}{|c|}{ Situation } & \multicolumn{1}{|c|}{$\begin{array}{c}\text { Example Focus } \\
\text { Group Code }\end{array}$} & $\begin{array}{c}\text { Example } \\
\text { Intervieze Code }\end{array}$ & \multicolumn{1}{|c|}{ Resolution } \\
\hline $\begin{array}{l}\text { 1. Identical codes } \\
\text { (same name, same } \\
\text { meaning) }\end{array}$ & Perceived Severity & Perceived Severity & $\begin{array}{l}\text { Codes merged as Perceived } \\
\text { Severity }\end{array}$ \\
\hline $\begin{array}{l}\text { 2. Disparate codes } \\
\text { (same name, } \\
\text { different meaning) }\end{array}$ & $\begin{array}{l}\text { Future Threats } \\
\text { (meaning the } \\
\text { future likelihood } \\
\text { of threats) }\end{array}$ & $\begin{array}{l}\text { Future Threats } \\
\text { (meaning the } \\
\text { types of threats } \\
\text { respondents } \\
\text { thought they } \\
\text { would be likely to } \\
\text { face in the future }\end{array}$ & $\begin{array}{l}\text { Interview and focus group } \\
\text { data checked and assigned to } \\
\text { the appropriate code based } \\
\text { on the definition. Disparate } \\
\text { codes renamed based on } \\
\text { definition. }\end{array}$ \\
\hline $\begin{array}{l}\text { 3. Analogous codes } \\
\text { Future Threats }\end{array}$ & Future likelihood & $\begin{array}{l}\text { Codes merged and renamed } \\
\text { based on meaning (e.g. } \\
\text { Future likelihood) }\end{array}$ \\
\hline $\begin{array}{l}\text { 4. No disparate or } \\
\text { analogous code, } \\
\text { code fits within } \\
\text { higher level code }\end{array}$ & -- & $\begin{array}{l}\text { Code merged into the } \\
\text { appropriate higher level code } \\
\text { (e.g. Threats_WebThreats) } \\
\text { from the focus group }\end{array}$ \\
\hline $\begin{array}{l}\text { 5. No disparate or } \\
\text { analogous code, } \\
\text { no suitable higher } \\
\text { level group }\end{array}$ & -- & $\begin{array}{l}\text { Left to form a new top } \\
\text { level code pending a final } \\
\text { consolidation of codes }\end{array}$ \\
\hline $\begin{array}{l}\text { 6. Consolidation } \\
\text { of codes }\end{array}$ & -- & Best Practices & $\begin{array}{l}\text { All top level codes checked } \\
\text { to ensure they could not be } \\
\text { further grouped without } \\
\text { losing meaning. }\end{array}$ \\
\hline
\end{tabular}


(situation 2, Table 3-4, p. 78). Disparate codes were renamed based on their meanings. For example, when the focus group and individual interview codes were being combined, both sets had a code named "Future Threat". In the focus groups this was defined as the perceived likelihood that a person would have to deal with a threat in the future. In the individual interviews, this code was defined as the threats that a user was concerned they would have to face in the future. In order to resolve these differences, the transcripts were re-examined and the codes were checked to ensure that they matched the appropriate definitions. The final step in this situation was to rename the codes for clarity based on their meanings.

If a code did not have an identical or disparate code, the next step was to check if there was an analogous code where the name was different, but the meaning was the same (situation 3, Table 3-4, p. 78). In this situation, the codes were merged into a single code, using the code with the most appropriate meaning based on the definition. For example, the focus groups had a code named Future Threats while the interviews had a code called Future Likelihood. Both were meant to refer to the likelihood of an individual facing a cybersecurity threat in the future. Therefore, the code was renamed Future Likelihood, and the associated text segments were checked to ensure that they fit with the new code and associated meaning.

Where there were no identical, disparate, or analogous codes, the new codes were checked to determine if they could fit into one of the existing higher level codes (situation 4, Table 3-4, p. 78). If possible, codes were moved into existing higher level code groups. If it was not possible to move the code into a higher level group (situation 5, Table 3-4, p. 78), these codes were left as high level codes pending a final review of the codes for consolidation. As a follow up to this step, if a new code was established after the interviews, the focus group transcripts were re-examined to determine if there were any segments of text that could be assigned to the code. In the final step (situation 6, Table 3-4, p. 78), all codes were checked to see if they could be further refined or grouped together in a way that they would not lose meaning.

\subsection{Rigour and Trustworthiness}

In this final section, the issue of trustworthiness and rigour is discussed. The nature of interpretive research is that it is highly dependent on time and context, and can produce "divergent inquiry" (Lincoln \& Guba, 1985, p. 75). Therefore, interpretive research requires a different approach to examining reliability and validity than positivist research. Lincoln and Guba (1985) suggested four criteria for assessing the rigour and trustworthiness of interpretive research: credibility, transferability, dependability, and confirmability. These 
criteria replace internal validity, external validity, reliability, and objectivity (Lincoln \& Guba, 1985).

Credibility can be used in place of internal validity. Credibility can be achieved through prolonged engagement, persistent observation, triangulation, peer debriefing, negative case analysis, and member checks (Lincoln \& Guba, 1985). In this research, triangulation and member checks were used to increase credibility. Triangulation refers to the use of different sources and methods of data in order to cross-check findings (Lincoln \& Guba, 1985, p. 77). In this study, focus groups and interviews were used to cross check findings. Different sources were also used for the focus groups and interviews. Member checks relate to "soliciting reactions of respondents to the investigator's reconstruction" of the data (Lincoln \& Guba, 1985, p. 77). Focus group respondents were sent a summary of the overall findings from the focus groups and asked for their feedback on the initial conclusions that were drawn and the identification of topics that were of importance. The responses to the summary were positive and no topics were added or removed as a result of the feedback. Interviewees were sent copies of their transcript for checking. Also, at the completion of all of the interviews, interviewees were sent a summary of the results which they could comment on. This summary contained an initial set of revisions to the conceptual model that interviewees were asked for their feedback on. Initially the revised model started with a factor called "potential threat" which was comprised of threat awareness and consequence awareness. However, after discussions with several respondents it became clear that the focus needed to be on awareness, and a deeper understanding of threats and their consequences was better suited to exploring in conjunction with respondents' knowledge. After subsequent analysis and the identification of user types, five interviewees were approached separately, and had the user types, main characteristics, and behaviours explained to them. After the explanation, the interviewee was asked to identify which user type (if any), they felt best related to their cybersecurity behaviours. Four of the five interviewees identified themselves as the same type of user as they had been identified as during the analysis. The fifth interviewee did not identify the user type associated her as part of the analysis. However, this was expected, as explained in Chapter 6 (Dark Horse).

Transferability is similar to external validity. Transferability requires ensuring that the context of the research is clear so that other people can judge whether the findings can be applied elsewhere (Lincoln \& Guba, 1985). This has been done by providing the context of the research and describing relevant individual contextual factors that may have influenced respondents' answers.

In place of reliability, dependability can be used. Dependability can be achieved through the use of competent, external auditors (Lincoln \& Guba, 1985). To ensure dependability in this research, inter-rater validation was conducted in relation to the themes 
associated with the codes and the user types. Two senior academics were consulted and provided with a selection of transcripts in order to identify themes. The themes and codes identified by the academics were similar to those identified as part of the coding process in this research, supporting the interpretation of the data. Armstrong, Gosling, Weinman, and Marteau (1997) indicated that identifying similar themes, even if the specific codes within them are different, can provide support for the interpretation of the data. A similar process was undertaken with regard to the user types. The same academics were consulted with a selection of transcripts to identify which user types they felt were associated with each.

Confirmability can be used in place of objectivity and is primarily "concerned with the product (data and reconstructions) results" (Lincoln \& Guba, 1985, p. 77). Confirmability includes ensuring that an appropriate audit trail is created. The audit trail for this research includes the transcripts of the data, notes associated with the analysis, and notes associated with the process of conducting the revisions to the model and user types.

\subsection{Chapter Summary}

In this chapter, the use of the interpretive paradigm and qualitative approach to conduct exploratory research into home users' cybersecurity behaviours have been justified. As a starting point for the data collection, a conceptual model was developed based on the literature. In order to conduct the research, 3 focus groups and 20 individual interviews were conducted amongst home users. This chapter also provided an overview of how the data were analysed and how the codes between the focus groups and individual interviews were reconciled. In the next chapter, the findings from the data are discussed. 
Braun 


\section{Chapter 4}

\subsection{Introduction}

Chapter 4 explores the main findings with regard to research question one, answering "what are the factors influencing home users' cybersecurity behaviours?" In order to understand the context of the respondents' answers, their definitions of cybersecurity are compared to how researchers generally define the term.

In the next sections, the six main factors that emerged during the data analysis will be examined. These factors were:

- awareness,

- perceived danger,

- internal factors,

- external factors,

- barriers and enablers, and

- cybersecurity intentions.

Each of these factors and their relevant sub-factors are explored in greater detail in the subsequent sections.

As in the previous and remaining chapters, several terms are used to differentiate between the types of respondents. Participants is used to describe the respondents from the focus groups. Interviewees is used to describe respondents from the individual interviews. 
Respondents is used to describe the collective group of participants and interviewees.

\subsection{Understanding Cybersecurity}

During the focus groups, participants used the terms 'cybersecurity' and 'internet security' when talking about their protective behaviours. Throughout the discussions, these terms appeared to be used interchangeably. There was no indication during the focus groups that the terms were being used to differentiate different aspects of security. Therefore, the assumption was that participants understood the two terms to be identical and were using them interchangeably. In order to provide further context for interviewees' responses and to help interpret their answers, interviewees were asked to define cybersecurity. Along with providing additional context, asking interviewees for their definition of cybersecurity also helped to clarify how the interviewees defined the term since there is little consensus in the literature about what cybersecurity involves.

Two of the first three interviewees defined cybersecurity as being about "protecting their internet security". These vague definitions of cybersecurity suggested that some interviewees either viewed cybersecurity and internet security as distinct concepts or that they were uncertain about what cybersecurity involved which caused them to provide a vague definition. To further explore whether respondents viewed the terms differently or were uncertain about the meanings of each, subsequent interviewees were asked to define both cybersecurity and internet security to help clarify the meanings of these terms. Further interviewees were asked to define both terms. Thirty percent $(6 / 20)$ of interviewees considered cybersecurity and internet security to be identical and interchangeable. Interviewees' definitions were not corrected as part of the interviews, instead their definitions were used to ensure that the interpretations of their responses were accurate in the given context. Therefore, the focus was on obtaining a clearer idea of the context for the interpretation of their subsequent answers rather than ensuring that there was a single definition for cybersecurity.

No definitions of cybersecurity were identical. Despite differences, some common themes were present in the majority of interviewees' responses. Interviewees often focused on the threats that they felt they needed to protect themselves against. Viruses were commonly given as an example of something that interviewees needed to guard against. When references to the specific term 'viruses' were combined with mentions of other types of malware, these terms were seen in almost every interviewee's definition of cybersecurity. In their definitions, interviewees did not describe how threats would get to their computers. Interviewees also often noted that protecting their cybersecurity was about protecting their privacy. Although the definition of cybersecurity provided in Chapter 2 did not explicitly 
include privacy, it was noted that privacy can be protected through cybersecurity.

The remaining $70 \%$ of interviewees (14/20) considered cybersecurity and internet security to be distinct terms. The main way that interviewees distinguished cybersecurity from internet security was that internet security often included ways that users could protect themselves. Many of the definitions of internet security included tools and methods that could be used in taking protective steps. Definitions of cybersecurity and internet security both included protection of interviewees' privacy, personal information, and credit card numbers.

A summary of the main terms associated with definitions of cybersecurity and internet security is provided in Table 4-1. Although examining the terms used in defining cybersecurity and internet security did not immediately provide a revised definition, further examination showed how the terms can be distinguished from each other. Interviewees typically considered cybersecurity to be about the threats and risks faced online while internet security was about how users could protect themselves. Therefore, the definitions of cybersecurity and internet security for the remainder of this study have been revised as follows. Cybersecurity is defined as the protection of users from risks online (including, but not limited to malware, phishing, and social engineering) through the use of internet security. Internet security is defined as the tools and techniques that can be used by individuals to protect themselves from cybersecurity threats. The revision of these definitions has been done to better reflect users' understandings of each of the terms and was used in the coding process to ensure that these definitions were reflected accurately in the interpretation of each interviewees responses.

Table 4-1. Terms associated with interviewees' definitions of cybersecurity and internet security

\begin{tabular}{|c|c|}
\hline Cybersecurity & Internet Security \\
\hline $\begin{array}{ll}\text { - } & \text { electronic attacks } \\
\text { - } & \text { social engineering } \\
\text { - } & \text { cookies } \\
\text { - } & \text { popups } \\
\text { - } & \text { managing risk } \\
\end{array}$ & $\begin{array}{ll}\text { - } & \text { website security } \\
\text { - } & \text { hardware } \\
\text { - } & \text { network ports } \\
\text { - } & \text { firewalls } \\
\text { maintenance }\end{array}$ \\
\hline \multicolumn{2}{|c|}{$\begin{array}{c}\text { Terms Associated with Cybersecurity \& } \\
\text { Internet Security }\end{array}$} \\
\hline \multicolumn{2}{|c|}{$\begin{array}{l}\text { - } \quad \text { personal information } \\
\text { - }\end{array}$} \\
\hline
\end{tabular}

\subsection{Awareness of Threats and Safeguards}

Although security companies regularly put out reports about the most dangerous threats, this does not mean that all users are aware of which threats they need to be concerned about. The first step was to see what threats respondents were aware of. This provided the first insights into how knowledgeable about threats respondents were by listening to the way they described threats and the specificity of their descriptions. By 
getting respondents to brainstorm the threats, this gave them a starting point for comparing how they felt their awareness of threats compared to the normal user.

As discussed in section 3.4.4, within the focus groups, participants as a group compiled a list of the threats they were aware of and later ranked those threats in order of concern (see Table 4-2). Malware was consistently ranked in the top three threats. When participants discussed the types of malware they were aware of, viruses were mentioned in all of the focus groups. trojan horses and the more general term of malware were also frequently mentioned. Approximately one-third of participants were aware of trojan horses while only two participants discussed computer worms.

Table 4-2. Top ranked threats participants were concerned about, in descending order

\begin{tabular}{|c|l|l|l|}
\hline Rank & $\begin{array}{c}\text { Group 1 } \\
\text { Part-Time } \\
\text { Graduate Students }\end{array}$ & $\begin{array}{c}\text { Group 2 } \\
\text { Working } \\
\text { Professionals }\end{array}$ & $\begin{array}{c}\text { Group 3 } \\
\text { Retirees \& Older } \\
\text { Participants }\end{array}$ \\
\hline $\mathbf{1}$ & Malware & Malware & Financial Loss \\
\hline $\mathbf{2}$ & ID Theft & Malicious Websites & $\begin{array}{l}\text { Loss of } \\
\text { Confidentiality }\end{array}$ \\
\hline $\mathbf{3}$ & Phishing & Financial / ID Fraud & Malware \\
\hline
\end{tabular}

The top ranked concerns were most similar between the part-time graduate students and the working professionals. Along with malware, both groups were concerned about identity theft and fraud, as well as the potential for misleading or malicious content in the form of websites and emails. One reason for the similarity may have been because the participants in both groups were part of the workforce. Respondents in these groups were the most likely to indicate that the information they had received at work had contributed to their security awareness. In contrast, while the group comprised of retirees and older participants was concerned about similar threats, those threats were phrased in much broader terms and the consequences of the threats were put at the forefront. Older participants were often more concerned about financial loss and a loss of confidentiality. However, these "threats" were more closely aligned with the consequences of cybersecurity threats, rather than being threats themselves.

Although there was confusion between consequences of cybersecurity breaches and the causes of these consequences in all of the focus groups, it was most pronounced in the third focus group. Respondents were not corrected when they described consequences as threats. Their responses allowed for a better understanding and interpretation of whether the respondents were as aware of the threats as they considered themselves to be and whether their understanding of threats was accurate. Respondents were asked to clarify 
what they thought caused the consequences they had identified as threats. However, they did not always know, and at times emphasised that the consequences were what they were concerned about. Therefore, in order to provide an accurate overview of respondents' concerns, where appropriate the consequences have been included as threats that respondents were concerned about.

When comparing the threats identified by participants to those identified by interviewees, the results were similar (see Table 4-3). Along with identity theft or fraud as a concern, interviewees were similarly concerned about malware. Almost all interviewees noted at least one type of malware they were concerned about online, with a majority focusing on viruses as the greatest threat. The main area where interviewees differed from participants was in their awareness and concern about phishing. Approximately one-third of interviewees indicated a concern about phishing, while only 2 participants initially indicated a similar concern. The number of Table 4-3. Comparison of focus groups' top ranked people concerned about threats with interviewees' most frequently mentioned phishing in the focus threats groups increased slightly after the topic was raised by another participant. However, overall the number of participants concerned about phishing remained lower than it was

\begin{tabular}{|c|l|l|}
\hline Rank & $\begin{array}{c}\text { Aggregated Focus } \\
\text { Groups }\end{array}$ & Individual Interviews \\
\hline $\mathbf{1}$ & Malware & Viruses \\
\hline $\mathbf{2}$ & $\begin{array}{l}\text { Financial Fraud / Loss } \\
\text { ID Fraud / Theft }\end{array}$ & Identity Fraud / Theft \\
\hline $\mathbf{3}$ & $\begin{array}{l}\text { Loss of Confidentiality } \\
\text { Malicious Websites }\end{array}$ & $\begin{array}{l}\text { Other Malware (e.g. Trojan } \\
\text { Horses, spyware, etc.) }\end{array}$ \\
\hline $\mathbf{4}$ & Phishing & Phishing \\
\hline
\end{tabular}
for interviewees.

All of the focus groups and almost two-thirds of interviewees were concerned about identity theft or identity fraud. Respondents rarely brought up the causes of things like identity theft, financial loss, and other consequences and rarely linked specific threats and consequences together, even when prompted to discuss what they thought might cause the identified consequences. However, identity theft, much like financial loss, loss of confidentiality, unwanted access to individuals' computers, and data loss, can result from a cybersecurity breach caused by a malware, social engineering, or other type of attack. This sort of confusion about whether something is a cybersecurity threat or the consequence of one provides a starting point for further understanding respondents' comments about their awareness of threats as well as their understanding of them.

In order to provide additional context for what respondents' answers meant when describing how aware they were of threats, they were first asked to describe how aware of threats the normal user was. By getting the respondents to compare how aware they felt the normal user was, it provided insight into what they considered as the norm. A clear 
majority of respondents (24/34) believed that the "normal user" was reasonably aware of threats, primarily because the need for awareness was part of the internet culture:

\begin{abstract}
Several years ago [the normal user] probably wouldn't have been as aware [of threats]. But now it's like really ingrained in the culture really. You know what viruses are, and you know not to click on the email from the guy in Africa who is a prince and is going to give you $\$ 5000$ if you just give him your bank account number. (Par-1C)
\end{abstract}

Overall, the consensus among respondents was that the normal user was aware enough of threats to engage in basic protective steps, but lacked a thorough understanding of threats. This suggested that awareness and knowledge needed to be considered separately since some people considered themselves to be aware of the threats even when they did not understand them.

Respondents' levels of threat awareness varied greatly (see Table 4-4). Often respondents answered questions about their awareness, susceptibility, severity, and knowledge with initial responses where they considered themselves to be highly aware or unaware, for example. Where appropriate, such responses were assigned a code that indicated

Table 4-4. Respondent comments illustrating varying levels of cybersecurity threat awareness

\begin{tabular}{|c|l|}
\hline \multicolumn{2}{|c|}{ Threat Awareness } \\
\hline $\begin{array}{c}\text { Low } \\
(\mathrm{n}=4) \\
(\mathrm{n}=3)\end{array}$ & $\begin{array}{l}\text { "[I consider myself less aware } \\
\text { than the average user] just because } \\
\text { I have such a fear. And I don't } \\
\text { [pause] and if it happens, I don't } \\
\text { know what I'm going to do." (Par- } \\
3 D)\end{array}$ \\
\hline $\begin{array}{c}\text { Moderate } \\
(\mathrm{n}=5) \\
\left(\mathrm{n}^{\mathrm{i}}=8\right)\end{array}$ & $\begin{array}{l}\text { "I think I'm quite careful when it } \\
\text { comes to personal information on } \\
\text { the internet. So I think I'd be about } \\
\text { average, or maybe above average } \\
{[\text { in terms of how aware I am of }} \\
\text { threats]." (Par-1C) }\end{array}$ \\
\hline $\begin{array}{l}\text { High } \\
(\mathrm{n}=5) \\
\left(\mathrm{n}^{\mathrm{i}}=9\right)\end{array}$ & $\begin{array}{l}\text { "I have to clean off viruses from } \\
\text { PCs at work, so I know that people } \\
\text { aren't always as safe as they should } \\
\text { be. Or as careful as they should } \\
\text { be. You read about it on, you read } \\
\text { about articles, and you see it on the } \\
\text { news as well. Major virus attacks, } \\
\text { things like that, Trojans." (Int-6) }\end{array}$ \\
\hline
\end{tabular}

these levels. For a full explanation of how their responses were assigned to these categories, see Appendix 6 (p. 277). Respondents with lower levels of awareness were more likely to express a fear about the potential consequences of cybersecurity breaches and were more often unsure about the sort of steps to take compared to respondents with higher threat awareness. As users' confidence increased, so did their perceived awareness of threats. However, there were times where individuals' perceived threat awareness differed from the threat awareness they demonstrated. For example, when asked to describe how aware of threats respondents were, several indicated that they considered themselves as highly aware. However, throughout the 
sessions, these respondents talked about threats in very broad terms.

In contrast, other respondents considered themselves to have low levels of perceived awareness yet they demonstrated higher levels of actual awareness, often listing very specific and less common threats. In other cases, respondents considered themselves to be very aware of threats, yet only described these threats in the broadest of terms. Therefore, it became important to not only consider respondents' perceptions of awareness, but also the actual awareness that could be inferred from their responses.

Respondents' subjective assessment of their awareness of threats often provided insight into their confidence in their skills. However, their subjective beliefs that they were aware of the threats online did not always mean that they were as aware as they believed themselves to be. If respondents' awareness were solely assessed based on their subjective beliefs, it could lead to a misinterpretation of the role of awareness. As will be discussed in Chapter 7, despite many individuals considering themselves to be aware of threats, they still continue to act in unsafe ways. Therefore, awareness needs to be explored in terms of both perceived awareness, which can relate to perceived self-efficacy, and actual awareness which may be influenced by respondents' knowledge and other factors.

Although awareness of threats was important to understanding users' cybersecurity behaviours, on its own, it did not provide a complete explanation of what caused this awareness or what influenced intention to engage in safer behaviours. Just because individuals were aware of threats, it did not necessarily mean that they considered the threats to be dangerous nor did it mean that steps were being taken to protect themselves. Therefore, in conjunction with threat awareness, it was also necessary to look at the perceived danger of threats.

\subsection{Dangers of Threats}

Perceived danger refers to respondents' perceptions of how serious the identified threats were and how likely respondents felt they were to have to deal with such threats (perceived susceptibility). Throughout the remainder of the thesis, the term "threats" is used to collectively describe cybersecurity threats (e.g. malware, phishing, etc). The word "danger" is used to describe the possibility that individuals will be faced with these threats. Respondents' overall perceptions of danger were often lessened by their perceptions about the effectiveness of safeguards. Therefore, when respondents felt that the safeguards they were using were particularly good at preventing breaches, they often considered the overall danger to be lower. 


\subsubsection{Susceptibility to Threats}

Perceived susceptibility refers to beliefs about how likely an individual is to face a cybersecurity threat. Perceived susceptibility focuses on the likelihood of a security breach taking place in the future rather than in the past, though past events could influence perceived susceptibility. The impact of past experiences is discussed in section 4.5.1. Respondents were asked to describe how susceptible they felt the normal user was to cybersecurity threats. This created a baseline against which respondents' answers about their own perceived susceptibility could be assessed and interpreted.

The consensus amongst respondents was that they considered it possible that they would be faced with a security breach in the future (see Table 4-5). The majority of respondents (85\% of participants and $80 \%$ of interviewees) considered themselves to be moderately susceptible to a security breach. The two remaining participants fell on the low and high end of perceived susceptibility while the four remaining interviewees considered themselves to be highly susceptible to threats. Several reasons why respondents considered themselves to be more or less susceptible to threats were identified. These discussions were often focused on the effect of users' general characteristics, their perceptions about malicious users, and their perceptions of luck.

Table 4-5. Respondent comments illustrating varying levels of perceived susceptibility to cybersecurity threats

\begin{tabular}{|c|c|}
\hline \multicolumn{2}{|r|}{ Perceived Susceptibility } \\
\hline $\begin{array}{l}\text { Low } \\
\left(\mathrm{n}^{\mathrm{f}}=1\right) \\
\left(\mathrm{n}^{\mathrm{i}}=0\right)\end{array}$ & $\begin{array}{l}\text { "PPatches and updates from software companies] are recent enough. And } \\
\text { [malicious users] would probably attack the States first and then any fixes } \\
\text { will be done overnight. And then I'll get them in the morning, [limiting my } \\
\text { susceptibility]" (Int-17) }\end{array}$ \\
\hline \multirow[t]{2}{*}{$\begin{array}{l}\text { Moderate } \\
\left(\mathrm{n}^{\mathrm{f}}=12\right) \\
\left(\mathrm{n}^{\mathrm{i}}=16\right)\end{array}$} & $\begin{array}{l}\text { "I suppose the threat [in the future] won't really be any less than what it is now. } \\
\text { I don't know if it's the same or more, but there are certainly people out there } \\
\text { that are trying to capitalise, and you know, steal. Just like somebody robbing a } \\
\text { bank." (Par-3A) }\end{array}$ \\
\hline & $\begin{array}{l}\text { "I'd like to say that I hope not likely. But there is a chance that I could have to } \\
\text { deal with it, yeah. Simply because of the proliferation of the software out there. } \\
\text { And you never know what they're going to come out with next." (Int-3) }\end{array}$ \\
\hline $\begin{array}{c}\text { High } \\
\left(\mathrm{n}^{\mathrm{f}}=1\right) \\
\left(\mathrm{n}^{\mathrm{i}}=4\right)\end{array}$ & $\begin{array}{l}\text { "I think the odds are quite good because surfing the net, you're always picking } \\
\text { up viruses even though you have good security software. It's always detecting } \\
\text { something... And if you happen to have let your security license lapse by a } \\
\text { couple of days, you can actually really get hit quite bad." (Par-1E) }\end{array}$ \\
\hline
\end{tabular}

Respondents often felt they were less susceptible to threats because they did not fit into the categories of people they believed malicious users would target. The most 
commonly mentioned targets were:

- people with valuable data,

- Windows PG users,

- rich people,

- businesses,

- governments, and

- other public / private institutions (e.g. universities, NGOs, etc.).

Respondents were not specifically asked to identify groups of people they considered to be more susceptible to threats. When describing their own susceptibility to threats, they often identified these targets as a way to describe who they considered more susceptible than themselves. The implication was that they did not need to be concerned about their security because they were not attractive targets. In order to further understand why these particular categories of people were considered more susceptible to threats it is necessary to explore what it is that distinguishes these groups from the respondents. These distinguishing features can be regarded in light of security by obscurity, data value, and assumptions about malicious users.

Security by obscurity ${ }^{1}$ can be used to describe why individuals who used Mac operating systems considered themselves to be at a lesser risk than their Windows counterparts. Although only a minority of respondents (3/14 participants and 3/20 interviewees) used the Mac operating system, there was a common belief among them that their choice of operating system lessened their susceptibility to threats:

- We're not so worried about viruses [in my household], but maybe that's just, maybe I'm being stupid. But Macs don't get viruses. (Par-1B)

- I generally have, keep my viruses definitions up to date. I also use a Mac which has very few, well, isn't targeted by malicious software at the moment. (Int-15)

In this situation respondents were making the assumption that the relatively low market share of Apple computers contributed to their security since many considered it to be "not profitable" for malicious users to target a small community of users.

Although the idea that certain types of computers were less vulnerable was unique

1 The idea of security by obscurity or security by minority is that the less well known an individual / organisation / system is, the less likely it is to be targeted. This idea has been extended to the computer security realm with exploring open source software (see Swire, 2004) and Mac computers due to their traditionally low market share compared to Windows PCs (see Kotadia, 2005) 
to Mac users, the idea that respondents did not have valuable data was much more widespread. Respondents often believed that if they did not possess "valuable data," they were less susceptible to threats:

- I'm not an important target... I don't have anything valuable [on my computer] (Int-2)

- [1] have a dumb machine that lets me in and doesn't keep anything of mine. If I have anything [of value], I take it off and put it on a new PC or on a flash card, and that's where I keep it. (Par-2E)

Value related to the perceived value of the information that was held on respondents' computers (e.g. sensitive documents) or the value in using that information (e.g. credit card numbers stored on a user's computer). When discussing the value of data on a personal computer, respondents often explained that they believed that other groups of people who held more valuable data, such as organisations and government bodies, were more susceptible to cybersecurity breaches than they were themselves.

Governments, businesses, and other large institutions were often identified as being better targets for malicious users. Respondents believed that these organisations not only held more data, but that these data were more valuable than what was contained on a personal computer. Therefore, these organisations were considered to be more susceptible to threats:

It's much more likely that they'll hack into [my bank]... it's just not viable from their standpoint for all the effort they go through when they could easily hit a large database that has names, dates of birth, etc. (Par-2A)

This comment not only suggested that the main goal for malicious users was to get data, but also that organisations and home users were essentially on equal footing for security where the choice for malicious users was between selecting one target with a multitude of data or multiple targets with minimal data. Comments about the value of organisational data as well as data kept on users' home computers implied that respondents made assumptions that getting access to data was malicious users' main objective.

Respondents' comments about value judgments illustrated how users' beliefs about malicious users influenced their perceptions about susceptibility to and severity of threats. Throughout the interviews and focus groups comments were made about malicious users' reasons for engaging in malicious behaviour and the expected outcomes (see Table 4-6, p. 93). In particular, the expectation that malicious users sought to defraud specific victims for financial or information gain further illustrated respondents' beliefs that the end goal 
Table 4-6. Respondent descriptions of malicious users, reasons for malicious users to engage in malicious behaviours, and the outcomes malicious users' hoped to accomplish

\begin{tabular}{|c|c|c|}
\hline $\begin{array}{c}\text { Descriptions of Malicious } \\
\text { Users }\end{array}$ & $\begin{array}{c}\text { Reasons for Engaging in } \\
\text { Malicious Behaviours }\end{array}$ & $\begin{array}{l}\text { Expected Outcomes of } \\
\text { Malicious Behaviours }\end{array}$ \\
\hline 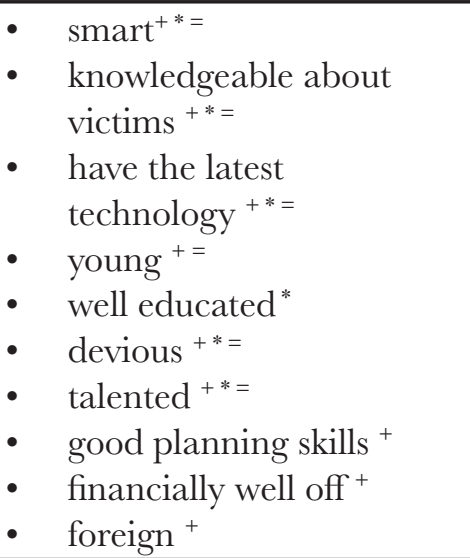 & $\begin{array}{ll}\text { - } & \text { show cleverness } \\
\text { - } & \text { nothing to lose } \\
\text { - } & \text { prank people }^{+} \\
\text {- } & \text { defraud victims } \\
\end{array}$ & $\begin{array}{ll}\text { - } & \text { prove skill }{ }^{+=} \\
\text {- } & \text { financial gain }{ }^{+*}= \\
& \text { information gain } \\
\end{array}$ \\
\hline \multicolumn{3}{|c|}{$\begin{array}{ll}+ & \text { identified during focus groups } \\
* & \text { identified during individual interviews } \\
= & \text { identified by multiple respondents }\end{array}$} \\
\hline
\end{tabular}

for malicious users was to obtain data from users rather than to use malware to harvest computing power to conduct distributed denial of service (DDoS) attacks or to distribute spam and other malware.

Respondents typically had a positive view of malicious users (see Table 4-6). For instance, that malicious users were considered to be smart, talented, well educated, and financially well off, all of which suggested a positive view of these individuals. These beliefs about malicious users provided respondents with a way to imply that if they were susceptible to threats, it was not necessarily their fault since the malicious users were smarter or more talented than they were. Further, this appeared to take some of the culpability off the respondents because of their assumption that malicious users were able to get around the safeguards that respondents took.

Although perceptions of value and expectations about malicious users influenced respondents' perceived susceptibility, perceived susceptibility was also influenced by perceptions of luck. Luck was typically associated with the likelihood of a breach. Some respondents felt that so far, they had either been lucky enough not to have been attacked or that they had been unlucky enough to have suffered a breach. A comment from a participant summed up many of the comments about luck when she stated:

I figure it's just [pause], it'd be a fluke. It would be bad luck if somebody infiltrated our computer. (Par-2E)

Similar comments about luck were made throughout the focus groups. This resulted in the concept of luck being explored in greater detail during the individual interviews. 
Comments about luck often had an underlying sense of resignation about respondents' susceptibility to threats because of the implication that there was nothing that could be done to avoid threats if luck was not on their side. Comments about luck also had an underlying sense of fatalism with statements that blamed "bad luck" for causing security breaches. In particular, luck in relation to susceptibility was referred to more often when respondents considered themselves to be less knowledgeable about cybersecurity. The relationship between knowledge and luck is described in greater detail in section 4.5.2.

\subsubsection{Severity of Consequences}

Respondents based their judgments about the severity of a threat on several factors: (1) the threat itself, (2) the perceived goals of malicious users, (3) perceived data value, (4) the perceived effectiveness of safeguards, and (5) knowledge of threats and safeguards. The effect of the threat itself, perceptions about the value of data, and the perceived goals of malicious users is discussed in this section. The perceived efficacy of safeguards and its relation to perceived severity is discussed in section 4.4.3. Knowledge of threats and safeguards is discussed in section 4.5.2.

Certain threats, such as viruses, were deemed less important because the consequences of these threats were not considered to be as severe as other threats. The lack of concern over the consequences of certain threats stemmed partially from uncertainty about the potential consequences of these threats. Two participants discussed their beliefs about the intended purpose of viruses:

With viruses anyway, is it just pranking you or something? Like are they trying to get something from you when they send out a virus, or? (Par-1C)

[They are] trying to be clever I think. (Par-1A)

Comments such as these demonstrate an unfamiliarity with the consequences of viruses and other types of malware. Respondents often illustrated a lack of understanding about the consequences of threats by downplaying their severity. This was highlighted by respondents associating non-malicious consequences with threats.

Similarly to how respondents assumed that the consequences of breaches were nonmalicious, they also considered the goals of malicious users to be more benign. Throughout the focus groups and interviews, respondents commented on what they believed were malicious users' reasons for engaging in malicious activities and their goals with the outcomes (see Table 4-6, p. 93). Respondents often believed that malicious users simply wanted to show how intelligent they were, either to other malicious users or to their intended victims. 
Respondents' underlying assumptions that malicious users' intentions were benign their beliefs that the consequences of breaches were less severe. Respondents who considered the intentions of malicious users' to be benign typically did not believe that they would suffer consequences greater than being inconvenienced. While respondents understood that malicious users could have more malicious goals such as financial and information gains, they typically did not consider these to be severe consequences that they needed to worry about themselves. Respondents often felt that their data was either not particularly valuable which meant it would not be targeted or that they were not one of the main types of targets that malicious users' preferred to attack.

Using the same method as was used for exploring perceived susceptibility, respondents were first asked to describe how severe the normal user would consider the consequences of a cybersecurity breach to be. After this, they were asked to consider how severe the consequences would be for themselves. Most respondents considered the consequences of threats to be severe. However, some felt the threat was mild because of the type of content kept on their computers. Few considered the severity to be moderate (see Table 4-7). The majority of respondents were typically either unconcerned about data loss, corruption, or inability to access data as a result of a cybersecurity breach or very concerned about it.

Table 4-7. Respondent comments illustrating varying levels of perceived severity of consequences of cybersecurity threats

\begin{tabular}{|c|c|}
\hline \multicolumn{2}{|r|}{ Perceived Severity } \\
\hline \multirow[t]{2}{*}{$\begin{array}{l}\text { Lowe } \\
\left(\mathrm{n}^{\mathrm{f}}=4\right) \\
\left(\mathrm{n}^{\mathrm{i}}=10\right)\end{array}$} & $\begin{array}{l}\text { "I don't have any movies or videos or songs on mine, and I figure I could } \\
\text { reconstruct pictures. Everybody else could email me stuff. I don't see it as } \\
\text { that big of a deal." (Int-19) }\end{array}$ \\
\hline & $\begin{array}{l}\text { "Most home data I couldn't give a stuff about actually... I don't really have a } \\
\text { lot of home data that I would care very much about." (Int-5) }\end{array}$ \\
\hline $\begin{array}{c}\begin{array}{c}\text { Moderate } \\
\left(\mathrm{n}^{\mathrm{f}}=1\right) \\
\left(\mathrm{n}^{\mathrm{i}}=3\right)\end{array} \\
\end{array}$ & $\begin{array}{l}\text { "...somebody stealing my financial information would be a pretty big deal. If } \\
\text { it were just a minor virus that somebody put through, that's easily taken care } \\
\text { of. I'm not that worried." (Int-3) }\end{array}$ \\
\hline \multirow[t]{2}{*}{$\begin{array}{l}\text { High } \\
\left(\mathrm{n}^{\mathrm{f}}=9\right) \\
\left(\mathrm{n}^{\mathrm{i}}=7\right)\end{array}$} & $\begin{array}{l}\text { "We always save a lot of movies and photos in the computer... So if I lost } \\
\text { that data I would be very sad because all those records, moments, and } \\
\text { experiences with my son who is so very precious to me would be lost." (Int-1) }\end{array}$ \\
\hline & $\begin{array}{l}\text { "I have tens of thousands of photos, and I keep those on my computer... } \\
\text { if something were to happen that would corrupt those? I would be pretty } \\
\text { shattered... as a photographer, they're my art. They're things that I've } \\
\text { worked on, that I've created, that I've made into something. And losing that } \\
\text { would be pretty devastating." (Int-7) }\end{array}$ \\
\hline
\end{tabular}

One of the few areas where age appeared to play a role was with respondents' perceptions of severity. Respondents with the lowest perceptions of the severity of the 
consequences were in their mid-fifties or older. As the age of the respondent decreased, their concern about the consequences increased. Younger respondents tended to store more digital media (such as photos, videos, and music) on their computers. This digital media represented either a financial or emotional investment. Even respondents who were concerned about the consequences typically indicated that these concerns were limited to data they considered to be valuable rather than it being an overall concern about all data on their computer. The other main area where age appeared to influence respondents' behaviours was reliance, which is discussed in section 4.5.4.

The relation of data value to threat severity first emerged during the focus groups when several participants made passing comments about how they considered the severity of threats to be low because they did not have any valuable data. However, there was little agreement on which types of data were most valuable. Some of the types of data considered to be most valuable were:

- personal photographs,

- home movies,

- purchased music and movies,

- work or school related files, and

- personal information (e.g. letters, emails, addresses).

The value of data often depended on what was most important in the respondent's life at the time. Students or people who brought work home were most concerned about their school or work files and considered this to be valuable data. These users could be considered organisational users by day and home users by night because the majority of their computer use at home was for personal reasons. In contrast, parents of young children were often more concerned about their personal photos or movies which in most cases took the form of digital media stored on the computer.

Perceived value of data was explored in greater detail during the individual interviews in order to gain a greater understanding of what made the data valuable. The ability to replace the data if something happened where it could no longer be accessed often helped respondents determine its value. As noted in Table 4-7, p. 95 (low severity), respondents who felt that they could replace or replicate data often considered the consequences to be less severe, whereas those who felt the data could not be replicated considered the consequences of losing access to the data to be more severe. However, respondents' who based their perceptions of severity on their ability to replicate or reproduce data often of value did not take into consideration what would happen if the data were disclosed rather than made inaccessible.

Data disclosed in a privacy breach was often associated with the disclosure of financial 
information (e.g. credit card or bank account numbers) or with personal information that could lead to identity theft. Respondents indicated that these types of breaches would limit their ability to participate normally online. Half of the participants and 35 percent of interviewees noted that a cybersecurity breach would change how they used the internet. Several participants in the third focus group discussed how a cybersecurity breach would change how they used the internet:

We'd become very distrustful [if we experienced a cybersecurity breach]... I would probably not use my credit card again online. (Par-3A)

[I would] shut down everything. I wouldn't bank online any longer. I would stop that. I wouldn't shop again [online] either. (Par-3B)

Or I'd look into some software that would give you some protection. (Par-3A)

Go back to cash (Par-3B)

Yeah. (Par-3C)

Similar statements were echoed by interviewees. One described what she was most concerned about regarding a potential breach and the severity of the consequences:

I think the thing I'm most concerned about is someone destroying my sense of security. I would, [pause], I think it would be really difficult for me to recover in terms of a person and to be trusting online... It [a breach] would limit my capacity to perform normally. (Int-18)

When described in terms such as the response above, the threat of a cybersecurity breach can be likened to the result of a personal assault.

How men and women described the severity of the consequences cybersecurity breaches differed. Several female respondents had similar reactions to the response above. They noted that a cybersecurity breach would essentially cause them to stop using the internet due to the violation of their sense of security. In this way, the computer became an extension of the individual. In contrast, of the men who indicated that a cybersecurity breach would change their behaviours, none described it in such strong personal terms. Instead, there was a clearer distinction between what affected their computers versus what affected them as individuals, for example:

My computer might be targeted as a drone, which just hurts my computer. It doesn't really hurt me. (Par-2A) 
Similar responses were much more commonly made by male respondents who typically indicated that a breach would make them more cautious, but would not stop them from using the computer.

Although there was a divide between how men and women viewed the severity of a cybersecurity breach on an emotional level, the distinguishing feature may also relate to how individuals viewed the computer in relation to themselves (e.g. as an extension of themselves or as a distinct entity) rather than based entirely on gender. In cases where the cybersecurity breach referred to data disclosure rather than data accessibility, the value was not associated with the data itself, but rather with the value of the feelings of security. These feelings of security were much more difficult to assess due to the individual nature of it. Overall, it appeared that in many cases respondents preferred to downplay the potential severity of the consequences based on what they considered as the value of the data.

\subsubsection{Effectiveness of Safeguards}

Most participants and interviewees believed that having protective software, such as anti-virus software, would lessen the impact of threats online. This appeared to be because the protective software would either: (1) prevent at least some or all of the consequences or (2) provide an additional feeling of security because there was an expectation that the security company would remedy any breach that occurred.

The topic of safeguard efficacy emerged during the first focus group discussion when several of the participants noted that they felt that the safeguards they used were enough to protect them from the various threats they might encounter online. The safeguards identified during the focus group were predominantly protective software rather than protective behaviours. During the remaining focus groups, the effectiveness of safeguards frequently emerged when participants spoke of how likely they felt they were to suffer a cybersecurity breach.

Respondents' beliefs about their susceptibility to threats and the severity of the consequences were further influenced by how effective respondents believed their safeguards were. They frequently indicated that the use of protective software would either prevent the breach from occurring or it would minimise and / or remedy the consequences of a breach. Respondents often considered the safeguards that they used to be effective at 
preventing breaches, for example:

I keep my virus software up to date [so I'm not very concerned about threats]... If the software wasn't picking it up and it happened reasonably regularly, [the security companies] wouldn't be in business. [The security companies] have got too much money invested in their software to be too far behind the eight-ball. (Int-6)

Comments such as the one above indicated a relatively high level of trust in the companies that made effective protective software. However, not everyone was as confident. One of the participants questioned whether trust in safeguards was deserved:

We have these software, you know, protection. And you know, whether that be Norton or whatever, and we think that it's all being taken care of for us, when in reality it's probably not. (Par-3C)

Overall, she considered her safeguards to be effective at preventing threats. However, there appeared to be a sense of concern in her further comments that she did not necessarily know enough about security to accurately judge whether the safeguards were as effective as she hoped. The general belief amongst the respondents was that protective software was effective at preventing threats and mitigating the consequences.

Most respondents (12/14 participants and 20/20 interviewees) considered their safeguards to be at least moderately effective. Three interviewees considered their safeguards to be moderately effective and surprisingly, 17 interviewees considered them to be highly effective. In contrast, 12/14 participants considered their safeguards to be moderately effective and one to be highly effective. One possible explanation for why more interviewees than participants considered their safeguards to be highly effective is because participants may have felt that if they overstated the effectiveness of their safeguards another participant would challenge their opinion.

The general consensus appeared to be that protective software offered the greatest benefit of all the possible safeguards that could be used. The most commonly used types of protective software were antivirus software and firewalls. One of interviewees commented on how much protection he felt his firewall offered:

I installed a firewall and I also have the program that monitors the activity, etc. So I think that should provide at least $70 \%$ of the safeguards against the threat. So the remaining 30\% sometimes you just have to deal with the uncertainty. (Int-4)

Passwords were considered to be less effective at protecting from threats. A participant 
described the level of protection he felt that his password offered him:

Other than the firewalls, I don't think the passwords are doing that much [to protect me online]. (Par-2D)

He went on to explain that he had lost his passwords several times and had them reset due to security breaches on websites. Although other respondents and interviewees considered passwords to be potentially weak, none indicated that they used multiple forms of authentication on websites.

Respondents were often aware of some behavioural safeguards that they could undertake and many seemed to understand that there was some benefit in doing so. As an interviewee described:

You can reduce your chances by being careful though. Just be aware of what you're downloading and what you, you know $[\mathrm{do}]$. (Int-20)

Safe behaviours typically consisted of not opening email attachments from unknown sources and not visiting unknown websites. Few respondents (3/14 participants, 6/20 interviewees) knew of more involved methods for protecting themselves, such as disabling plugins, checking recent computer activity, and disconnecting from the internet. Respondents who took these steps were in many cases more likely to consider their safeguards to be only moderately effective. In addition, they tended to be more concerned about security overall, which suggested that increased levels of knowledge decreased respondents' beliefs in the effectiveness of safeguards.

In many cases, it appeared that participants and interviewees believed that their safeguards offered almost complete protection from threats. Even the lone participant who did not use safeguards stated:

I'm certain there are lots of things that are out there that offer almost complete protection against any perceivable threat. (Par-3A)

This belief in the effectiveness of safeguards allowed people to essentially dismiss much of the potential danger of threats since they believed that protective software would prevent a breach in the first instance and if there were a breach, the software company would remedy it. These beliefs combined with beliefs about the motivations and goals of malicious users allows people to downplay the potential danger of security threats and to distance themselves from the need to be concerned. 


\subsubsection{Summary of Threat Danger}

Overall, respondents felt that it was at least somewhat likely that they would have to face cybersecurity threats in the future. Factors that they felt made them more susceptible to threats included having data and files that were valuable on their computers and being "rich." Because many respondents did not consider themselves to fall into either of these categories, they tended to assume that if they did have to face a cybersecurity threat, it would be more of a "fluke." The severity of the consequences of threats often depended on how much value respondents placed on the data that they had. If they considered their data to be difficult to replace or of personal value, they considered the severity of the potential consequences to be greater. However, if they believed that they could easily replicate the data, the perceived consequences were often less severe. Furthermore, the severity of the consequences also depended on how respondents viewed malicious users. Respondents who viewed malicious users' actions as being more benign tended to believe the consequences would be less severe than respondents who believed that malicious users were acting in truly malicious ways. Beliefs about the effectiveness of safeguards affected perceptions about the likelihood of having to face cybersecurity threats and the severity of the consequences. There was often an assumption that protective software could help minimise the risk of having to face threats in the first place. Furthermore, respondents often assumed that the people responsible for making the protective software would help to ensure that their systems would be returned to normal if it were breached. These perceptions of danger were further influenced by factors internal and external to the respondents.

\subsection{Influential Internal Factors}

During the focus groups and interviews, seven internal factors were identified as being relevant to users' security behaviours (see Figure 4-1). Prior experience was associated with the feelings about the consequences associated with actual or perceived cybersecurity breaches. The ability to identify a cybersecurity breach often depended on an individuals' knowledge. Knowledge

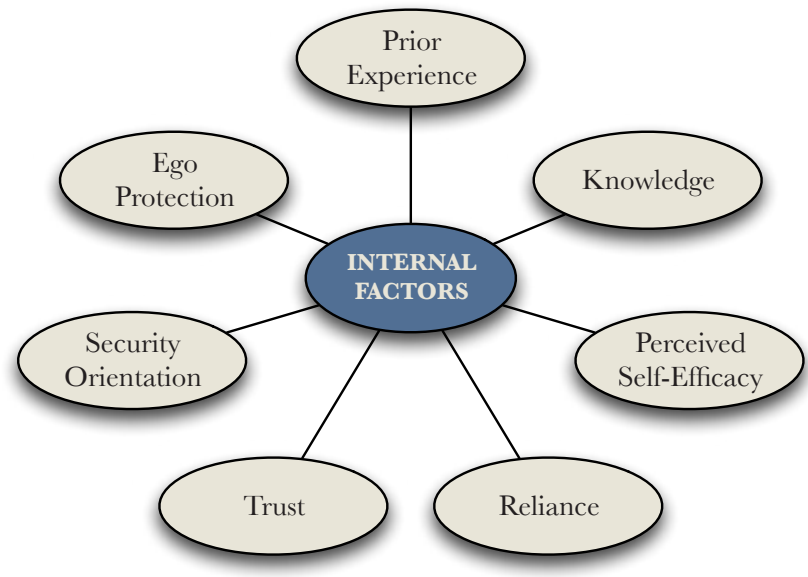

Figure 4-1. Internal factors that influenced home users' cybersecurity intentions 
was associated with respondents' understanding of the threats and safeguards. Perceived self-efficacy referred to respondents' confidence in their ability to protect themselves online. Reliance was focused on how much respondents depended on others to protect them from threats and to remedy breaches if they occurred. Trust was used to describe the confidence that respondents had in relation to both people and websites and how that confidence influenced their behaviours. Security orientation focused on respondents' general beliefs and feelings about security and its value. Finally, ego protection referred to the behaviours that respondents demonstrated in order to deflect perceived negative opinions about their security. In this section, each of these factors is examined in greater depth. In Chapter 5, the relationships between the internal factors as well as the effects of internal factors on awareness, perceived danger, and barriers and enablers is further elaborated on.

\subsubsection{Past Experiences with Breaches}

Prior experience focused on the perceptions of respondents about the past effects of perceived breaches. In many cases, respondents who believed they had experienced a cybersecurity breach in the past were influenced positively by this experience and tended to engage in safer behaviours than those who had not had such experiences. What was troubling was that respondents who did not believe that they had previously experienced a cybersecurity breach were often more lax about their security. For these respondents, the lack of a "catalyst event" often meant they were less concerned about their security than

Table 4-8. Respondent comments about whether they had experienced a cybersecurity breach in the past

\begin{tabular}{|c|c|}
\hline $\begin{array}{l}\text { Lowe } \\
\left(\mathrm{n}^{\mathrm{f}}=7\right) \\
\left(\mathrm{n}^{\mathrm{i}}=5\right)\end{array}$ & $\begin{array}{l}\text { "I've never had the virus yet, as far } \\
\text { as I know. Unless I have a virus and I } \\
\text { don't know it." (Par-3D) }\end{array}$ \\
\hline 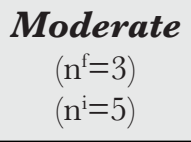 & $\begin{array}{l}\text { "No I don't think so. [Pause] Oh, } \\
\text { [pause] maybe. I think it might have } \\
\text { happened a long time ago" (Int-14) }\end{array}$ \\
\hline $\begin{array}{c}\text { High } \\
\left(\mathrm{n}^{\mathrm{f}}=4\right) \\
\left(\mathrm{n}^{\mathrm{i}}=10\right)\end{array}$ & $\begin{array}{l}\text { "[I have dealt with malware] } \\
\text { Numerous times in the past. Old } \\
\text { versions of Windows. And [I've] } \\
\text { cleaned up many other people's } \\
\text { computer or virus problems so I } \\
\text { definitely have seen a lot of different } \\
\text { viruses a large amount of times." } \\
(\text { Par-2A) }\end{array}$ \\
\hline
\end{tabular}
respondents who had experienced catalyst events. Furthermore, in some cases, respondents who had not experienced catalyst events were more likely to take risks because they had not seen any evidence that they would be faced with consequences for what could be considered unsecure behaviours.

Throughout the focus groups and interviews, respondents commented on how their past experiences with breaches had influenced their current behaviours. However, their experiences with previous breaches varied (see Table 
4-8). Approximately half of participants and one-quarter of interviewees believed that they had not experienced a cybersecurity breach. Half of the interviewees and approximately one-third of participants were confident that they had experienced a cybersecurity breach. The remainder of the respondents were unsure if they had experienced a breach of if the symptoms they associated with a breach were the result of malware.

Some respondents were unsure about whether or not they had experienced a breach in the past. Even those who were confident that they had not had such an experience still often expressed some doubt about whether or not they had experienced a breach without knowing it (see comment under "Low" in Table 4-8). These comments implied that respondents expected that clear symptoms would be present for most security breaches. More often, people who were unsure about their past experiences with breaches tended believe that they had experienced a breach (see comment under "Moderate" in Table 4-8, p. 102). However, despite the indication that the had experienced past breaches, similar levels of uncertainty were present with users who did not believe they had experienced a past breach. This uncertainty may be related to respondents' knowledge, which is discussed in the next section.

One of the issues associated with the uncertainty about past experience is that it was often associated with lower levels of concern about the danger of threats. One of the participants described how she was less concerned because there had not been a catalyst event to trigger a change in her behaviours:

We never locked our doors until somebody broke into this house. Now we lock our doors. We're never going to do anything about protecting ourselves on the Internet until we get nailed. (Par-2C)

Comments such as this indicate a strong need for a tangible sign that something had gone wrong before individuals were willing to change their behaviours. If nothing had gone wrong, respondents were less willing to take steps to protect themselves. This was further supported by a comment from the single respondent who did not use any safeguards on his home computer:

I wouldn't, I probably wouldn't go to the extent of purchasing something unless I was, or something happened. So it would be reactive rather than proactive. (Par-3A)

These comments and other similar comments implied a belief that there was no need to take protective steps until something happened, such as a catalyst event. Without such a catalyst event, respondents often felt the risk of a cybersecurity breach was lower, the potential consequences were relatively minor, and that there was little benefit of taking 
proactive security measures.

Respondents commonly noted how they considered the need for a catalyst event to be normal. Several participants discussed what it would take for them to take additional protective steps beyond what they were already doing:

Until you actually discuss it and you go through it. (Par-3D)

Until something happens. (Par-3B)

Until you go through a traumatic situation. (Par-3C)

I think that's the norm. (Par-3D)

Similar comments were echoed by the interviewees who also indicated that it would take some sort of major event before they would take additional protective steps. This underscored the need for the symptoms and consequences of a cybersecurity breach to be judged to be "severe enough" by the respondent before they would act. One of the interviewees elaborated on how severe the consequences would need to be before he would change his behaviours and become more proactive about his security:

The time taken at fixing it would have to be more than the time taken to put remedial steps in place. So if it would take me an hour a week to clean up, but it was only going to take me a half hour to do those extra steps then I would do the extra steps. (Int-17)

However, the prevailing opinion was that cybersecurity breaches were rare enough that apart from the steps requiring minimal time investments, taking protective steps to protect users' cybersecurity was not useful. This need for a catalyst event that has sufficiently severe consequences then helps to explain why respondents who had experienced what they considered to be minor breaches only took basic protective measures, such as installing antivirus software. Without the perception that the consequences were severe, respondents considered the breach to be more of an annoyance than anything else. Therefore, without sufficiently detrimental consequences, respondents were unwilling to put in the time, money, or effort to prevent the breach.

Accidents as a cause of security breaches were a topic that emerged during the focus groups. During two separate focus groups, participants stated that when they were browsing online, they became more concerned about their security when a website had remembered their password or they found that they were still logged into a website. One of the participants indicated:

I think it's scary when you bring up a site and it says "hello [Name]" (Par-3B) 
She further indicated that she was uncertain whether she had stayed logged on to the site, but because the site had recognised her, she worried that someone else had gained access to her account because of something she had done accidentally. During another focus group, two participants discussed how something that had been done "accidentally" caused one of the people to worry about her security:

When I logged on last week, my password was there. Once I punched in my ID, it automatically put in my password, and I was going 'what?' (Par-1A)

So you must've ticked 'yes' one day when you put in your [interrupted by participant 1A] (Par-1B)

Maybe, but I didn't know. Or maybe I accidentally clicked on it. (Par-1A)

Participants who experienced these types of situations were often quick to attribute the incident to an accident rather than them having actively done something that allowed the website or browser to remember their information. Respondents typically referred to "accidentally clicking" on things such as links, boxes to agree to things, or even popup ads. These sort of responses suggested that respondents had an underlying suspicion that what they had done was less than secure. However, they often distanced themselves from being associated with consciously unsafe behaviours which could result in negative consequences.

Due to the frequency with which participants discussed accidents, interviewees were specifically asked if they had ever accidentally done something that had put their cybersecurity at risk. Approximately one-third of interviewees indicated that they had done something accidentally. The remainder of the interviewees either said that they had not done anything that would specifically put their cybersecurity at risk or that these actions were consciously unsafe. The most commonly mentioned accidentally unsafe activity was "clicking on something" that the person "did not mean to," such as agreeing to terms and conditions, allowing a browser or website to remember login credentials, or clicking on ads.

Interviewees who blamed cybersecurity breaches on accidents more frequently seemed to have a diminished sense or personal responsibility when discussing who they felt was responsible for keeping them safe online. In contrast, interviewees who felt a greater responsibility for protecting their own cybersecurity tended to admit when their actions had caused a breach. One interviewee who felt strongly that protecting her internet security was her responsibility described an experience she had when she was a university student, 
when she felt that a breach was caused by her actions rather than an accident:

One of the things we would always do is never log on to a computer that someone has already logged on to. We would reset it and... you would go see each script and what other script it's executing. I didn't do that. So the computer that I logged on to had already, a student had written a program. And he captured my password. So if I had just followed the basic security procedures, shut down the computer, come through the batch file, I would've seen that unusual script. So it was certainly my fault. (Int-2)

Comments such as this one illustrate how blaming accidents for past experiences can also be linked to knowledge.

\subsubsection{Knowledge of Threats and Safeguards}

Knowledge was associated with respondents' understanding of threats and safeguards, including: how threats were propagated, the potential consequences, what safeguards could do, and how safeguards could be used. Respondents' knowledge of threats was influenced by their awareness of threats and safeguards since before understanding these, they must first be aware of them. Although an individual may have been aware of a threat, this did not imply further knowledge of the threat. Similarly, just because an individual was aware of a safeguard, it did not necessarily mean they knew how to use it to effectively prevent cybersecurity breaches. Because of the differences between awareness and knowledge, these topics need to be considered separately.

Knowledge was examined in relation to both threats and safeguards. Knowledge was evaluated based not only on how knowledgeable respondents considered themselves to be, but also on their descriptions of threats and safeguards and other statements indicative of their knowledge and understanding of the topic. This allowed their perceived knowledge to be compared with their actual / demonstrated knowledge, and to help determine if their perceptions of knowledge were accurate. In this section the emphasis is on perceived knowledge in order to examine how knowledgeable the respondents considered themselves to be. The confusion between actual knowledge and perceived knowledge is discussed in Chapters 5 and 6.

Respondents' knowledge of threats varied. However, most respondents considered themselves to have low to moderate knowledge of threats. Although respondents were often aware of the most common cybersecurity threats, they were not necessarily sure what the consequences were, how the threats were propagated, or more generally how 
they worked. When asked to describe the threats they were aware of in greater detail, respondents with lower levels of knowledge tended to respond with "I don't know," or "I'm not sure." (see comment under "Low" in Table 4-9). Respondents with higher levels of knowledge tended to provide more indepth answers about what threats were, and discussed why they needed to be knowledgeable about them (see comments under "Moderate" and "High" in Table 4-9). However, sometimes those who considered themselves to have high levels of knowledge provided responses similar to those made by respondents with lower levels of knowledge. These types of comments indicated a differences between perceived and actual knowledge.

Knowledge of safeguards was also an important part of respondents' security intentions because they needed to have enough of an understanding of the safeguards to know how to use them and why. Similarly to threat awareness, safeguard awareness varied with most respondents having moderate levels of safeguard knowledge. Such respondents typically understood the need for using safeguards, but did not necessarily know the specific steps they needed to take (see comment under "Moderate" in Table 4-10, p. 108). Most respondents were knowledgeable enough to have installed antivirus software. Only one participant indicated that he did not use any safeguards. Similarly to knowledge of threats, respondents with low levels of safeguard awareness provided very simple descriptions of the protective measures they could take. They also often remarked about their naiveté (see comment under "Low" in Table 4-10, p. 108). More knowledgeable respondents went into great detail about the steps they could take and extolled their security prowess (see comment under "High" in Table 4-10, p. 108). The comments made at both the high and low levels of knowledge suggested that there were other factors underlying the negativity about respondents' knowledge or their boasting, such as their levels of confidence in their skills, which could contribute to feelings of laziness or cause respondents to be more boastful. These issues are discussed in section 4.5.7. 
Table 4-10. Examples of respondent comments on their knowledge of cybersecurity safeguards

\begin{tabular}{|c|c|}
\hline \multicolumn{2}{|r|}{ Knozeledge (Safeguards) } \\
\hline $\begin{array}{l}\text { Lowe } \\
\left(\mathrm{n}^{\mathrm{f}}=7\right) \\
\left(\mathrm{n}^{\mathrm{i}}=8\right)\end{array}$ & $\begin{array}{l}\text { "I'd say I'm pretty naïve. I know very little about what could be there to protect } \\
\text { us." (Par-3D) }\end{array}$ \\
\hline $\begin{array}{c}\text { Moderate } \\
\left(\mathrm{n}^{\mathrm{f}}=4\right) \\
\left(\mathrm{n}^{\mathrm{i}}=6\right)\end{array}$ & $\begin{array}{l}\text { "I wouldn't know how to stop one myself if I didn't have some software } \\
\text { delivered to me... (Int-5) }\end{array}$ \\
\hline $\begin{array}{c}\text { High } \\
\left(\mathrm{n}^{\mathrm{f}}=3\right) \\
\left(\mathrm{n}^{\mathrm{i}}=6\right)\end{array}$ & $\begin{array}{l}\text { "I know a lot of things I could do [to protect myself]. I know how to change } \\
\text { the IP address and do all that if I really wanted to, and so that I know what my } \\
\text { keystrokes were. And I probably should because I know the technology is there. } \\
\text { As a matter of fact, I could do it right now with about } \$ 5 \mathrm{k} \text {, no } \$ 500 \text {, maybe } \\
\$ 600 \text {. I could check the keystrokes of just about anybody by setting up the right } \\
\text { kind of technology by finding out if they use a wireless [network], which is the } \\
\text { easiest one. And again, with the hard wire, it's not that much more technology } \\
\text { and I could find out whatever keystrokes they were making. And I know } \\
\text { somebody could do that to me. So I purposely think what I'm doing and don't } \\
\text { want anybody to know what I'm doing." (Par- } 2 E)\end{array}$ \\
\hline
\end{tabular}

Knowing where respondents obtained their information about both threats and safeguards from provided further insights into how they gained knowledge. Few respondents actively sought out security information without some sort of prompt. Prompts most often included the computer acting in unexpected ways or security messages from trusted parties. When respondents received those prompts, the responsibility for obtaining the relevant information was placed on the respondent. In most cases, respondents went to the Internet to get more information. However, some respondents realised the potential for that behaviour to be risky because of the potential for malicious users to essentially hijack what appeared to be legitimate information:

If I wanted to know how to make ourcomputer safe, I would probably Google it first. And then probably most likely it would be an Apple page [that would come up] because we have a Mac. (Par-1C)

But then, because weird things happen, you Google something then you click on the dodgy site. I mean, what's the level of trust we have in Google? (Par-1D)

I think that would be the classic sort of spyware malware site. How to improve security on your computer. ${ }^{2}$ (Par-1B) 
Although this group of individuals recognised the potential risk of blindly trusting that search results about malware would not be malicious, they were the only respondents who recognised this risk. However, the Internet was by far the most frequently mentioned source of information about potential security threats.

Although the Internet was the most extensively used source of information amongst respondents, the other sources they got information from and the order in which respondents sought this information varied by gender (see Table 4-11). Sources of information were one of the two areas that differed by gender. Reliance, the other area that differed slightly by gender is discussed later. Both men and women looked to people they considered to be more knowledgeable than themselves when seeking out information about security. Overall, it appeared that men put a greater emphasis on age than actual knowledge, assuming that younger people inherently had the necessary knowledge to protect themselves online, although they did seek out people with formalised qualifications for offering help, such as help desk support or IT experts. There tended to be a general assumption that people who were younger were more knowledgeable because they were more familiar with technology and the internet. Male respondents were more likely to seek out information from their children or other younger family members than female respondents. Approximately twothirds of male respondents indicated that they would seek out information from a child or younger relative while approximately one-fifth of female respondents indicated the same.

The main difference between male and female respondents was the likelihood of seeking information from their spouses or partners. Only one male interviewee indicated that he would ask his partner for information about computer security. He noted that it was because of "the work that my partner does" that made her a good source of security information. In contrast, female respondents were more likely to seek information from their spouses, especially if they considered them to be particularly knowledgeable due to their work. Female respondents tended to have a greater number of sources that they trusted than male respondents. While this may indicate that the male

Table 4-11. Most frequently cited sources of information (in descending order), by gender

\begin{tabular}{|c|l|l|}
\hline Rank & \multicolumn{1}{|c|}{ Men } & \multicolumn{1}{c|}{ Women } \\
\hline $\mathbf{1}$ & Internet & Internet \\
\hline $\mathbf{2}$ & $\begin{array}{l}\text { Children / } \\
\text { younger family } \\
\text { members }\end{array}$ & $\begin{array}{l}\text { Friends / trusted } \\
\text { acquaintances }\end{array}$ \\
\hline $\mathbf{3}$ & $\begin{array}{l}\text { Computer } \\
\text { experts (e.g. store } \\
\text { staff, work help } \\
\text { desk, security } \\
\text { companies, etc.) }\end{array}$ & Spouses \\
\hline $\mathbf{4}$ & -- & $\begin{array}{l}\text { Children / } \\
\text { younger family } \\
\text { members }\end{array}$ \\
\hline $\mathbf{5}$ & -- & $\begin{array}{l}\text { Computer } \\
\text { experts (e.g. store } \\
\text { staff, work help } \\
\text { desk, security } \\
\text { companies, etc. })\end{array}$ \\
\hline
\end{tabular}


respondents were more likely to take responsibility for ensuring their knowledge was up to date or that they were more discerning about their sources, it may also indicate a greater confidence in their knowledge. However, there is also a risk that if they do not have the information they need and do not have a source to get the information from, they may assume that whatever knowledge they have is sufficient to keep them safe.

Despite the fact that respondents sought information from those they considered to be more knowledgeable than themselves, there was a limit to how much more knowledgeable they wanted the person to be. During one of the focus groups there was a discussion about why several respondents would not seek out information from the IT staff at work:

Would you ask somebody, an IT person at work? I wouldn't. They're going to be like using the IT jargon and I'm going to be like "what are you talking about?" (Par-1D)

Par-1E nods in agreement

English, please! English! (Par-1C)

In part, their reluctance to approach people they considered to be highly knowledgeable seemed to be related to respondents' confidence in their own skills and their perceived knowledge. They did not feel that it would be effective to seek information from someone when they would not understand the information given to them. Their reluctance also appeared to be related to a desire to avoid feeling "stupid" as some respondents described when they talked about why they did not seek out more information:

I hate to admit it, but I am sort of ignorant on [how to protect my computer]. And I shouldn't be. But you know, the more you look into it, the more you learn, [and] the more you feel stupid that you haven't [done more to protect yourself]. (Par-3A)

This desire to avoid feeling "stupid" contributed to whether or not respondents were comfortable asking someone else for information. For example, in order to avoid being seen as "stupid" by those they worked with, respondents would avoid asking their advice on technical issues even if the person could have provided the most appropriate information.

Luck was often used to explain why respondents felt more or less susceptible to threats. However, the use of luck as an explanation was often tied to the amount of knowledge that respondents considered themselves to have. Luck was more closely linked to perceived knowledge rather than actual knowledge. One of the interviewees who considered herself to be only moderately knowledgeable but who demonstrated much higher levels of knowledge indicated that she felt that luck had a prominent role in keeping her safe from threats. In contrast, respondents who had greater levels of perceived knowledge were less likely to 
consider luck to be associated with susceptibility. For example, one of the interviewees with higher levels of knowledge and confidence in his ability to protect himself commented:

I think I'm pretty safe. And I think I know more than average about what's available... in that regard, I think that my knowledge has been more than adequate. Also my activities and how I am translating that awareness into my actions has been adequate [rather than luck]. (Int-4)

Positive comments about the effect of luck often served to explain why, in the absence of knowledge, respondents had not suffered from cybersecurity breaches. However, it may also have been used to cover up a sense of uncertainty about whether the steps that respondents were taking were the "right" steps.

Luck also appeared to have influenced users' likelihood of engaging in additional security behaviours. Comments such as "I'll just continue to press my luck" further emphasised the sense of fatalism associated with cybersecurity. These and similar comments illustrated how when respondents believed that their past behaviours combined with luck had so far protected them from threats, they were often unwilling to consider changing their behaviours in the future. This was true even if their past and current behaviours indicated that they were increasing the risk they would encounter a cybersecurity threat.

Knowledge of threats provided the basis for understanding when symptoms were actually associated with cybersecurity breaches. It also provided the basis for respondents to understand what the potential consequences of threats were, as it often encouraged them to seek out information on the threats. Without knowledge of both threats and safeguards, respondents were less likely to consider threats a danger and more likely to assume that whatever protective steps they had taken so far were sufficient. Although knowledge provided the necessary understanding of threats, in order to use that knowledge, respondents needed to have enough confidence in their ability to do so. Therefore, along with knowledge, moderate to high levels of confidence in respondents' own skills (perceived self-efficacy) were required before respondents were likely to actually engage in protective measures.

\subsubsection{Perceived Self-Efficacy and Confidence}

Overall, respondents tended to have moderate (6/14 participants, 6/20 interviewees) to high (4/14 participants, 9/20 interviewees) levels of perceived self-efficacy. Based on the literature, respondents were originally expected to have similar levels of knowledge and perceived self-efficacy. Respondents' perceptions of self-efficacy were often similar 


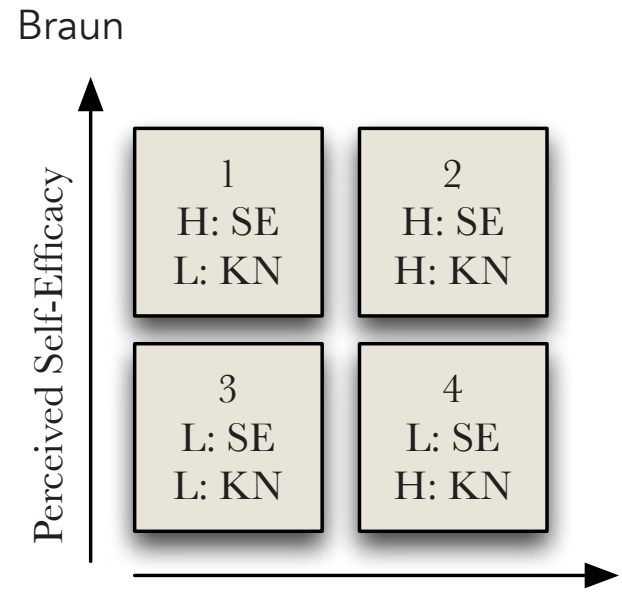

Demonstrated / Actual Knowledge

Figure 4-2. Perceived selfefficacy and demonstrated / actual knowledge

to their perceived levels of knowledge. Half of the interviewees and approximately two-thirds of the participants had similar levels of demonstrated / actual knowledge and perceived self-efficacy (see quadrants 2 and 3, Figure 4-2, p. 112). The remainder had either high demonstrated / actual knowledge with low perceived self-efficacy (quadrant 4, Figure 4-2) or low demonstrated / actual knowledge with high perceived self-efficacy (quadrant 1, Figure $4-2)$.

In many cases, it seemed that respondents' willingness to seek out information to increase their knowledge was driven by their confidence in their skills. The more the respondents sought out information, the more knowledgeable they typically were. An interviewee with moderate to high levels of both knowledge and perceived self-efficacy stated:

I'd say [I'm] pretty knowledgeable... I understand what the threats are. I understand enough about how they work to try to protect myself from them... I'd know where to go to try and find information [about how to get rid of a virus] and I might be able to do it in the end after a bit of effort. (Int-7)

In this case, the interviewee was confident enough not only in his ability to seek out the information but also to understand it. Because of that confidence he was more willing to try to take steps to remedy a security breach than other users who had lower levels of perceived self-efficacy. Respondents with higher levels of perceived self-efficacy often felt that they could not only identify but also fix possible problems, for example:

I can probably notice when something's more fishy and how I can clean it if it does pop up... I feel very confident that if something did occur, I could fix it. (Par-2A)

Respondents with lower levels of perceived self-efficacy were more likely to talk about not knowing "where to start" when it came to security issues. Respondents with greater levels of perceived self-efficacy understood the need for security, even if they did not have the knowledge or skill to carry it out themselves. Because of this, respondents with all but the lowest levels of perceived self-efficacy tended to understand the value of using security 
software, even if they were unsure of the effectiveness of it:

When I purchased my laptop, it said "do you want antivirus software?" I said yes because I know enough to know that it's important... But it doesn't mean I know how to personally do things about it. So naturally, I would just [pause] when I bought the laptop, I just said yes to the software to protect me. How much it actually protects me, I wouldn't have a clue. (Int-5)

It was common for respondents with low to moderate levels of perceived self-efficacy combined with low to moderate levels of knowledge to seek out someone or something to supplement their own skills, whether it was another person or protective software.

In some cases, respondents' perceived self-efficacy did not match up with their demonstrated knowledge, instead it matched their perceived knowledge. For example, 15 percent of interviewees and approximately 20 percent of participants considered themselves to have low to moderate levels of perceived self-efficacy and high levels of knowledge (quadrant 4, Figure 4-2, p. 112). Despite these beliefs, the knowledge demonstrated by these respondents tended to be much greater than most of the other respondents (quadrant 4, Figure 4-2, p. 112). These respondents tended to downplay their knowledge more than other respondents and typically indicated that they considered themselves to be less knowledgeable than the "normal user." One participant who considered himself to be poorly informed about threats, yet demonstrated a clear understanding and knowledge of the issues, stated:

I think I'm aware that it could happen any day. Like, despite what I do... if someone targets me and someone wants to do whatever, I think it could happen. (Int-2)

These respondents also tended to be more fatalistic about the possibility of having to deal with a security breach because they were not confident that their knowledge was sufficient to protect them. Similar statements were particularly common amongst respondents with low to moderate levels of perceived self-efficacy regardless of the level of knowledge they demonstrated.

Other respondents had higher levels of perceived self-efficacy but lower levels of knowledge (quadrant 1, Figure 4-2, p. 112). These respondents tended to overplay their skills more than other types of respondents. Approximately 30 percent of interviewees and 20 percent of respondents had moderate to high levels of perceived self-efficacy and perceived knowledge, but demonstrated lower levels of actual knowledge. These respondents often stated that they believe they could protect themselves from online threats because of their higher than average levels of skill. For example, one participant explained 
why he felt so confident in his ability to protect himself from threats:

You get so much information at work, and you take that home and you say, "well, I know what's going on because of work." (Par-2D)

In the above example, the individual assumed that the information obtained through work was sufficient to protect a home computer. However, he, and others who made similar comments, ignored the potential differences between home and work systems. The security assumptions about home computers made by these respondents were not always accurate. Despite the high levels of perceived knowledge and initial confidence in his skills, the above participant demonstrated a basic level of understanding of security threats and safeguard use. He also commented later that he would not know where to start in terms of how he could protect himself. Therefore, while perceived self-efficacy appears to be an important factor in influencing respondents to try to gain more information and in some cases to try taking new steps to protect themselves, high levels of perceived self-efficacy on their own are not necessarily indicative of safer behaviours.

\subsubsection{Reliance on Others}

Overall, respondents indicated a strong opinion that people were responsible for their own cybersecurity with every respondent indicating that they believed cybersecurity was a matter of personal responsibility. Initially this seemed to indicate that reliance on others would have little role in influencing respondents' cybersecurity intentions. However, throughout the focus groups and interviews, it appeared that these statements were made without any real conviction or sense of actual responsibility, for example:

It's up to me to ensure that I'm, that I have good internet habits I guess. If I rely on other people too much then who knows what can happen? I don't know. (Int-3)

Although the respondent indicated that he believed that security was his responsibility, the statement indicated that he was not really as certain about his responsibility as he professed. In many cases, despite respondents stating that they were responsible for their own cybersecurity, the reality was that throughout the discussions, respondents often made statements that they expected someone else to be responsible for at least some amount of their cybersecurity with approximately $70 \%$ of both participants and interviewees relying on someone else to protect their security to at least a moderate degree.

Respondents often expected that credit card companies and banks would protect 
them from the financial consequences of threats. However, when discussing who they expected to either prevent the threats or to remedy a breach if one occurred, they also relied on:

- family members,

- protective software (e.g. antivirus software),

- vendors of protective software, and

- website owners.

Reliance extended to increasing respondents' awareness of threats, taking preemptive steps to avoid threats, and in some cases, remedying breaches.

Respondents with lower levels of knowledge often demonstrated higher levels of reliance on others to provide them with what they considered to be necessary security information about threats. For example, approximately three-quarters of the female respondents indicated that they relied on their husband or another family member to protect their computer. When one of the interviewees talked about how she relied on her husband, she indicated that it was his knowledge and skill that made her do so. When discussing the steps she could take to protect her computer she commented:

I don't know. No! I really don't! I just trust my husband. He'll tell me if we need to take extra steps based on what he does at work... so I just trust him. (Int-13)

However, if the people being relied on were either unaware of the trust placed in them or did not take as extensive security steps as were expected of them, there was the potential for security problems. In a later interview with the husband of the above interviewee, he indicated that he relied on computer security companies for protection:

They are the experts. And I'm relying on them to keep my machine clean and free of viruses. And if it does get infected, clean it. (Int-17)

Thus, although his wife was depending on him to keep the computer safe, he was depending on someone else to do the job for him. Furthermore, his reliance on the security companies went beyond simple protection, and extended to remedying any breaches that occurred. In such a situation, it became apparent that if one person is expecting another to take additional steps to protect someone, the person being relied on needed to be aware of that reliance. Without that awareness, there was little guarantee that the reliant person's security was being taken care of adequately, nor was it clear where the final responsibility would lie. In addition, there remains a danger in relying on security companies since the end responsibility still lies with the user, limiting the amount that respondents could really 
expect to depend on the security companies. Furthermore, the amount of reliance that people place on others implies a certain level of trust that they will be protected.

\subsubsection{Trust in Websites and People}

Two aspects of trust emerged during the focus groups and interviews: trust in websites and trust in individuals. Trust in websites related to the trust that respondents had that the site itself would not cause their computer harm and in some cases, that the information contained on the site was legitimate. Trust of individuals referred to respondents' trust that the individuals they were interacting with did not actively seek to cause them harm. The presence of either type of trust influenced how respondents interacted with the person or website in question.

Respondents indicated that they were more likely to trust websites they were familiar with. There was an expectation that since the sites had not been harmful in the past, they would continue to be safe in the future. There was also an expectation that certain websites would be safe because of the type of websites they were (i.e. security sites, sites for large companies, etc.). For example, an interviewee described why she considered the Microsoft website to be trustworthy:

I know that the information they put out there, it might promote their own products. But at least they're not going to say things that are untrue. And they're not going to download viruses themselves. Or a company of that type [would be trustworthy]. (Int-14)

In this statement, the interviewee showed that her trust in websites extended to both the security of the site in terms of not providing malware, but also her trust in the information provided on the site. Another participant noted how she automatically saved login information on websites that she trusted, such as her university's page. In both of these situations there is a level of complacency that seems to have developed out of the trust. However, this sort of complacency can be dangerous if there is an expectation that certain websites should be trustworthy because of who they are run by if users forgo certain cybersecurity behaviours based on that trust. ${ }^{3}$ By foregoing these secure behaviours, respondents may have been making themselves more vulnerable to malicious users.

More broadly, trust can also be applied to individuals that respondents dealt with either directly or indirectly. Respondents demonstrated varying levels of trust of individuals

3 Malicious users have been known to actively seek out sites with good reputations on which to host malicious software or to mimic for phishing and pharming purposes (Symantec Corporation, 2009). 
online. Most considered themselves to be at least somewhat trusting of people online. One of the participants who considered himself to be generally trusting online explained:

I'm probably naïve enough to believe that that's, I trust people. That they wouldn't steal my ID or you know, take my credit card numbers and so forth. (Par-3A)

This comment suggested that the respondent felt he should have "known better" than to trust people. It also implied a certain level of discomfort with being so trusting. Higher levels of trust have the potential to be dangerous if people forego security behaviours in order to demonstrate that they trust an individual.

Approximately half of the respondents (6/14 participants, 11/20 interviewees) considered themselves to be cautiously trusting of people online. This set of respondents tended to avoid opening attachments from unknown senders and they generally did not interact with people they did not know. Although these behaviours offered some degree of protection, the danger arose when they were interacting with someone whose computer had been compromised or was not really the expected party. As one interviewee noted:

It was really frustrating this one time. I checked my email in the morning and I saw one from a friend of mine. I hadn't heard from her in ages, so I opened it up and it totally wasn't her... It happened a few more times... Now I've had to block that address. (Int-7)

The interviewee further went on to describe how although she usually did not open emails from people she did not know, she opened this one because she thought she knew the sender and chose to apply a different set of security behaviours than she otherwise would have. However, she also indicated that although it made her more cautious, it did not change her generally trust of people online.

Approximately one-third of the respondents (5/14 participants, 6/20 interviewees) considered themselves to be highly distrusting. The comments of one of the interviewees summarised many of the comments about why people were distrustful of those they did not know:

I think it's because, [pause] because of the physical distance. Unless you know the person, you just don't know. It could be anyone. (Int-1)

Without any physical context or preexisting relationship with a person respondents considered it to be very difficult to judge the other person's trustworthiness in an environment based on anonymity. In this sort of case it was not surprising that trust was not immediate. However, the distrust of people online at times even extended beyond strangers to people 
who could have been considered trusted contacts, such as family and friends. One of the interviewees described his levels of trust for both people he knew and did not know:

Even if I know [the person], I don't completely trust them. But I'm not saying I don't trust them on a personal level. For example, a lot of people on MSN and also on my Facebook they send me a lot of emails. But you know their account has been hacked or their computer has been infected by viruses. And even though you know they're a good friend, sometimes when they send me a link on MSN messenger or on Facebook, if it looks kind of dodgy to me, I don't trust them. And obviously, this sort of, you know, trust, becomes more apparent when you're dealing with people you don't know. (Int-4)

Although this level of distrust may result in greater security over time because of minimal interactions, this distrust of others could get to the point where it prohibited respondents from communicating with people in a normal way.

Overall, trust appeared to influence how respondents interacted with people and websites online. Furthermore, it contributed to the perceptions of danger that respondents felt with higher levels of trust being associated with lower levels of perceived danger. However, it is also useful in understanding trust to look at the factors that can increase and decrease trust of people and websites. These are discussed in Chapter 5.

\subsubsection{Concern about Security}

Respondents' feelings about threats and safeguards influenced their intention to engage in cybersecurity behaviours differently. Feelings towards threats tended to influence respondents' evaluations of the danger of threats (e.g. severity, susceptibility, and safeguard efficacy), while feelings about safeguards influenced their evaluation of danger as well as their beliefs about the benefits of using the safeguards.

Respondents feelings toward cybersecurity threats were negative, but the degree of negativity varied. Approximately one-third of participants and one-quarter of interviewees indicated that they considered many of the possible cybersecurity threats and associated consequences to be merely "annoying" and that malicious users were trying to demonstrate their skills to someone rather than acting in a truly malicious way. These respondents considered the threats to typically be benign and were considered to have a lower security orientation. For example, an interviewee talked about how she considered the consequences 
of a virus to be annoying:

You have a little wee document, and you think it's fine and you keep using it. Every once in a while, it won't open or it won't save or it won't work. And it turns out there's something in there and it's a really minor virus. And because you're copying and pasting this to make new documents, you're propagating it. And it's quite minor but quite annoying. (Int5)

Despite understanding that she was doing something to spread the virus, her concerns were not about what other things could have been happening as a result, bur rather the immediate issues that she could see with her document. Comments such as this often indicated a level of hope from respondents that the threats they faced were not as severe as they could be.

Some respondents were not particularly concerned about online threats at all. These respondents indicated that offline threats, such as having a credit card number stolen at a store or restaurant were more worthy of concern. In particular, the second and third focus groups were most likely to be concerned about offline threats, possibly because these were the threats they were most aware of since most had only been using the internet for a portion of their lives. One of the older participants discussed her feelings on offline threats:

Whether it's at the gas pump... or at the checkout at the grocery store or something like that, or the mailbox that they're going to steal my credit card bill, [I'm more concerned about that than threats] online. (Par-2C)

Typically people who were more concerned about offline threats had not been using the internet for as much of their lives (proportionately) as people who were more concerned about online threats. This was indicative of how age and extent of computer use influenced concern about threats.

Almost half the respondents stated that they found protective software or security behaviours to be annoying. These feelings were not limited to a particular safeguard or to a single type of safeguard. However, it did appear to be limited to safeguards where respondents believed they had to take steps to actively protect themselves online or where the safeguard they chose to use could prevent them from doing something they wanted to do. Respondents identified several safeguards that they considered to impact their ability 
to use their computers negatively, including:

\section{- Popup blockers}

We get popups on the computer at home. But I know that on a lot of PGs it will have a popup blocker, and it will go "This popup was blocked." But sometimes that can be annoying in itself... there are times when you do want the popup. (Par-1C)

- Turning off the computer when not in use

If you leave your computer on all night, it's open. It's on all night if you're on broadband. So what we do every night, once we finish, we turn everything off. It's annoying when you get up in the morning when you want to go on the internet. (Par1-D)

\section{- Conducting backups}

First of all, [conducting backups] is a pain. Right now, I've got about $500 \mathrm{~GB}$ of files on my computer. And the best practice is that you should at least back up your files once a week. And you think about the amount of time your computer needs to sit idle and do the backup... and if I do use the [online service]... 500GB [pause] how much is internet bandwidth going to cost me? So I consider that as the inconveniences. (Int-7)

Many of the comments about the annoyances of safeguards emerged when people justified why they did not use the safeguards. Many of these comments were echoed when discussing the barriers to engaging in better cybersecurity behaviours.

Although most respondents (24/34) felt that online security was part of today's culture and needed to be so, many of these same respondents tended to express more negative statements about cybersecurity than positive statements. These included comments about being unconcerned about security, not needing to take threats seriously, feeling like it was unlikely they would be targeted, and feeling that taking effective security measures was too difficult. Overall, there was a perception that security was something people should already know about and take into consideration. For example, several respondents talked about how people knew better than to trust the "Nigerian prince" offering to send money. Many of the feelings about security were focused on making security seem like something that people did not need to be worried about either because of the severity of threats, the susceptibility to threats, the goals of malicious users, or because they were skilled enough to prevent the threats. However, these reasons had the potential to lead to respondents 
engaging in ego protective behaviours to justify their non-use of safeguards.

\subsubsection{Ego Protection}

Respondents engaged in ego protection, often to divert attention from what were usually low levels of knowledge. In some cases, respondents were very boastful about their skills. While at times this boastfulness was justified, it more often appeared to be used as a way to draw attention away from something that the respondent believed they were lacking. Typically, respondents appeared to be trying to distance themselves from a lack of knowledge by boasting about how much they knew about threats online. However, these statements were often more directed towards the number of threats or safeguards that the respondents were aware of, rather than ones that they understood and could be considered knowledgeable about.

In some cases, respondents mistook awareness of threats for understanding and knowledge of how to avoid or remedy breaches. In almost every example of this, the respondents who engaged in these boastful behaviours credited their work or the fact that they had been using the internet for a long time as being responsible for what they considered to be their increased knowledge. When asked about why respondents considered themselves to be knowledgeable and confident in their skills, they often related it to something specific. However, respondents who were boasting without knowledge to back up their confidence $(5 / 34)$ tended to avoid elaborating on why they considered themselves knowledgeable, for example:

- Possibly just long experience with computers... (Int-15)

- Probably because of work... (Par-2D)

In in the first response, the interviewee appeared to be making the assumption that by simply using the internet for a long time he had increased his ability to avoid threats without having actively sought out information on the topic. The second response underscores the assumption that awareness of threats is the same as knowledge and the ability to protect against these threats. However, due to differences between the home and work environment as well as the differences in the types of threats that are associated with each, the simple awareness of threats is not necessarily enough to be considered knowledgeable. In both of these cases as well as in others, respondents made statements that attempted to detract from their lack of knowledge by instead playing up their awareness and boasting about that.

At the other end of ego protective behaviours, laziness, a common excuse (11/14 participants, 14/20 interviewees) was the reason why respondents did not take more steps 
to protect themselves online. Laziness was discussed in each of the focus groups by multiple participants and was identified by $70 \%$ of interviewees as the main reason why they did not do more to protect themselves. Several interviewees' comments summarised many of the other comments on laziness:

- Laziness is probably the main thing... knowing that there are probably steps I should be taking but I can't be bothered taking them. (Int-15)

- Lazy! Can I just tell you, lazy...You take the easy way out. I tend to take the easy way out. (Int-16)

- I know they're the major reasons that we don't change the password or take extra steps because we are lazy... (Int-9)

In this way, by using laziness as an explanation, respondents were still able to imply that they were still knowledgeable about safeguards, but that they preferred not to take steps because they did not see the value in doing so. Although laziness could be seen as negative, the fact that it still emphasised "knowing better" meant that it could be used in a way similar to boastfulness to distract from a lack of knowledge. Because of this, boastfulness and laziness were not mutually exclusive behaviours.

Boastfulness was typically associated with respondents who had higher levels of perceived self-efficacy, but lower levels of knowledge. By boasting, respondents were able to make it appear that they had more knowledge than they actually did, letting them present themselves in a more positive way. Boastful behaviours were more often associated with awareness and knowledge of threats and less commonly with safeguard use. In contrast, laziness was most often associated with respondents with lower levels of knowledge and was in almost every case associated with reasons why respondents did not engage in the steps they felt they should to protect themselves online.

\subsubsection{Summary of Internal Factors}

Seven main internal factors influencing home users' cybersecurity practice were identified during this study. As will be discussed in Chapter 5, these factors do not exist in isolation and can be influenced by other internal factors. In particular, respondents' past experiences, knowledge, and confidence in their skills appeared to have the greatest influence on their awareness and perceptions of danger. The relationships between the internal factors and perceived awareness and perceived danger are discussed in Chapter 5. Respondents internal factors can also be influenced by their external environment, such 
as symptoms of breaches, security messages, and other people.

\subsection{Influential External Factors}

Respondents' awareness of threats and their perceptions of danger were influenced by factors in their external environment. In the conceptual model, cues to action, encouragement by others, and use by others were expected to be included as external factors. However, encouragement and use by others did not appear to be as important to respondents in improving their security behaviours as other factors. The main factors that influenced respondents' awareness and perceptions of danger were symptoms, messages, and the context in which people used their computers (Figure 4-3). Symptoms refer to events that prompt individuals to take steps to protect themselves. These were either perceived or actual symptoms of a security breach which triggered a reaction by an individual in regards to their security. Messages refer to security-oriented communications that were pushed to individuals, including news stories or targeted awareness campaigns. These messages were typically delivered in the workplace, by family members who wanted to ensure their families were safe online, and from the media. The computer use context refers to whether or not an individual shared their computer with another person and how that influenced their security behaviours.

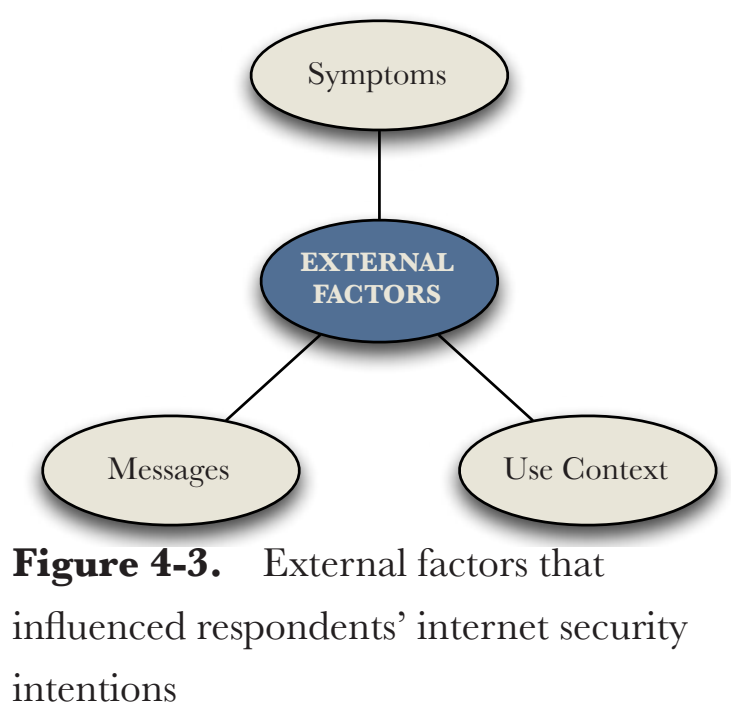

\subsubsection{Symptoms of Breaches}

In the cybersecurity context, the symptoms related to unexpected changes in the way that a computer functioned. Symptoms were differentiated from the internal factor of prior experience as symptoms refer to the initial signs of a breach, whereas prior experience relates to the overall consequences and respondents' feelings about those consequences that happen after a breach. Tangible symptoms of a possible security breach had the greatest influence of the external factors. Respondents often stated that because they had not experienced a security breach, they did not need to take additional steps to protect themselves. Respondents often judged whether a breach had taken placed based on whether 
or not they had experienced what they believed were the symptoms of a security breach.

A majority of both participants and interviewees stated that they were more compelled to use safeguards because in the past they had experienced the symptoms of what they considered to be cybersecurity breaches. However, the mere presence of minor symptoms was often not enough to actually cause respondents to take protective actions. For the symptoms to have the greatest effect, respondents needed to be able to clearly associate them with potential consequences and the symptoms themselves needed to be detrimental enough to cause respondents to act. The symptoms identified by respondents as being indicative of a security breach were increased numbers of popup ads and unexpected slow downs of respondents' computers. One interviewee described how the symptoms of a security breach triggered further action, but only after some time. However, it was not until these symptoms were associated with further consequences beyond the immediate problems with popups or computer speeds that this individual decided to take action after he could no longer open documents:

I opened a link and the computer just shut down. And when I restarted, it got really slow... it wasn't until later when I used [an antivirus program] to check the computer that I found out a lot of my files were infected. I needed to re-do everything. (Int-11)

This sort of response was common amongst respondents who ignored the possible symptoms of the breach until they could associate them with clear, tangible consequences. Even with the recognition of the consequences, respondents still required symptoms to be more than "minor symptoms" before they acted.

Although respondents indicated that they thought they may have experienced a breach due to the presence of particular symptoms, they often down-played the likelihood because they did not consider the symptoms to be detrimental enough to act upon. For example, a computer slowing down or the presence of pop-up advertising was not necessarily enough to influence respondents to be more concerned about their security. Instead, they were much more likely to take steps to protect themselves when they considered the symptoms to require the individual to expend a large amount of time, energy, or money to treat them. As another interviewee commented:

If I did lose my data or my laptop did melt down and I couldn't use it, or if I had to pay a whole lot of money and was incredibly inconvenienced by having to trot around government departments to prove who I really was... I think that would make me do much more [to protect myself]. It's just that I haven't [had to do that]... (Int-5) 
Without the more severe consequences of the person's life being disrupted, she was unlikely to take pre-emptive steps to protect herself online. These types of comments were frequently made by respondents and illustrate how the triggers themselves can influence perceptions of the severity of threats, leading to lower levels of perceived danger associated with threats. While symptoms had a strong effect on respondents' intention to engage in safer practices, security messages and the lack of these messages were also important.

\subsubsection{Security Messages}

Security messages were communications about the potential threats that individuals needed to be aware of and in some cases, steps that could be taken to either prevent or remedy the consequences of a breach. Security messages were considered to be messages that were pushed to the individual by someone else. The majority of respondents demonstrated a preference for having the information pushed to them rather than seeking it out themselves. Respondents most often received security messages from their place of work, followed by family members, and less frequently, the media.

One of the key differences between home and organisational users was that organisational users tended to receive more prompts about their security. As one participant explained:

At work, you're prompted to do something. At home, you're not. So unless the garbage stinks, you don't take it out. So something's got to prompt you to take the next step, or you don't [do it]. (Par-2C)

The lack of prompting and security messages in the home environment meant that respondents needed to take it upon themselves to find out information about security. Without prompts, users predominantly waited until they experienced symptoms of a breach, preferring to believe that they would not experience a breach in the future because it had not yet happened. However, comments such as the one above and other similar comments also illustrated the high level of dependence that individuals had on other people and organisations to make them aware of the possible risks online.

Building on this need for prompting, many of the respondents had at some point depended on their employers to provide them with the information they needed about security on their work computers. Respondents often assumed that if they were given this sort of information in their workplace, then the information would help to protect them at home. One of the interviewees described why she felt more knowledgeable about security 
as a result of her previous job:

I used to get messages in my old job that would come through that would say "this is a scam." You know, "this is spam, don't respond" or "this is a virus that is coming through" (Int-5)

As this comment and other similar comments illustrated, the security information that respondents received in their workplace was typically related to how to identify threats or who to contact in the event of a breach. However, in many cases the safeguard information provided and the reasons for taking those safeguards were minimal. One of the interviewees recounted how at work he was told to stop accessing any external websites in order to avoid a web-based threat that his employer was aware of, but he was not provided with information about why it was necessary. Messages such as this led to confusion about how this would help protect the computer. The main risk with regarding employers as the main source of information was that it led some respondents to expect that they would be notified of the "real" security threats by their workplace and that the information they received there would be enough to protect them at home. Because of this, they also tended to discount information that did not come from their employer.

Security messages obtained through the workplace were often not limited to just employees. In many cases, people who received security notifications at work sent these messages on to their family members. The people sending the messages felt that they were being helpful by keeping their family members informed about security even if they did not necessarily understand the actual security message itself. One of the interviewees described this practice:

I get emails at work from people about viruses and things. Then I'll forward them to [my husband] at home. The alert of the virus or whatever it is. (Int-19)

In a later interview with her husband, he did not mention these messages as being a source of information. This raises a question about whether or not people who received security messages in this way consider them to be relevant and valuable or if there are other reasons why they choose not to act on them. Throughout the data collection, the respondents who discussed the value of messages forwarded from work to another individual were the people who were forwarding the messages rather than recipients of those messages.

Alongside messages from work or in place of them, some respondents indicated a dependence on the media to inform them of the most dangerous threats. A comment by 
a participant summed up many similar comments:

The media is pretty good about telling people about the super viruses, and you know, all the time you need to protect yourself. (Par-1E)

A further comment by an interviewee underscored the importance of the media when she talked about how it provided her with information that she would not otherwise seek out on her own:

I wouldn't have known about identity theft if it hadn't been for the media. I would know nothing about it and I couldn't care less. (Int-13)

Although identity theft on its own was not a cybersecurity threat, the interviewee was aware of it as a consequence because of the news. However, further discussions showed an uncertainty about how the identity theft would occur or how it could be prevented.

Surprisingly, pop-up security warnings were not considered to provide useful messages. Respondents who mentioned these types of warnings typically only highlighted that they ignored them. Respondents who felt they did not understand security well often believed that they would not be able to understand the message, and therefore did not feel that they would benefit from paying attention to what it said. However, overall, few comments and even fewer positive comments about the effects of security messages were made.

Overall, while security messages were at times helpful to respondents, the respondents needed to feel that there was value in the messages before they would actually do something to protect their security. In most cases, respondents simply considered security messages as a way to increase their awareness about threats. However, most did not actually take any additional protective steps on their home computers as a result of these messages.

\subsubsection{Computer Sharing}

Computer use context had to do with whether the respondent shared a computer with someone else and whether they considered themselves to be the main person responsible for taking care of the computer. Discussions surrounding how a shared computer had the potential to influence respondents' security behaviours first emerged during the focus groups. Those comments were mostly made in passing. While these comments were included in the coding scheme, they did not appear to be of particular importance until the data were revisited after the completion of the interviews. During the interviews, 
4 of the first 5 interviewees also indicated that sharing a computer with other family members either changed or influenced their security behaviours. As a result, all subsequent interviewees were specifically asked about whether they shared their computer and whether that influenced the steps they took to protect themselves and the shared computer.

When respondents shared their computer with someone they considered to be more knowledgeable and skilled than they were, they often deferred to that individual and gave them the responsibility for protecting the computer. One interviewee's comments summarised many of the similar comments by people who shared a computer but did not consider themselves to be responsible for its security:

I have a husband who is a network engineer who looks after the things like making sure we've got antivirus software installed and that it's sort of automatically updating itself. (Int-13)

This interviewee was discussing why she was less aware of threats. However, these types of comments were common amongst those who depended on someone else to protect their security and they demonstrate one of the ways that respondents tended to justify distancing themselves from taking responsibility for their own security.

Respondents who considered themselves to have the primary responsibility for protecting the computer often responded with either active or passive security behaviours in response to the behaviours of the person they shared the computer with. There were also indications that people engaged in a combination of active and passive security behaviours. These behaviours are summarised in Table 4-12, p. 129. Active behaviours refer to respondents proactively taking steps to protect a shared computer from cybersecurity threats. Passive behaviours refer to respondents engaging in a more laissez-faire environment where each individual in the household was allowed to use the computer however they wished without any interference from the other users in regards to cybersecurity. These behaviours took place either before the computer was used by the respondent or the shared user, while it was being used by other people in the household, or when the respondent started to use the computer after it had been used by another member of the household.

Active security behaviours were performed either unilaterally without the input of others or in cooperation with other users. One of the interviewees described how he only gave family members limited accounts in order to limit what they could install on the computer, a very unilateral behaviour. In contrast, another interviewee described one of the more co-operative behaviours that she and her husband engaged in to protect their 
Table 4-12. Summary of computer sharing behaviours based on security response types (active, passive, or combination) and when those steps were taken

\begin{tabular}{|c|c|c|c|c|}
\hline & $\begin{array}{c}\text { Before Computer } \\
\text { Use (Proactive) }\end{array}$ & $\begin{array}{c}\text { During Computer } \\
\text { Use }\end{array}$ & $\begin{array}{c}\text { After Computer } \\
\text { Use (Reactive) }\end{array}$ \\
\hline \multirow[t]{2}{*}{ Active } & $\begin{array}{c}\text { Unilateral } \\
\left.\qquad \mathrm{n}_{\mathrm{f}}^{\mathrm{f}}=1\right) \\
\left(\mathrm{n}^{\mathrm{i}}=3\right)\end{array}$ & $\begin{array}{l}\text { Unilateral protective } \\
\text { behaviours before } \\
\text { using the computer } \\
\text { online or allowing } \\
\text { someone else to use } \\
\text { the computer online. }\end{array}$ & $\begin{array}{l}\text { Unilateral protective } \\
\text { behaviours while } \\
\text { using the computer } \\
\text { online. }\end{array}$ & $\begin{array}{l}\text { Unilateral protective } \\
\text { behaviours taken } \\
\text { after another person } \\
\text { has used the shared } \\
\text { computer online. }\end{array}$ \\
\hline & $\begin{array}{c}\text { Cooperative } \\
\qquad \begin{array}{c}\left(\mathrm{n}^{\mathrm{f}}=1\right) \\
\left(\mathrm{n}^{\mathrm{i}}=5\right)\end{array}\end{array}$ & $\begin{array}{l}\text { Agreed upon } \\
\text { protective steps } \\
\text { created in } \\
\text { cooperation with } \\
\text { others that should be } \\
\text { utilised before using } \\
\text { the computer online. }\end{array}$ & $\begin{array}{l}\text { Steps taken in } \\
\text { cooperation with } \\
\text { other users while } \\
\text { using the computer } \\
\text { online. }\end{array}$ & $\begin{array}{l}\text { Implement agreed } \\
\text { upon protective } \\
\text { steps to be taken in } \\
\text { cooperation with } \\
\text { other users that } \\
\text { should be taken after } \\
\text { the shared computer } \\
\text { has been used online. }\end{array}$ \\
\hline \multicolumn{2}{|c|}{$\begin{array}{c}\text { Passive } \\
\left(\mathrm{n}^{\mathrm{f}}=0\right) \\
\left(\mathrm{n}^{\mathrm{i}}=2\right)\end{array}$} & $\begin{array}{l}\text { Accepting of } \\
\text { other users' online } \\
\text { behaviours. No } \\
\text { proactive steps taken. }\end{array}$ & $\begin{array}{l}\text { Accepting of } \\
\text { other users' online } \\
\text { behaviours, no } \\
\text { additional safeguards } \\
\text { taken. }\end{array}$ & $\begin{array}{l}\text { Accepting of } \\
\text { other users' online } \\
\text { behaviours, no } \\
\text { additional protective } \\
\text { steps taken after the } \\
\text { shared computer has } \\
\text { been used. }\end{array}$ \\
\hline \multicolumn{2}{|c|}{$\begin{array}{c}\text { Active / Passive } \\
\text { Combination } \\
\left(\mathrm{n}^{\mathrm{f}}=12\right) \\
\left(\mathrm{n}^{\mathrm{i}}=10\right)\end{array}$} & $\begin{array}{l}\text { Accepting of other } \\
\text { users' behaviours, but } \\
\text { proactive steps taken } \\
\text { to protect against } \\
\text { something another } \\
\text { user might do while } \\
\text { using the computer. }\end{array}$ & $\begin{array}{l}\text { Accepting of } \\
\text { other users' online } \\
\text { behaviours. }\end{array}$ & $\begin{array}{l}\text { Accepting of other } \\
\text { users' behaviours, but } \\
\text { additional protective } \\
\text { steps taken to ensure } \\
\text { the computer's } \\
\text { security after it has } \\
\text { been used by another } \\
\text { person. }\end{array}$ \\
\hline
\end{tabular}

computer:

Let's say we receive emails from someone that we don't know.

My husband will ask me if I know the person. So we make sure that we don't open it. (Int-12)

Although this was an example of the fairly minor security behaviour of not opening emails when unsure of the source, it was one of many similar comments about the types of shared security behaviours that respondents' engaged in. Compared to other security behaviours, these tended to be based on behavioural safeguards rather than technological safeguards. The decision about whether to engage in unilateral or cooperative active security depended primarily on the descriptions of household dynamics and the way in which the parties 
interacted with others they shared the computer with.

Passive computer security meant that other people using the shared computer were able to do as they wished, regardless of any potential breaches that could have caused harm to other users of the computer. Passive security behaviours did not mean that the primary user of the computer was unconcerned about security, only that they did not take additional steps to prevent a potential breach that could have been caused by another person using the computer. These passive security behaviours were typically done for reasons related to preserving harmony in the relationship between users. For example, one interviewee discussed his unwillingness to take security steps because he did not want to show that he distrusted the other person's security behaviours:

I share my computer with my partner, and he is not very security aware. So, a lot of the time I'm really concerned that he uses my computer and that he has not really put security as a priority. And because of this, it might compromise my files. But on the other hand, because you have the partnership, you want to basically demonstrate your trust in the other person, when in fact you don't really trust them. So that's the dilemma there. (Int-4)

In this case, the interviewee opted to avoid using the computer for financial transactions, but continued to use it for everything else. Even though interviewees such as the one described above indicated that they were concerned about their security, it was placed as a lower priority in order to maintain household happiness. These behaviours were the least commonly seen with only 2 of the 14 interviewees who specifically discussed computer sharing. Respondents who were more passive tended to have laxer security behaviours on their shared computer than other types of users because they wanted to be accommodating to others.

The most frequently described computer security behaviours with a shared computer were a combination of active and passive behaviours. Almost all participants and half of interviewees adopted this approach. The steps taken in relation to combination behaviours were predominantly reactive with respondents taking security steps to remedy any potential issues after someone else had used the computer. One of the interviewees described what she regularly did after her husband had used their computer and why she chose to engage 
in a combination of active and passive behaviours:

It's really difficult to be a cop. You can't sort of tell him "don't go to this site," "don't go there," don't do this," don't do that." It's just really difficult. So I just let him do whatever he can do, and each time at night before I log in, I just go through the history and go through what's been there, what he has downloaded, what has happened. (Int-18)

In most cases, the user who was primarily responsible for the household's computer security could accept the other users' potentially risky behaviours. However, once the other person was no longer using the computer, the respondent would take reactive steps to ensure that nothing that the other person had done would have a detrimental impact on their data. Unlike the passive behaviours where the goal was to ensure a harmonious household, in this situation the security of the data was given a greater importance. With combination security, there tended to be a sense of resignation that regardless of what a person did to protect their own cybersecurity it could be undone by other users of the computer. However, unlike passive behaviours where this realisation was met by a lack of action on the respondent's part, in this situation the response tended to be that people believed that the next best thing to engaging in active security behaviours was to take unilateral steps to protect the computer when the respondent was using it, regardless of the potential household consequences.

\subsubsection{Summary of External Factors}

The most prevalent external factors, symptoms and security messages, focused on prompts that caused respondents to reconsider the steps they were taking. In many cases, the external factors were precursors to the internal factors that influenced respondents' behaviours. For example, a respondent typically experienced some sort of symptoms of a security breach before forming opinions about how the experience affected them. Similarly, respondents who did not experience symptoms tended to be affected in a very different way, focusing instead on the lack of symptoms meaning they were safe. Furthermore, computer use context had the potential to foster high levels of dependence on someone else to maintain the computer. Therefore, it is important to consider the effect of external factors on awareness, perceptions of danger, and internal factors. 


\subsection{Perceived Barriers \& Enablers}

Perceived barriers refers to the reasons why users do not take more steps to protect themselves online while perceived enablers are additional reasons why people did take protective steps. Perceived barriers and enablers are analysed together because in many cases respondents described a simplified cost-benefit analysis to determine if the reasons to protect themselves outweighed the costs of doing so.

\subsubsection{Perceived Barriers}

Respondents in both the focus groups and interviews were asked to identify the barriers that stopped them from engaging in better security behaviours online. As noted in Chapter 3, participants were asked to rank the top three most substantial barriers to engaging in cybersecurity behaviours from most important to least in order to determine

Table 4-13. Top ranked barriers to engaging in cybersecurity barriers (in descending order)

\begin{tabular}{|c|l|l|l|}
\hline Rank & $\begin{array}{c}\text { Group 1 } \\
\text { Part-Time } \\
\text { Graduate } \\
\text { Students }\end{array}$ & $\begin{array}{c}\text { Group 2 } \\
\text { Working } \\
\text { Professionals }\end{array}$ & $\begin{array}{c}\text { Group 3 } \\
\text { Retirees } \\
\text { \& Older } \\
\text { Participants }\end{array}$ \\
\hline $\mathbf{1}$ & Ignorance & Laziness & Ignorance \\
\hline $\mathbf{2}$ & Financial Cost & Time & Knowledge \\
\hline $\mathbf{3}$ & Time & $\begin{array}{l}\text { Lack of } \\
\text { Prompting }\end{array}$ & Financial Cost \\
\hline
\end{tabular}

The overall outcome of the focus groups was that the most frequently mentioned barriers based on the number of people who raised the barriers in discussion (in descending order) were:

1. laziness,

2. ignorance (e.g. the lack of knowledge about what respondents felt they should know),

3. lack of knowledge (e.g. the lack of knowledge and understanding of how to actually take protective steps),

4. time required to protect the computer,

5. cost of protective software, and

6. lack of prompting about security. 
The topics identified were similar in both the participant ranked lists and the list of most frequently discussed barriers. However, the ranking of the barriers differed. Comparatively, laziness was identified and discussed as a barrier much more frequently than any other barrier. Laziness was identified as a barrier by approximately three-quarters of respondents (11/14 participants and 14/20 interviewees). However, it only appeared in the top 3 list of one of the focus groups.

Comparing the most frequently mentioned barriers from participants to those of interviewees, the barriers identified were the same. However, they were mentioned with differing frequencies. In the interviews, the top 3 most extensively discussed barriers based on the number of interviewees who discussed them were:

1. laziness,

2. ignorance, and

3. time associated with better cybersecurity behaviours.

Interviewees were slightly more concerned about the time required to take the necessary protective steps than participants appeared to be. However, the overall frequency with which the barriers were discussed was nearly identical for both participants and interviewees.

What stood out from the top identified barriers was that many of the barriers were more closely related to internal factors than specific barriers. In particular, ignorance, knowledge, and laziness were all factors internal to the individual. While these factors may have inhibited respondents' security behaviours, they went beyond just influencing barriers. For example, although a lack of knowledge was frequently considered to be a barrier, in many cases this lack of knowledge contributed more to respondents' awareness and their overall perceptions of the danger of threats. As a result of lower perceived danger, respondents were less likely to engage in as comprehensive security behaviours as people who had higher perceptions of danger. Additionally, respondents with lower levels of perceived danger tended to be more affected by barriers. The remaining barriers of time, financial cost, and lack of prompting are external to the individual and are the focus of the remainder of this section.

The time cost associated with engaging in protective behaviours was the greatest barrier to respondents' engaging in such behaviours. This barrier ranked third and fourth for interviewees and respondents respectively. The time associated with engaging in cybersecurity behaviours can refer to the time it takes to gain the necessary knowledge and awareness as well as the time it takes to implement the safeguards. Respondents often described how learning about the threats and safeguards was too time consuming. As one 
of the participants noted:

There's probably a new virus created every 15 or 20 seconds, and I doubt it's any less than that. Who's got the time in the day to keep track of it all? You don't! (Par-2E)

These sorts of comments were often made by respondents who were comfortable with their levels of knowledge, even though they tended to be basic and at times verged on outdated. However, even when respondents knew what steps could be taken to protect themselves, time was still a barrier to engaging in protective behaviours. One of the interviewees who was most concerned about the time requirements described how he assessed whether or not to use a particular safeguard:

The time taken at fixing [the results of a breach] would have to be more than the time taken to put remedial steps in place. If it took me an hour a week to clear up, but it was only going to take me a half hour to do the extra steps, then I would do the extra steps. (Int-6)

As a result, he only used the safeguards that he considered to be the most efficient and effective, opting to use the ones that provided the clearest benefits.

Financial costs of purchasing protective software also created barriers to use. One participant commented on why she believed that people did not take steps to secure themselves:

People don't do security because they can't afford it. (Par-1E)

Respondents often agreed with the sentiment that practising security was expensive. For example, an interviewee elaborated on why she had not updated her husband's or son's computer with up to date antivirus software:

We have to pay every time we update the antivirus software. So because of that we [don't update it]... unless we have a free software for that... but I don't think so for less important things. (Int-1)

Another interviewee noted that his lack of antivirus software changed the way he used his computer:

The cost of installing security on my Mac is quite a bit more than installing my PC, so I only use my Mac to access the internet. (Int-11)

In this case, he opted to limit the ways that he used his Mac, effectively reducing its 
functionality rather than installing protective software. While respondents in both of these situations still used security software on at least one of their machines, they opted for selective use and minimal protections because of the cost of the protective software.

Although free alternatives exist, many respondents felt that they were not as effective as their paid counterparts:

I think we know what we could be doing, but we do it [practise security] the free way. The other ways cost money. (Par-2B)

Throughout the discussion, this participant talked about how his computer could be better protected by costly software. However, he did not consider the additional cost to provide a sufficiently higher level of protection to justify the additional spending. Despite the presence of free software, cost was still a barrier for respondents because they did not consider the effectiveness of free software to be as good as with paid software. Furthermore, some respondents felt that free protective software had additional drawbacks, such as slowing the computer down. This bias against free software often meant that respondents did not feel that this was an effective way to overcome the barrier of cost.

A final barrier identified by participants and interviewees was the lack of prompting that users receive about security threats. During the second focus group several participants engaged in a discussion about the prompting received at work to change passwords:

At work it's because they say your password's expired and you have to do it. At home, [my husband] takes care of it. So, I don't know. Do they tell you your password's expired someplace and you have to change it? (Par-2C)

I haven't run into that password's expired. (Par-2E)

Yeah, that's fair. Very few. I mean, at work, they force you to change passwords. (Par-2D)

Which I don't do. (Par-2A)

I don't know of anybody, any site that forces you to change your password. (Par-2D)

Similar comments arose in the third focus group as well as in one-quarter (4/20) of the interviews. Prompts from security software helped to remind respondents that there was protective software running on the computer. In many cases, respondents only received prompts from the software when something went wrong, such as a security incident or the 
security software expiring. For example:

[My security software] does tell me every now and then that I have problems and then I have to open it and find out how to fix it, and I'm trying to think. There's a website trying to access your homepage. Or trying to change your home page all the time, I get that all the time. (Par-3C)

However, in some cases, it was noted that people looked for the visual clue of a green checkmark to tell them that everything on their computer was fine. Prompts were one of the main areas where home and organisational users differed. Respondents indicated that when they were working or using a work computer they received prompts about security threats and the need to change passwords. This increased their awareness. However, outside of the organisation, respondents often did not receive similar prompts unless they had a person who regularly passed on relevant security information to them or they visited a website that required them to change their passwords regularly. Despite the presence of barriers, there were some factors that encouraged respondents to engage in more protective behaviours online.

\subsubsection{Perceived Enablers}

When discussing the barriers to and enablers of engaging in better cybersecurity behaviours, participants and interviewees focused predominantly on the barriers rather than the benefits. This may be because many of the benefits associated with cybersecurity are intangible. One interviewee commented:

In security, the intangible benefits for a home user are [pause] because, again, since they're intangible, you may not sort of be aware of them. (Int-4)

The intangible nature of cybersecurity benefits may mean that users do not have any indication or sign that the behaviours they are engaging in are correct.

Another interviewee noted why he engaged in what he considered to be safer behaviours online:

I want to basically stay safe and not have to deal with those kind of issues if my computer did get infected. (Int-3)

The issues the interviewee was referring to included the time associated with repairing the consequences of a cybersecurity breach or the cost of fixing the computer or restoring the data. Avoiding consequences such as buying products to fix problems, being without 
a computer, and having to take the time to fix problems were the main reasons why respondents utilised safeguards.

Neither participants nor interviewees appeared to have a strong sense of the possible benefits of engaging in cybersecurity behaviours. Instead there was a focus on the broader reasons of what consequences participants and interviewees wanted to prevent from occurring. Many respondents related their computer security to their physical security when talking about the benefits. For example:

You know, just like the steps you take to protect your house from being vandalised, you have a burglar alarm system, it just gives you reassurance that you're not going to experience a theft or robbery or whatever it might be. Just for piece of mind, it just gives you one less thing to worry about. (Par-3A)

The focus on broad benefits also came through at the end of the focus group sessions when participants identified the main reasons they wanted to engage in cybersecurity behaviours (see Table 4-14). Of the reasons given for protecting themselves, participants most were focused on the tangible aspects of what they could protect. For example, protecting against financial risk could have tangible consequences if the person noticed that there are unwanted charges on a credit card. Avoiding financial risk and the associated consequences were the most frequently seen reasons overall for participants to actively take steps to protect their cybersecurity.

The three focus groups varied in what reasons enabled them to engage in better behaviours online. The first group focused on tangible threats that the participants felt protective behaviours could protect against. The second group, in contrast was more influenced by the experience and knowledge that they had gained over their time online. The final group focused more on the emotional security that Table 4-14. Top ranked reasons to engage in utilising protective behaviours could provide. Interviewees indicated similar reasons for engaging in cybersecurity behaviours. The primary reasons amongst interviewees were to avoid financial consequences due to cybersecurity breaches and to maintain a feeling of security cybersecurity behaviours (in descending order)

\begin{tabular}{|c|l|l|l|}
\hline Rank & $\begin{array}{c}\text { Group 1 } \\
\text { Part-Time } \\
\text { Graduate } \\
\text { Students }\end{array}$ & $\begin{array}{c}\text { Group 2 } \\
\text { Working } \\
\text { Professionals }\end{array}$ & $\begin{array}{c}\text { Group 3 } \\
\text { Retirees } \\
\text { \& Older } \\
\text { Participants }\end{array}$ \\
\hline $\mathbf{1}$ & $\begin{array}{l}\text { Avoid financial } \\
\text { risk }\end{array}$ & $\begin{array}{l}\text { Prior } \\
\text { experiences }\end{array}$ & $\begin{array}{l}\text { General desire } \\
\text { for protection }\end{array}$ \\
\hline $\mathbf{2}$ & $\begin{array}{l}\text { Avoid } \\
\text { reputation risk }\end{array}$ & $\begin{array}{l}\text { Knowledge } \\
\text { of possible } \\
\text { consequences }\end{array}$ & $\begin{array}{l}\text { Avoid feeling } \\
\text { vulnerable }\end{array}$ \\
\hline $\mathbf{3}$ & Protect children & $\begin{array}{l}\text { Avoid financial } \\
\text { risk }\end{array}$ & $\begin{array}{l}\text { Avoid perceived } \\
\text { consequences }\end{array}$ \\
\hline
\end{tabular}
when online. 


\subsubsection{Summary of Perceived Barriers \& Enablers}

Overall, the barriers to and enablers of security behaviours tended to be assessed together in a basic cost-benefit analysis. In that analysis, the cost was the time and financial costs of engaging in safer behaviours while the benefits were avoiding the time, financial, and emotional costs associated with a breach. In many cases, respondents considered a lack of many of the internal factors as barriers to engaging in security behaviours. Commonly, a lack of knowledge was cited as being one of the main reasons why people did not do more to protect themselves. This illustrated how lower levels of many of the internal factors were tied to a lower levels of perceived danger and higher perceptions of barriers. In many cases, it appeared that respondents struggled to enumerate the actual benefits associated with protecting themselves. This may be because with security, the typical benefit is to protect the status quo. Thus, it appeared that for those respondents who did identify benefits, these benefits were not always benefits for themselves, but rather for their families and children. Therefore, when considering the effect of barriers to and enablers of users' cybersecurity intentions, it seems that the barriers had a greater effect.

\subsection{Cybersecurity Intentions \& Behaviours}

The most commonly identified security behaviours that respondents engaged in (in descending order) were:

1. using antivirus software;

2. not opening email attachments from unknown senders;

3. using a firewall; and

4. only visiting "reputable" websites.

The most common security behaviour was the use of antivirus software followed by not opening unknown email attachments. Two interviewees stated that they utilised all of the safeguards they were aware of. The remaining interviewees indicated that there were safeguards they knew they should use but either chose not use them or were unsure of what those steps were.

During the focus groups, participants frequently noted that there were things they could do to protect themselves online, but that they chose not to. Often comments were made about either knowing or not knowing what the "best practices" were and whether 
the participants thought they were living up to that standard. Given the extensiveness of comments about best practices during the focus groups, interviewees were specifically asked about whether there were additional steps they knew of that they could take to protect themselves, what those steps were, and what they considered the ideal security practices to be.

More than half of interviewees $(11 / 20)$ indicated that they were unsure of or unaware of any additional steps. In a comment which summarised many of the other comments, one interviewee stated:

I should be doing more, but I wouldn't even know what more I should be doing really. It's just sort of a general feeling of, you know, there's some golden standard and I'm sure I don't meet it. And I'm sure neither do the rest of the people in the family. (Int-5)

When interviewees were unable to identify additional steps they could take but they were not already doing, they often recounted the steps they took and indicated these were the steps they believed made up the best practices. The best practices that were most commonly identified in descending order were:

- having antivirus software installed (11/11),

- not opening email attachments from unknown sources $(10 / 11)$,

- not visiting sites that they believed were more likely to host malware (e.g. not visiting pornographic websites) (6/11), and

- having a firewall installed on the computer (4/11).

Of the interviewees who indicated that they were following all of the best practices that they were aware of, most also indicated that they felt they were less aware of how they could protect their computers and their data than the normal user.

The remainder of the interviewees (9/11) indicated that they were aware of things that they could do to protect themselves but that they did not do because of one or more of the barriers identified in the previous section or because they did not consider the danger to be high enough. Like the interviewees who were unsure of the steps they could take, all of the remaining interviewees indicated that utilising at least antivirus software was part of what they considered to be best practices. Utilising a firewall was mentioned more frequently by this group of interviewees with 8 of the 9 interviewees indicating that they 
use a firewall in addition to antivirus software. Other suggested best practices included:

- not sharing the computer with other users,

- conducting weekly backups of all their data,

- turning off the computer when not actively using it,

- turning off access to services that could be exploited by malicious users,

- utilising advanced methods to prevent malicious access, such as the use of honeypots ${ }^{4}$,

- only visiting trusted sites,

- only downloading from trusted sites, and

- not providing personal information online.

Overall, there was no agreement about what the best steps to take were. Most of the best practices listed above were identified by only one or two of the interviewees, though several focus group participants also indicated that they felt that turning the computer off when not using it would provide additional protection. These mixed responses are indicative of a general lack of clarity about what steps should be taken to protect home computers and what steps are the most effective.

Respondents' intentions to engage in security behaviours were influenced by their perceptions of danger, the perceived barriers to engaging in secure practices, and the factors that enabled them to overcome these barriers. If the perceived danger of the threat was not high enough, respondents were less likely to engage in safer practices. The result was similar if the respondent considered the barriers to engaging in safe practices to be too high. For a few respondents, the enablers were strong enough to overcome both of these aspects, and they engaged in protective behaviours despite the barriers. However, it can be particularly challenging promoting taking steps that may have financial and time costs when there is no sign that they are actually keeping people safe. It is this combination of factors that probably accounts for the common opinion by users that there is more they should be doing to protect themselves.

4 A honeypot is an "information system resource whose value lies in the unauthorized or illicit use of that resource." A honeypot can be used for non-malicious purposes in order to prevent attacks by malicious users which that scan the network to identify targets. When the malicious software attacks the system, honeypots can be used to "defend against such attacks by slowing their scanning down, potentially even stopping them." Honeypots used in this way are called 


\subsection{Chapter Summary}

In this chapter, the findings about the factors that influenced respondents' cybersecurity behaviours have been explored. Respondents needed to be aware of the threats before forming opinions about how dangerous they are. Without this awareness, they often considered the danger to be minimal. The perceived danger of the threats was seen as the result of a combination of the perceived likelihood of facing such a threat and the perceived severity of the consequences of the threat. Perceived susceptibility and severity were both influenced by how effective respondents considered their safeguards to be. Awareness and danger were influenced by internal factors such as past experiences with cybersecurity breaches, respondents' knowledge of threats and safeguards, their confidence in their skills, how much they relied on other people to keep them safe, how much they trusted websites and people online, how concerned they were about security, and whether they engaged in ego protective behaviours. Awareness and danger were also influenced by external factors such as symptoms of breaches, the security messages that respondents received, and computer sharing behaviours. Perceived barriers to engaging in security behaviours such as cost and time often diminished respondents' intention to engage in these behaviours while strong feelings about the need for security could increase their intention to protect themselves and their families. Although respondents were often able to identify the things they could do to protect themselves, they still did not. In Chapters 5 and 6, the relationships between these factors are explored in greater detail. In Chapter 7, reasons why people act in unsafe manners despite "knowing better" are discussed. 
Braun 


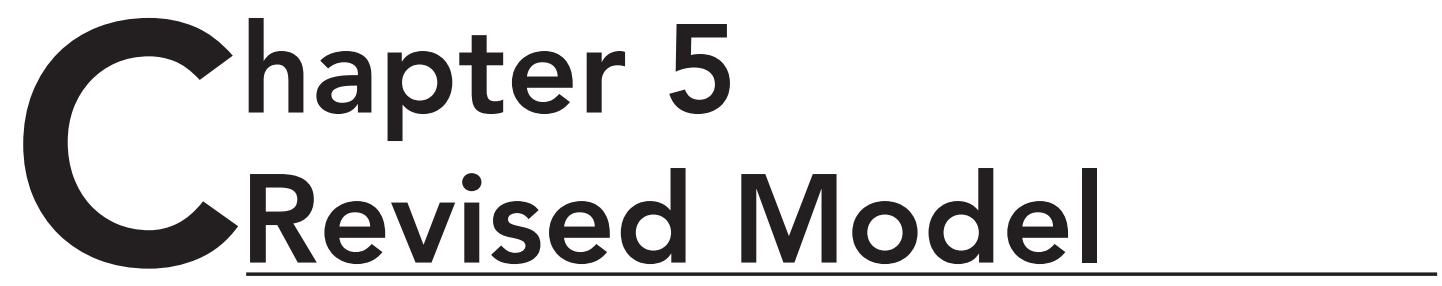

\subsection{Introduction}

In Chapter 4, the factors relating to home users' internet security behaviours were identified, answering research question 1 and addressing research objective 1 . In this chapter, the relationships between the factors are explored and a revised model is presented in order to answer research question 2 which asks how the identified factors influence users' cybersecurity behaviours. Furthermore, this chapter presents a revised model which meets research objective 2 which is to develop a framework to explain the relationships between what users know and what they do. From the conceptual model, the expected driver of cybersecurity behaviours was perceived threat. In the revised model, awareness is identified as a necessary precursor to home users' assessment of the overall danger of the threat. Often previous research appeared to assume the presence of awareness. In this chapter, the discussion of the revised model includes definitions of each of the factors, explores the relationships between the factors, and explains the differences between the conceptual and revised models. 


\subsection{Overview of Conceptual Model}

In Chapter 3, a conceptual model was presented based on the existing home and organisational cybersecurity literature as well as motivational theories. Nine main factors were identified from the literature: perceived threat severity, internal factors, external factors, perceived threat, perceived barriers and benefits, cybersecurity intentions, and cybersecurity behaviours (see Figure 5-3).

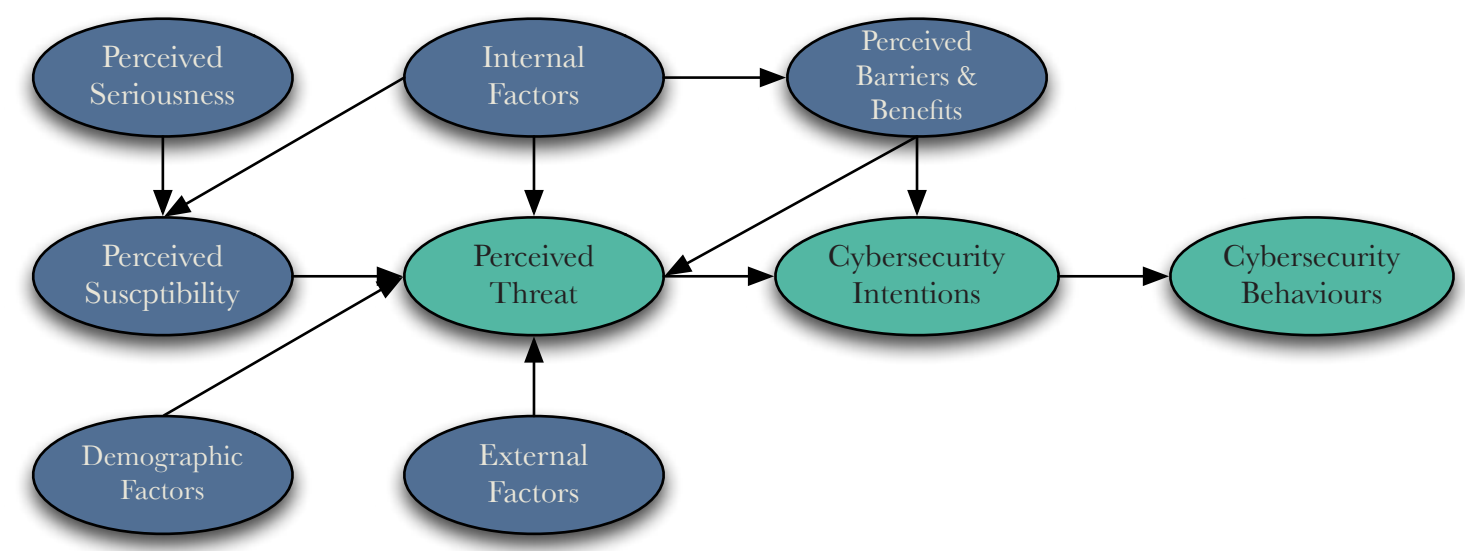

Figure 5-1. Conceptual model used to explore the factors affecting home users' cybersecurity behaviours

The expectation from the literature was that as respondents considered the threat to be more severe, they would feel more susceptible to that threat. These feelings of susceptibility would be further influenced by internal factors (see section 3.3.4) and were expected to lead to an overall perception about the threat. The expectation was that threat perceptions would be influenced by respondents' demographic factors, internal factors, external factors (see section 3.3.5), and the perceived barriers and benefits of engaging in cybersecurity behaviours (see section 3.3.7). This assessment of the perceived threat was then expected to influence respondents' intention to engage in security behaviours, which would then lead to carrying out those behaviours.

\subsection{Revised Model}

After the data had been collected and analysed, the conceptual model was refined and adapted (see Figure 5-2, p. 145). In the revised model, awareness is placed as a precursor to the home user developing a perception about the danger of the threat. Without awareness 


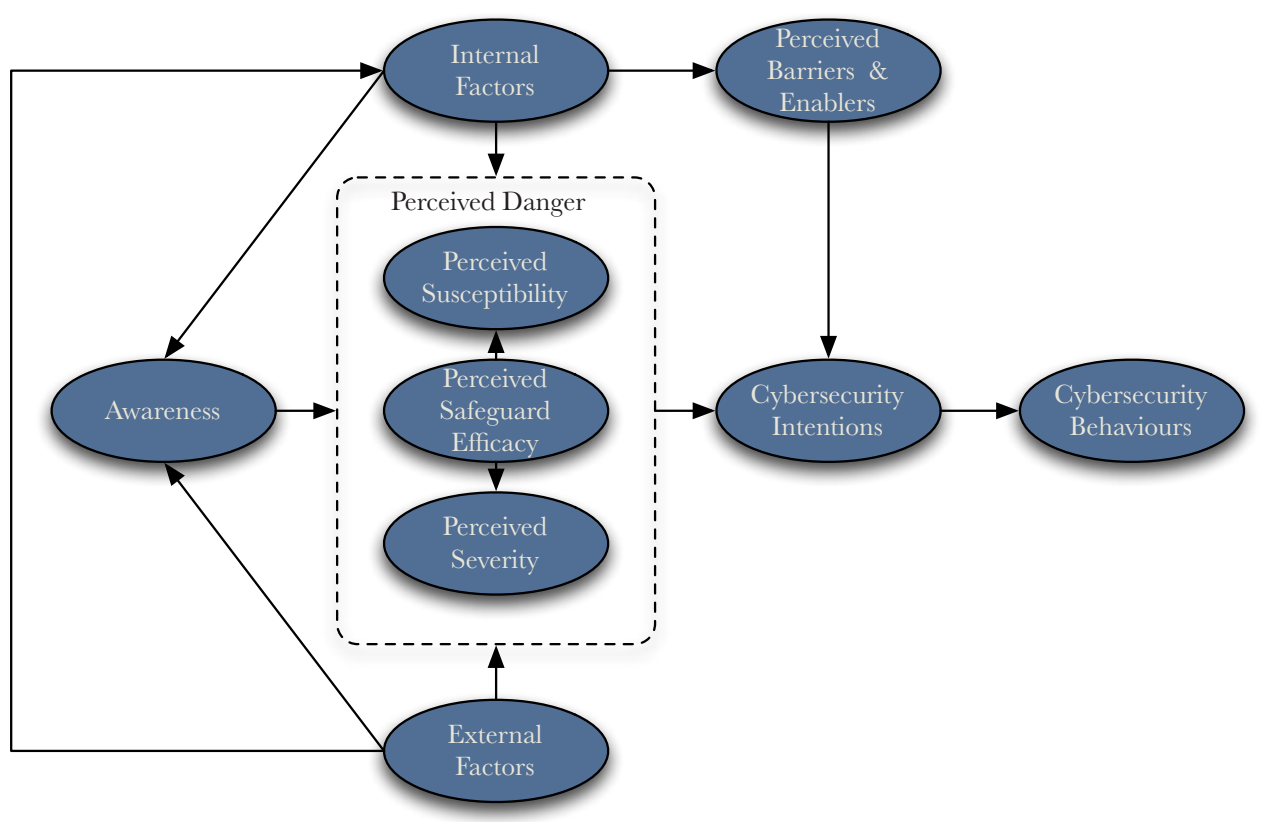

Figure 5-2. Revised model illustrating the factors influencing home users' intentions to engage in cybersecurity behaviours

of some sort of threat, regardless of how specific the threat is, it becomes impossible for there to be an elevated sense of danger. Within the revised model, some factors from the conceptual model were included in higher level factors or were removed entirely. These changes are explained later in the chapter. First, each of the main factors and the components are defined based on the findings from this research.

\subsubsection{Definitions of Revised Model Components}

In this section, definitions for each of the main factors and their components are presented.

\section{AWARENESS}

Awareness is the recognition of the existence of threats online. Although awareness and knowledge are similar, awareness does not require an understanding of the threats, only being conscious of the threat. Awareness was found to be more important than was assumed in the conceptual model. In the original conceptual model, awareness was part of an individual's internal factors. In the revised model, awareness is included as a top level construct. 


\section{Perceived Danger}

Perceived Danger, referred to as "perceived threat" in the conceptual model, is the overall perception of the severity of the consequences of online threats and the likelihood of a user having to face these threats. Within perceived danger, the perceptions of severity and susceptibility are influenced by users' beliefs about the effectiveness of safeguards to mitigate the risks.

Perceived Susceptibility refers to users' perceptions of the likelihood of having to face a threat they are aware of.

Perceived Severity refers to how serious or detrimental users consider the consequences of cybersecurity threats to be. These consequences can be related to the user's computer, data, privacy, or their own sense of wellbeing.

Perceived Safeguard Efficacy refers to how effective users believe that their software, behaviours, and other safeguards will be in preventing security breaches and minimising the consequences of any breach that does occur. The more effective a safeguard is considered to be, the less susceptible users tend to feel they are to threats and the less concerned they tend to be about the consequences.

\section{INTERNAL FACTORS}

Internal Factors refer to the cognitive and affective factors that are unique to the individual. In this study seven factors were identified as internal factors: prior experience, knowledge, perceived self-efficacy, reliance, trust, security orientation, and ego protection.

Prior Experience refers to users' recollections of past experience or lack of experience with cybersecurity threats. For example, if an individual experienced a security breach in the past and was able to remedy it, they may have an increased concern about security breaches as well as a positive view of their ability to cope with issues that may arise. Similarly, if a person does not believe they have experienced a security breach, they may be more prone to feel less vulnerable to threats.

Knowledge refers to the user's understanding of cybersecurity threats. Knowledge is different from awareness as knowledge is associated with understanding what a threat does and how it is propagated, whereas awareness simply requires the user to be conscious of 
the existence of the threat.

Perceived Self-Efficacy refers to users' confidence in their ability to understand threats and to take actions necessary to protect themselves. Perceived self-efficacy is different from knowledge in that perceived self-efficacy focuses on users' having confidence in their abilities and/or knowledge, regardless of their actual abilities and/or knowledge.

Reliance focuses on how much individuals depend on others to (1) prevent security breaches from occurring, and (2) remedying any consequences that occur as the result of a breach. Reliance was not part of the conceptual model, but was added to the revised model as a direct result of the data analysis.

Trust refers to the belief that other people will act in a way that does not compromise the individual's security. Individuals expressed trust in other people, software, organisations, and websites. This trust affected how they interacted with the identified entities. Although trust was seen in much of the literature on adoption of e-commerce, it was not prevalent in cybersecurity research and as a result was not included in the original conceptual model. Based on the data, trust appeared to be more important than originally suggested.

Security orientation refers to how concerned an individual is about their security overall. Security orientation includes feelings they have about the need for and value of security, as well as the actual steps that users take to secure themselves. Security orientation encompasses the concepts of attitude and security orientation from the conceptual model.

Ego protection relates to the presence of behaviours that users exhibit in order to protect the perception that they are safe (typically through boasting) or that they do not need to take steps to be safe (expressed through laziness). Ego protection is new to the revised model. Laziness was associated with respondents considering themselves to be too lazy to protect themselves online while boastfulness was used to highlight behaviours and knowledge that respondents associated with being secure, regardless of the actual benefits of them.

\section{EXTERNAL FACTORS}

External factors refer to cybersecurity related aspects of the individual's environment. These factors include symptoms, security messages, and the context in which the computer is being used.

Symptoms refer to the perceived indications that a security breach has taken place, 
such as data or hardware consequences. These symptoms do not necessarily have to be associated with an actual breach. Instead, what is more important is that the individual believes that the symptoms are indicative of a breach. These symptoms can be either indications of something going wrong on a computer (e.g. a notification from protective software that malware has been identified) or consequences of a breach (e.g. data loss).

Security messages are associated with the influence of communications about online security issues. These messages can include: communication from individuals' workplaces, friends, or family members about potential security issues or how individuals can protect themselves online; news stories about security threats and safeguards' security messages from websites (e.g. banking websites); and other security specific awareness campaigns run by public or private organisations.

Computer Use Context refers to whether or not an individual shares their computer with another member of the household and if they do share a computer, the extent to which the responsibility for the shared computer is assigned to the users of the computer.

\section{Perceived Barriers and Enablers}

Perceived Barriers are the external factors that prevent or limit users from either taking the initial steps to protect themselves online or from taking further steps to increase their protection from threats. The main external barriers identified were the cost of protective software and the time associated with engaging in protective behaviours.

Perceived enablers are the factors that facilitated respondents' cybersecurity behaviours. Perceived enablers were typically related to respondents feelings of security and their desire to ensure that other people using the computer were secure.

\section{Cybersecurity Intentions and Behaviours}

Cybersecurity Intentions refer to users' inclination to protect themselves online through the use of behavioural and software-based safeguards. These intentions are influenced by users' perceptions of the danger of the threats they have identified and their perceptions about the enablers of and barriers to engaging in these behaviours. There is an assumption that if users have a strong enough intention to protect themselves online they will do so. 
Cybersecurity Behaviours are the actual behaviours that users' engage in regarding their online security. These behaviours may be positive (actively protecting the individual's security), neutral (a lack of action in regards to cybersecurity), or negative (actively contributing to increasing an individual's susceptibility to a cybersecurity breach).

A summary of these components is provided in Table 5-1 and on overview of the changes between the conceptual model and revised model is presented in the next section.

Table 5-1. Summary and definitions of the factors in the revised model and the associated components.

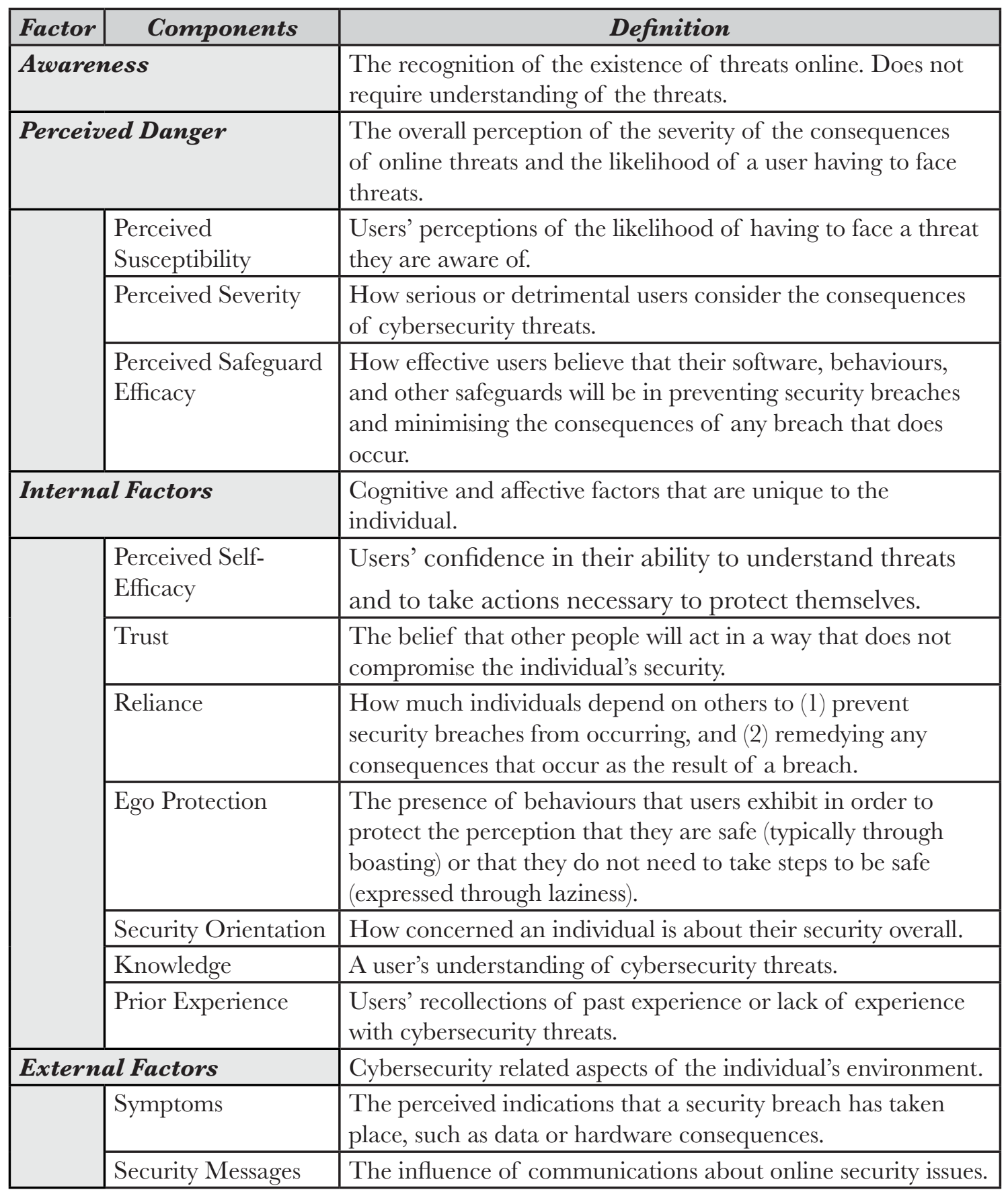


Table 5-1. Continued

\begin{tabular}{|l|l|l|}
\hline Factor & \multicolumn{1}{|c|}{ Components } & \multicolumn{1}{c|}{ Definition } \\
\hline & $\begin{array}{l}\text { Computer Use } \\
\text { Context }\end{array}$ & $\begin{array}{l}\text { Whether the individual shares the computer with another } \\
\text { person in the household. }\end{array}$ \\
\hline $\begin{array}{l}\text { Perceived Barriers \& } \\
\text { Enablers }\end{array}$ & $\begin{array}{l}\text { The factors that discourage or encourage the use of } \\
\text { cybersecurity safeguards. }\end{array}$ \\
\hline \multirow{2}{*}{ Perceived Barriers } & $\begin{array}{l}\text { External factors that prevent or limit users from either taking } \\
\text { the initial steps to protect themselves online or from taking } \\
\text { further steps to increase their protection from threats. }\end{array}$ \\
\cline { 2 - 4 } & Ferceived Enablers & $\begin{array}{l}\text { Users' inclination to protect themselves online through the use } \\
\text { of behavioural and software-based safeguards. }\end{array}$ \\
\hline \multicolumn{2}{|l|}{ Cybersecurity Behaviours } & $\begin{array}{l}\text { Actual behaviours that users' engage in regarding their } \\
\text { online security. }\end{array}$ \\
\hline
\end{tabular}

\subsubsection{Key Changes to the Conceptual Model}

In the development of the revised model, some key changes were made from the conceptual model. The changes to the relationships between factors are summarised in Table 5-2 (p. 151). The greatest change from the conceptual model was that awareness was promoted to one of the main factors. Originally awareness had been accounted for within the concept of knowledge in Internal Factors.

While the concept of Internal Factors from the conceptual model remained in the revised model, there were several changes to the component factors. Within the conceptual model, Internal Factors was comprised of security orientation, perceived self-efficacy, attitude, the subjective norm, prior experience, and knowledge. In the revised model, the subjective norm and attitude were removed, and trust, reliance, and ego protection were added. In addition, the focus of prior experience changed from how long an individual had used the internet to whether they had experienced a perceived cybersecurity breach.

External Factors was previously comprised of cues to action, use by others, and encouragement by others. In the revised model, use by others and encouragement by others were removed due to a lack of support for these concepts. Computer use context was added as an additional component of External Factors. Finally, cues to action was further divided into knowledge, symptoms, and security messages. Symptoms and security messages remained as parts of the External Factors while knowledge was already accounted for within the Internal Factors.

Perceived Susceptibility and Perceived Severity from the conceptual model remained in the revised model, but with some changes. In the HBM (Janz \& Becker, 1984) and PMT 
Table 5-2. Summary of changes to the relationships between the factors in the conceptual model and revised model

\begin{tabular}{|c|c|c|}
\hline $\begin{array}{c}\text { Conceptual } \\
\text { Model } \\
\text { Factors }\end{array}$ & Expected Relationships & Demonstrated Relationships \\
\hline $\begin{array}{l}\text { Perceived } \\
\text { Severity }\end{array}$ & $\begin{array}{l}\text { - Influences perceived } \\
\text { susceptibility }\end{array}$ & $\begin{array}{l}\text { - Influenced by perceived safeguard } \\
\text { efficacy }\end{array}$ \\
\hline $\begin{array}{c}\text { Perceived } \\
\text { Susceptibility }\end{array}$ & $\begin{array}{l}\text { - Influenced by perceived severity } \\
\text { - Influenced by internal factors } \\
\text { - Influences perceived threat }\end{array}$ & $\begin{array}{l}\text { - Influenced by perceived safeguard } \\
\text { efficacy }\end{array}$ \\
\hline $\begin{array}{l}\text { Internal } \\
\text { Factors }\end{array}$ & $\begin{array}{l}\text { - Influences perceived } \\
\text { susceptibility } \\
\text { - Influences perceived threat } \\
\text { - Influences perceived barriers \& } \\
\text { benefits } \\
\end{array}$ & $\begin{array}{l}\text { - Influences awareness } \\
\text { - Influences perceived danger } \\
\text { - Influences perceived barriers \& } \\
\text { enablers } \\
\text { - Influenced by external factors }\end{array}$ \\
\hline $\begin{array}{l}\text { External } \\
\text { Factors }\end{array}$ & - Influences perceived threat & $\begin{array}{l}\text { - Influences awareness } \\
\text { - Influences internal factors } \\
\text { - Influences perceived danger }\end{array}$ \\
\hline $\begin{array}{l}\text { External } \\
\text { Factors }\end{array}$ & - Influences perceived threat & $\begin{array}{l}\text { - Influences awareness } \\
\text { - Influences internal factors } \\
\text { - Influences perceived danger }\end{array}$ \\
\hline $\begin{array}{l}\text { Demographic } \\
\text { Factors }\end{array}$ & - Influences perceived threat & $\cdot \mathrm{n} / \mathrm{a}$ \\
\hline $\begin{array}{c}\text { Perceived } \\
\text { Threat }\end{array}$ & $\begin{array}{l}\text { - Influenced by perceived } \\
\text { susceptibility } \\
\text { - Influenced by internal factors } \\
\text { - Influenced by demographic } \\
\text { factors } \\
\text { - Influenced by external factors } \\
\text { - Influenced by perceived barriers \& } \\
\text { benefits } \\
\text { - Influences cybersecurity } \\
\text { intentions }\end{array}$ & $\begin{array}{l}\text { - Influenced by awareness } \\
\text { - Influenced by internal factors } \\
\text { - Influenced by external factors } \\
\text { - Influences cybersecurity } \\
\text { intentions }\end{array}$ \\
\hline $\begin{array}{c}\text { Perceived } \\
\text { Barriers \& } \\
\text { Benefits }\end{array}$ & $\begin{array}{l}\text { - Influenced by internal factors } \\
\text { - Influences perceived threat } \\
\text { - Influences cybersecurity } \\
\text { intentions }\end{array}$ & $\begin{array}{l}\text { - Influenced by internal factors } \\
\text { - Influences cybersecurity } \\
\text { intentions }\end{array}$ \\
\hline $\begin{array}{l}\text { Cybersecurity } \\
\text { Intentions }\end{array}$ & $\begin{array}{l}\text { - Influenced by perceived barriers \& } \\
\text { benefits } \\
\text { - Influenced by perceived threat } \\
\text { - Influences cybersecurity } \\
\text { behaviours }\end{array}$ & $\begin{array}{l}\text { - Influenced by perceived barriers \& } \\
\text { enablers } \\
\text { - Influenced by perceived danger } \\
\text { - Influences cybersecurity } \\
\text { behaviours }\end{array}$ \\
\hline $\begin{array}{l}\text { Cybersecurity } \\
\text { Behaviours }\end{array}$ & $\begin{array}{l}\text { - Influenced by cybersecurity } \\
\text { intentions }\end{array}$ & $\begin{array}{l}\text { - Influenced by cybersecurity } \\
\text { intentions }\end{array}$ \\
\hline
\end{tabular}

(Plotnikoff \& Higginbotham, 2002) these factors were considered together. Other studies such as Ng, et al. (2008), Johnston and Warkentin (2010), and Liang and Xue (2010) consider them separately. However, there is support for looking at these factors as part of 
the threat appraisal process from Neuworth, et al. (2000), Woon, et al. (2005), and Liang and Xue (2009). From the evidence in this study, respondents considered their susceptibility to threats and the severity of consequences as part of their overall appraisal of the danger of the threats. The result was that these factors as well as the perceived efficacy of safeguards led to a renamed factor called Perceived Danger.

Demographic Factors from the conceptual model were removed in the revised model. It was expected that the demographic factors would have a greater effect than the evidence showed. While demographic factors did influence respondents' information seeking behaviours and their levels of reliance, they did not have a wider effect. As a result, they were removed from the revised model.

The remaining factors of Perceived Barriers and Benefits, Cybersecurity Intentions, and Cybersecurity Behaviours had few changes. The main change was that Perceived Barriers and Benefits was renamed as Perceived Barriers and Enablers to better reflect the difference between the effectiveness of safeguards (as benefits were defined in the HBM), and the positive factors that encouraged individuals to engage in safer behaviours online. Cybersecurity Intentions and Cybersecurity Behaviours remained the same as they were in the conceptual model.

\subsection{Relationships between Identified Factors}

In the revised model (Figure 5-2, p. 145), awareness was positioned as a prerequisite to individuals forming opinions about the perceived danger of threats. External factors were found to influence certain internal factors along with the awareness of threats and perceptions of danger. Internal factors also influence awareness and perceptions of danger, as well as perceived barriers and enablers. Finally, perceived danger along with perceived barriers and enablers influence intention to engage in cybersecurity behaviours. The relationships between the constructs along with the relationships between relevant factors are explored in the subsequent sections. Changes from the conceptual model are also highlighted in the appropriate sections.

\subsubsection{Influence of Awareness}

In the conceptual model, awareness was included as one of the internal factors. However, based on the data, awareness was removed from the internal factors and promoted to one of the main factors influencing home users' cybersecurity behaviours. From the data it was possible to see that awareness underpinned many of the feelings held by respondents about severity, susceptibility, and the overall danger of threats. 
Within the current study, respondents often commented on how they were unaware of where to start when addressing cybersecurity beyond the basic step of having antivirus software. One of the interviewees elaborated on why she did not do more to protect herself and indicated:

I don't have any knowledge about [other safeguards]. I only [know about] that particular antivirus software... (Int-12)

Comments such as this and other comments about respondents not knowing where to start in regards to their security showed a lack of awareness about safeguards. The results further showed that in order to form opinions about how susceptible to threats respondents were and how severe the consequences were, they first needed to be aware of the threats and the steps that they could take to avoid them.

The effect of awareness on cybersecurity behaviours has only begun to be explored in recent years. Although Drennan, et al. (2006) found that awareness was not linked to awareness in respect to privacy, this study as well as one by Johnson \& Warkentin (2010) suggest that a link may be present in the broader security context. Supporting the idea of placing awareness as a precursor to perceived danger, Johnston \& Warkentin (2010) used Witte's (1992) definition of threat to explore awareness and stated:

A threat is an external stimulus that exists whether or not it is perceived by an individual. If an individual perceives the threat, that individual can be described as having awareness of a threat.... once an individual is conscious of a threat, he or she will establish beliefs as to the seriousness of the threat and probability of personally experiencing the threat. (p. 551)

However, Johnston \& Warkentin did not explore the value of awareness or what helps to create awareness in order to develop these beliefs about the danger of threats. Instead they focused on the severity and susceptibility aspects within fear appeals.

\subsubsection{Perceived Danger}

In the conceptual model, this factor was originally named "perceived threat" based on the term used in the HBM (Figure 2-2, p. 32). Originally, perceived threat was defined as "the threat posed by illness, comprised of the likelihood of its occurrence ("perceived susceptibility') and it's potential for causing physical harm and interfering with social functioning ('perceived severity')" (Becker et al., 1977, p. 349).

Respondent awareness of threats tended to influence how dangerous they thought 
the threats were. When respondents were unaware of threats, they were often unable to see the actual danger of a breach or what the potential consequences were. However, awareness of threats was not necessarily straightforward in the context of security. There appeared to be a difference between how aware respondents considered themselves to be of threats compared to how aware they actually were of threats. If their perceived awareness of threats was higher than their actual awareness of threats they were more likely to make incorrect assumptions about how well they were protected.

In some cases, respondents considered themselves to be highly aware of threats because of the information they obtained at work. This often led to the belief that they were well protected at home because of that awareness. However, this assumption could be dangerous. While talking about why the average user considered themselves to be aware of threats, an interviewee noted:

I think part of people being really sloppy about home security is because they've got such good security at work, that it gives them this false sense of security. It's all hidden at work. You never have to click any antivirus stuff, you never have to do anything. You just sort of [pause] it's all done for you in the background. Whereas at home, you kind of think somehow that might just happen by magic. You know, you don't have the technical skills necessary to [protect yourself]. (Int-13)

The danger here was that respondents at times assumed that their awareness of threats was enough to protect them. Although in the quote above the respondent noted how work experience could influence individuals' security behaviours, other respondents typified the example given. Other respondents discussed how the information they had gained at work made them more aware of threats. Consequentially, respondents who considered themselves to be highly aware of threats often considered themselves to be less susceptible these threats and thus, the perceived danger of threats to be low to moderate. When these perceptions of susceptibility were looked at in conjunction with the actual steps that respondents took, there were times where perceived awareness was high but the safeguards that respondents reported using were minimal. This suggested that awareness on it's own was not enough to influence protective behaviours. However, it also shows how awareness can decrease the perceived danger of threats which contributed to a reduced intention of engaging in protective behaviours. 


\subsubsection{Internal Factors}

The internal factors construct from the conceptual model remained in the revised model. However, the components differed from what were originally expected on the basis of the literature. The subjective norm did not appear to have an influence on respondents' intentions to engage in security behaviours and therefore was removed from the revised model. Similarly, attitude was encompassed by the component of general security orientation. New components of reliance, trust, and ego protection were added to the revised model. What emerged was that these internal factors did not act in isolation to influence awareness, perceived danger, and perceived barriers and enablers. Instead, they also influenced each other.

\subsubsection{Relationships between Internal Factors}

From the data it was possible to see how the internal factors related to each other and influenced each other in both positive and negative ways. Figure 5-3 illustrates these

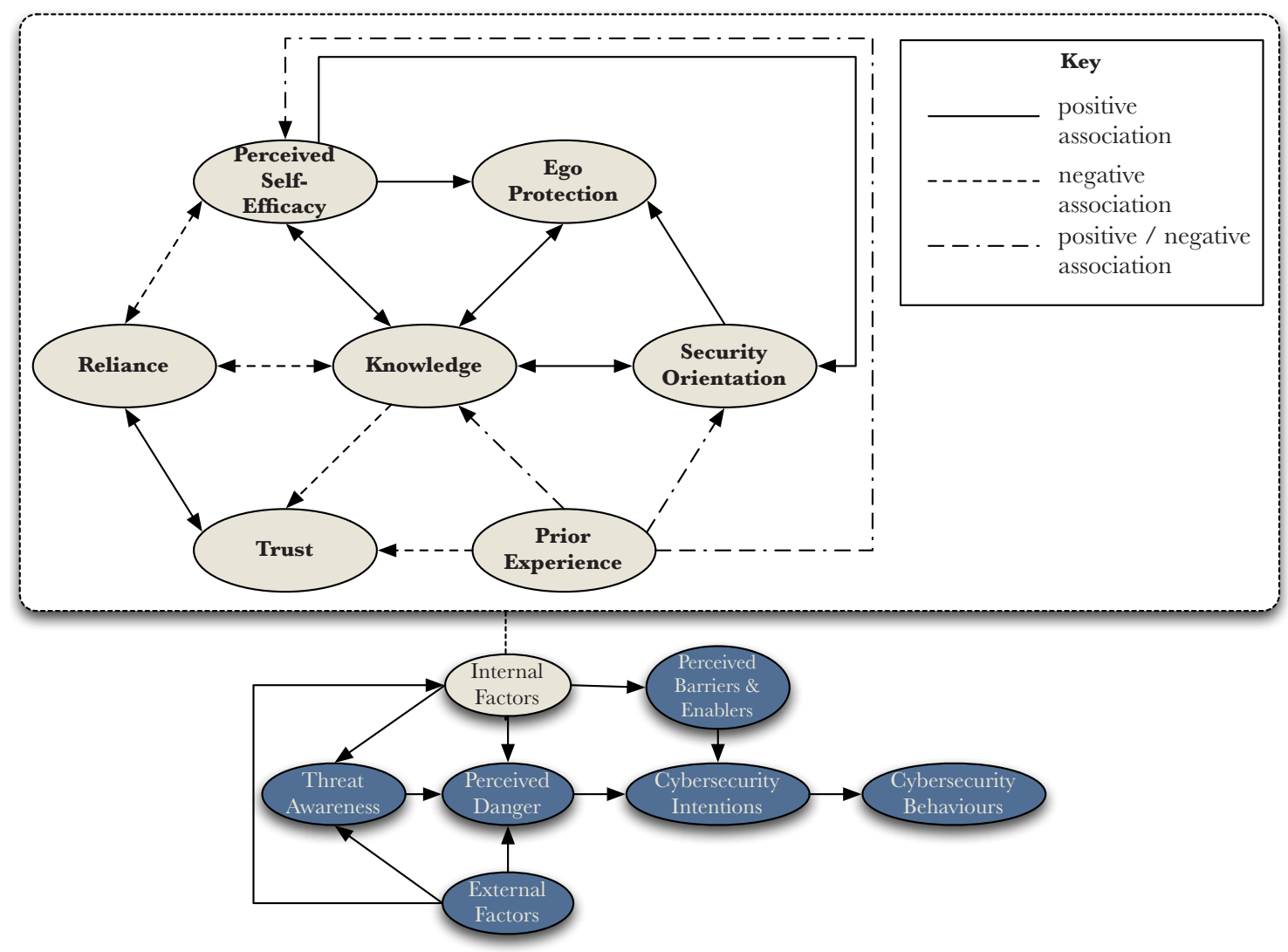

Figure 5-3. Relationship between components of internal factors relating to home users' internet security behaviours 
influences and the types of relationships that the factors have on each other. At the centre of the internal factors is knowledge, which appears to have the greatest influence on most of the other internal factors and in turn is influenced by these factors as well.

Security orientation has a positive influence on knowledge. Respondents who were concerned about their security typically were more likely to seek out information to increase their knowledge in order to help alleviate some of their concern. Similarly, knowledge also had a positive influence on security orientation. The more knowledgeable a respondent was about threats online, the more concerned they tended to be about their security. One particularly knowledgeable participant noted how he used many different identities online because of an increased concern about security:

I use different passwords. My wife uses the same passwords for everything. I use different passwords for each and every place, and I spell my name differently. So when I send emails out, I don't use the same name when I do things for [my business] as I do when looking at things like getting information for travel. (Par-2E)

By using the different identities he was able to determine which sites had "sold" his information. Knowing this helped alleviate his concern because he knew which sites were safe and which were distributing his information. Because of this, he was able to focus his concern on the sites that were not protecting his information. Respondents with lower levels of knowledge and security orientation were more likely to emphasise that they knew better but were simply too lazy to protect themselves. This allowed them to still project the image that they were concerned about security even if other comments suggested that their concern was relatively low.

Prior experience had a positive influence on knowledge if the respondent had experienced a cybersecurity breach. Often respondents indicated that the symptoms that they experienced and the consequences of those symptoms made them seek out more information, either on how to remedy a breach or how to avoid one occurring in the future. One of the interviewees discussed how she had discovered information about the symptoms her computer was exhibiting:

I'll search for what's happening. And usually that will lead me to some potential ideas of something more specific, and then I really just keep getting more and more specific until I answer my question. (Int-7)

By searching out this information, she believed that she had gained more knowledge not only about the specific problem, but more knowledge in general about how to avoid and remedy computer security issues. As a result, the past experiences with cybersecurity 
breaches led to her gaining additional knowledge. Good, et al. (2006) identified two types of users who were impacted by their prior experience. Individuals who "install first, ask questions later" were confident in their ability to remove programs because of their past history with installing programs. Individuals who where "once bitten, twice shy" were more knowledgeable about past risks because of their previous negative experiences. In the current study, the beneficial effect of past experience on knowledge was greatest when respondents had enough confidence in their abilities to try to remedy an issue themselves.

A lack of prior experience also had an effect on knowledge because it usually meant that respondents did not see the need to increase their knowledge. Instead, they typically preferred to believe that the knowledge they already had was sufficient to protect them, since it had done so in the past:

Nothing major has ever happened to any of us yet. So it's kind of like, well, we've made it this long, so what's the point. (Par-2B)

Similar comments by respondents often indicated that if they had not noticed that something had gone wrong with their computer they assumed that everything was fine and the computer had not been compromised. However, this also showed an assumption by respondents that all security breaches would have clear, tangible symptoms.

Respondents with higher levels of knowledge tended to be less reliant on others, while respondents with lower levels of knowledge were more reliant on others. In many cases, respondents with lower levels of knowledge were less certain about who was responsible for their online security, and as a result were more likely to push the actual responsibility onto someone else. For example, one of the older participants stated:

[The responsibility for our computer's security] is shifted to my husband or children who know about these things. (Par-3B)

Although she indicated a reliance on her husband who she assumed was more knowledgeable, she also implied that age was related to knowledge that a person had about the internet and online security with her mention of her children. This assumption was echoed throughout the discussions, primarily by people who had children that they could rely on to provide them with the relevant knowledge. There tended to be an assumption that because their children had grown up using the internet and computers, they knew more about being safe online regardless of their actual knowledge There also was an implied assumption in comments similar to the one above that whoever the respondent considered to be responsible for the computer was not only more knowledgeable than they were, but that they would also take responsibility for protecting the computer. 
Just as respondents who were less knowledgeable set out to rely on someone else to protect their computers, respondents who relied heavily on others often did not see the need to increase their knowledge. For example:

I would get [information] from the people [at work], which I might say to [my husband] "have you heard about this" and he usually would have, and usually would be doing something about it at home. I personally wouldn't be doing anything. (Int-5)

In this case and in similar situations, respondents who relied heavily on other people to protect their computers typically did not take steps beyond basic awareness, and instead relied on the other person to have the required knowledge to ensure that the computer was kept safe. Similar behaviours were identified by Albrechtsen, et al. (2007) within an organisational environment where individuals who did not have security responsibilities in their job were more likely to rely on the IT department to remedy any issues.

Conversely, respondents who did not rely on others felt that they needed to have more knowledge since there was nobody else they could depend on to have that knowledge.

Me, I should be responsible for [my security]. Because first of all, you cannot control how many viruses are around there and how people actually could, you know, take any sort of extra steps to protect their security. Let alone infecting others. So, you know, so, you know I'm not trying to save the world, I want to save my stuff, so I need to take my responsibility. (Int-11)

Respondents with higher levels of knowledge were much more likely to accept responsibility for their own actions. They were much less likely to try to transfer responsibility for breaches overall, often only blaming themselves.

Respondents with high levels of perceived self-efficacy were often more confident in their ability to understand the information they were presented with. In contrast, with some respondents, a lower level of perceived self-efficacy often was a stumbling block to their seeking out more knowledge. They often made comments such as "I wouldn't know where to start." Although these comments indicate a certain lack of knowledge, they also indicate a lack of confidence among respondents about their ability to take the necessary steps to protect themselves. Bandura (1977) indicated that people with lower levels of self-efficacy were more likely to blame failures on a lack of knowledge (p. 198). The lack of knowledge demonstrated by respondents in the current study was linked to their lack of confidence, which in turn related back to seeking out someone else to protect their computer. This in turn raised issues with respondents feeling like it was their responsibility to increase their 
knowledge.

In some cases, there were large differences between respondents' perceived selfefficacy and their demonstrated knowledge. In such cases, their behaviours appeared to be influenced more by their perceived self-efficacy. Knowledge and perceived self-efficacy appeared to have an almost circular relationship. In some cases, people who did not consider themselves able to understand the information they could find on the topic, preferred to avoid seeking out additional information that might make them more knowledgeable. In turn, by increasing their knowledge they would have increased their confidence in their ability to protect themselves. However, because they did not have that confidence in their abilities in the first instance, they did not seek out additional information.

In many cases, ego protection was used as a way to distract from a lack of knowledge. Boastfulness allowed respondents to present a façade of confidence, typically focusing on their awareness of threats, in order to distract from the lack of a deeper understanding of what the threat could do or how they could protect themselves from it. Rose, Endo, Windschitl, and Suls (2008) found that unrealistic optimism was common amongst cultures, offering a partial explanation for why individuals were likely to engage in unrealistically positive assessments of their security in the current study. Laziness was also used to distract from a lack of knowledge by putting the focus on a perceived lack of need for security. Respondents who considered themselves lazy and demonstrated lower levels of knowledge often implied that there was no need to take steps to protect themselves. This was underscored by numerous respondents noting that they knew what they should do to protect themselves but that they simply did not take those steps. Although respondents' first inclinations were to suggest that they were lazy about security, further questions suggested that this laziness was often prompted by a lack of concern about security. This lack of concern tended to stem from a combination of low levels of perceived susceptibility, perceived severity, knowledge, and awareness.

Finally, respondents who were more knowledgeable were generally less trusting of people and sites online. This stemmed from an increased understanding of what could go wrong if they trusted people online. People who had greater knowledge were more likely to be able to explain what the consequences of breaches were, and as a result, they understood that malicious users' actions were typically malicious rather than benign. This led to very low levels of trust by respondents with high levels of knowledge.

The effect of prior experiences on perceived self-efficacy could be both positive or negative. An instance of a positive outcome from a security breach was that the person was able to fix the problem by themselves or that they felt they had in some way decreased the severity of the consequences. Bandura (1977) found that success at a task was key to developing self-efficacy (p. 195). For example, an interviewee commented: 
I've made the mistake of visiting dodgy sites, and then I'd wind up having to reinstall Windows because I'd be plagued with popups and things like that. And that was my own fault. And I know back when I was using a [Windows] PC, I'd occasionally get my antivirus software catching things too. (Int-7)

In this case, although she had experienced security breaches, the interviewee had taken steps that she considered sufficient to fix the problem and was able to return her computer to a safer state. She later indicated that she felt that these previous experiences had helped make her more confident in her ability to protect herself online.

In contrast, respondents who had experienced very negative consequences of security breaches indicated that at times this had made them less confident in their abilities. Failures at accomplishing a task early on have been linked to lower levels of perceived self-efficacy (Bandura, 1977). One of the interviewees described how her past experiences had made her feel:

When I was [a student] back in school, one of the students hacked into my email account and sent a couple of nasty ones around. And oh my god! Like, you've, you just can't [pause] Like I spent a couple of weeks dealing with that. (Int-2)

Although this particular interviewee engaged in very safe behaviours and demonstrated high levels of knowledge, her perceived self-efficacy was much lower than other users who demonstrated similarly high levels of knowledge. In cases such as this, the main distinguishing factor was that the respondents had experienced what they considered to be very negative consequences associated with security breaches. At times, past experience was the defining feature that made individuals more or less confident about their security skills. This shows how it is important to not only consider whether or not an individual experienced a breach, but also how severe they considered the consequences to be. The more negative the perceived consequences, the more often it had far reaching consequences into how the respondent perceived their security with past experiences having the potential to influence users' behaviours years after the original breach occurred.

Lower levels of perceived self-efficacy were associated with higher levels of reliance on others. Respondents with lower levels of perceived self-efficacy often made comments such as "I wouldn't know where to start." Although these comments also indicate a lack of knowledge, they also indicate a lack of confidence in the respondents' ability to protect themselves online. In many cases, these respondents sought out someone they considered to be more skilled and knowledgeable than themselves to protect their security. Often they relied on a family member living in the same household to protect the computer. 
In contrast, respondents with higher levels of perceived self-efficacy felt that they could protect themselves online. They also considered themselves to be more skilled at doing so than their peers. This meant that they were less likely to rely on someone else because they felt that other people could not help to make them more secure. When one of the interviewees discussed why he did not expect to get help from other people, he stated:

I think that people around me are less aware of the security threats... So basically, I think that I, you know, I mainly trust what I have known about because people around me don't really know what's going on. So I don't really listen to them. (Int-18)

Respondents who had higher levels of perceived self-efficacy but lower levels of actual knowledge had more moderate levels of reliance.

Perceived self-efficacy also influenced security orientation. However, in order to be influential, there needed to be high levels of both perceived self-efficacy and knowledge. If there were lower levels of knowledge and higher levels of perceived self-efficacy, respondents were not as likely to be concerned about their security, instead focusing on reasons why they did not need to be concerned. Aytes and Connolly (2004) had a similar finding, and showed that although their respondents considered themselves to be knowledgeable about security threats due to awareness campaigns, this awareness did not translate into action.

Respondents with higher levels of perceived self-efficacy, especially those who also had low levels of knowledge, were more likely to engage in ego protective behaviours. This was often because they wanted to distract from their lack of knowledge while still preserving the façade of confidence that they wanted to project.

In order to rely on a person to protect a computer, respondents needed to not only trust that the person was acting in their best interest, but also that there were actually acting to protect their security. For example, an interviewee indicated that her trust in people to protect her allowed her to rely on them to do so:

I feel reasonably secure because I know that someone else both at work and at home is doing the job for me that I trust. (Int-13)

In this case, there was an implication that if she did not trust the people who were acting to protect her security, she would not have felt as secure in relying on them. Thus, a certain level of trust was required for individuals to be reliant on others. Without that trust, they were less likely to rely on others to protect their cybersecurity.

Respondents who had experienced the negative consequences of a security breach in the past were typically less trusting of people and websites online than those who had not. 
For example, a participant who had experienced several security breaches in the past stated:

I'm not necessarily paranoid, but [because of my past experiences]... I have a different attitude about [security]. $($ Par-2E)

He went on to talk about how he was extremely distrustful of people online, even going so far as to avoid using the computer for anything more than the most basic tasks. In contrast, respondents who had not experienced a security breach were more willing to give people online the benefit of the doubt and to consider people to be trustworthy until proven otherwise. Respondents who had experienced security breaches considered themselves to be much less trusting of people overall because they had already experienced breaches and therefore, had greater levels of concern, similar to Good, et al.'s (2006) user type of "once bitten, twice shy". Most respondents who had experienced very negative consequences of breaches also had low levels of trust. However, some respondents who had experienced what they considered to be less serious consequences had more moderate levels of trust. As a result it appeared that trust, while influenced by the presence of past negative experiences, was primarily influenced by how severe the respondents considered the consequences to be.

Past experiences were also linked to security orientation. a participant spoke about how his prior experience with cybersecurity breaches made him much more concerned about security:

I've already lost one computer that locked up one time. And I got infected twice already. (Par-2E)

When respondents believed that they had experienced a cybersecurity breach, the protective steps they were taking tended to be more proactive than those who had not experienced a security breach. Those participants who had experienced a breach indicated that they were more worried about the possibility of it happening to them again. Furthermore, respondents who had experienced cybersecurity breaches in the past tended to take more protective steps overall than people who had not experienced breaches.

\subsubsection{Internal Factors and Awareness}

Prior experience, knowledge, and security orientation had the greatest positive influence on respondents' awareness (see Figure 5-4, p. 163). Respondents with higher levels of these three factors had greater awareness than respondents with lower levels of these factors. Higher levels of reliance and trust had a more negative effect on awareness. Perceived self-efficacy were both positively and negatively associated with awareness (see 
Chapter 5 : Revised Model

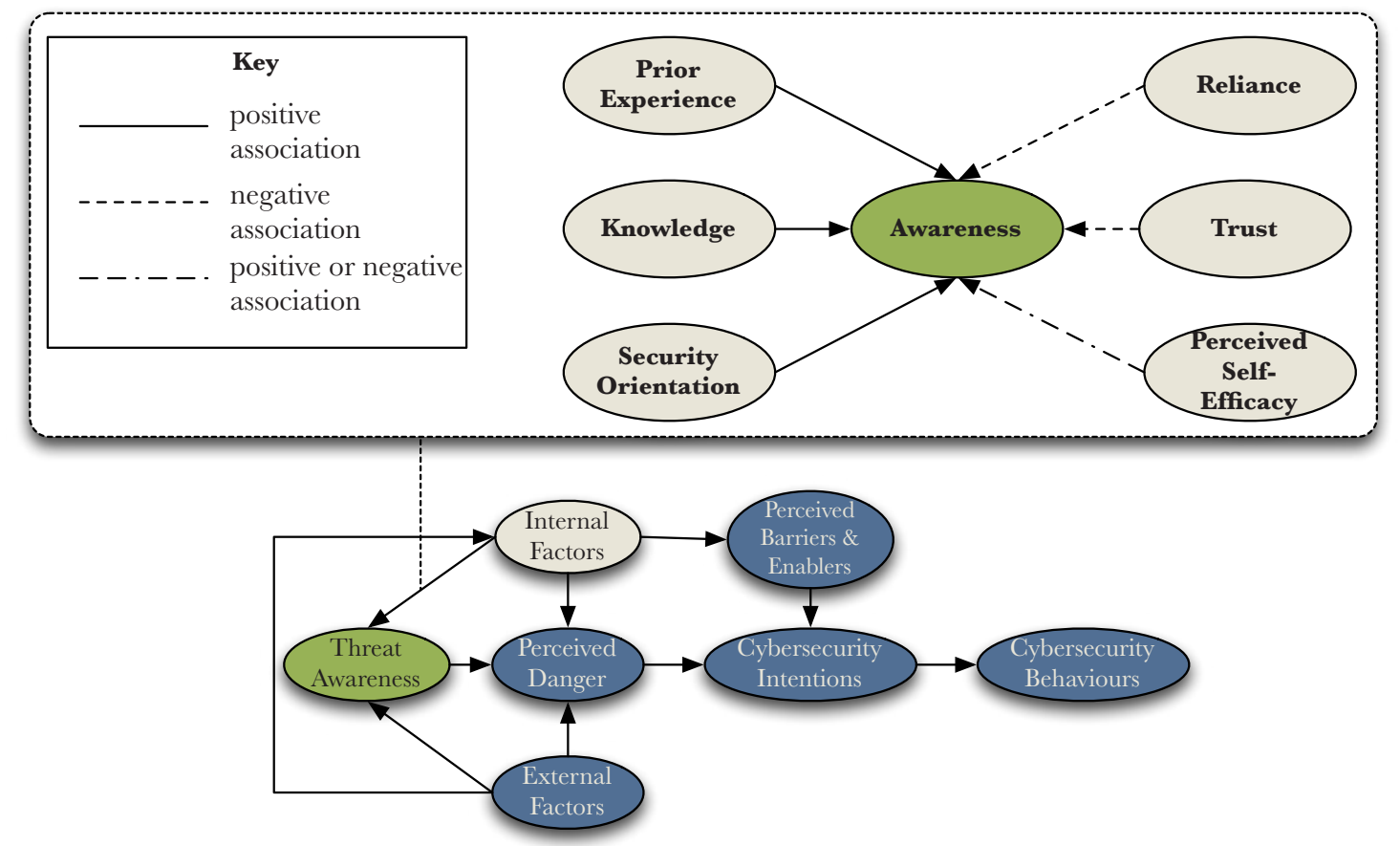

Figure 5-4. Influence of components of internal factors on awareness

Figure 5-4). When respondents had experienced consequences of breaches often increased awareness because it helped to trigger the need to be aware at least once in the past. These negative experiences had a positive effect on respondents' awareness. For example, when a participant talked about why he considered himself to be aware of threats, he noted how the fact that he had "lost everything" had contributed to increasing his awareness. Similar comments made by respondents illustrated how prior experience, especially when the consequences were very negative, had a positive effect on respondents' awareness of threats. In contrast, respondents who had not experienced consequences of a cybersecurity breach often felt that because "nothing had happened [to them] so far", there was little need for increased awareness. They questioned the importance of security overall and indicated that they were more likely to be reactive rather than proactive about their security.

The more knowledge respondents had, the more aware they were of threats. Knowledge and awareness were closely linked because in order to gain more information about threats, respondents first needed to be aware of them. If they were unaware of threats, they lacked the necessary starting point for increasing their knowledge. Respondents who had lower levels of knowledge about threats were less able to identify specific threats. Instead, they described them in very broad terms such as "malware" and "spam" and were unable to elaborate on these threats. They also tended to include consequences of threats rather than threats when identifying the threats they were aware of, such as "identity fraud." Albrechtsen (2007) found that the less that individuals understood the threats and consequences, the less likely they were to be concerned about the danger of security threats.

The greater respondents' security orientation, the greater their concern about threats. 
This resulted in higher levels of awareness because of the preexisting concern. Respondents who were more concerned about threats were more likely to seek out information, which often resulted in greater awareness. Information seeking has been linked to greater levels of awareness (see, Wang, Xiao, and Rao, 2010). An example of the link between information seeking behaviours and awareness illustrated through a respondent's comment about how he actively sought out information when he was explaining why he considered himself to be aware of threats:

I read quite a few technical articles and also read the news and things like that, keep myself updated in terms of what is the threat and what are the latest issues in terms of security and privacy. (Int-11)

Similar comments by respondents about how they kept themselves up to date with security issues were made when talking about their awareness of threats. This suggested that increased concern led to increased information seeking behaviours, which in turn led to increased awareness of threats. Similarly, if respondents were already unconcerned about threats, they were less likely to take note of information about security threats and were less likely to seek out information themselves. This seems to be due in part to a perception that the security threat is not relevant to the individual (for example, see Blanton, et al., 2001; Furnell, Bryant, and Phippen, 2007; Johnston \& Warkentin, 2010). Respondents who felt that threats were not relevant to them typically had lower levels of awareness. For example, one of the respondents with lower levels of awareness stated:

I'm probably less informed and I don't usually pay attention to what's going on with various threats (Par-3A)

These types of comments were often made by respondents when explaining why they did not consider themselves to be aware of threats. Often they believed that in order to be aware of threats, you had to pay attention to them, which required an existing level of concern about threats.

Higher levels of reliance were associated with lower levels of awareness because respondents felt that someone else would be aware of any threats that they needed to be concerned about. One of the stumbling blocks to good user behaviours has been identified as security not being perceived as the responsibility of the individual in question (Albrechtsen, 2007). Typically the more reliant respondents were, the less responsibility they took for knowing what the threats were or how they could protect themselves. Trust also tended to have a negative influence on awareness because respondents believed that people were generally trustworthy, therefore there was no need to be concerned about threats and no need to be aware of what the threats were. 
Finally, perceived self-efficacy had both positive and negative effects on awareness. High levels of perceived self-efficacy often resulted in high levels of awareness because of the existing knowledge about threats. However, high levels of perceived self-efficacy could be associated with lower levels of awareness if combined with lower levels of knowledge because respondents assumed that they were already aware of threats, even if that information was out of date or incomplete.

\subsubsection{Internal Factors and Perceived Danger}

Security orientation, knowledge, and prior experience were positively associated with perceived susceptibility (see Figure 5-5). As respondents' levels of these factors increased, so did their perceptions of danger. While security orientation and knowledge both also had a positive association with perceived severity, the effect of prior experience was mixed. The effect of prior experience on severity depended more on the type of consequences that the person had experienced in the past and whether they considered these consequences to be severe. This often meant that respondents who had experienced particularly negative consequences in the past were more likely to consider the severity of future threats to be just as bad. In contrast, respondents who had been able to remedy the consequences of breaches in the past or who considered the consequences to be minor were more likely to

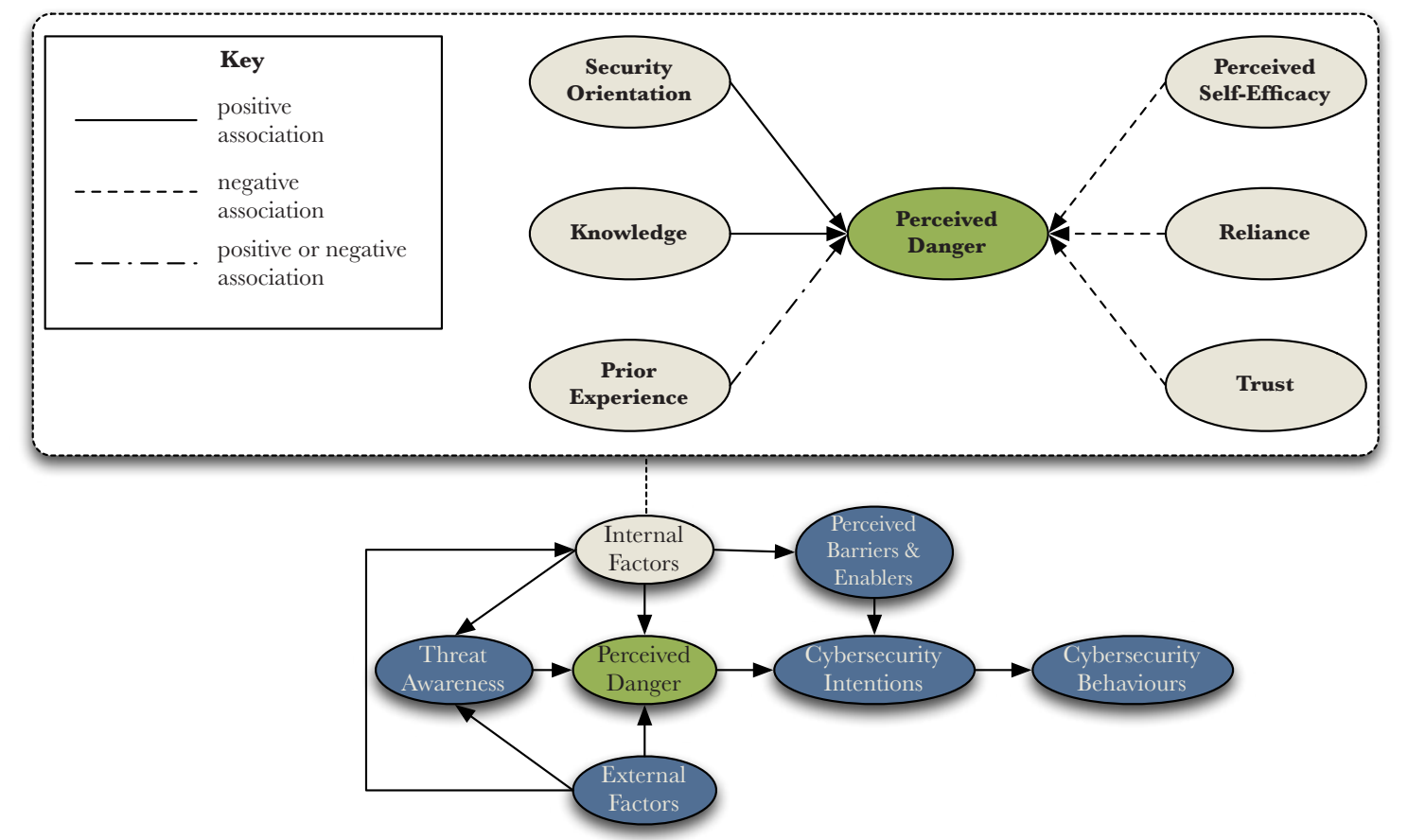

Figure 5-5. Influence of components of internal factors on perceived danger 
consider the severity of a future breach to be lower.

Respondents with higher levels of security orientation tended to describe themselves as "paranoid" about the likelihood of suffering from a cybersecurity breach. They often indicated that if a malicious user wanted to target them, there was "little that could be done" to stop it. Similarly, when talking about the severity of consequences, respondents who had higher levels of security orientation and expressed concern about their susceptibility to malicious users, they often spoke of how "devastating" the consequences would be in terms of their feelings of security. Similar statements were not made by respondents with lower levels of security orientation as they typically viewed the severity of the consequences to be much milder. Past research has linked security orientation to severity in regard to intention to engage in online security behaviours (see $\mathrm{Ng}$, Kankanhalli, \& Xu, 2008).

Higher levels of knowledge often resulted in higher levels of perceived susceptibility and perceived severity. Often respondents with higher levels of knowledge felt that they thoroughly understood the dangers and knew more about the steps that malicious users could take to breach their systems. This led to greater feelings of susceptibility. Similarly, because they knew what the potential consequences were, they tended to view them as being much more severe. For example:

I know what sort of damage it could do. Like delete your hard disk or corrupt all your files, or make them inaccessible... I mean, I understand that it could completely melt down my laptop... It can do a lot of damage. (Int-14)

Unlike respondents with lower levels of knowledge who often viewed the consequences as being more of annoyances, respondents with higher levels of knowledge were typically able to elaborate on the specific consequences they were concerned about. Furthermore, this understanding of the consequences resulted in a greater fear of what could happen.

When respondents had experienced negative consequences in the past, they tended to feel more susceptible to threats. This often meant that they took additional steps to try to mitigate their concerns over the potential consequences, in order to reduce their perceptions of susceptibility:

[My past experience] would have made me make sure that I purchased the antivirus software and made sure that it's actually running and that it's connected to the internet and when it said "please download me now" I did it. And stuff like that. (Int-14)

Because this interviewee had already experienced something similar in the past, she felt that it was likely to happen again, which made her want to take additional steps to avoid the situation from re-occurring. Comments such as this were common amongst 
respondents who had experienced breaches in the past because they wanted to avoid feeling as susceptible as they would without additional protective steps being taken. This appeared to also be influenced by how severe they believed the consequences would be based on their previous experiences:

I mean, in some ways the... very first virus that we got on our, my old computer kind of was kind of scary so I've always tried to be as secure as I can. (Int-15)

This interviewee viewed the consequences of threats to be much more severe because of the fear that he had experienced as a result of an earlier breach. While respondents who had negative experiences tended to believe that future consequences would be just as severe, those who had less negative experiences were generally less concerned about the severity of the consequences. Past experience has been shown as influential in relation to perceived susceptibility to consequences (see Johnston \& Warkentin, 2010). Overall, the consequences of the breaches respondents experienced dictated whether their prior experience had a positive or negative effect on their perceptions of severity (see also Chen \& Bansal, 2010).

Perceived self-efficacy, reliance, and trust, all had negative associations with both perceived severity and perceived susceptibility (see Figure 5-5, p. 165). As respondents' levels of these factors increased, their perceptions of the danger of threats tended to decrease. As respondents' perceptions of self-efficacy increased, they tended to talk more about the steps that they took to protect themselves online. A participant talked about the various steps that he took to ensure that he was less susceptible to threats.

I got Windows firewall up, Norton anti-virus, Spybot, and Lavasoft Adaware for programs. Then I never open attachments, the only emails I get are emails from her or ones I send home from work. Watch what websites I go to. (Par-2A)

From his experience with computers he had gained a great deal of confidence in his ability to not only avoid threats, but also to remedy them. As a result, he felt that the consequences would be less severe because he could fix them.

I feel pretty secure, check my bank account daily at work, so if something did occur, credit card companies will reimburse you if you file right away, which I would, so I feel very confident that if something did occur, I could fix it. (Par-2A)

Comments about respondents' ability to fix problems were often made when they were explaining why they felt that the consequences would be less severe, despite the fact that they were still concerned about breaches happening: 
Yeah, of course I'm concerned about financial loss and identity theft and things like that. And while that would be horrible, part of me thinks: well, I know I would have to go through a lot to get back to square one, but it could be fixed. (Int-7)

Similar respondents felt that they were safer because of their skills, which they were highly confident in. Overall, respondents with higher levels of perceived self-efficacy had lower levels of perceived danger, even if they did not necessarily have the knowledge to support their confidence. Even in the absence of knowledge, respondents with higher levels of perceived self-efficacy tended to believe that the danger was lower. In contrast, respondents with lower levels of perceived self-efficacy believed that not only were they more susceptible to threats because they would be unable to stop malicious users from doing anything, but they were more likely to consider the consequences to be severe.

Respondents who relied heavily on other people to take care of their security for them tended to believe that the danger of threats was lower. This was often because they felt that they were less susceptible to threats because someone else was ensuring that they did not have to deal with them and because they felt that the consequences would not be severe since that person could also remedy the consequences:

I feel like I have someone who's doing that job for me. If I didn't have that person I'd probably be a lot more... I'd probably be quite different. Like I would either walk around worrying all the time because I'm too lazy to do it or I'd do something about it. (Int-13)

Although this did not always mean that the respondent would not take steps to protect themselves if necessary, generally respondents felt that it would be unlikely that someone else would not be responsible for their security. As a result, respondents who exhibited higher levels of reliance believed that the danger of threats was lower because they trusted people to keep them safe.

Reduced levels of trust often served to increase perceptions about respondents' susceptibility to threats. Distrust has been linked to perceived vulnerability to crime (see Eitl \& Taylor, 2008). Although not directly linked to cybersecurity, cybersecurity breaches were described by some respondents as being akin to a violation of their sense of security. Respondents who were distrustful of people and sites online tended to avoid putting sensitive information on their computers or entering sensitive information on websites because they believed that they were highly susceptible to that information being misused: 
I use [the internet] to find information about buying things,

but I don't necessarily buy them. None of my account numbers are ever on it, I just don't trust it. (Par-2E)

Respondents with lower levels of trust made similar statements about not entering sensitive information online. Respondents with higher levels of trust tended to believe that people online were generally trustworthy and as a result would not take their information, indicating that their trust reduced their perception of susceptibility. Furthermore, respondents with higher levels of trust often believed that the consequences of breaches would be less severe, typically describing them as "annoying" rather than elaborating on more specific consequences.

\subsubsection{Summary of Relationships of Internal Factors}

Knowledge and perceived self-efficacy had the greatest effect on the other internal factors. These two factors had associations with almost all of the internal factors identified. Perceived self-efficacy often allowed respondents to feel confident enough in their ability to understand information about security to seek it out and increase their knowledge, which in turn further increased their confidence.

Higher levels of prior experience, knowledge, and security orientation were the most positively associated with awareness. Prior experience typically was the catalyst event that made respondents more aware of the symptoms of a breach. Knowledge allowed respondents to better judge whether the consequences they had experienced were the result of a cybersecurity breach. Overall, a general level of concern also allowed respondents to be more aware of threats because they were already concerned about them. In contrast, higher levels of reliance and trust tended to be negatively associated with awareness. Reliance allowed respondents to assign the responsibility for being aware of threats to someone else. Trust allowed them to believe that there would be few consequences that would be severe or that the incidents were so unlikely to happen that they did not need to be aware of the possibility.

Higher levels of security orientation and knowledge were positively associated with increased perceptions of severity and susceptibility. High levels of security orientation meant that respondents were already concerned about security. High levels of knowledge often meant that the respondents were aware of what the possible consequences could be and how threats were transmitted, typically resulting in greater concern over the possibility of a breach. Prior experience was usually positively associated with perceived susceptibility since respondents who had experienced past breaches felt it was more likely it would 
happen again. However, depending on the type of consequences experienced and their severity, prior experience could be either positively or negatively associated with perceived severity. Higher levels of perceived self-efficacy were often associated with lower levels of perceived severity and perceived susceptibility because these respondents felt that they could not only prevent breaches, but also remedy the consequences. High levels of reliance meant that respondents were less concerned about their susceptibility to threats because someone else would protect them, and less concerned about the severity of threats because they felt someone else would remedy the consequences. Finally, high levels of trust meant that respondents felt that the consequences of a breach would not be severe, and that they were unlikely to suffer from a breach because people and sites were generally trustworthy enough that they did not need to be concerned.

\subsubsection{External Factors}

External factors are factors that are present in respondents' external environments which relate to their security behaviours. External factors are comprised of symptoms, security messages, and computer use context. External factors influence respondents' awareness of threats, often by prompting reactions. They also influence certain internal factors of respondents, often serving to strengthen the effect of the internal factor. Finally, external factors influence respondents' perceptions of danger, typically by either providing the evidence that a breach has taken place or by providing an extra reasons for the respondent to be careful online.

\subsubsection{External Factors and Awareness}

Of the external factors identified during the data analysis, the two that had the greatest positive effect on awareness were symptoms of breaches and security messages (see Figure 5-6, p. 171). Symptoms of breaches often provided a catalyst event that triggered an increase of concern due to an increased awareness of a security threat. Johnston \& Warkentin (2010) indicated that without past experiences with cybersecurity breaches, individuals were likely to persist in believing that they were invincible to threats (p. 561). This was exemplified in the current research when several interviewees described how when they noticed that something was "wrong" on their computer it started a process where they would actively seek to increase their awareness in order to determine not only what had gone wrong, but in many cases, how to fix it: 


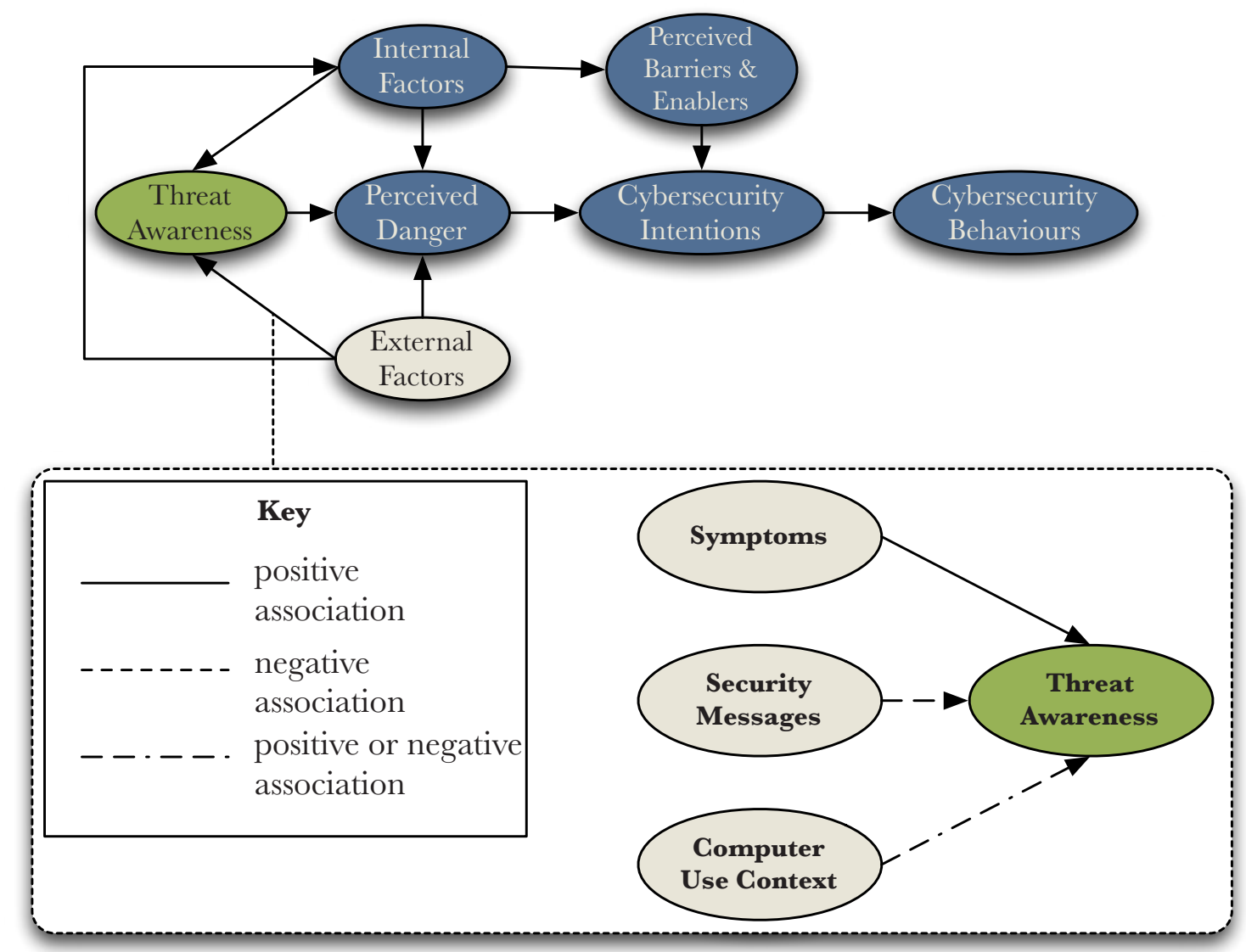

Figure 5-6. Influence of components of external factors on awareness

I figure if I have something going wrong, someone else has probably experienced it too. So, I generally trust that the information on those sites will at least get me started in finding something. So the people are knowledgeable and that's what makes me trust the information there. (Int-7)

As a result, the symptoms of threats were the catalyst event that led to more active security behaviours by the respondents. However, because there was no guarantee that a security breach had tangible symptoms, respondents who had not experienced such symptoms had their awareness influenced by other factors. Without awareness and knowledge of threats, respondents often indicated that things like slow computers and popups were indicative of breaches, but were not severe enough of consequences to warrant an increased level of awareness.

General security messages such as those from the news, websites, or trusted family members had the ability to increase respondents' awareness. However, in past research the result of media messages have been mixed (for example, see Thomson \& von Solms, 1998; Harrington, et al., 2006). Respondents in the current study talked about how they considered themselves to be more aware of threats because of the information they were given from other sources: 
- My husband would email me if he knew of a virus because they're on automatic. They go in and they're automatically sent things to say "a big virus is on it's way, make sure you've got this thing installed to protect yourself". (Int-5)

- Some of it is reading about it and hearing about it from reading about it on blogs and newspaper and stuff like that. (Int-13)

In both situations, the information that the respondents had gained helped to increase their awareness of threats. However, in the first case, the respondent was also more aware of safeguards because the security message had provided information about what steps she could take to protect herself. In the second situation, the respondent was more focused on increasing her awareness of threats rather than of safeguards. In this way, depending on the content of the message, the effect on awareness could vary. In addition, if respondents were not confident in their ability to act on these messages, they were less likely to credit security messages with increasing their awareness.

Unlike symptoms and security messages which could have positive effects on awareness, the effect of the computer use context was mixed depending on how much the respondents relied on others. When a respondent shared their computer with another person it often created a dynamic whereby one person was more responsible for the computer than the other(s). This could have the effect of increasing or decreasing awareness depending on how reliant the person was. One of the interviewees explained how sharing the computer with her husband helped make her more aware of threats, even though she did not necessarily know what steps she could take to avoid them:

[My husband] would spend a lot of time coming home saying; "oh my god, I've spent all day dealing with this virus issue at work". Which makes you more, raises your awareness. (Int-5)

However, this level of information sharing and increased awareness was not always present. Respondents who were very dependant on others to protect them had lower levels of awareness, essentially choosing to avoid security themselves. A participant who was less aware of threats described how her father took care of all the security in their household:

Dad is [responsible for our security], cause he's a photographer and our old computer had like all these photos on it... So like ever since, dad's been really good and backs everything up on this separate hard drive that he plugs in, like this metal box. (Par-1C) 
She later explained that she did not know what protective steps she could be taking and indicated that she would have to ask her father. However until that point she had seen little value in knowing what threats or safeguards to be aware of because she did not take responsibility for the security of the computer. These types of comments were particularly common among women whose husbands were responsible for their computers' security. As a result, awareness was lower when computers were shared and the individual did not take responsibility for their own security. However, if the respondent took greater responsibility for their security, computer sharing resulted in increased awareness. Respondents who shared their computer but felt that at least one other person was relying on them to keep the computer safe tended to have moderate to high levels of threat awareness. This indicated that the computer sharing context could trigger an increased sense of reliance which often resulted in a more negative outcome where it allowed individuals to feel that they no longer needed to be aware of threats since someone else was responsible for taking care of the problem.

\subsubsection{External Factors and Internal Factors}

Factors in the individual's external environment also appeared to influence certain internal factors (see Figure 5-7, p. 174). Symptoms of security breaches were a direct antecedent to prior experience. Although not all security breaches show tangible symptoms, respondents who experienced tangible symptoms of breaches were more likely to consider themselves to have had past experiences. Similarly, if a breach did not show symptoms immediately, it was often not until the respondent experienced some sort of trigger event, such as noticing the consequences of a breach or that their antivirus software had prevented a breach that they considered themselves to have experienced a breach.

The presence of symptoms was also associated with trust. Respondents who experienced the symptoms of security breaches tended to have lower levels of trust in people and websites. This appeared to be because they had a tangible sign that something had gone wrong. However, the effect of symptoms on levels of trust depended on the perception of the severity of the symptoms. If the symptoms were considered to be more "annoying" and less severe, they tended to have a minimal effect on respondents' trust. In contrast, where the symptoms were much more severe, such as "preventing access" respondents' trust was affected in a much greater way, and tended to decrease overall. Symptoms also influenced security orientation. Symptoms typically made people more concerned about their security. However, whether this initial concern translated into an ongoing concern depended on additional factors such as how the respondent viewed the severity of the consequences and the intentions of malicious users. 


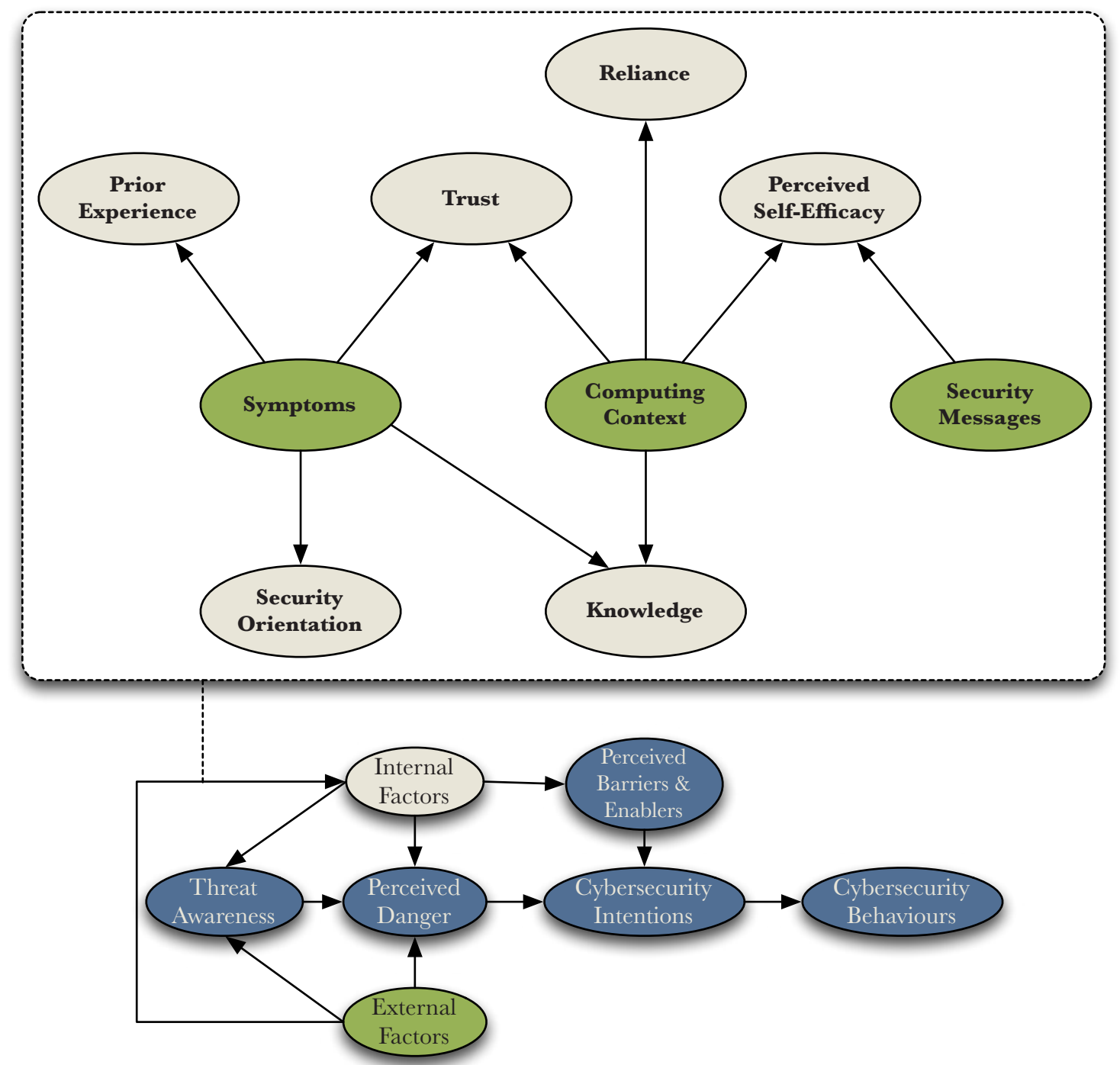

Figure 5-7. Influence of components of external factors on internal factors relating to home users' intention to engage in internet security behaviours

Respondents who shared their computers often demonstrated a level of trust in the other users of the computer because of the expectation that the person would not engage in any activities that could put the respondent in harm's way when using the computer themselves. In many ways, respondents felt that the fact that they shared a computer meant that they needed to be more trusting of other people who used the computer in order to avoid feeling like "a cop" about security. Although this did appear to have a positive influence on respondents' trust, it was more of a localised trust of people in the household rather than of potentially malicious users and sites. Computer sharing also tended to be associated with reliance. Respondents who shared their computers often either relied on someone else to protect them from threats or they felt that other users of the computer relied on them for ensuring that the computer was safe to use, for example: 
We share the same computer. It's actually my partner's, my husband's computer, but... I rely on [my husband] to be our technician at home (Par-1B)

While not all respondents who were reliant on others shared their computers, most did. Similar to how symptoms were an antecedent to prior experience, computer sharing tended to be present when respondents had high levels of reliance. Respondents with low levels of reliance did not have their levels of reliance affected as much by computer sharing as they were more often the ones who were relied upon by others who shared the computers. The effect of computer sharing on reliance was also reflected in how it affected respondents' perceived self-efficacy. Respondents who were heavily reliant on the person(s) they shared their computer with did not feel that they needed to increase their skills because someone else was doing the job for them.

Security messages primarily influenced perceived self-efficacy. In particular, respondents who received security messages at work felt that they were better prepared to be able to counter threats at home. As discussed earlier, in some cases, this increased level of awareness that the security messages generated had the potential to also generate an increased perception of self-efficacy even if it did not appear to be justified. Because the focus was on perceived self-efficacy rather than actual skill, security messages were seen to have a positive effect on perceived self-efficacy.

\subsubsection{External Factors and Perceived Danger}

The relationships between external factors and perceived danger were generally mixed (see Figure 5-8, p. 176). The only external factor that had an overwhelmingly positive effect on perceived susceptibility was the effect of symptoms, while computer use context was the only factor that had an overwhelmingly positive influence on perceived severity. Symptoms of cybersecurity breaches were positively associated with perceived susceptibility because in almost every case, respondents considered themselves to be less susceptible to threats if they had not experienced the symptoms of a breach. In contrast, respondents who believed they had experienced breaches were more likely to describe symptoms they associated with a breach as the evidence that such a breach had taken place.

Although symptoms tended to have a positive influence on perceived susceptibility, their influence on perceived severity depended more on how the respondent viewed the consequences. For example, respondents who believed that getting multiple pop-up ads were a sign of a cybersecurity breach tended to consider the overall severity of threats to be lower, which in turn reduced their overall perception of danger. In contrast, respondents 


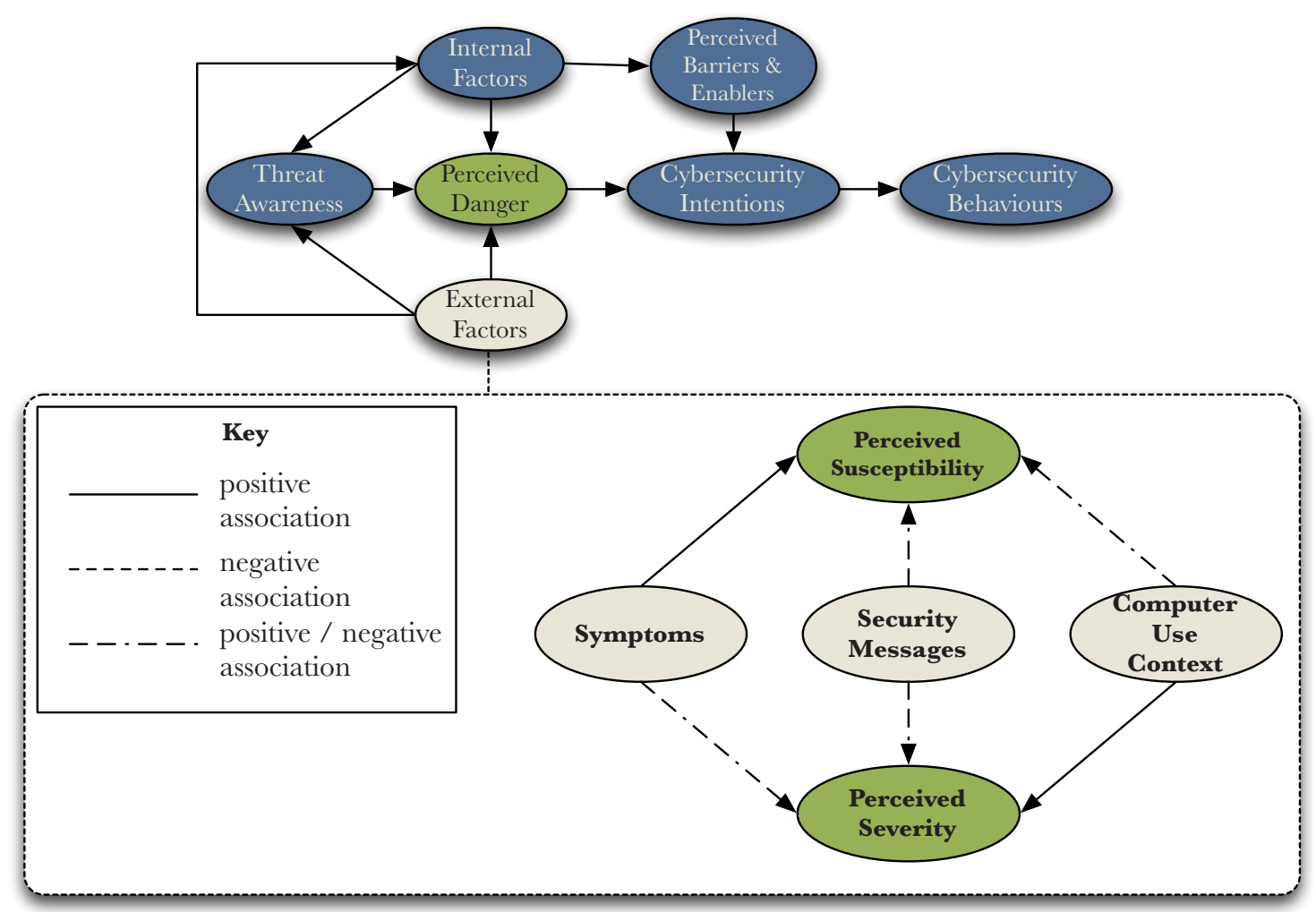

Figure 5-8. Influence of components of external factors on perceived susceptibility to and severity of cybersecurity threats

who lost access to computers, or had data corrupted to the point where they could no longer use it tended to interpret the severity of threats as being higher because the symptoms they had experienced in the past led them to associate cybersecurity breaches with more negative consequences.

While this shows how symptoms can influence both severity and susceptibility, the main danger with waiting for symptoms of a breach to appear is that it requires that (1) the threat have tangible symptoms, (2) the individual be able to recognise the symptoms, and (3) that the individual associates those symptoms with a cybersecurity breach. As a result, if these three requirements are not met in association with the symptoms, it may be too late to prevent the potential consequences, leading to a greater investment of time and money in order to remedy the breach.

Security messages had positive and negative influences on both perceived susceptibility and perceived severity. Security messages had the greatest positive effect when respondents had a clear sign that something was happening or had gone wrong and when they had the requisite level of knowledge to understand the security messages presented by their protective software. For example, some respondents reported how the security messages informed them about threats they were facing: 
There have been several attacks, you know, people trying to access the IP and stuff like that... I'm just concerned about others trying to steal my information. And also steal account details. And passwords. (Int-18)

In this case, the message increased her concern over her susceptibility to threats because she was experiencing the breaches. It also increased her concern over the severity of the consequences because she was concerned that malicious users were trying to access information that she considered to be sensitive, including documents, passwords, and account information. However, if the respondent did not consider the consequences to be severe, this often resulted in their downplaying the danger of the threat. For example:

Occasionally the virus scanner detects something, and it usually deals with it. So it's not that much of an issue. (Par1A)

In this case, although the respondent had experienced a breach, he did not associate it with increasing his future susceptibility to threats, nor did he regard the consequences as being particularly severe. As a result, the security message that indicated that the protective software had stopped the breach led him to feel that the danger of the threat was less. This illustrates how security messages can have both positive and negative effects on perceived danger depending on how the individual interprets the messages they receive.

When respondents shared a computer with at least one other person, this often influenced their perceptions of susceptibility to and severity of threats. However, the way in which the computer sharing affected their perceptions of severity and susceptibility depended on their role with the computer. For example, respondents who considered themselves to have the primary responsibility for protecting the computer were more likely to believe that they were put at greater risk (perceived susceptibility) by sharing their computer with a person who they considered to have lower levels of knowledge and concern about security:

I think I'm most concerned about my husband because he would download stuff and I'm really concerned about the software that he would be downloading, and what he thinks he's downloading. So that sort of thing freaks me out (Int-2)

Because of this, these respondents often felt they had to either police what the other person was doing or they had to take responsibility for ensuring the computer was secure before they used it. In contrast, respondents that shared a computer but felt that the person they shared the computer with had the primary responsibility for protecting the computer often felt a diminished sense of susceptibility to threats due to their high levels of reliance. 
Finally, sharing a computer with another person tended to create an increased perception of the severity of a breach. If a security breach were to occur as the result of a failure to engage in safe behaviours online, the consequences would not be limited to the person who was responsible for the breach.

A lot of the time I'm concerned that he uses my computer and does activities that he has not really, you know, put security as a priority, and because of this it might compromise my files. (Int-4)

Because of this, it appeared that respondents who shared computers were more likely to be concerned about the severity of a breach than if they were the only person using the computer.

\subsubsection{Summary of Relationships of External Factors}

Overall, external factors appeared to influence awareness, internal factors, and perceived danger. Symptoms and security messages had the greatest effect on awareness. Symptoms provided evidence of a breach while security messages provided an explanation of why an individual needed to be concerned about the breach. The combination of these sub-factors increased the perception that the information about the threat was relevant to respondents, which in turn increased their awareness of threats. In some cases, whether or not the computer was shared with another person also helped to increase awareness of threats. However, this depended on the way that the respondent reacted to what the other person was doing in regards to security.

Symptoms of breaches increased respondents' knowledge and their concern about security, reduced their trust in others, and were the catalyst for respondents to form opinions about the effects of breaches. Even where symptoms were not present, the lack of symptoms often had an equal but opposite effect on respondents, for example, the lack of symptoms was often responsible for a perception that there was no need to be concerned about security. Whether or not a person shared a computer influenced their trust in others, reliance on others to fix problems, knowledge of threats, and perceived self-efficacy. In many cases, the effect of computer sharing depended on whether respondents considered themselves to be responsible for the security of the shared computer or whether they simply used it. If they only used it, the effect was typically that they would not feel that it was as necessary to be as knowledgeable about threats or know as much about how to prevent them. The main benefit of security messages was that they tended to increase perceived self-efficacy because they often provided respondents with what they considered to be 
enough information about threats, even if they were not entirely certain of what steps needed to be taken.

Symptoms of security breaches typically increased the perceived susceptibility to threats because they provided tangible evidence that something had gone wrong. Computer sharing context had the potential to increase the perception that the consequences of the threats would be more severe if more than the respondent's data was damaged. The remaining effects of the external factors on perceived danger varied based on the situation. For example, whether or not symptoms had a positive influence on perceived severity depended on the individual and their beliefs about what malicious users' intentions were. Overall, external factors often helped to trigger certain responses.

\subsubsection{Perceived Barriers and Enablers}

Perceived barriers and enablers were additional factors that hindered or encouraged respondents' cybersecurity behaviours. As discussed in Chapter 4, the main barriers to engaging in cybersecurity behaviours were the perceived time and financial costs, as well as a lack of prompting about the need for security. The perceived enablers were often less clear. Respondents frequently indicated that the steps they took were done to prevent the consequences of breaches from occurring or to maintain a feeling of security. Perceived barriers and enablers were influenced by respondents' internal factors. Lower levels of internal factors such as knowledge and security orientation often contributed to barriers having a greater effect. Similarly, higher levels of these factors often minimised the effect of barriers and made it more likely that respondents would be able to identify reasons to take protective steps, even with the presence of the barriers. Perceived barriers and enablers also contributed to respondents' security intentions, in conjunction with perceived danger. Respondents with lower levels of perceived danger were more likely to be affected by barriers while respondents with higher levels of perceived danger were likely to believe that the benefits of engaging in security behaviours outweighed the barriers to doing so.

\subsubsection{Internal Factors and Perceived Barriers}

Although seven internal factors were identified as being relevant to influencing home users' cybersecurity intentions, three were of particular importance in examining how much the perceived barriers prevented respondents from intending to engage in safer behaviours online: knowledge, perceived self-efficacy, and security orientation. The 
presence of high levels of knowledge, perceived self-efficacy, and security orientation tended to mitigate the effect of the perceived barriers on respondents' intention to engage in protective behaviours. While both barriers and enablers influenced respondents' intention to engage in cybersecurity behaviours, enablers were typically influenced by a desire to protect either family members or the respondents' own personal sense of security. As a result, the barriers were more greatly influenced by respondents' internal factors. These relationships are explored in greater depth in this section.

Respondents with lower levels of knowledge believed that it was both costly and time consuming to engage in security behaviours. Both of these barriers were identified by Furnell, Bryant, and Phippen (2007) in their study on security perceptions of home users. Respondents in the current study indicated that they did not know of safeguards that were less costly or took less time and could protect their security. For example, one of the interviewees who indicated that cost was a particularly large barrier for her stated:

The cost is the most, [pause] the biggest barrier at the moment...If I know there are other ways to protect myself online, my data, of course I will go for it. (Int-12)

She further indicated that if she knew of additional safeguards or free safeguards, then she would use those. However, her lack of knowledge and awareness of these safeguards limited her ability to engage with them. As a result, she believed that most safeguards were very costly to use. Furthermore, this limited her use of such safeguards on other computers in the house. This was further compounded by a belief that free antivirus software was less effective than paid antivirus software (see section 4.7.1). In addition, respondents with lower levels of knowledge also felt that as long as a computer had "good" antivirus software, there was no need for any further steps to be taken. These respondents often believed that taking any additional steps to protect their cybersecurity would be too time consuming compared the to the amount of additional protection these steps would offer. In contrast, respondents with higher levels of knowledge were either (a) unconcerned by the cost of taking additional precautions or (b) were already aware of low cost or free alternatives for protecting themselves. As a result, these respondents were not as concerned about the cost of additional forms of protection.

Safeguards were also considered to be very time consuming when respondents had lower levels of knowledge:

Time, time, time... you want it, you want it now. You know, it's really infuriating if it's holding up your life. (Int-14)

And often the safeguards that were considered to be the most time consuming were the ones that respondents knew of, but did not use. Respondents who were more knowledgeable 
often considered the time that it took to protect their computers to be less than respondents with lower levels of knowledge. Respondents with higher levels of knowledge were also more likely to consider the time it would take to protect their computers to be less of an issue because they understood the benefits of taking the protective steps. However, even amongst respondents who were knowledgeable, there was a limit to how much time a safeguard could take before they decided that the time commitment was too high. This was usually when the time it would take to safeguard the computer would be more than it would take to recover from a breach.

Respondents with higher levels of perceived self-efficacy tended to be less concerned about the cost of safeguards because they were often able to identify free, often opensource versions of protective software that could be used. Respondents with lower levels of perceived self-efficacy were often less aware of free alternatives, primarily because they believed that the paid versions would be more effective, making them better able to supplement the skills that the respondents considered themselves to be lacking.

Individuals with high levels of concern about their security tended to be affected the least by barriers because they understood the benefits of engaging in protective behaviours. Although respondents with higher levels of security orientation were aware of the barriers, they were less affected by them overall. One of the respondents who had higher levels of security orientation explained why he felt that the barriers did not inhibit his security behaviours:

People say you can take a lot of actions to keep yourself healthy, but the sort, all the activities you perform, they can diminish the time you have to do something else. So the opportunity cost of the backup is [high]... The opportunity cost of not doing that? (Int-11)

In this case, he considered the cost of not performing security behaviours to be much higher than the time cost of engaging in these behaviours. This sort of view was common amongst people who were very concerned about their security since they were much more concerned about how much time it would take to recover. As a result, their security orientation helped diminish the effect of the barriers on their security behaviours.

Overall, internal factors tended to be negatively associated with perceived barriers. As respondents' knowledge, perceived self-efficacy, and security orientation increased, the effect of the barriers often lessened. However, it is important to note that in many cases, these internal factors did not work alone to mitigate the effect of the barriers. Often at least two of the three main internal factors needed to be moderate to high before respondents started to consider the barriers to be less important. As a result, there were respondents who had high levels of perceived self-efficacy, but low to moderate levels of concern and 
knowledge, and were more affected by barriers than their counterparts with higher levels of perceived self-efficacy and moderate to high levels of knowledge and/or concern.

\subsubsection{Summary of Relationships of Perceived Barriers and Enablers}

Overall, the barriers to engaging in cybersecurity behaviours were much stronger than the enablers. While having strong feelings about the benefits of security could help respondents with more moderate perceptions of danger to overcome possible reluctance, it was rare that respondents felt so strongly about the benefits of security. Barriers had the strongest role when respondents already felt that the danger of threats was moderate to low. With lower levels of perceived danger, respondents were reluctant to take on security behaviours where they perceived the time or financial costs to be high. As a result, external barriers were another way for respondents to justify why they did not need to take steps to protect themselves, since they believed there was little reason to spend large amounts of money or time protecting themselves when the danger was so low.

\subsubsection{Security Intentions}

The overall perceptions of danger combined with the perceived barriers and enablers led to the formation of respondents' intentions to engage in protective behaviours online. In order for respondents to have the strongest intention to engage in such behaviours, they typically had a high level of perceived danger and were minimally affected by barriers to engaging in cybersecurity behaviours. The result was that respondent's cybersecurity intentions influenced their behaviours.

\subsubsection{Perceived Danger and Security Intentions}

The greater the perception of danger regarding online threats, the more likely respondents were to engage in protective behaviours. Without a sufficient perception of danger, individuals are more likely to consider the danger to be irrelevant (for example, see Blanton, et al., 2001; Furnell, Bryant, and Phippen, 2007; Johnston \& Warkentin, 2010). Overall, respondents needed to feel that not only was the threat likely to occur, but also that the consequences of a breach would be severe enough for them to want to change their behaviours. As discussed earlier, while severity and susceptibility do not appear to influence 
each other, they do work together to form a combined perception about the danger of the situation. The greater the perceived danger, the more likely that the respondent would be to intend to engage in protective behaviours. One of the interviewees who felt there was more she could be doing to protect herself commented:

In terms of what I am doing now, I'm extremely conscious of what can happen. So I think it has an impact because if I had like, a huge amount of self-value, then I would actively seek out to be more protected. (Int-18)

Like many respondents, this particular interviewee associated the severity of the consequences with the perceived value of her data. In this case, the disconnect between her perception of the likelihood of having something happen and the severity of the consequences, she felt she was not doing enough to protect herself. Furthermore, these perceptions influenced her intention to protect her computer. When there was a difference between perceived susceptibility and perceived severity, the intention to engage in cybersecurity behaviours tended to be more moderate. Respondents with similar disconnects tended to engage in basic protective behaviours, but did not feel enough concern over the danger of threats to take steps that they considered required more effort, were more time consuming, or cost more money.

\subsubsection{Perceived Barriers \& Enablers and Security Intentions}

Perceived barriers were particularly influential with regard to respondents' security intentions when there was a disconnect between the perceived susceptibility to, and severity of, a threat resulting in a moderate perception of danger. Where the perception of danger was more moderate, the effect of the perceived barriers and enablers tended to be the factor that either pushed respondents to take further action or to stay with whatever steps they were already taking. In many cases, it came down to a perceived trade-off between convenience and determining what level of risk was acceptable to the individual. As one of the interviewees described:

The backup drive I have is connected to my computer. If my computer is compromised, people are able to get access to the drives connected to my computer, including my backup... I decided if I'm not doing high risk stuff, I don't need to be actively backing up my stuff. (Int-4)

Although this particular interviewee indicated that he was well aware of the benefits of 
using a backup in order to safeguard his data he decided that the time costs were too high compared to the amount of risk he was willing to take based on his assessment of the danger of the threat. Therefore, although in this case the perceived severity of the consequences was higher, the perceived likelihood was low enough to result in a more moderate level of perceived danger. As a result, the barriers became more prominent and affected his intention to engage in certain protective behaviours.

\subsubsection{Cybersecurity Intentions and Cybersecurity Behaviours}

In some cases, the biggest danger to people was themselves. Furnell, Bryant, and Phippen (2007) found that $8 \%$ of their respondents indicated that there was nothing that stopped them from protecting their security, the just did not do it (p. 416). Despite statements by the current study's respondents that they knew what they should be doing to protect themselves, they still did not take these steps. One participant's comments summarised what many of the respondents said when discussing the steps they took to protect themselves:

I know what I should do, but I don't do it. So I'm my own worst enemy in that I know what I should do [but I don't do it]. (Par-2D)

Approximately $85 \%$ of respondents indicated that they knew there were additional steps, beyond the basic protective steps they already took, that could be taken to protect themselves but that they did not do for various reasons. The main reasons were either because they did not perceive the danger to be high enough or they considered the barriers to changing their behaviours to be too high.

Although there was the risk of people knowing better but not following through, in other cases, respondents' security intentions did guide their behaviours. This was generally found where respondents did not know of any additional behaviours that they could undertake to protect their cybersecurity. For example, an interviewee described why she considered herself to be safe:

... at the moment the way I'm doing it, like using all the sources, emails, backups and everything. I think, I can, I can consider myself as a safe user. (Int-1)

She subsequently explained that there were no other steps she thought she should be taking, exemplifying a respondent whose intentions closely matched the behaviours. As indicated in Chapter 4, 11 of the 20 interviewees indicated they were doing all the steps they intended 
to do, while the remaining nine interviewees had a disconnect between what they knew they should do and what they actually did. This disconnect appeared to be indicative of a lower intention to engaging in cybersecurity behaviours, usually because of a lack of perceived danger or too high of perceived barriers.

\subsubsection{Summary of Security Intentions}

The greater the perceived danger of the threat, the more likely respondents were to engage in protective behaviours. Often the greatest barriers to behavioural security intentions were not the external barriers such as cost and time, but rather a lack of perceived danger. A lack of perceived danger was often the result of internal factors combining in a way that meant that respondents did not feel that it was likely that they would be victims of breaches and even if they were, that the consequences of these breaches would be minor annoyances. If the perceived danger was low to moderate, the effects of external barriers became more prevalent. For example, if a person was only somewhat concerned about the danger of threats, if they considered the cost of safeguards to be too high or to require too much time, they were less likely to engage in safer behaviours. However, respondents who were very concerned about the danger of threats were much more likely to dismiss the external barriers as being unimportant. Therefore, both the perceived danger and perceived barriers need to be considered in relation to intention to engage in safer practices.

\subsection{Chapter Summary}

In this chapter, the conceptual model from Chapter 3 was revised based on the data collected in this study. Based on the findings, without awareness of threat, respondents were much less likely to consider the danger of threats to be high. If the danger of threats was considered to be low, respondents were much less likely to have strong intentions to engage in security behaviours. Along with awareness, perceived danger was influenced by respondents' internal factors and external factors. Knowledge and perceived selfefficacy had the greatest effect on other internal factors as well as on perceived danger and awareness. In the next chapter, the relationships from the model and the most influential of these factors are elaborated on further through the identification of user types that share similar features and behaviours. 
Braun 


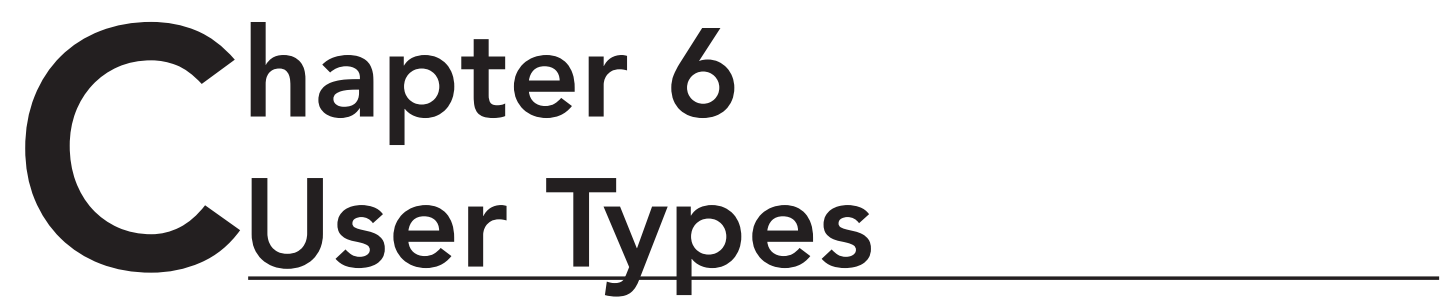

\subsection{Introduction}

Chapter 4 presented the findings from the data analysis. Based on the findings, a revised model was developed which was explained and justified in Chapter 5. The revised model presented an overall view of how different factors interacted in order to influence home users' security behaviours. However, in that model it became clear that the internal factors were particularly important to influencing respondents' awareness of threats and intention to engage in safer behaviours. Further examination showed how particular combinations of factors were more likely to lead to certain cybersecurity behaviours. From these combinations of factors, five main types of individuals are identified. The identification of these user types answers research question 2 which asked how the factors related to home users' cybersecurity behaviours influenced their practices. The user types that are presented in this chapter also help to meet research objective 3 which is to determine how awareness of threats and safeguards influence home users' cybersecurity behaviours.

In this chapter, the development of the user types is explained. This is followed by an exploration of the average home computer user as described by the respondents. The profile of the average user is presented as a starting point for looking at respondents' beliefs about computer security and as a starting point against which to compare the identified 
respondent types. This is followed by an exploration of each of the user types, their key identifying characteristics, and an overview of the commonly identified behaviours. Finally, the identified user types are compared based on similarities related to perceived self-efficacy, knowledge, and behaviours.

\subsection{Creating Security User Types}

From the identification of the internal factors in Chapters 4 and 5, it was possible to see how respondents' internal factors influenced their awareness of threats, their perceived danger of threats, and the perceived barriers to and enablers of engaging in security behaviours. The user types explored in this chapter further illustrate the interactions between the internal factors and how the identification of these users can be used to address security issues. Throughout the data analysis, certain factors appeared to be key in identifying how individuals would not only perceive the danger of online threats, but also in identifying the behaviours they were most likely to undertake.

In section 5.4.3, the interactions between the internal factors showed that knowledge, prior experience, reliance, security orientation, perceived self-efficacy, and trust were particularly important in influencing respondents' behaviours. The final internal factor of ego-protection is not included in the user types. As will be discussed in Chapter 7, laziness was often used to indicate a lack of concern about security. In addition, both laziness and boastfulness were behavioural manifestations of combinations of internal factors such as knowledge and perceived self-efficacy. Therefore, ego protective behaviours are not considered in this section. Respondents' awareness of threats and of safeguards were also particularly important when they were considered in relation to their demonstrated awareness. Although these eight factors were identified as being particularly important in this study, there may be additional factors which are influential in determining home users' cybersecurity behaviours. The value of exploring these user types is to provide a lens through which users' security behaviours can be examined and explained.

To provide a visual representation of the identified factors, each was assigned a numerical value from 1 (low) to 5 (high) based on the specific answers or the interpretation of respondents' answers to questions. By assigning a numerical value to respondents' answers to relevant questions in relation to each of the factors identified, it was possible to generate radar charts for each of the user types in order to help illustrate the relevance of each of the factors for each user type (see Figure 6-1, p. 189). For an overview of how these rankings were assigned to each of the factors, see Appendix 6.

Based on the data analysis eight factors were identified as being particularly relevant in identifying similar types of respondents. In Figure 6-1 (p. 189), knowledge was placed 
opposite perceived selfefficacy in order to help display discrepancies between how capable respondents thought they were in terms of protecting themselves online in comparison to how knowledgeable they actually were. Prior experience was placed opposite security orientation as in many cases these two factors were closely linked, with respondents who had high levels of concern typically having experienced breaches in the past. Threat awareness and safeguard awareness were placed opposite each other as the combination of the two creates an overview of what would be included in cybersecurity awareness. Finally, reliance was placed opposite trust because of the need to demonstrate trust when relying on a third party's knowledge and skill. Before exploring the user types in more depth, it is useful to first explore what the respondents felt the average user was like. This provides a starting point from which it is possible to look at whether the respondents' perception of the average user was accurate and whether it fit any of the identified types of respondents.

\subsection{Portrait of a "Normal" User}

To put each of the user types into context, it is possible to look at what respondents considered to be the "normal user." The "normal user" is not a separate user type, but rather provides an overview of the supposed person that respondents compared themselves to when they indicated that they felt they were above or below average in some way. This is distinct from the "average respondent" that is discussed in section 6.5.1 which focuses on the mathematically average respondent based on the data analysis of the individuals involved using the rankings set out later in this chapter.

The features of the normal user were based on respondents' comments specifically in response to questions about average users and on comments that included information about average users indirectly. The questions asked included questions about the average 


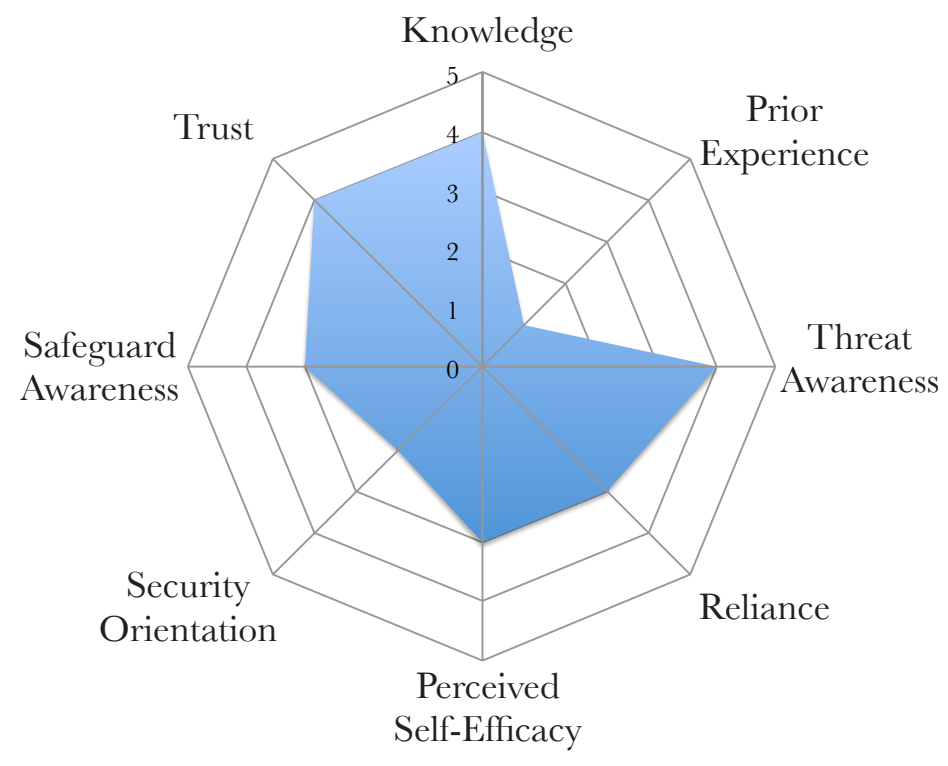

Figure 6-2. Overview of the key features of the perceived "normal user" as described by respondents users' levels of awareness and knowledge as well as the steps that the average user would take to protect themselves online. Through the course of discussions, further responses included comments about normal users' levels of trust, selfefficacy, concern about security, past experience, and reliance (see section Figure 6-12). The most commonly provided descriptions of normal users by respondents yielded the description of what was considered to be the normal user.

The perceived normal user was considered to have moderate to high levels of both knowledgeable and threat awareness. They were moderately aware of the safeguards they could use, but were not necessarily confident in their ability to protect themselves online. They tended to have a moderate level of reliance on others, but also tended to have moderate to high levels of trust that people would not harm them via their computer. However, there was also a belief that the average user was also generally unconcerned about security breaches and had not experienced a breach before.

Respondents commonly assumed that the normal user was knowledgeable about threats because cybersecurity behaviours and knowledge were a part of everyone's daily lives. As one of the participants explained:

Several years ago [the normal user] probably wouldn't have been as aware [of threats]. But now, it's like really ingrained in the culture really. You know what viruses are, and you know not to click on the email from the guy in Africa who is a prince and is going to give you $\$ 5,000$ if you just give him your bank account number. (Par-1C)

Comments such as the one above suggest that not only are people aware of the most common threats, but also that they are aware of the basic safeguards they need to take to 
protect themselves. However, the general feeling amongst respondents was that the normal user was more aware of the threats than of the safeguards. As a result, threat awareness was considered to be moderate to high (4) while safeguard awareness was moderate (3). In many cases respondents' also seemed to assume that awareness of threats was equivalent to understanding what the threats were. The result of this was respondents assumed that normal users' knowledge was similar to their awareness, moderate to high (4).

Despite the perceived awareness of threats, respondents overall believed that most people were not particularly concerned about cybersecurity threats and breaches. For example:

I think most people are at least moderately aware of these threats. But [they] are less concerned in terms of taking action to protect themselves from these, from some of these threats. (Par-1A)

Statements about low levels of concern were often used by respondents to justify why they felt that the average user would not take many steps to protect themselves online. As one of the participants summarised:

I think people are aware. But I don't think they do anything about it. It's like, yeah, I know there's problems, but do I change my behaviour because of it? No. (Par-2D)

Comments like this showed how most respondents considered the normal user to be aware of the threats, but with little concern about their cybersecurity which typically meant that the normal user took minimal protective steps. As a result, the normal user's security orientation was considered to be low to moderate (2).

Comments about the likelihood of a person having experienced a previous cybersecurity breach suggested there was a general belief that it was unlikely to happen in the future because it had not happened yet. Furthermore, there was a belief that most people had not experienced cybersecurity breaches. Therefore, prior experience was low (1). These beliefs about a lack of experience with breaches further contributed to a general perception the people and sites were typically trustworthy, which meant that people were more likely to do things online that could be risky. For example, because normal users were considered to be trusting, they were also believed to engage in risky behaviours. As two of the focus group participants discussed: 
The average person opens up way too many random emails.

(Par-2A)

And they go to too many sites too. (Par-2E)

Overall, the normal user was considered to have moderate to high levels of trust (4) in people and websites. If something did go wrong there was a belief that banks or someone else would remedy the issue. This suggested that although people expected the normal user to be competent enough to know how to protect themselves, they did not necessarily do so, which meant that someone would have to fix the problem. This indicated moderate levels of both reliance (3) and perceived self-efficacy (3).

Overall, this presents a picture of a user who is knowledgeable about the threats and aware that they exist, but who does not necessarily take the steps they know they should. This failure to act often appeared to be blamed on a lack of prior experience with threats, a high degree of trust in people and websites, and a low concern about security overall. This normal user provides us with a starting point from which to look at the types of users identified in this study. Later, in section 6.9, the normal user is compared to the other security types as well as the average respondent.

\subsection{User Types}

The combination of the factors identified in section 6.2 led to the creation of five main user types, each associated with a specific animal that exemplified the traits of the type. Ostriches typically ignored security risks in the hope they would simply go away. Mice were typically more aware of threats than their Ostrich counterparts, but were often still uncertain which resulted in their being timid in their security behaviours. Cockerels typically wanted to show off their knowledge, at times even overstating their knowledge to appear more secure to others. Coyotes tended to consider themselves as particularly clever, and often took security risks if they deemed the rewards to be worthwhile. Finally, Dark Horses tended to conceal their knowledge, often appearing to be Mice until their actual knowledge was revealed. Each of these types is explained in greater detail in the subsequent sections.

\subsubsection{Ostriches}

Much like the myth that the ostrich buries its head in the sand when attacked, hoping that the attacker will go away, Ostriches tended to bury their heads in the metaphorical sand 
in the hope that cybersecurity threats would cease to exist if they ignored them. Ostriches often tried to avoid dealing with threats and to convince themselves that there was no need to worry about them. This was often because they were either ignorant of the threat and did not want to learn about what threats could exist or because they were able to justify why they did not need to be concerned about them.

\section{FEATURES}

Ostriches were typically characterised by their moderate to high levels (4) of reliance on others, combined with lower levels (1) of perceived self-efficacy, threat and safeguard awareness, knowledge, and prior experience (see Figure 6-3). This combination of features allowed Ostriches to convince themselves that many threats online were not serious enough to worry about. In some cases, they believed that someone else would prevent or remedy any security issues. In other cases, Ostriches were able to justify to themselves why they would not be targeted by a malicious user. Three of the respondents in this study were most closely associated with the Ostrich user type.

In many cases, these beliefs were closely linked to Ostriches' perceptions about the intentions and motivations of the users who create malware and engage in malicious hacking. Often Ostriches tried to ignore the threat posed by malicious users by essentially convincing themselves that the threat was nonexistent. Ostriches frequently did this by convincing themselves that malicious users' intentions were benign rather than malicious (for example, the belief that malicious users were simply trying to show how smart they were, or the belief that the result of a security breach was that the person was inconvenienced but did not suffer any real harm). Ostriches tended to believe that malicious users were not truly malicious. This was because Ostriches were often trusting of people and tended to believe that it was unlikely that a person or website would cause them harm.

Ostriches' lower levels of knowledge also appeared to contribute to their trusting nature and their beliefs about malicious users. Respondents with lower levels of knowledge tended 
to believe that malicious users' intentions were benign because they did not understand what they could possibly have on their computers that someone else would be interested in. Ostriches' beliefs about malicious users appeared to be more influenced by the media's portrayal of "hackers" which has tended to glorify them as people trying to show off how smart or talented they are (for example, see Skibell, 2002) or to engage in hacktivist activities. ${ }^{1}$ While hacktivism made a rise in 2011, the primary motivation of malicious users has been financial (AFP, 2012). The belief that malicious users' activities are only politically motivated or simply a way to show off appears to cause Ostriches to believe that they will not be targeted since they do not have anything they consider to be of value. However, home computers have often been commandeered by both hacktivists and other malicious users to carry out attacks against businesses and governments via botnets and other malicious techniques (see Adhikari, 2012). Therefore, although Ostriches often seemed unconcerned about threats because they did not suffer any direct harm, they may have been unknowingly contributing to attacks against others, or have been affected as a result of an organisational data breach.

Ostriches' lower levels of understanding of both threats and malicious users motivations contributed to their lower levels of threat (1) and safeguard (2) awareness. In particular there seemed to be a lack of awareness of who was likely to be targeted by malicious users. Ostriches often believed that only companies and governments needed to be concerned with cybersecurity breaches. With lower awareness of threats there tended to be a somewhat increased level of dependence on the media for information about threats. However, the news media has tended to favour corporate cybersecurity threats over threats to home users. In a search of Google News for stories between 23 February - 23 March 2012, there were approximately twice as many stories about malware in organisations than there were for malware at home. ${ }^{2}$ The media has favoured stories about organisational threats consistently in recent years. This trend is especially important to consider if Ostriches are dependant on traditional media, such as newspapers, magazines, and news reports, to provide them with information about cybersecurity threats. If people are primarily given information relevant to businesses it is understandable why many people would assume that home users do not need to be concerned about threats. A lack of information relevant to home users can then cause them to be more likely to try to ignore the threats because they do not see them as relevant (Johnston \& Warkentin, 2010). If users do not see threats as being relevant

$1 \quad$ Hacktivists are "hackers who break into systems for political reasons rather than monetary ones" (Adhikari, 2012). While hacktivists can be malicious or non-malicious, Ostriches tend to assume that the activities are non-malicious. A prominent example of a hacktivist group is Anonymous.

2 I conducted a search on news.google.com on 24 March 2012 for stories dated between 23 February 2012 and 23 March 2012. The first search was done using the keywords "malware" and "home" which resulted in 733 results. A second search was done using the keywords "malware" and "business" with a result of 1310 results. Google News was used in order to obtain aggregated results from many sources rather than basing the result on a single news source. 
to them, they seem to have a greater need for a catalyst event that triggers them to engage in better online security.

Often Ostriches had not previously experienced cybersecurity threats or breaches, which resulted in low levels of prior experience (1). While this raises a question of whether they were unaware of a past breach due to their lower levels of cybersecurity knowledge, what was more important was that they did not believe a breach had happened. This lack of prior experience with cybersecurity threats and breaches often meant that users did not see the value in taking additional steps because they did not have the necessary context to make online threats relevant to them. This often resulted in respondents expressing feelings of invulnerability. They often believed that if a breach had not happened yet, it probably was not going to.

Despite the fact that Ostriches often dismiss threats as being irrelevant or with few consequences, one reason for this appears to be due to an underlying fear of what could happen if a cybersecurity breach did occur. For example, a respondent who demonstrated many Ostrich characteristics stated:

When there's a virus out there I won't touch the computer. I won't touch it those days that they say it's going around because if it happens I don't know what I'm going to do. (Par-3D)

Ostriches often noted that they would not know what to do if something happened. Bandura (1977) stated that often "fears and deficits [of coping skills] are interdependent. Avoidance of stressful activities impedes development of coping skills, and the resulting lack of competency provides a realistic basis for fear" (p. 199). As a result, Ostriches could become stuck in a cycle whereby they are too afraid of what could happen to do anything to protect themselves online and as a result their fear is increased because they do not have the skills to protect themselves. This may have further contributed to their ignoring of threats since researchers have suggested that excessive levels of fear can cause people to ignore threats (Johnston \& Warkentin, 2010; Ruiter et al., 2001), which in turn returns back to Ostriches being less knowledgeable, skilled, and confident because they ignore the threat. This can then have the further result of decreasing users' awareness of both threats and safeguards because they do not see the need to be aware of either. 


\section{BeHAVIOURS}

Ostriches' security behaviours often focused on ignoring the presence of threats in order to avoid facing them. Ostriches took the fewest steps to protect themselves of all types of users, and as a result tended to rely heavily on antivirus software or other people to protect them from threats. One reason Ostriches appeared to be so dependent on other people to protect their security is because of their low levels of knowledge and perceived self-efficacy. Often times Ostriches were able to ignore threats because they were not knowledgeable enough to understand why these were threats or what the potential consequences could be. If an Ostrich did acknowledge the threat, lower levels of perceived self-efficacy meant that they would seek out help, but this would only happen if they could no longer ignore an issue that they did not have the skills to address.

One problem that at times resulted from Ostriches ignoring threats is that they engaged in some risky behaviours. However, Ostriches, did not knowingly engage in risky behaviours. Instead, they either did so because they were naïe of the risk or because they had convinced themselves that the behaviour they were engaging in was not actually risky. For example, Ostriches were more likely to illegally download movies and music from websites, even though these downloads are often "poisoned" by malicious users (see Chmielewski, 2012). Thus, Ostriches can unknowingly engage in risky behaviours, and as a result be unaware of the need to protect themselves online.

Compared to other users, Ostriches often took fewer steps to protect themselves because they did not feel the need to do so. This was because they had lower perceptions about the danger of the threat overall. The lower perception of danger was the result of lower perceptions of susceptibility (e.g. individuals will not be targeted as much as organisations), lower perceptions of severity (e.g. "I have nothing of value on my computer"), and strong beliefs in the effectiveness of the safeguards they use.

Ostriches generally avoided thinking about online threats as much as possible. When they could not avoid threats, they tended to state that the consequences of the threats would not be as severe as reality suggested. A key example of this comes from their perceptions about the goals of malicious users. Ostriches often believed that the goals and motivations of malicious users were not truly malicious, but instead aimed at showing how smart the malicious user was. Putting the focus on malicious users showing off allowed Ostriches to downplay the severity of the consequences.

Of all the user types, Ostriches had the highest belief in the effectiveness of their safeguards, but this must be looked at alongside the fact that most Ostriches only used the most basic safeguard of having antivirus software installed on the computer. In addition, the protective software Ostriches used was either (1) pre-installed when they purchased 
the computer, (2) built in to the operating system, or (3) recommended by the retailer where they purchased their computer who they considered to be more knowledgeable about security. Ostriches were unlikely to engage in more protective behaviours and instead preferred to ignore threats as much as they could, which was often done by downplaying their susceptibility to threats.

Overall, Ostriches had the lowest levels of threat awareness, knowledge, and perceived self-efficacy. As a result, they generally tried to avoid concern about the dangers by downplaying their susceptibility to threats and the severity of those threats. Overall, this meant that Ostriches either ignored the threats and hoped nothing would happen or they passed the responsibility of protection from threats on to someone else so they could be absolved from not taking more steps to protect themselves. The greatest difficulty with convincing Ostriches of the need to protect themselves is providing evidence that there is a reason to take protective steps, even when past use of the internet has not yielded a catalyst event to prompt better behaviours.

\subsubsection{Mice}

Respondents who engaged in very timid security behaviours can be compared to mice. Mice tend to be wary about how they use the internet, often due to lower levels of trust than their Ostrich counterparts. Both Mice and Ostriches had lower levels of knowledge and awareness of threats. However, Mice tended to be slightly more knowledgeable about the potential risks online than Ostriches. Because of this, Mice tended to take slightly more protective steps than Ostriches, and if they were unsure of how to take these protective steps, they sought someone out who they knew could take the steps for them. Individuals who could be associated with the Mouse user type represented the largest portion of respondents (19/34).

\section{FEATURES}

Mice tended to be among the more trusting respondents in terms of websites and people online. Mice had a tendency to trust people and hope that by trusting them everything would turn out fine. In many cases, Mice were willing to give people, including malicious users, the benefit of the doubt. Mice often favoured describing malicious users in benign terms. Mice also tended to believe that if they did suffer a cybersecurity breach, malicious users were not acting in ways that would cause them or their computers ongoing harm. Mice were slightly more likely than Ostriches to believe that malicious users actively sought 
to cause users intentional harm.

Mice had slightly higher levels of safeguard awareness (3) than threat awareness (2). These respondents implied that they did not have to know how the safeguards worked or exactly what they protected against in order to feel protected, suggesting low to moderate levels of knowledge. However, these same respondents often indicated that they needed to understand how threats worked and what they did before they considered themselves to have high levels of threat awareness. This resulted in Mice having higher levels of safeguard awareness (3) than Ostriches (2), which translated into better security behaviours.

Mice were more aware of the need to use antivirus software than Ostriches were, and their use of antivirus software helped them to feel more secure using the internet because they felt that it provided a wide range of protection against online threats. The use of this software often boosted their confidence that they would not suffer a cybersecurity breach. It also allowed these respondents to feel more comfortable about their security without the need to increase their knowledge since the protective software was used to supplement their existing knowledge.

Mice were unlikely to have experienced cybersecurity breaches in the past. However, they were slightly more likely to have experienced breaches than Ostriches. Some Mice were more 'hopeful' than 'certain' that they had not suffered from breaches, typically because they had experienced what they considered to be the consequences of a breach. Mice were slightly more likely to consider certain symptoms to be indicative of a breach, even without other evidence to support the existence of a breach. However, they often considered themselves to be "lucky" that they had not suffered from breaches in the past.

Although Mice were generally timid and had low to moderate levels of trust (3), threat (2) and safeguard (3) awareness, and prior experience (2), some respondents differed in their levels of knowledge, perceived self-efficacy, security orientation, and reliance. This led to the creation of two sub-types of Mice: House Mice and Field Mice (see Figure 6-4, p. 199). Field Mice (3) were generally slightly more knowledgeable than House Mice (2) and had somewhat higher levels of perceived self-efficacy (House Mice: 1, Field Mice: 3) and security orientation (House Mice: 2, Field Mice: 3). In contrast, House Mice were also characterised by their very high levels of reliance on others (5). Of the 19 respondents who fit into the Mouse user type, five were most closely associated with the House Mouse user type.

Field Mice (3) were more knowledgeable than House Mice (2) and Ostriches (2). In particular, Field Mice were slightly more knowledgeable than House Mice about what threats were and how they could protect themselves. These slightly elevated levels of knowledge had a positive effect on Field Mice's awareness of both threats and safeguards. Despite Field Mice having slightly more knowledge than House Mice, both had similar levels of threat (2) and safeguard (3) awareness. Although House Mice had slightly lower levels of knowledge than 
their Field Mice counterparts, they still considered it important to take steps to protect themselves online. However, because Field Mice were content with the knowledge that they had and did not seek out additional information about threats and safeguards, they tended to only engage in the basic protective behaviours that have long been promoted by security experts. Fourteen of the respondents shared similar features to Field Mice. Although this number seems high, it is not unexpected because as discussed later, the Field Mouse is the type closest to the normal and average user.

Mice who considered themselves to be less knowledgeable than the average user often did not actively seek out information about security because they felt they would not be able to understand the information enough to be able to follow through. Both Mice and Ostriches engaged in similar self-perpetuating cycles whereby individuals felt that they did not have enough knowledge to take more protective steps or to seek out additional information, and as a result did not gain the additional knowledge and confidence the could come from searching for information. This cycle further perpetuated their feelings of not being knowledgeable enough.

Although neither House nor Field Mice had particularly high levels of perceived selfefficacy or security orientation, Field Mice were the more confident of the two in terms their abilities. The slightly elevated levels of these two factors for Field Mice meant that they were more likely than their House Mice counterparts to try to protect themselves because they were confident enough in their skills to try to take steps to alleviate some of their concern about security by engaging in protective behaviours.

House Mice had much higher levels of reliance on other people to protect their computers than Field Mice. House Mice had the highest levels of reliance amongst all the types of users identified. These higher levels of reliance were closely linked to their lower levels of perceived self-efficacy, threat awareness, and safeguard awareness. House Mice knew 
enough about the threats and safeguards to understand that they needed to do something to protect themselves. However, they were also aware of the limitations of what they could do based on their skills. For example:

- I don't know how to do that protection, but I would be aware that you need to. (Int-13)

- I know enough to know that it's important. But that's because I'm married to who I'm married to... But it doesn't mean I know how to personally do things about it. (Int-5)

These features combined in a way that made House Mice more likely to seek out someone they considered to be more knowledgeable about threats. Because they were concerned about their security but did not have enough confidence in their abilities to actually take the steps to protect themselves, they placed the responsibility on people they believed to be more computer savvy.

House Mice tended to depend highly on others to protect them from threats. They often placed the responsibility for preemptively protecting the computer from threats on their spouses or children. If something did go wrong, they expanded who they depend on to remedy the situation to include website owners, banks, or computer experts who they expected to fix their problems. Typical responses from House Mice explained that they did

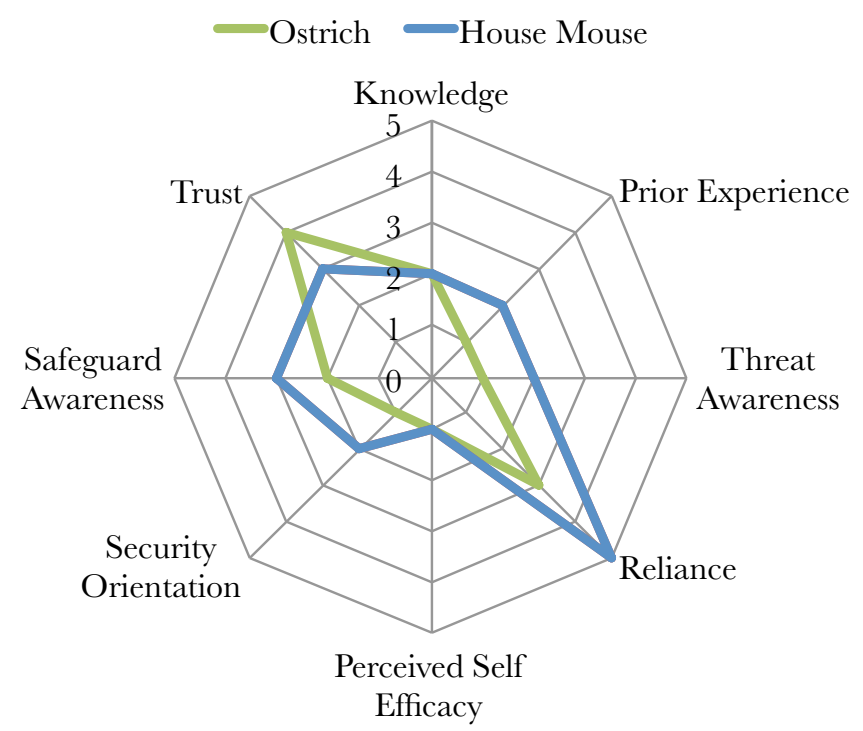

Figure 6-5. Comparison between key features of Ostriches and House Mice not need to worry about protecting their computer because someone else took care of it for them. Their dismissal of security due to their reliance on others could result in a House Mouse being mistaken for an Ostrich (see Figure 6-5). House Mice were more aware of both threats and safeguards than Ostriches. Therefore, although their level of reliance was higher than that of their Ostrich counterparts, House Mice had higher levels of reliance to supplement their lower levels of skill, whereas Ostriches relied on others because it helped them to be 
able to ignore the threat.

\section{BEHAVIOURS}

Overall, Mice engaged in more protective behaviours than Ostriches, but did not take as many protective steps as other types of users, which is discussed later. However, while House Mice often had similar behaviours to Ostriches, Field Mice took more steps to ensure their own security.

The biggest barriers to Mice engaging in safer behaviours were their lower levels of knowledge and what many respondents described as "laziness." Mice were often aware that they were less knowledgeable than they could or should have been. These lower levels of knowledge were often tied to their feelings of laziness because these feelings prevented them from seeking out more knowledge. However, the laziness in regards to seeking out information was underscored by Mice feeling that they did not have enough knowledge to understand what else they could do to protect themselves, even if they knew they should be doing more.

House Mice often tried to protect their security by seeking out people they considered to be more knowledgeable than themselves. However, they did not always ensure that the people they depended on to protect them were aware that they were being relied on. In contrast, Field Mice often undertook the security behaviours they were familiar with and hoped for the best, as one of the interviewees described:

As long as I've got virus software, I just assume my PG is safe. (Int-6)

These kinds of assumptions were common amongst Field Mice who hoped for the best. With these sorts of behaviours, while there was a basic level of security, the respondents in this category were much more prone to making assumptions that the steps they took would keep them safe.

Overall, while Mice tended to engage in more protective behaviours than Ostriches, these behaviours were still often limited. Because of Mice's lower levels of knowledge and perceived self-efficacy, they did not always have enough understanding of what steps they could take to protect themselves, which limited what they did. House Mice shared some similarities with Ostriches, often relying on others to protect their security. Field Mice tended to behave as safe as they knew how, even if their knowledge and awareness was limited. 


\subsubsection{Cockerels}

The phrase "cock of the walk" has been used to describe a person who is proud and thinks that he is better than his peers. Similarly, in the security context Cockerels often tried to show off to those around them their confidence in their ability to protect their computers. Cockerels were distinguished from other user types by their high levels of perceived selfefficacy.

\section{FEATURES}

All Cockerels expressed high levels of confidence (5) in their ability to protect themselves from threats online. For example, two participants discussed how capable they felt of protecting themselves online:

Probably better than average. (Par-2D)

Everyone thinks they're better than average. (Par-2E)

And I am... probably because of work. (Par-2D)

Despite similar outward appearances which were the result of high levels of perceived selfefficacy, there were two types of Cockerels: Cluckers and Crowers (see Figure 6-6). For Cluckers, increased levels of perceived self-efficacy appeared to be influenced by threat awareness (5),

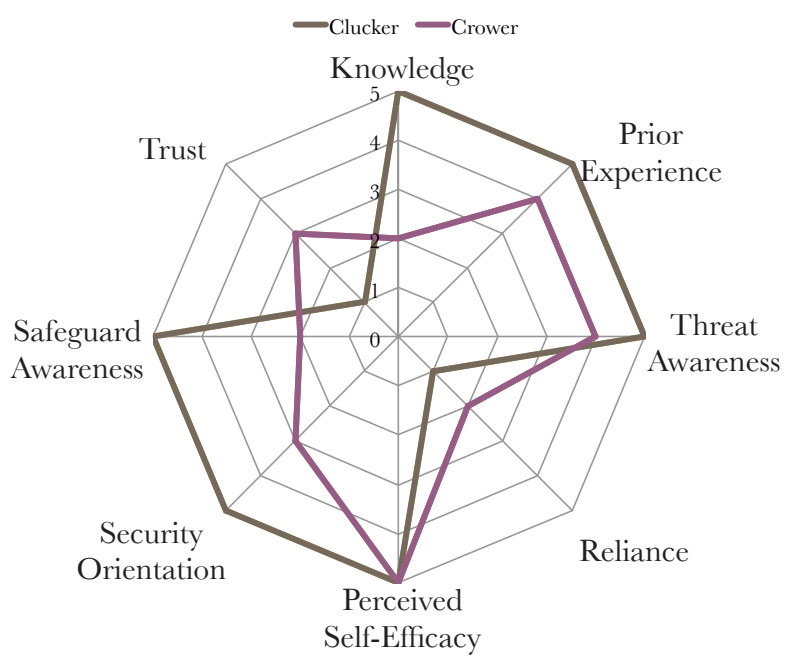

Figure 6-6. Overview of the key features of Crowers and Cluckers prior experience with cybersecurity breaches (5), and users' knowledge (5). Four respondents in this study fit with the features of Cluckers. Crowers were more influenced by their perceived levels of awareness and knowledge as their actual levels of threat awareness (2) and knowledge (2) were lower. Three respondents could be considered as Crowers. In the quote given above, the participant who expressed high levels of confidence in his skills did 
not demonstrate equally high levels of understanding about threats and how he could protect himself.

Similar to how cockerels protect the hens in the farmyard from predators and other cockerels, Cluckers often took what could be considered extreme protective steps to ensure that their computers and data were protected from what they saw as threats. This was due to their elevated levels of threat awareness (5), prior experience (5), knowledge (5), safeguard awareness (5), and security orientation (5) combined with low levels of trust (1) and reliance (1). Thus, although they were often quietly confident in their skills, when asked about their security, they did not hesitate to display their knowledge.

Both Crowers and Cluckers had similar levels of reliance (Crowers: 2, Cluckers 1), threat awareness (Crowers: 4, Cluckers 5), and prior experience (Crowers: 4, Cluckers: 5). However, where Crowers tended to exhibit moderate to high levels of these features, Cluckers demonstrated consistently high levels. Crowers were slightly more reliant (2) on others than their Clucker counterparts (1). This stemmed from their lower levels of knowledge (2) about the steps that they could take to protect themselves, despite the fact that they were confident in their ability to do so if they desired (5). In contrast, Cluckers displayed the lowest levels of reliance (1) because they believed that nobody else was as knowledgeable or as skilled at security as they were. Therefore, they often considered it to be "pointless" to depend on others as it would not provide any additional security.

Threat awareness helped provide Crowers with a baseline of necessary information to support their claim that they had higher levels of knowledge. However, Crowers often mistook their increased threat awareness for increased knowledge. Increased threat awareness often helped Crowers to conceal that their actual levels of knowledge were not as high as they believed them to be. A certain level of threat awareness was needed for Crowers to convince less knowledgeable users that the Crowers were more skilled. When combined with their high levels of perceived self-efficacy, Crowers were able to present a very convincing initial display of their security prowess giving them the appearance of being highly knowledgeable. One of the main characteristics that differentiated Crowers and Cluckers was that Crowers often had lower levels of actual knowledge (2), despite their knowledgeable façade. In contrast, Cluckers tended to have high levels of knowledge (5) which were supported by their high levels of threat awareness (5).

Both Crowers and Cluckers tended to get information about threats from the media which may have accounted for their high levels of threat awareness. However, Cluckers were more likely to actively seek out information about threats, while Crowers tended to wait for the information to be pushed to them. Crowers were much more likely to credit work notifications and the media with their increased threat awareness. The difference between active and passive information seeking may have contributed to the differences between 
their safeguard awareness.

Cockerels tended to be confident that they had previously experienced cybersecurity breaches (5). By having experienced a breach or even a perceived breach in the past, Cockerels had experienced a catalyst event that Ostriches and Mice typically had not. This appeared to be one of the reasons why Cockerels tended have higher levels of threat awareness. Threat awareness also appeared to have a positive effect on respondents' perceived self-efficacy because this awareness helped them know what they need to be wary of.

Despite their higher levels of threat awareness, Cluckers and Crowers demonstrated very different levels of knowledge. Cluckers were often able to describe precise symptoms and even postulate on the causes of past breaches which suggested higher levels of knowledge (5). However, Crowers tended to describe the symptoms of past breaches in much broader terms. Crowers often indicated their computer started "acting funny" or took its slowing down as signs that it was infected with malware, suggesting lower levels of understanding (2). While being able to identify the causes of a security breach is not the sole way that a user's knowledge and skill can be judged, it did provide insight into it.

Crowers' lower levels of actual knowledge (2) appeared to have an influence on their safeguard awareness. One reason why Crowers may have had lower levels of safeguard awareness was because their levels of knowledge were lower. As a result, they often waited for information to be pushed to them. In particular, Crowers tended to rely heavily on information from work sources. Dinev \& Hu (2007) suggested that users' safeguard awareness may be lower if they are not involved in protecting their work computers since the process of securing the computer may be essentially invisible to them (p. 388). Thus, Crowers may have assumed that their home computer was similarly protected to their work computer. Further to this, they may also have assumed that they did not need to know any more about safeguards because they already assumed they were protected.

In contrast, Cluckers tended to seek out information and had higher levels of knowledge. One result was that Crowers were more likely to not only seek out information on ways to prevent and remedy threats, but they were also more likely to have understood and implemented those safeguards. These information seeking behaviours appeared to be at least partially responsible for Cluckers undertaking many more safeguards than Crowers. Crowers' lower levels of knowledge (2) often contributed to their lower levels of safeguard awareness (2) because they tended to assume that threat awareness was equivalent to an understanding of how to protect themselves. Therefore, while they tended to have higher levels of threat awareness, their safeguard awareness was lower since in order to understand safeguards they would have needed to actively seek out information about steps they could take to protect themselves.

Cluckers were very concerned about their security almost to the point of being 
paranoid. This often stemmed from very negative experiences in their past. Crowers often professed to be very concerned about their security, but their comments suggested that their actual levels of concern were much lower. These lower levels of concern often stemmed from justifying why they did not consider the threats to be dangers. Most often this was because they felt that they were either unlikely to have to face a threat because it had not happened in the past or because they did not consider the consequences to be very severe because they did not believe that they stored valuable data on their computers.

Cluckers tended to have very low levels of trust (1) in both people and websites. As a result, these low levels of trust combined with their high levels of knowledge and awareness at times resulted in feelings of paranoia. Often Cluckers stated that they did not trust anyone or anything online, even going so far as to distrust communications from friends and family. These feelings of self-described paranoia appeared to carry through into Cluckers perceptions of malicious users as well, often believing that malicious users were actively targeting them.

Overall, Crowers had similar levels of knowledge (2) and trust (3) to Mice. Unlike Cluckers, Crowers tended to find reasons to dismiss the threat posed by malicious users. One way that Crowers did this was by suggesting that they did not fit the profile for being a victim of a malicious user, for example, because they were not rich, did not use a Windows PC, or did not have any valuable information on their computers. This was similar to the way that Ostriches justified why they did not worry about threats. However, Crowers were more likely than Ostriches to ascribe negative goals to malicious users.

\section{BEHAVIOURS}

Crowers and Cluckers tended to engage in different types of security behaviours. Crowers behaviours were predominately ego defensive. Crowers tried to display their high levels of knowledge in order to distract from their potentially less secure behaviours. In contrast, Cluckers engaged in many security behaviours, often to the extent that these behaviours would diminish the usefulness of the computer.

Unlike Crowers who had a gap between their perceived knowledge, actual knowledge, and awareness which resulted in less secure behaviours, Cluckers' perceptions were often accurate and reflected an understanding of the potential risks online. As a result, Cluckers engaged in more protective behaviours overall. Of all the user types, Cluckers were the least affected by barriers to engaging in safer behaviours. Cluckers often indicated that this was because they were more highly concerned about their security overall. This often meant that they considered the risks to be too high to act in an unsafe manner. Cluckers often considered security be more important than the effort it would take to overcome the 
barriers. As a result, Cluckers often dismissed the barriers as being unimportant because they considered good security to be part of their lives both online and offline.

While Cluckers' features seemed likely to lead to ideal security behaviours, their concern often led to a conscious decision to use the internet less. Cluckers were more likely than other users to believe that malicious users would specifically target them in order to steal or destroy their data. When combined with their low levels of trust in people and websites, it had the potential to create feeling of paranoias. The result of this paranoia was that Cluckers tended to avoid any activity they deemed "risky" and the steps they took to protect themselves made it more difficult for them to use the Internet.

The activities that Cluckers deemed risky varied from user to user. However, the most common activities that users avoided were: using online banking, shopping online, interacting with strangers, and visiting unknown websites. Cluckers often worried that someone would steal their login information and proceed to defraud them. As a result, Cluckers frequently opted to avoid using the services at all, rather than trying to identify a way to use them safely. The increased concern and "paranoia" often appeared to be linked to their previous experiences with cybersecurity breaches. Often Cluckers were certain they had experienced past cybersecurity breaches, and because of their high levels of knowledge, skill, and awareness frequently either knew or were able to make an educated guess about the causes of the breaches. As a result of those breaches and the knowledge of how they were caused, Cluckers tended to try to avoid similar situations to those that had caused breaches in the past. Those situations and other similar situations were then more likely to be considered "risky" activities to be avoided by Cluckers.

Many Cluckers who considered themselves to be "extremely paranoid" indicated that they would use false identities to register on websites, then used those identities to determine if the website had sold their information to a third party or to confirm if the communication was coming from who they expected it to be from. In many cases, these actions seemed to confirm that the users were highly concerned about the potential for a breach to happen. While these steps offered some protections in terms of communications, they also required more time to implement and maintain while not offering as much protection as the person may have hoped for. The use of false identities further shows how Cluckers had very low trust in not only people online, but also in the reliability of security software, and as a result, these users often showed this distrust through their actions.

Crowers often engaged in similar behaviours to Mice and made excuses for why they took fewer steps to protect themselves. Crowers were more likely to create excuses for their behaviours because they did not have the actual knowledge required to protect themselves from threats. Therefore, they may have used other unrelated excuses as a defence mechanism. Crowers were likely to say that the reason they did not take more 
steps to protect themselves was because they were not the intended targets of malicious users. Often this perception appeared to be caused by a belief that they did not fit the characteristics of who they believed malicious users should target. One example of this was when Crowers suggested that it would not be financially viable for a malicious user to target them. These justifications by Crowers were similar to how Mice tended to rationalise why they did not take more steps to protect themselves. The main problem that arose from these assumptions was that such assumptions were likely to be inaccurate. Malicious users often indiscriminately target individuals in order to run attacks against corporations (see Culnan et al., 2008). Therefore, although these justifications helped to allow both Crowers and Mice to convince themselves that there was less of a need to engage in protective behaviours, they may have been putting themselves at risk due to an incomplete understanding of how malicious users utilise threats.

Crowers often considered themselves to be better protected than their peers, regardless of the steps they took. This may have been due to an optimistic bias (see for example Rhee et al., 2005). If Crowers believed that they were already sufficiently protected, they may not have seen any reason to take additional steps. However, the danger was that if Crowers did not fully understand threats and how they worked, they might not have been able to accurately judge whether they were better protected than their peers.

Crowers often stated that external barriers had the greatest effect on their behaviours. In contrast to Mice who tended to be more hindered by internal factors, Crowers tended to focus on the financial and time costs associated with being secure. For Crowers, a cost-benefit analysis often appeared to take place where, for example, the Crower would indicate that they chose not to engage in a particular protective behaviour because it took too much time. While Crowers tended to indicate that they were mostly influenced by external barriers, the way that Crowers favoured the external barriers appeared to have been a way to deflect from the effect of internal factors on perceived danger. As noted previously, Crowers often engaged in behaviours that would allow them to protect their egos, such as by boasting about their security skills. As a result, they were more likely to have indicated that external barriers were more likely to prevent them from engaging in safer behaviours. The reason Crowers seemed to place more emphasis on the external barriers may have been because of their high levels of perceived self-efficacy. If Crowers were to admit that internal factors, such as a lack of knowledge, were stopping them from being safer online, it could have destroyed the façade they were trying to project. However, the way that Crowers favoured external barriers was one of they ways they are distinguished from Mice.

Like Mice, Crowers were more likely to engage in unsafe behaviours due to naïve assumptions and the justifications for why they did not take more steps to protect themselves. For example, Crowers were more likely to buy something online from a smaller e-tailer than 
Cluckers because they did not believe that there was any reason why malicious users would target either their transaction or the e-tailer. Similarly, Crowers were less likely to update their computer or antivirus software immediately upon expiry because they did not think that their computers held anything of value or that they would be targeted by malicious users. Similar to Mice (see section 6.5), this suggests that Crowers were making assumptions, potentially based on their lower levels of knowledge about why and how malicious users would target them.

Despite the fact that Crowers appeared to share many similar features with Cluckers rather than Field Mice, Crowers' behaviours tended to be most similar to Mice. Crowers engaged in more basic protective behaviours (such as the use of antivirus software, not opening email attachments, and not visiting "risky" sites) and engaged in risky behaviours naïvely rather than intentionally. Thus, one of the biggest challenges for improving Crowers' behaviours is in finding a way to improve their knowledge without damaging their self efficacy.

Overall, Cockerels spoke highly of their abilities to protect themselves online. However, because Cockerels could be either Crowers or Cluckers, it was necessary to look beyond their crowing to determine if it was an accurate portrayal of their skills. Thus, the first challenge in improving Cockerels'behaviours is determining whether they are Cluckers or Crowers. Once it is determined whether a user is a Crower or a Clucker, each has its own challenges associated with improving security behaviours. With Crowers, the main challenge is to overcome their strong self confidence in order to improve their actual knowledge without destroying that confidence in their skills. With Cluckers, the challenge is not as much related to improving their behaviours, but finding a way for them to reconcile their concerns with safer ways to use services that may be beneficial to them.

\subsubsection{Coyotes}

In Native American mythology, the coyote has often been portrayed as being clever and doing whatever it takes to further his agenda. He has been used as an example of how "poor behavior may benefit you in the short term, but in the long run your tricky, selfish gain will not serve you" (California State Parks, 2009). Similarly, Coyotes often pushed the boundaries and took risks with their security which may have provided short term rewards, but created more long term dangers. Overall, Coyotes' distinguishing behaviour was that they tended to knowingly take risks online often to obtain short term rewards. 


\section{Features}

Coyotes (see Figure 6-7) had moderate to high levels of security orientation (4), threat awareness (4), safeguard awareness (4), knowledge (4), prior experience (4), and perceived self-efficacy (4). Their knowledge, combined with their previous experiences with cybersecurity breaches appeared to contribute the

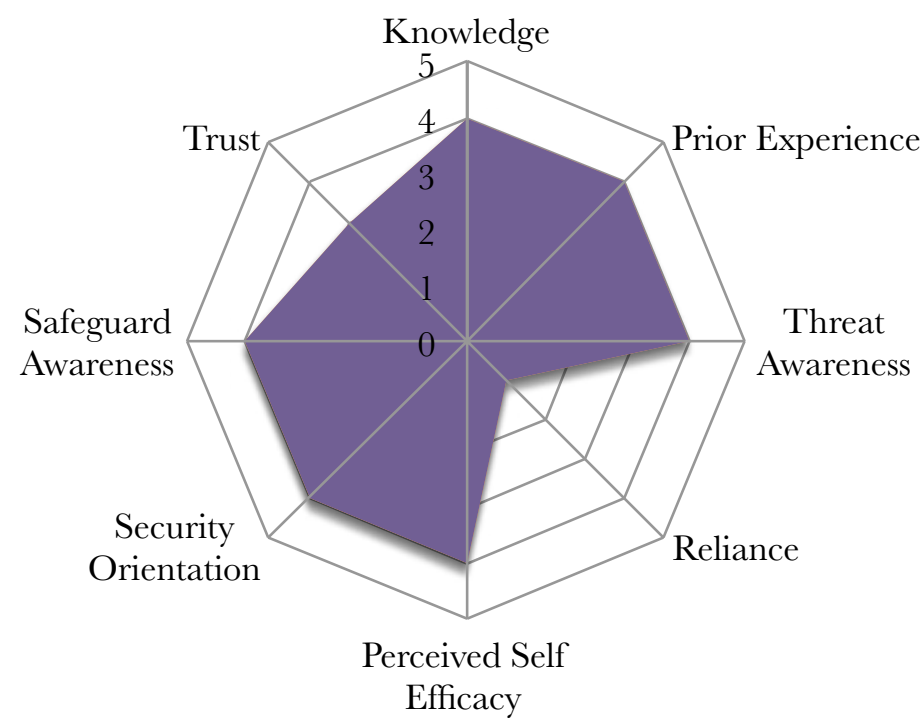

Figure 6-7. Overview of the key features of Coyotes most to their risk taking behaviours. Three of the respondents in this study had features closely associated with Coyotes. In many ways, Coyotes had similar features to Cluckers, but were more trusting overall (Coyotes: 3, Cluckers: 1). Like Cockerels, Coyotes often stated that they had experienced at least one cybersecurity breach. Coyotes described these breaches in more detail than their Crower counterparts, and in many cases, the Coyotes also believed they knew what had caused the breaches. However, their descriptions and understanding were rarely as detailed as those of Cluckers.

Although Coyotes were aware of the potential risks that they faced online, they often knowingly chose to engage in behaviours that could have been considered risky. One reason why Coyotes were less concerned about the risks of these activities was because in the past they had successfully remedied the consequences of breaches they had experienced. One of the respondents who exemplified the features of a Coyote stated:

You know, you do something dumb and it means that you lose your files or you have to reinstall the operating system or whatever, and that's a real pain. And I guess mostly it's just that I don't want to have to deal with that again. (Int-7)

Although this Coyote indicated that she considered her actions to be "dumb" she also indicated that she was aware of the risks of her actions. She indicated throughout her answers that she'd managed to recover from breaches in a way that she was satisfied with, which increased her confidence that she would be able to deal with a breach if it arose in the future, even though she preferred not to. Bandura (1977) found that "after 
strong efficacy expectations are developed through repeated success, the negative impact of occasional failures is likely to be reduced" (p. 195). Because of Coyotes' past success in remedying the consequences of breaches, they often assumed that they would be able to remedy the effects of future breaches. Furthermore, they were also confident in their ability to protect themselves online because they had been able to do so in the past. In addition, the successes that Coyotes experienced, may also have further contributed to the amount of effort they put into protecting themselves online (see for example, Bandura, 1977), and increased their concern over security.

Coyotes'levels of trust (3) tended to be higher than Cluckers (1), similar to that of Crowers (3) (see Figure 6-8). Crowers tended to trust people online because often assumed that they were unlikely to be targeted. In contrast, Coyotes tended to extend a moderate amount of trust to both people and websites, preferring to give them the benefit of the doubt

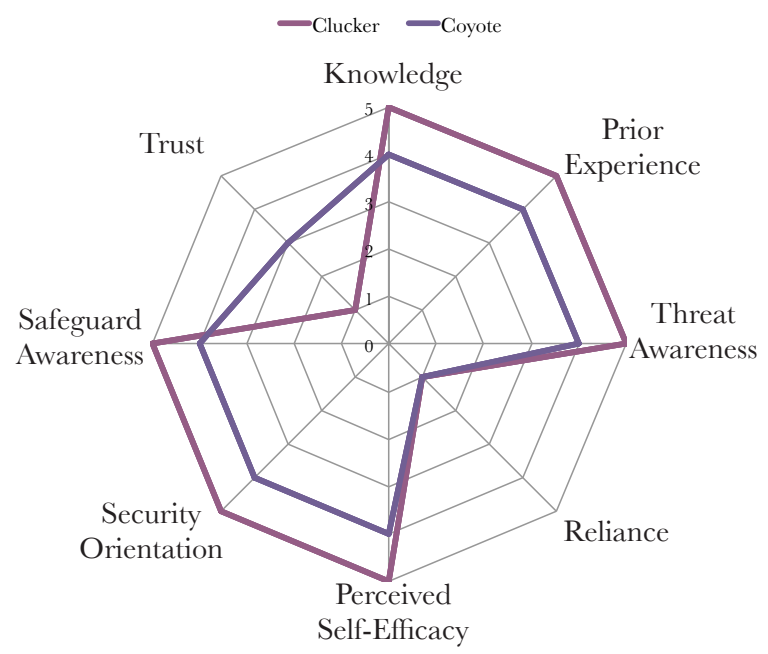

Figure 6-8. Comparison between the key features of Coyotes and Cluckers rather than immediately dismissing them. This appears to be partially due to the confidence that Coyotes had in their ability to remedy cybersecurity breaches. Because Coyotes often felt that they could remedy any issues that arose, they may have been more comfortable with extending their trust to people and websites they were not as familiar with.

Unlike Cluckers who tended to believe that malicious users were actively seeking to attack them and Crowers who tended to justify why they would not be targeted, Coyotes tended to believe that there were malicious users out there who would target them. However, they were not as concerned about being specifically targeted as they were about being targeted as part of a more widespread attempt. Overall, they were more likely than Mice, Ostriches, and Crowers to consider malicious users' actions as malicious. However, they were not as concerned as Cluckers. One reason for this appeared to be due to the increased knowledge and awareness of threats that Coyotes had. Often Coyotes took it upon themselves to seek out relevant information about threats. Thus, their knowledge about the types of threats and the way that threats target people tended to be more accurate than other user types. 


\section{BEHAVIOURS}

Coyotes' behaviours tended to be more secure than those of the Ostriches, Mice, and Crowers. Coyotes tended to take more than just the basic protective steps. However, Coyotes did not take as many steps to protect themselves as Cluckers despite sharing some similar characteristics. Instead, Coyotes often preferred to weigh the benefits of engaging in a potentially unsafe behaviour against the perceived drawbacks of using safeguards. This deliberate risk taking distinguished Coyotes from the other types of users identified in this study. Coyotes were the only user type who admitted that they knowingly took risks online. Mice, Ostriches, and Crowers occasionally took risks online. However, they tended to either do so naïvely or rationalise their risk taking as not being risky. In contrast, Coyotes tended to acknowledge the risk and knowingly engage in behaviours that could be considered risky.

When Coyotes' knowledge was combined with their belief that they could fix any problems that arose, it appeared to make them more likely to take risks. At times this made Coyotes appear similar to Crowers. However, the key difference between Crowers and Coyotes was that the Coyote typically had the skill and knowledge to remedy many of the immediate problems that would have been caused by a breach. In contrast, the Crower typically did not have the safeguard awareness or knowledge that a Coyote did (see Figure 6-8, p. 210). Thus, while Crowers tended to take naïve risks as a result of their confidence in their skills, Coyotes more often knowingly took risks where they considered the potential consequences to be acceptable.

This did not mean that Coyotes engaged in many risky behaviours. Instead, Coyotes tended to only engage in risky behaviours where they perceived a clear benefit for not being as safe as they could have been. Unlike Mice who appeared to be more likely to take risks with illegal file sharing (e.g. mp3s movies, software, etc.), Coyotes risks tended to be based on their awareness of what could happen. As a result, most Coyotes tended to shun file sharing behaviours because they considered the risk to be too high. In contrast, the risky behaviours Coyotes engaged in related more to ways that they could achieve more from their online experience. For example, Coyotes were the most likely user type to use online banking and bill payments. Mice were often too worried about the potential consequences associated with using an unfamiliar technology, and Cluckers considered doing so to simply be unsafe. Coyotes were more likely to trust people online, shop online, visit unknown websites, and download software than other types of users. However, Coyotes also engaged in more security behaviours than Ostriches, Mice, and Crowers.

Unlike Crowers who often believed that they could "handle" security breaches since they had not experienced dire consequences in the past, Coyotes often believed that because they had fixed problems before they would continue to be able to do so in the future. 
The danger in this belief was that Coyotes often considered the consequences of threats to be less serious than they actually were. This may have been because Coyotes were more prone to focusing on the short term gains associated with ignoring certain aspects of their security. Returning to the example of a breach in which a user's bank account details are disclosed, a Coyote was more knowledgeable than a Mouse about how to remedy the breach on the computer. However, the Coyote would still have to address additional consequences, such as damage to their credit rating or proving to the bank that they did not conduct the questionable transactions. These were steps that Coyotes did not often acknowledge, while more cautious users did acknowledge these possible issues even if they did not know exactly what they would have to do to remedy them.

The two main factors which prevented Coyotes from engaging in better behaviours were laziness and a desire to keep doing something that utilising a safeguard would stop them from doing. In many cases it appeared that the greatest barrier was that engaging in safeguards would make using the Internet more difficult for Coyotes' purposes. Thus, while laziness was often mentioned as a reason for not being as safe online, it was not as influential for Coyotes as it was for Mice and Crowers.

Overall, Coyotes tended to engage in slightly more secure behaviours than the average user. However, they were also the most likely of any of the user types to knowingly take risks online. Because Coyotes tended to be aware of their risk-taking nature, it may be more difficult to convince them to change. The main reason for this was because they tended to consider their risks as calculated based on their knowledge, perceived self-efficacy, and awareness. Therefore, in order to improve Coyotes' behaviour they would have to be convinced that the benefits of engaging in safer behaviours outweighed the benefits of engaging in less safe behaviours.

\subsubsection{Dark Horses}

The phrase "dark horse" has been used to describe someone who is an unexpected success, much like the individuals in the final group of respondents. These respondents tended to have low levels of perceived self-efficacy combined with unexpectedly high levels of knowledge. The discrepancy between respondents' perceived self-efficacy and knowledge is what distinguishes them from the other types of respondents in this study. What makes Dark Horses an unexpected success in their use of safeguards is that based on their low levels of perceived self-efficacy it would be expected that their security behaviours would be similar to Mice. However, on further examination, their knowledge clearly distinguished them when they spoke about the protective steps they took. 


\section{Features}

In contrast to Cockerels who tended to have very high levels of perceived self-efficacy, Dark Horses were characterised by their low levels of perceived self-efficacy (see Figure 6-9). These lower levels of perceived self-efficacy often led Dark Horses to believe that they were less aware of threats and less knowledgeable about security. However, while their perceived knowledge and awareness were low, their actual knowledge and awareness were as good as and at times better than Cluckers. This lack of confidence by Dark Horses appears to stem from their past negative experiences with cybersecurity breaches. This group of users represented the smallest proportion of respondents in the study with two respondents fitting the features of Dark Horses.

Similar to Cockerels and Coyotes, Dark Horses had often experienced a cybersecurity breach where they experienced some type of loss (e.g. financial loss, data loss, etc.). What distinguished Dark Horses from other types of users was how they reacted to those breaches. Cockerels tended to explain away breaches as malicious users being smarter than them and Coyotes tended to accept that breaches were inevitable. However, Dark Horses were more likely than other types of users to place the blame for cybersecurity breaches on their own behaviours, for example:

It's definitely something that I have done. In terms of hacking into my password, my account (Int-18)

Unlike Ostriches, Field Mice, and Crowers who tended to avoid taking responsibility for their security, Dark Horses often took responsibility for the cause of the breach. This increased sense of responsibility for causing breaches appeared to contribute to their lower levels of perceived self-efficacy.

Dark Horses who had experienced breaches in the past often demonstrated lower confidence in their own skills, attributing their lack of skills to their failure to take sufficient 
steps to protect themselves online. Dark Horses often described at least one negative incident that prompted them to change their security behaviours, though some described several. Bandura (1977) found that "repeated failures lower [efficacy expectations] particularly if they occur early in the course of events" and that "the effects of failure on personal efficacy therefore partly depend on the timing and the total pattern of experiences in which the failures occur[ed]" (p. 195). This helps explain why Dark Horses who had experienced breaches often had lower confidence in their ability to protect themselves in the present. Furthermore, it helps explain the difference between how Dark Horses perceived their knowledge and awareness in comparison to what they demonstrated.

Despite perceptions by Dark Horses that they lack the awareness and knowledge required to protect themselves online, they often had levels of knowledge and awareness equal to and in some cases greater than Cluckers, who were among the most knowledgeable types of users. This raised the question of why Dark Horses were so knowledgeable despite statements to the contrary. Many Dark Horses worked in jobs where they were required to have some level of technical skill, often either studying or working in a technology-based role. As a result, the skills gained through their education and work experience were often applied in their home environment. While Crowers also credited their work experience with increasing their knowledge, in most cases they only demonstrated higher levels of threat awareness. The main reason for the difference between the effect of work and education on Crowers and Dark Horses stems from the type of work that Dark Horses were involved in. For Crowers, technology supported their work. In contrast for Dark Horses, technology was often an integral part of their job as they were often responsible for repairing or preventing problems.

Dark Horses' knowledge was closely related to their awareness of threats and safeguards. Similarly to Cockerels and Coyotes, Dark Horses had moderate to high levels of threat awareness and they were often confident in this assessment. However, when examining their safeguard awareness, once again a gap between their perceived and actual awareness emerged. Unlike threat awareness where the individual does not necessarily have to take any actions, safeguard awareness often requires individuals to seek out information about the steps they can take and to understand them. Therefore, while Dark Horses' lower levels of perceived self-efficacy did not diminish their threat awareness, it did appear to contribute to a lower level of perceived safeguard awareness. Dark Horses often believed that they could not effectively protect their computer from threats, often because they felt there were more protective steps out there that they were unaware of that would do a better job than they were. 


\section{BEHAVIOURS}

Despite beliefs by Dark Horses that they were unable to protect their computers, they often undertook some of the most comprehensive security behaviours of the identified user types in this study. Often, Dark Horses identified and explained many complicated behavioural and technological safeguards that they could use to protect themselves. Even when they did not necessarily undertake all the steps they identified, the safeguards they utilised were much more advanced than other types of users, including Cluckers. Thus, although lower levels of perceived self-efficacy contributed to Dark Horses' beliefs that they were unable to protect their security online, their actual knowledge indicated that they were not only capable of doing so, but often actually did so.

In many ways, Dark Horses were the opposite of Crowers (see Figure 6-10). Where Crowers had low to moderate levels of knowledge and safeguard awareness, Dark Horses had high knowledge and safeguard awareness. Similarly, where Crowers had high perceived self-efficacy, Dark Horses' perceived self-efficacy was low. Finally, where Crowers often took minimal security precautions, Dark Horses had some of the most comprehensive security behaviours of

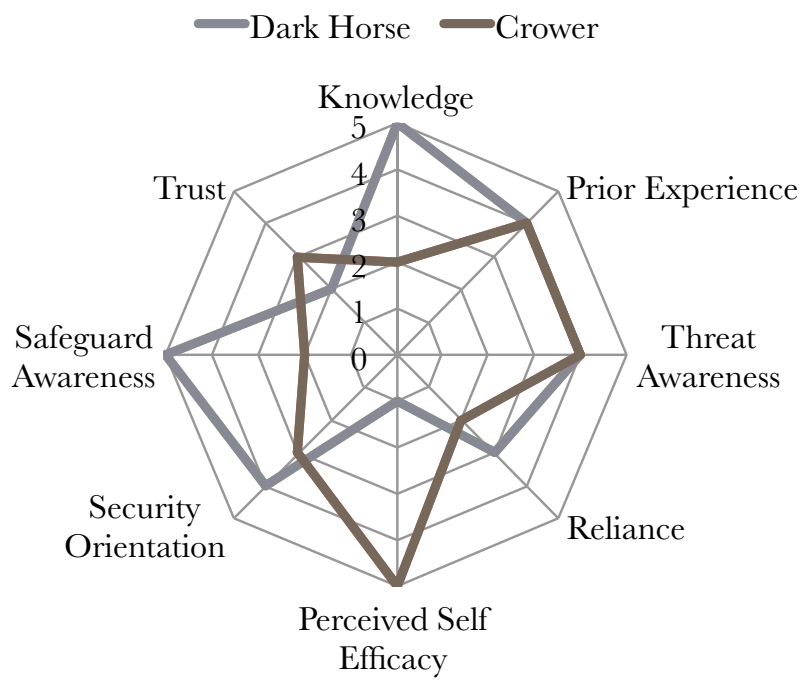

Figure 6-10. Comparison between the key features of Dark Horses and Crowers all of the users in this study.

Dark Horses were frequently dismissive of the efficacy of the security steps they took. Often they believed that the behaviours they were engaging in were not comprehensive enough to offer the necessary protection from online threats, such as:

If someone's out to get me, they can get me, so why go through all this weirdness...if I wanted to $100 \%$ bullet proof the thing, I would have to go through a series of learning which I'm not ready to do at this stage. (Int-18)

Many Dark Horses were concerned that they could not achieve complete protection from threats. Despite the extensive security steps taken by Dark Horses, their beliefs about the effectiveness of safeguards were often lower than average. When looked at in relation to 
their past experiences with cybersecurity threats along with their perceived self-efficacy, it appeared that these factors contributed to Dark Horses focusing on what security measures could not do, rather than what they could do. Therefore, it appeared that the greatest hindrance to Dark Horses doing more to protect themselves online stemmed from their lower levels of perceived self-efficacy.

In many cases, Dark Horses used the acknowledgement of what they considered their shortcomings to describe the steps they knew they could take but did not. In comparison to other types of users, Dark Horses were able to identify more ways to protect themselves than Cluckers. The increased awareness of safeguards appears to be related to Dark Horses' higher level of awareness of threats and knowledge. Despite the knowledge of what Dark Horses could do to protect themselves, there was often a gap between what they considered "best practices" and what they actually did to protect themselves.

Even though Dark Horses tended to take more steps to protect themselves, they often did not think they were doing enough. Dark Horses, while not hindered by technological reasons, were more often hindered by internal barriers, such as feelings of laziness and a lack of confidence in the effectiveness of the safeguards. In contrast, Cluckers generally did not let anything prevent them from engaging in what they considered to be the safest behaviours they were aware of. Despite a lack of confidence in their own abilities contributing to their perceived failure to protect themselves, their increased level of knowledge along with the decreased self efficacy often appeared to have the result of Dark Horses being less concerned over the financial cost barrier of using protective software compared to Crowers and Mice.

One result of Dark Horses' lower confidence in their skills was that they tended to engage in fewer risky behaviours online. Their lower levels of perceived self-efficacy fostered a feeling of concern about what could happen that Dark Horses wished to avoid. In some cases, Dark Horses began to experience elevated levels of concern which led to feelings of paranoia, similar to what was often seen with Cluckers. However, while Cluckers tended to be more concerned that malicious users were actively out to get them, Dark Horses tended to be more concerned about doing something that could have caused a breach.

Despite beliefs by Dark Horses that they were not particularly knowledgeable, they still tended to utilise both basic and advanced protective steps. Of all the types of users, Dark Horses were the most likely to engage in protective behaviours in addition to technological safeguards (e.g. antivirus and firewalls). In addition, their behaviours tended to require more knowledge to carry out. These behaviours provided further support for the existence of a gap between Dark Horses' perceived and actual levels of knowledge, skill, and awareness.

One result of this gap was that Dark Horses, like their Ostrich, Mouse, and Crower counterparts, needed a catalyst event. Where Dark Horses differed was that rather than needing a catalyst event to prompt starting to protect themselves (like Ostriches), they instead 
need a catalyst event to prompt better behaviours (like Field Mice). Although Dark Horses typically engaged in safe behaviours online, including avoiding what they considered risky activities, they still often expressed sentiments that they needed to be doing more. This seemed to be closely related to the idea that Dark Horses did not consider their safeguards to be comprehensive enough to offer full protection. Overall, most of the behaviours that Dark Horses engaged in appeared to offer comprehensive protection from threats as they tended to use both behavioural and technological safeguards.

Overall, Dark Horses' characteristics were similar to Cluckers in all but their self efficacy. Dark Horses' lower levels of self efficacy seem to be related to other characteristics and security behaviours. This presents the first challenge for Dark Horses, which is accurately identifying them. Because of their lower levels of self efficacy, Dark Horses may be more prone to consider themselves as Mice or even Ostriches. Similarly, Crowers and even some Mice may consider themselves as Dark Horses because of a naïve optimism, whereby they believe that they are better protected from online threats than their peers (see for example Rhee et al., 2005). Thus, while it can be useful to look at users' behaviours by categorising them into types based on their characteristics, it is also necessary to look at what users actually know and do, rather than relying on their beliefs.

The main challenge associated with improving Dark Horses' security intentions is not in improving what they do to protect themselves online. Typically, Dark Horses tended to take more steps to protect themselves than other types of users. However, they were still hindered by the perceived barriers to doing so. Thus, the second challenge is to improve their confidence and self efficacy. The result of doing so may be two-fold. First, by improving their self efficacy it may help them overcome the barrier of laziness which could further improve their behaviours. Furthermore, as Dark Horses tended to be in households with Mice, an improvement in their self efficacy may have a follow-on effect on other users in the household. If Dark Horses are more confident in their skills, they may be more willing to share the information they have with others in their household. As a result, they may start taking more steps to protect their shared computers more cooperatively rather than unilaterally, which may in turn help improve the knowledge, skills, and awareness of other users in the household.

\subsubsection{Summary of User Types}

Each of the different types of users identified in this study had several key features and security behaviours that could be used to identify and distinguish them from the other types of users (Table 6-1, p. 218). The value in identifying these user types is as a starting point for determining how users' behaviours can be improved. By looking at the 
main strengths and weaknesses of each user type along with their behaviours, it is possible to create a more targeted approach to improving security, as will be discussed further in Chapter 7.

Table 6-1. Summary of user type characteristics and behaviours.

\begin{tabular}{|c|c|c|c|}
\hline \multicolumn{2}{|c|}{ User Type } & Summary of Characteristics & Summary of Behaviours \\
\hline \multicolumn{2}{|c|}{ Ostrich } & $\begin{array}{l}\text { - Low knowledge } \\
\text { - Low awareness } \\
\text { - Low perceived self-efficacy } \\
\text { - Low security orientation }\end{array}$ & $\begin{array}{l}\text { - Few security precautions } \\
\text { taken. } \\
\text { - Ignore security threats in the } \\
\text { hope they will go away. }\end{array}$ \\
\hline \multirow[t]{2}{*}{ Mouse } & House & $\begin{array}{l}\text { - Low to moderate knowledge } \\
\text { - Low to moderate awareness } \\
\text { - Moderate trust } \\
\text { - High reliance } \\
\text { - Low perceived self-efficacy } \\
\end{array}$ & $\begin{array}{l}\text { - Depend highly on other } \\
\text { people to protect their security. } \\
\text { - Know enough to be worried } \\
\text { about their security, but not } \\
\text { enough to protect it themselves. }\end{array}$ \\
\hline & Field & $\begin{array}{l}\text { - Moderate knowledge } \\
\text { - Low to moderate awareness } \\
\text { - Moderate trust } \\
\text { - Moderate reliance }\end{array}$ & $\begin{array}{l}\text { - Know enough about security } \\
\text { to try to take steps to protect } \\
\text { themselves. } \\
\text { - Do what they know and hope } \\
\text { for the best. }\end{array}$ \\
\hline \multirow[t]{2}{*}{ Cockerel } & Crozerer & $\begin{array}{l}\text { - Low to moderate knowledge } \\
\text { - Low to moderate awareness } \\
\text { - High perceived self-efficacy } \\
\text { - Moderate security orientation }\end{array}$ & $\begin{array}{l}\text { - Profess their security prowess } \\
\text { loudly. } \\
\text { - Know little about what steps } \\
\text { to take to actually protect their } \\
\text { security. }\end{array}$ \\
\hline & Clucker & $\begin{array}{l}\text { - High knowledge } \\
\text { - High awareness } \\
\text { - Low trust } \\
\text { - High security orientation } \\
\text { - High perceived self-efficacy }\end{array}$ & $\begin{array}{l}\text { - Very distrustful online. } \\
\text { - Lack of trust hinders ability to } \\
\text { use the internet to the fullest. }\end{array}$ \\
\hline \multicolumn{2}{|l|}{ Coyote } & $\begin{array}{l}\text { - Moderate to high knowledge } \\
\text { - Moderate to high awareness } \\
\text { - High perceived self-efficacy } \\
\text { - Low reliance } \\
\text { - Moderate trust }\end{array}$ & $\begin{array}{l}\text { - Know the risks, but take them } \\
\text { if there is a big enough pay off. } \\
\text { - Confident in their ability to fix } \\
\text { problems. } \\
\text { - Takes most precautions until } \\
\text { there is a reason not to. }\end{array}$ \\
\hline \multicolumn{2}{|c|}{ Dark Horse } & $\begin{array}{l}\text { - High knowledge } \\
\text { - High awareness } \\
\text { - Low to moderate trust } \\
\text { - High security orientation } \\
\text { - Low perceived self-efficacy }\end{array}$ & $\begin{array}{l}\text { - Take many complex } \\
\text { precautions (similar to Cluckers). } \\
\text { - Feel that their security will } \\
\text { never be good enough, thus } \\
\text { resigned to the possibility of } \\
\text { breaches. }\end{array}$ \\
\hline
\end{tabular}




\subsection{Comparison of Security User Types}

In this section, the types of users will be compared based on some of their key features. By comparing some of the key features of the user types, it is possible to see the similarities and differences of them. Comparing the average respondent to the perceived normal user, it is possible to see that respondents' perceptions of the normal user were different from the user types that emerged in the study. This suggested a somewhat distorted view of how respondents believed others approached security. User types with high and low levels of perceived self-efficacy and knowledge are compared to help draw conclusions on the effects of each of these factors in relation to users' security behaviours. Finally, the user types that engaged in the most and least numbers of protective behaviours are compared.

\subsubsection{The Average Respondent and the Perceived Normal User}

Compared to how respondents' described what they believed to be the normal user, this "perceived normal user" did not match any of the identified user types (see Figure 6-11, p. 220). While the perceived normal user was somewhat similar to the Field Mouse user type, several features differentiated the two types. In particular, the perceived normal user was less likely to have experienced a security breach, and less concerned about security. In addition, the perceived normal user was considered to be more knowledgeable about security, trusting of people and sites online, and have moderate to high levels of threat awareness.

When the perceived normal user was compared to the average respondent (see Figure 6-12, p. 220), there were notable differences between their features. The average respondent was identified by tallying the results of all individuals involved in the study to obtain the mathematical average. The result was the "average respondent" which could be compared to the perceived "normal user" that was identified by respondents and discussed in section 6.3. The perceived normal user still had higher levels of trust, knowledge, and security orientation, as well as a lower likelihood of having experienced a security breach in the past and lower levels of concern about security. This suggested that there was a difference between how individuals perceived the normal user compared to what the reality of the average user appeared to be. Reasons for this are discussed further in Chapter 7. 


$\begin{array}{lllll}\square \text { "Normal" User } & \square \text { Ostrich } \\ \square \text { Crower } & \square \text { Clucker } \quad \square \text { Couse Mouse } \quad \square \text { Field Mouse } \\ \square \text { Coyote } & \square \text { Dark Horse }\end{array}$

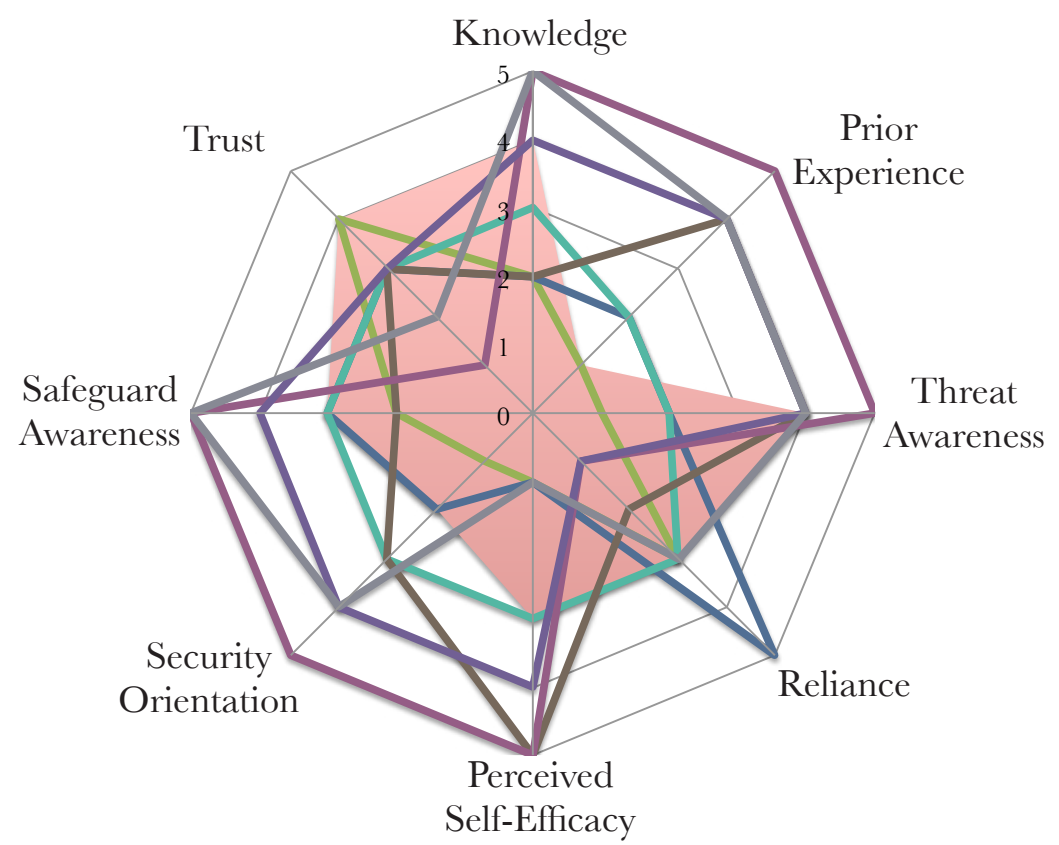

Figure 6-11. Comparison of the features of a perceived "normal user" and the identified security user types in this study

$\longrightarrow$ Normal User $\quad$ Average Respondent Based On Data

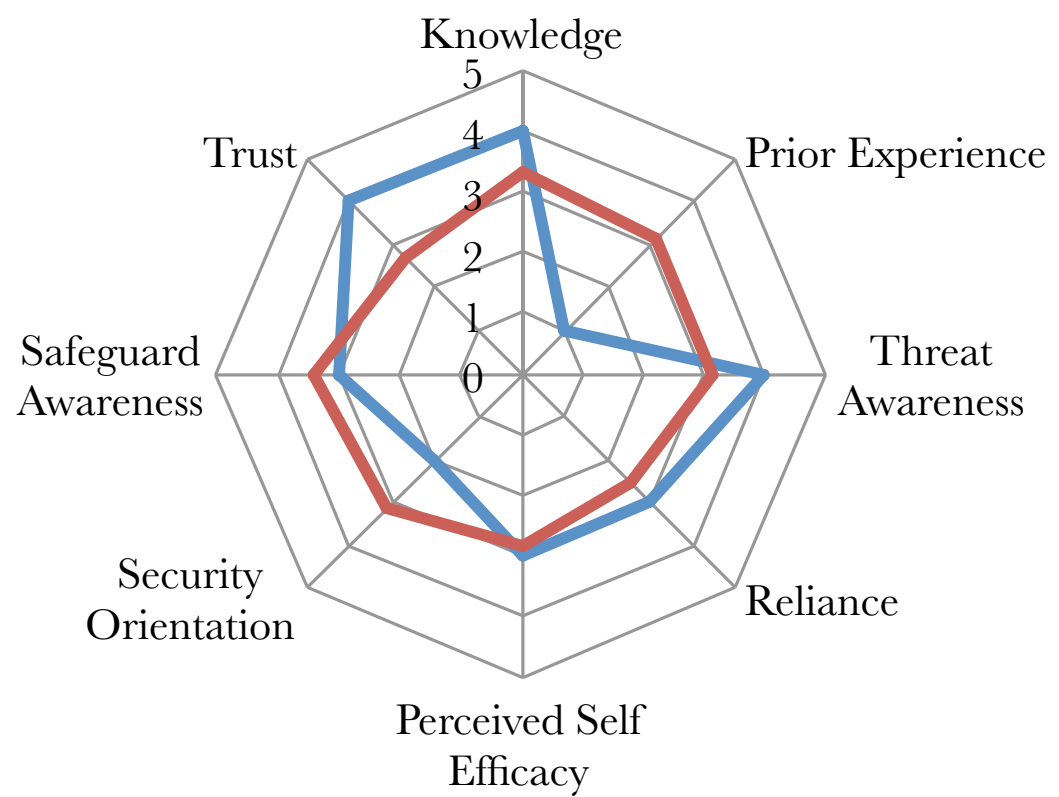

Figure 6-12. Comparison between the features of a perceived "normal user" as described by respondents and the average respondent based on the data analysis 


\subsubsection{Comparison of User Types Based on Perceived Self-Efficacy}

\begin{abstract}
Perceived self-efficacy was particularly important since individuals with higher levels of self-efficacy are more likely to try to undertake behaviours and to persevere in the case of failure (see Ajzen, 1991). Therefore, by looking at the user types based on higher and lower levels of perceived self-efficacy it was possible to see how knowledge related to the other key factors associated with the user types.
\end{abstract}

The three user types with the lowest levels of perceived self-efficacy were Ostriches, House Mice, and Dark Horses (see Figure 6-13). While House

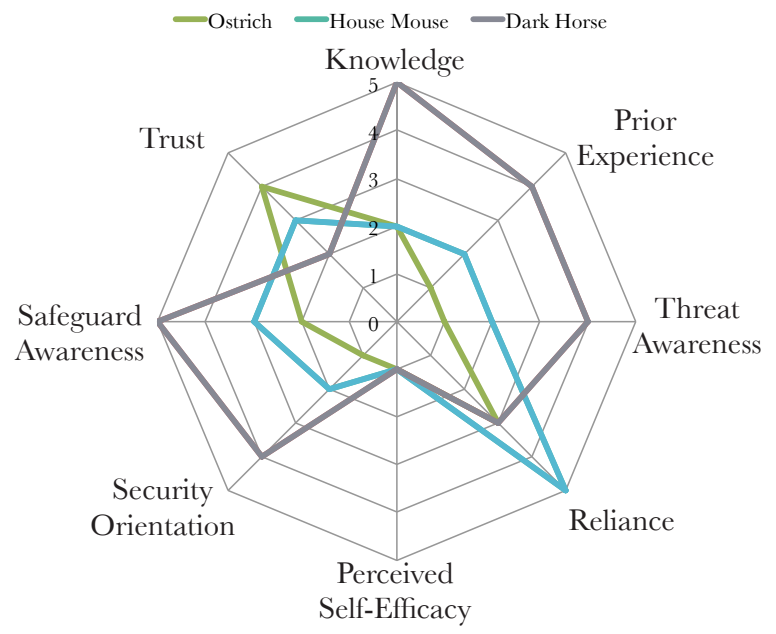

Figure 6-13. Comparison of user types with lower levels of perceived self-efficacy (Ostriches, House Mice, Dark Horses)

Mice and Ostriches shared some features, Dark Horses stood out as being particularly different. Unlike Ostriches and House Mice, Dark Horses were much more knowledgeable, aware of threats and safeguards, and were more concerned with their security. This appeared to be due to their prior experience with threats and the outcomes they experienced. Unlike the other user types with lower levels of perceived self-efficacy, Dark Horses had not only experienced breaches in the past, they had suffered what they considered to be severe consequences. This often contributed to higher levels of concern and lack of trust overall. From this, Dark Horses' confidence in their ability to protect themselves online was shaken. In contrast, Ostriches and House Mice had neither the prior experience nor the knowledge to have built up their confidence to a higher level.

Crowers, Cluckers, and Coyotes all had higher levels of perceived self-efficacy. Just as the group of users with lower perceived self-efficacy had Dark Horses that stood out as being different from the other types, within this group of users, Crowers exhibited the 


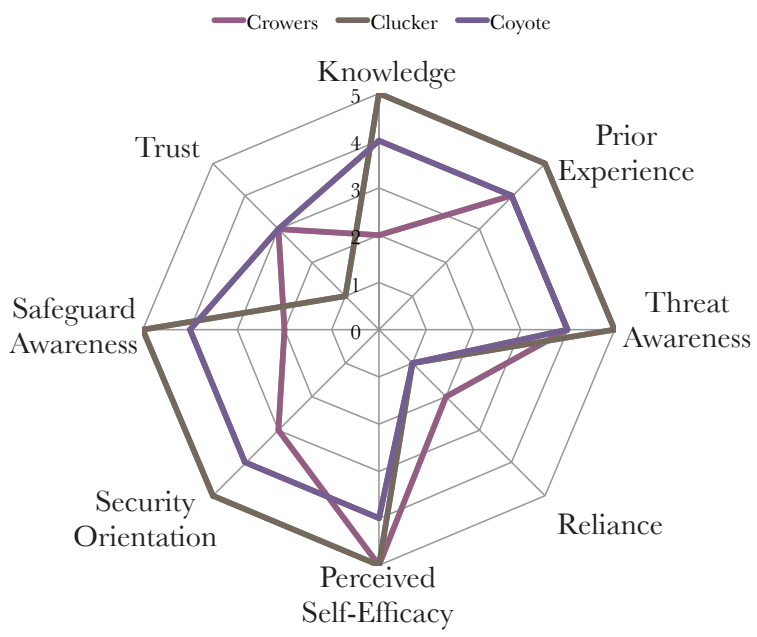

Figure 6-14. Comparison of user types with higher levels of perceived self-efficacy (Crowers, Cluckers, Coyotes) greatest difference from the other types of users (see Figure 6-14, p. 222). Unlike Cluckers and Coyotes, Crowers had much lower levels of knowledge and safeguard awareness, despite other similarities. Cluckers and Coyotes both had much higher levels of concern about security, and knowledge. Combined with their prior experiences, this led to an increased level of safeguard awareness. Both Cluckers and Coyotes were more likely than Crowers to take steps and actively seek out information about security. Crowers were much more likely than the other user types to exalt their security skills as a way to distract from their lower levels of actual knowledge. Therefore, although they had higher levels of perceived self-efficacy, overall there were enough differences that their overall behaviours were distinguishable from their counterparts with higher levels of perceived self-efficacy.

\subsubsection{Comparison of User Types based on Knowledge}

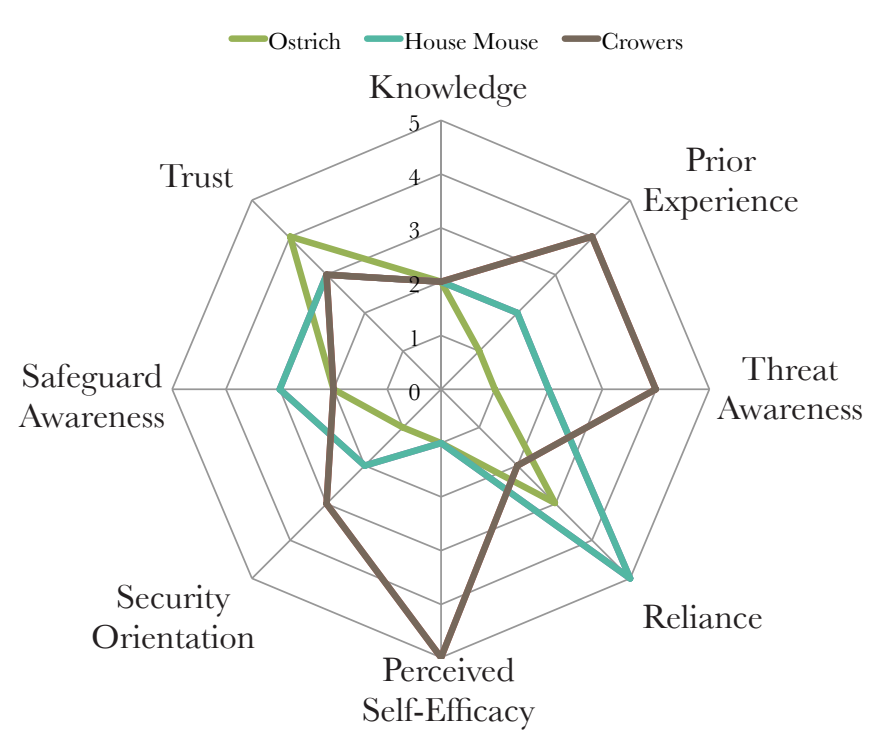

Figure 6-15. Comparison of user types with lower levels of knowledge (Ostriches, House Mice, Crowers)
Ostriches, House Mice, and Crowers all had similarly low levels of knowledge (see Figure 6-15). Once again, the Crowers stood out from the House Mice and Ostriches as being different in their overall features. The features that differentiated Crowers were those features which helped to contribute to their mien of being secure. Emphasising their prior experience, threat awareness, security orientation and perceived self-efficacy allowed them to draw attention away from their lower 
levels of knowledge. In this way, although Crowers had similar levels of knowledge to their Ostrich (see section 6.4) and House Mouse (see section 6.5) counterparts, on first impression they appeared to be much more closely related to users with higher levels of knowledge, especially if they were able to successfully distract from the lower levels of overall knowledge.

In contrast, Cluckers, Coyotes, and Dark Horses had similarly high levels of knowledge (see Figure 6-16). Unlike the group of users with lower levels of knowledge, the group with higher levels of knowledge shared more similarities in their features with similar levels of knowledge, prior experience, threat awareness, security orientation, and safeguard awareness. The group with higher levels of knowledge was much more similar than the group with higher levels of perceived self-efficacy. The main difference here was in the amount of perceived self-efficacy demonstrated by Dark Horses which was generally much lower. This seemed to indicate that knowledge, and by extension higher levels of knowledge, had a strong influence on increasing the presence of other features (e.g. awareness, prior experience, security orientation). This was because the more knowledgeable individuals were, the more likely they were to be aware of the threats and able to recognise if a security breach had taken place. Therefore, although Dark Horses had lower levels of perceived self-efficacy, their features and behaviours are much more similar to Cluckers and Coyotes.

Overall, respondents with higher levels of knowledge tended to be more secure in their behaviours, while respondents with lower levels of knowledge were less secure. Perceived self-efficacy appears to remain useful in improving users' knowledge because respondents who were more confident in their skills were more likely to increase and improve their security knowledge. However, respondents' overall level of knowledge appeared to have the greatest influence on the other factors because it led to greater awareness and recognition of threats and safeguards and greater concern about threats. 


\subsubsection{Most and Least Protective Behaviours by User Types}

The respondents whose behaviours were the most protective were the Cluckers and Dark Horses. Both Cluckers and Dark Horses tended to take the most elaborate of security steps as described by respondents. For example, such safeguarding behaviours included:

- using false names,

- disconnecting from the internet unless using it,

- using multiple forms of security software,

- investigating potential weaknesses in their systems, and

- distrusting nearly all other people online.

Both user types cited their past negative experiences with cybersecurity breaches as influencing their increased intention to engage in cybersecurity behaviours. Past experiences with breaches increased respondents' concern about security. These past events also influenced their beliefs about the likelihood of experiencing a threat since these respondents had already experienced similar events, and their beliefs about the severity of the consequences because both types of users had experienced breaches that required the respondents to invest significant amounts of time or money into repairing the situation.

The respondents with the fewest safe behaviours were Ostriches and House Mice. The most common safeguards taken by these users were:

- having antivirus software installed, and

- having someone else to take care of their security.

Ostriches tended to have unsafe behaviours because they were often unaware of the threats they faced and if they did find out about possible threats, they tended to try to avoid the entire situation. Often Ostriches simply did not know better about what they needed to do, and did not have enough concern about the danger of threats to increase their knowledge. Similarly, House Mice tended to engage in few safe behaviours on their own. Instead, they relied on others to protect their computers for them. However, the danger for House Mice 
was that at times they relied on people who were either unaware of the amount of reliance placed on them or did not have any actual responsibility for protecting the respondent's computer. Therefore, although House Mice understood more about the danger of threats and were slightly more aware of threats than Ostriches, they felt that they did not know enough to take steps to protect themselves and therefore did not take these steps themselves.

\subsection{Chapter Summary}

In this chapter, five main types of users were identified based on the factors which were the most important in influencing their cybersecurity behaviours. The users ranged from individuals who preferred to ignore threats to those who assumed that malicious users were actively trying to target them. In particular, two types of users stood out as being unique because of how they appeared in comparison to how they acted with regards to their security. Crowers appeared to be knowledgeable and safe because of their confidence in their skills. However, their actual security behaviours were not as safe as they implied because of a lack of knowledge about how to actually implement the safeguards. In contrast, Dark Horses appeared to be less safe because they were not confident in their knowledge or skills. However, their actual behaviours were amongst the safest of all the respondents. These two user types in particular illustrate the effect that perceived self-efficacy and knowledge can have in not only influencing users' actual behaviours, but also their perceptions about how safe they believe they are. In the next chapter, the value in the user types is discussed further along with why users are not as safe as they believe. 
Braun 


\section{Chapter 7
Discussion}

\subsection{Introduction}

In Chapter 4, six constructs influencing individuals' cybersecurity behaviours were identified: awareness, internal factors, external factors, perceived danger, perceived barriers and enablers, and cybersecurity intentions. Within these constructs four main sub-factors were especially influential in users' behaviours: awareness, the presence of catalyst events, knowledge, and perceived self-efficacy. In Chapter 5, a revised model was presented based on the data analysis. In Chapter 6, a set of security user types was developed in conjunction with the model from Chapter 5 which demonstrated how certain combinations of factors were related to cybersecurity behaviours. This chapter explores why awareness, catalyst events, knowledge, and perceived self-efficacy are influential in users' behaviours. Following the discussion of these key factors, the usefulness of the security user types is explored. This is followed by an examination of how the identification of these types builds on and extends some of the existing user types in the field. Finally, the reasons why individuals' perceptions about their security levels differ from what they demonstrate are explored along with why it is important to consider individuals' perceptions about security and objective measures of security. 


\subsection{Key Aspects Influencing Users' Security Intentions}

In Chapter 5, a revised model showing the factors identified in the research along with the relevant sub-factors was presented. Although there appeared to be many factors that influence individuals' security behaviours online, there were four in particular that had a greater influence because of how they related to the other constructs and factors. The initial trigger for users' security behaviours was awareness. Individuals needed to be aware of the threats before they could form opinions about the threats and the associated dangers. Closely related to awareness was whether individuals had experienced a catalyst event. Without something to trigger users' concerns they were often unaware that something could be wrong. However, to recognise catalyst events, individuals needed to have the knowledge to recognise the existence of a problem and they needed to be confident enough in their knowledge to believe that they had accurately identified a security breach. Finally, although it would seem that concern about threats would be enough to prompt individuals to engage in safer behaviours online, in many cases there was a need to deal with the selfdiagnosed problem of being "too lazy" to practise secure computing and to address the root cause of the laziness.

\subsubsection{Need for Awareness}

In the revised model in Chapter 5, awareness was the initial trigger for assessing users' intention to engage in protective behaviours online. From past literature, there appears to be an assumption that users already know what they need to protect themselves against. As a result, much of the existing literature has focused on awareness of safeguards rather than awareness of threats. For example, researchers have examined how awareness of protective technology influences the use of protective software (see Dinev \& $\mathrm{Hu}, 2007$ ) and how security awareness influences the intention to transact (see Chen \& Bansal, 2010).

Previous literature seems to assume that individuals are aware of the threats. Even if there is not an assumed level of awareness, much of the existing literature has focused on how individuals react to particular threats or their use of specific safeguards. For example, Aytes \& Connoly (2004) considered the effect of awareness of safe practices and negative consequences on password usage and email practices. They found that although users were aware of the potential negative consequences of acting in a risky way, they still continued to do so. Chen \& Bansal (2010) examined the effect of security awareness in relation to the risk of buying online. They showed a link between awareness, end-user self-efficacy, and security skill. Dinev \& Hu (2007) considered the effect of technology awareness on 
the adoption of protective software and found that attitude and subjective norm were influenced by awareness. The common thread across the previous research is that the focus is primarily on awareness of safeguards rather than awareness of threats. However, it is also useful to consider the context of the previous literature. Overall that literature has focused on awareness of very specific threats rather than more general security threats online. From this, it is possible to see that as long as the individual is aware of the threats that are being studied, for example, phishing, there is unlikely to be a need to examine broader levels of awareness and how that affects general security behaviours.

In the current research, the effect of awareness is examined more broadly. Awareness is considered within the context of both threat awareness and safeguard awareness as both are important in examining individuals' behaviours. Threat awareness is an important first step as awareness is needed for individuals to form opinions about the severity of and susceptibility to a threat (Witte, 1992 as cited in Johnston \& Warkentin, 2010). More recently, Davinson \& Sillence (2010) examined how individuals' awareness of phishing influenced their perceived susceptibility to phishing threats and found that security messages increased users' perceived susceptibility.

In addition, this research helped contribute to the field by exploring what factors contributed to users' awareness of both threats and safeguards. Although previous research has considered the effect of awareness on factors such as attitude, subjective norm, and perceived risk, there appears to be little that helps to explain what makes users aware of threats. This further supports the idea that a certain level of awareness has been assumed in the existing literature. The findings in this study suggest that beyond the media and other messages that individuals receive about security, they were also influenced by their own desire to be secure, their preexisting knowledge about security, how much they rely on others, whether they have experienced cybersecurity breaches, their trust in people and websites, and their confidence in their skills. These findings help to further understand what makes people aware of security threats and safeguards, especially in a home environment where individuals are not required to be aware of threats like they would be in an organisational environment.

Safeguard awareness is equally important for determining the behaviours individuals use to protect themselves online. Users could not be expected to engage in security behaviours they are unaware of. As indicated above, safeguard awareness has been examined in connection with specific behaviours in previous literature. In comparison, the current study positioned safeguard awareness as a prerequisite to engaging in secure behaviours. Furthermore, it examined the difference between individuals' threat awareness and safeguard awareness. In some cases there were discrepancies between threat and safeguard awareness. Most often, individuals' threat awareness was higher than their 
safeguard awareness. This may indicate that the way security information is presented is unbalanced. If individuals are presented with too much information about threats without increasing their knowledge about how to counter them, it may induce higher levels of anxiety and cause people to avoid the issue of security (for example, see Johnston \& Warkentin, 2010). This further supports the idea that in conjunction with awareness of safeguards, an element of understanding is also required.

\subsubsection{Catalyst Events}

Also important to prompting individuals to take more precautions online is the notion of a catalyst event to trigger a response (see section 4.6.1). This research examined the effect of prior experience in terms of how individuals' past experience with cybersecurity breaches affected their present security perceptions. However, the effect of prior experience is closely related to whether an individual has experienced a catalyst event. Although one may expect that the absence of a catalyst event would mean that an individual cannot form perceptions in relation to their security, the lack of a catalyst event too affects individuals' perceptions about security and safeguards.

Individuals who had not experienced a cybersecurity breach or another catalyst event often did not have the necessary frame of reference against which to judge the possible danger of online threats. In addition, at times respondents were more likely to assume that they were safe simply because of the lack of experience with a catalyst event. Rhee et al. (2005) suggested that users often exhibited an optimistic bias when assessing their security, arguing that "the unrealistic optimism observed in the domain of information security may lead people to ignore the measures and practices to offset information security threats" (p. 12). Similarities were seen in the current study in the way that respondents considered themselves to be safer because they did not fit one of what they considered to be the main criteria for a person to be the target of a malicious user. Therefore it appears that individuals who have not experienced a cybersecurity breach often consider the threat to be too far removed from their personal circumstances to consider it a true danger.

Good et al. (2006) noted that users who had negative experiences online were more cautious about reading EULAs (end user licensing agreements) and installing software found on the internet. However, the existence of a catalyst event does not necessarily trigger more concern about security. Instead, how an individual deals with the event and the type of consequences they experience is more likely to influence their concern about security. As indicated in section 4.5.1 there were two main ways that prior experience influenced respondents' concern about security overall. First, individuals who had negative experiences, such as data loss where it was particularly difficult to return to the point before 
the loss, were often more concerned about security overall. In contrast, there were other respondents who had experienced catalyst events, but the way that they had been able to cope with them and the ease with which they had returned to the status quo meant that they were less concerned. By easily overcoming the potential issue and minimising the perceived severity of the breach, these users developed the confidence to cope with the breaches, which in turn made them less concerned about the overall danger. This indicates that while the presence of a catalyst event can improve individual concern, the mere presence of such an event does not guarantee an increased concern. Instead, it is important to look at how the individual has reacted to the event and the outcomes.

Despite the different possible ways that previous experience can influence individuals' concern about security, it remains that individuals who have experienced catalyst events are more concerned about security than those without the experience. However, this also raises several difficulties when it comes to increasing users' concerns about security and in turn improving their security behaviours. Often individuals assume that they will recognise the symptoms of a breach and that every breach will display tangible symptoms. In 2010 an estimated 35 percent of computers were connected to at least one botnet (Wilson, 2011). However, in many cases users were "oblivious to the infection" (Webb, 2007). If individuals cannot recognise that an event has occurred, it is likely to have the same effect as if nothing had happened because there is nothing to trigger the behavioural change. There are two main issues which come out of the assumption that a breach will be recognisable enough to be considered a catalyst event. First, users have to be able to recognise the symptoms of a breach. Second, they have to understand what the potential consequences are. Both of these require a certain level of knowledge.

Furthermore, it appears that in many cases, users depend on the presence of a catalyst event to trigger a change in a their behaviours. Without some form of catalyst event, there is a danger that people will continue to consider themselves to be invulnerable to threats (see Johnston \& Warkentin, 2010). This suggests that security is often viewed by users as a reactive process rather than a proactive one. The idea that users depend on the presence of a catalyst event raises further issues because it is unrealistic to assume that all users will have experienced a security breach. However, this again returns to the issues raised earlier. Not only do people need to recognise that a breach has occurred, they also need to understand the particular consequences and consider them severe enough to elevate the trigger to the level of a catalyst event. As noted above, there is still the issue of how the individual will react to the breach which in turn influences how they perceive the overall danger. Therefore, while a catalyst event is an important part of determining how concerned an individual will be about the danger, alone it is unlikely to provide the sole impetus for change. 


\subsubsection{Knowledge and Perceived Self-Efficacy}

Perceived self-efficacy refers to a person's "judgements of how well one can execute courses of action required to deal with prospective situations" (Bandura, 1982, p. 122). In contrast, knowledge refers to the actual understanding that individuals have about cybersecurity, regardless of whether they act on that knowledge. Knowledge and selfefficacy have been considered together with awareness by Chen and Bansal (2010) in their study on perceived security risk. It is important to note that while both perceived selfefficacy and knowledge are important factors influencing users' cybersecurity behaviours, the presence of knowledge and skill is often not enough to assume that individuals will take the appropriate steps to protect themselves (see Bandura, 1982). Because of this, knowledge and perceived self-efficacy are among the main factors that influence users' behaviours.

As noted in the above section, without the knowledge of what the symptoms of a security breach are, users may be unable to recognise one. Therefore, there is a certain level of understanding required by the individual in order for a catalyst event to have an effect. For example, if an individual is unaware of the consequences of malware, they may not understand that their computer has been compromised. Similarly, even if a person does recognise that their computer has been compromised, if they are unsure of what the consequences are they are less likely to be motivated to improve their behaviours as they see little effect on their daily lives. The result is that without a base level of knowledge, users are unlikely to consider the threats to be particularly dangerous. In turn, this contributes to users further dissociating themselves from the threat overall, and minimising the effect of previous experience on users' intention to protect themselves.

Furthermore, if users do not understand the threat or its associated consequences, there is a risk that they will consider themselves to be safer than they should. For example, respondents who felt that they had come through a security breach without suffering any consequences felt more secure online and had higher levels of perceived self-efficacy because they felt that they could remedy a breach if one were to occur. However, high levels of perceived self-efficacy combined with lower levels of actual knowledge contribute to users being more likely to have a false sense of security and in turn engage in more risky behaviours out of naïveté.

In order to create safer users, a combination of knowledge and perceived self-efficacy is needed. Respondents who had higher levels of perceived self-efficacy in many cases were more likely to read and seek out information about security which increased their knowledge. In contrast, individuals with lower levels of perceived self-efficacy were less likely to seek out the information since they did not know where to start looking or how to judge whether the information was relevant. As a result, respondents with lower levels 
of perceived self-efficacy tended to have lower levels of knowledge. Similarly, respondents with higher levels of knowledge were often more likely to seek out additional information to increase their knowledge further since they believed that they had the necessary skills to find and make use of the information.

Perceived self-efficacy has been shown to have a positive influence on intention to engage in protective behaviours (see Chan et al., 2005; Johnston \& Warkentin, 2010; Liang \& Xue, 2010; Ng et al., 2008; Pahnila et al., 2007). Perceived self-efficacy has also been shown to have a positive influence on perceived behavioural control (see $\mathrm{Ng} \&$ Rahim, 2005). While Dinev and $\mathrm{Hu}$ (2007) found a the relationship between self-efficacy and perceived behavioural control to be statistically nonsignificant, they indicated that this was likely due to the specificity of the technology they were examining (anti-spyware software). While perceived self-efficacy has been well established as an important factor influencing users' behaviours, there has been a gap in understanding what generates these feelings of self-efficacy in relation to users' security behaviours. The literature shows that high levels of self-efficacy are an important part of increasing users' intention to protect themselves online. However, without understanding what factors help to generate these high levels of self-efficacy it is difficult to understand what steps can be taken to improve users' beliefs that they can protect themselves online. Therefore, by exploring what factors help increase users' perceived self-efficacy it may be possible to increase the likelihood that individuals will believe that the steps they take to protect themselves can make a difference.

On a more practical level improving users' knowledge through security information campaigns is not necessarily enough to improve users intention to engage in protective behaviours. Instead, there needs to be a focus on improving users' perceived self-efficacy in conjunction with improving their knowledge. The impact of lower levels of perceived self-efficacy is seen with respondents who also have lower levels of safeguard awareness. This suggests that there is a link between improving users' perceived self-efficacy and safeguard awareness. By focusing not just on informing users of what the risks are and instead focusing on making sure that users' know what steps they can take as well as the effectiveness of those steps, it may help improve users' security overall as it can help to minimise the fatalistic attitude that many respondents displayed toward the benefits of security behaviours.

\subsection{Security User Types}

As noted in Chapter 6, it was possible to categorise users into different types based on the factors identified in the model presented in Chapter 5. The revised model (Figure $5-2$, p. 141) illustrates how the factors relate to each other and how these factors can 
lead users to make decisions about their security. The user types identified in Chapter 6 serve to further illustrate how the internal and external factors work together and help influence users' behaviours. The development of the user types has two main benefits: first, it illustrates the revised model presented in Chapter 5; second, it provides a starting point for examining how users' security behaviours can be improved by addressing behaviours on a more individual level rather than assuming that security messages should take a onesize-fits-all approach.

\subsubsection{Comparison to Existing User Types}

In the past, researchers have categorised users into different types based on characteristics and behaviours. Within the security context, the types identified have predominantly been associated with privacy concerns (see Table 7-1, p. 235). For example, Sheehan (2002) examined users' behaviours related to their responses to spam, based on their privacy concerns. In particular, Sheehan's research found that age and education played the strongest role in determining users' behaviours. Drennan, Mort and Privite (2006) also examined privacy, instead focusing on awareness of privacy policies and knowledge of the potential issues associated with privacy. Barnes, Bauer, Neumann, and Huber (2007) identified clusters of users based on their beliefs about online shopping, in particular, looking at the effect of perceived risk of shopping online and trust. Finally, Tshohou, Karyda, Kokolakis, and Klountouzis (2006) used established cultural biases in their examination of risk management to determine how these biases not only affected IS risk management, but also how the knowledge about these biases could be utilised to develop more effective risk management strategies.

This literature had strong focus on privacy when creating user types. In Chapter 2, the relationship between privacy and security was discussed. By engaging in security behaviours, users can also protect their privacy. Yet focusing on privacy does not always take into consideration the security issues that may arise online (e.g. that do not infringe on individuals' privacy). Therefore, while the user types that focus on privacy are useful, they do not necessarily provide sufficient explanation for security behaviours on their own.

In addition to privacy, previous research has also focused on categorising users by how concerned they are about a risk, such as online privacy risks (for example, see Drennan et al., 2006; Sheehan, 2002). The user types in the current research go beyond the level of concern about security. Concern about security, primarily discussed in section 4.4 and section 4.5.6, is only one part of answering why users take the steps that they do in order to protect themselves online. Within the user types, examining individuals' security orientation plays a larger role than perceived danger since it does not require an external event, such 
Table 7-1. Summary of user types and clusters related to internet security and privacy

\begin{tabular}{|c|c|c|}
\hline Author(s) & $\begin{array}{l}\text { Summary of } \\
\text { Research }\end{array}$ & Overvieze of User Types \\
\hline Sheehan (2002) & $\begin{array}{l}\text { Individual } \\
\text { concern about } \\
\text { online privacy in } \\
\text { regards to spam. }\end{array}$ & $\begin{array}{l}\text { Found the age and level of education influenced } \\
\text { users' behaviours and identified four types of } \\
\text { individuals based on these factors and users' } \\
\text { actual behaviours. The types of users were (1) } \\
\text { unconcerned internet users, (2) circumspect } \\
\text { internet users, (3) wary internet users, and (4) } \\
\text { alarmed internet users. }\end{array}$ \\
\hline $\begin{array}{l}\text { Rifon, Quilliam \& } \\
\text { LaRose (2005) }\end{array}$ & $\begin{array}{l}\text { Individual } \\
\text { motivations } \\
\text { for engaging } \\
\text { in protective } \\
\text { behaviours. }\end{array}$ & $\begin{array}{l}\text { Utilised categories of internet users based on } \\
\text { their experience and safety involvement in order } \\
\text { to select focus group participants. The categories } \\
\text { used were: (1) newbie, (2) brave, (3) confident, } \\
\text { and (4) reckless. }\end{array}$ \\
\hline $\begin{array}{l}\text { Tshohou, Karyda, } \\
\text { Kolkolakis \& } \\
\text { Klountouzis (2006) }\end{array}$ & $\begin{array}{l}\text { Information } \\
\text { system risk } \\
\text { management } \\
\text { strategies based } \\
\text { on cultural } \\
\text { theories. } \\
\end{array}$ & $\begin{array}{l}\text { Applied the four types of cultural bias (fatalism, } \\
\text { hierarchy, individualism, and egalitarianism) to } \\
\text { the issue of information system risk and discussed } \\
\text { how these topologies could be applied in the } \\
\text { initiation, risk analysis, and risk mitigation stages } \\
\text { of risk management planning. }\end{array}$ \\
\hline $\begin{array}{c}\text { Drennan, Mort \& } \\
\text { Previte (2006) }\end{array}$ & $\begin{array}{l}\text { Privacy concerns } \\
\text { regarding privacy } \\
\text { policies and } \\
\text { information } \\
\text { sharing by } \\
\text { businesses. }\end{array}$ & $\begin{array}{l}\text { Examined individuals' awareness and knowledge } \\
\text { of privacy to identify types of privacy statements. } \\
\text { Identified three types of statements about privacy } \\
\text { by individuals: (1) privacy aware, (2) privacy } \\
\text { active, and (3) privacy suspicious. }\end{array}$ \\
\hline $\begin{array}{c}\text { Barnes, Bauer, } \\
\text { Neuman, \& Huber } \\
\text { (2007) }\end{array}$ & $\begin{array}{l}\text { Development } \\
\text { of a customer } \\
\text { typology } \\
\text { regarding } \\
\text { online shopping } \\
\text { intentions. }\end{array}$ & $\begin{array}{l}\text { Identified and grouped individuals based on } \\
\text { their neuroticism, extraversion, trust, attitude } \\
\text { toward online shopping, perceived risk, shopping } \\
\text { enjoyment, and willingness to buy. Three clusters } \\
\text { of customers were identified: (1) risk adverse } \\
\text { doubters, (2) open-minded online shoppers, and } \\
\text { (3) reserved information seekers. }\end{array}$ \\
\hline
\end{tabular}

as a specific threat, in order to be present. However, by examining security orientation within the user types, it is still possible to look at the effect of concern as part of the larger concept of how certain factors unique to users can interact to influence how they approach security. This moves the value of the user types beyond concern about security, instead taking a broader approach in understanding why concern on its own is not enough to prompt individuals to protect themselves online.

The main difference between the previously identified user types and the user types identified in the current research lie in the factors used to identify the types. As noted, there appears to be little research done on how threat awareness influences security behaviours. 
Although Drennan et al. (2006) examined awareness, it was done in regard to awareness of privacy policies.

Finally, it is useful to look at users' security behaviours in terms of user types as it has been noted that personalised language in security messages has the ability to improve individuals' perceptions of susceptibility to threats (for example, see $\mathrm{Ng}$ et al., 2008; Witte \& Allen, 2000). Although susceptibility alone does not drive users to protect themselves from threats online, it stands to reason that by examining how other characteristics drive users' behaviours, we can work towards creating more effective security messages. This applies not only to messages about what the threats are and why users need to be concerned about them, but also to how users can protect themselves from these threats.

From the current research and past research, it appears that while awareness of threats is a necessary starting point for looking at cybersecurity behaviours, what becomes more important is that individuals know how to protect themselves and have the confidence to do so. Without this confidence, as discussed in section 4.5.3, individuals are much less likely to take the steps needed to protect themselves online, often preferring to either ignore the threat or pass the responsibility for protection on to someone else.

This in turn further emphasises the need to make cybersecurity more relatable to individuals. However, in order to make the security messages more personal, there is a need to understand how individuals approach security. The security types developed in Chapter 6 are a step towards being able to do this. By focusing on the interaction between different factors we can see which types of users need to improve their confidence (i.e. Ostriches, House and Field Mice, and Dark Horses), their knowledge (i.e. Ostriches, House Mice, and Crowers), and their use of protective behaviours (i.e. Cluckers and Coyotes). By personalising security messages that address these main issues for each of the user types, it is possible to benefit from the strengths of each of the types of users, while focusing on areas that may be weaker. It also helps to increase the perception of danger that individuals may lack due to the combination of the above mentioned factors.

Respondents in the study occasionally indicated that security information was irrelevant to them, for example by noting that they did not need to be as concerned because they were not a business. In addition, there were often implications that security was not relevant either because someone else took care of it or because there was too much information to sort through in order to determine what information was relevant. This further emphasises the need for individuals to be presented with information that they consider not only relevant, but also useful. This sort of message is unlikely to be presented in a one-size-fits-all approach to security as it assumes that users either have very little knowledge and need everything done for them or that the user has sufficient knowledge to successfully navigate potentially complicated software. The result seems to 
be that individuals consider security to be a one-step solution where they install a program and forget about it until they need to renew a license. Therefore, there is a need to consider how security can be made more relevant to individual users. One way this can be done is by addressing the security behaviours by user types.

\subsubsection{Recommendations for Safer Behaviours by User Type}

Although at the outset of the research identifying user types was not one of the original objectives, the existence of possible user types emerged from the collected data. The user types offered a way to apply the main factors that influenced users' cybersecurity intentions and illustrated how various factors interacted with each other. Five main types of users were identified: Ostriches, Mice, Cockerels, Coyotes, and Dark Horses. Within these user types, Mice were further divided into House Mice and Field Mice, while Cockerels were divided into Crowers and Cluckers. These user types each present their own challenges which need to be overcome to increase individuals' intentions to engage in protective behaviours. A summary of the main challenges for users and an overview of recommendations is found in Table 7-2, p. 238. These recommendations are further explained below and focus on ways that security and security messages can be targeted at the user types since it has been shown that targeted security messages are more effective at improving users' behaviours (for example, $\mathrm{Ng}$ et al., 2008; Witte \& Allen, 2000).

Ostriches are characterised by their desire to hide from security threats in order to feel safer about not taking steps to protect themselves. The main features that distinguish $O$ striches are their low levels of knowledge, awareness, perceived self-efficacy, and prior experience, along with moderate to high levels of reliance. The main challenge for addressing Ostriches' cybersecurity behaviours is convincing them to protect themselves since they typically have not experienced cybersecurity breaches in the past, which has led them to believe that it is acceptable to ignore threats online. As a result, to increase Ostriches' intentions to engage in more cybersecurity behaviours, there needs to be a focus on increasing their knowledge, awareness, and perceived self-efficacy. In addition, attention needs to be paid to ensuring that Ostriches feel that security is relevant. If Ostriches do not feel that security is relevant, they are unlikely to take steps beyond those they are already taking since so far, these steps have yielded sufficient protection. However, because Ostriches often lack knowledge and awareness, they may be mistaken in their beliefs that they are secure. Because of this, it is even more important to ensure that security messages are approached in a way that provides Ostriches with a reason to stop ignoring potential threats and to take steps to increase their protective behaviours. 
Table 7-2. Summary of challenges and recommendations by user type.

\begin{tabular}{|c|c|c|}
\hline User Type & Main Challenges & Recommendation(s) \\
\hline Ostrich & $\begin{array}{l}\text { Low awareness and security } \\
\text { orientation lead to ignoring risks. }\end{array}$ & $\begin{array}{l}\text { - Increase knowledge, awareness, } \\
\text { and security orientation. } \\
\text { - Focus on making security } \\
\text { relevant. }\end{array}$ \\
\hline House Mouse & $\begin{array}{l}\text { High reliance on others leads } \\
\text { to lowered sense of personal } \\
\text { responsibility. }\end{array}$ & $\begin{array}{l}\text { - Increase knowledge and } \\
\text { perceived self-efficacy to help } \\
\text { decrease reliance. } \\
\text { - Make security relevant. }\end{array}$ \\
\hline Field Mouse & $\begin{array}{l}\text { Enough knowledge to take basic } \\
\text { protective measures, but not } \\
\text { enough concern to keep up with } \\
\text { changing threat landscape. }\end{array}$ & $\begin{array}{l}\text { - Increase awareness and } \\
\text { knowledge of threats to promote } \\
\text { updated security knowledge. }\end{array}$ \\
\hline Crozerer Cockerel & $\begin{array}{l}\text { High levels of perceived self- } \\
\text { efficacy are not supported by } \\
\text { lower levels of knowledge, leading } \\
\text { to distorted views of how secure } \\
\text { these users are. }\end{array}$ & $\begin{array}{l}\text { - Focus on improving knowledge } \\
\text { and awareness through their } \\
\text { high levels of perceived self- } \\
\text { efficacy. } \\
\text { - Promote how security is } \\
\text { relevant. } \\
\end{array}$ \\
\hline Clucker Cockerel & $\begin{array}{l}\text { Very low trust and high security } \\
\text { orientation leading to "paranoia." }\end{array}$ & $\begin{array}{l}\text { Focus on increasing knowledge } \\
\text { and awareness of how their } \\
\text { extensive security keeps them } \\
\text { safe and why it is safe to do } \\
\text { more online. }\end{array}$ \\
\hline Coyote & $\begin{array}{l}\text { Confidence in skills with a } \\
\text { willingness to take risks. }\end{array}$ & $\begin{array}{l}\text { - Focus on making security } \\
\text { relevant while promoting how } \\
\text { users can still stay safe while } \\
\text { engaging in the behaviours that } \\
\text { may be increasing their level of } \\
\text { risk. }\end{array}$ \\
\hline Dark Horse & $\begin{array}{l}\text { Low perceived self-efficacy leads } \\
\text { to down-playing skills and a lack } \\
\text { of confidence in their ability to } \\
\text { protect themselves. }\end{array}$ & $\begin{array}{l}\text { - Focus on increasing perceived } \\
\text { self-efficacy to increase } \\
\text { confidence in the skills the users } \\
\text { already have. } \\
\text { - Care needs to be taken to avoid } \\
\text { increasing concern to level of } \\
\text { "paranoia." }\end{array}$ \\
\hline
\end{tabular}

Mice are closest to what can be considered the average users, and were characterised by their generally timid nature. However, House Mice and Field Mice need to be approached differently when trying to increase their intention to engage in protective behaviours. House Mice are distinguished by their very low levels of perceived self-efficacy and very high levels of reliance on others. The result is that House Mice tend to take very little responsibility for their own actions and instead push the responsibility on to someone else who may not be aware of the responsibility that they have been assigned. Although 
House Mice may be protected online, it is often not due to any steps that they have taken themselves. Therefore, the challenge with House Mice is to find a way to increase their personal sense of responsibility for being secure online in order to avoid naïve mistakes. The combination of low levels of perceived self-efficacy and high levels of reliance can be particularly problematic to address if House Mice feel that they are being adequately protected, since this can make it more difficult to convince them that there is a need to act in a secure manner. It may be possible that increasing their knowledge, awareness, and perceived self-efficacy could help to alleviate some of the dependance on others. Like Ostriches, there is a similar need to ensure that security messages are targeted in a way that increases the House Mouse's belief that the threat is relevant.

Although Field Mice tend to take more steps to protect themselves online and rely less on others than their House Mice counterparts, there are still ways that their behaviours can be improved. Field Mice are the normal internet user. They have moderate levels of almost all of the relevant factors. The biggest challenge with Field Mice is convincing them to take more steps to protect themselves and to become more familiar with the changing threat landscape. One of the ways that this could be done is to focus on increasing their awareness and knowledge of threats. One of the benefits of Field Mice is that they are already aware of the need for security, which increases their likelihood of undertaking protective behaviours. Therefore, if their knowledge can be modernised to help address the new ways that threats are being distributed, they would be likely to be even safer online. In addition, increasing this awareness and confidence may further improve their perceived self-efficacy.

Cockerels are characterised by their high levels of perceived self-efficacy and have the highest level of all the identified user types. Crowers are further characterised by their lower levels of knowledge and lower levels of safeguard awareness. The biggest challenge with Crowers is in getting them to recognise that they are overconfident in their ability to protect themselves. Because of their high levels of perceived self-efficacy, they may not recognise that their knowledge and awareness is lacking. This can lead to boasting about their security prowess, even though they tend to take few protective measures. As a result, Crowers may not consider the threats to be relevant to them because they believe that they are protected, which leads to a decreased sense of danger. Therefore, the first step may be to present security messages in a way that makes them relevant to Crowers. The second thing that needs to be done to increase Crowers intention to engage in security behaviours is to improve their knowledge and awareness. The high levels of perceived self-efficacy may be beneficial if the Crowers believe that they can actually follow through on steps to protect themselves once presented with the relevant information. However, the danger is that the perceived self-efficacy may be more of a façade and therefore, Crowers may require a similar approach to Ostriches and Mice to help increase their intention to engage 
in protective behaviours.

Cluckers are very secure in their behaviours and can be identified by their very high levels of threat and safeguard awareness, knowledge, perceived self-efficacy, prior experience, and security orientation, along with very low levels of trust and reliance. Unlike Crowers, Cluckers' confidence in their skills is justified. Cluckers take numerous steps to protect themselves. However, these extensive steps can result in a situation where the Clucker is effectively unable to use their computer out of concern that something could go wrong. As a result, the biggest challenge with Cluckers is necessarily improving their behaviours, but instead focusing on increasing their awareness of how they can make better use of their computer while remaining safe. This can be particularly challenging though as Cluckers often self-identified as being "paranoid" about their security. Therefore, the focus needs to be on increasing knowledge and awareness in the practical sense of ways that their security needs can be met while increasing the number of tasks that they are comfortable doing on their computers.

Coyotes are the most likely type of users to knowingly take risks online because of their confidence in their ability to remedy the consequences of any breaches that may occur. Coyotes can be identified by their moderate to high levels of all of the identified factors except for moderate levels of trust and low levels of dependence. Coyotes often have experienced cybersecurity breaches and have resolved the consequences of them themselves. The result of this is a high level of confidence that if something goes wrong, they believe they can fix it. This makes Coyotes more likely to take risks if they know that the outcome is likely to be beneficial to them. As a result, the challenge is to decrease their risktaking behaviours. The biggest difficulty in doing this is that their abilities have frequently been reenforced. Therefore, part of the solution may be to focus on making security more relevant to Coyotes and presenting security messages in a way that emphasises the ongoing or more longer term consequences that can be associated with breaches in a way that complements their understanding of how to remedy short term breaches.

Finally, Dark Horses are characterised by moderate to high levels of threat and safeguard awareness, knowledge, security orientation, and prior experience, combined with low levels of perceived self-efficacy, trust, and moderate levels of reliance. Dark Horses can be considered the opposite of Crowers. Where Crowers have high levels of perceived self-efficacy and low levels of knowledge, Dark Horses have low levels of perceived self-efficacy and high levels of knowledge. Therefore, the greatest challenge with Dark Horses is to increase their confidence in their skills. The main benefit of increasing their confidence is that they may be more likely to help increase the knowledge of other users, particularly if they share their computer. Without this confidence, Dark Horses are still likely to take numerous steps to secure their computers. However, no matter how many steps they take, they still feel 
unprotected. The danger in these feelings is that they can rise to the level of "paranoia" about security at times. Therefore, an increase in perceived self-efficacy may help Dark Horses to understand that they may be protecting themselves better than they realise.

Overall, in many cases it appears that users feel that the threat is not relevant, usually because they do not feel susceptible or they do not think the consequences would be severe. As a result, many of the user types would benefit from security messages that focus on making the need for security more relevant to the individual users, rather than security being presented in very broad terms without clear benefits. Some user types would also benefit from increased knowledge. However, without making security relevant, it is unlikely that individuals would see the benefits of increasing their knowledge.

\subsection{Are People as Secure as They Think?}

From the literature, it was expected that there would be a difference between how respondents saw themselves and what the reality was (for example, see Rhee et al., 2005; Woon et al., 2005). The term optimistic bias has been used to describe how "people interpret ambiguous information or uncertain situations in a self-serving direction" (Rhee et al., 2005, p. 4). In the context of internet security, this would suggest that people believe they are safer than the average person from threats online. However, from the research it seems that although some respondents considered themselves to be more secure than the normal user, more considered themselves to be less knowledgeable, aware, and secure than the normal user, demonstrating more of a pessimistic bias. A pessimistic bias relates to how "egocentric processing may lead people to believe that they are uniquely vulnerable to the effects of severe life events when these events remain in the abstract" (Blanton, Axsom, McClive, \& Price, 2001, p. 1629). By looking at the factors which may lead to these biases, it helps to show where people are both more and less confident in their abilities, and whether they really are as secure as they believe themselves to be.

As indicated in section 6.3, respondents were asked to describe the normal user, which created a portrait of how respondents viewed them. Respondents often compared themselves to the normal user, so it was important to understand what they meant. The features of the perceived normal user can be compared then to the average respondent based on the data (see Figure 7-1, p. 242). There are five main factors where respondents' perceptions of the average user differed from the average respondent in this study. Respondents were more likely than their perceived counterparts to have experienced a breach in the past. Respondents were also more likely to be concerned about their security than they believed the average user to be. In contrast, respondents were less aware of threats than they believed the average user to be. Based on the data, the average respondent was also less 


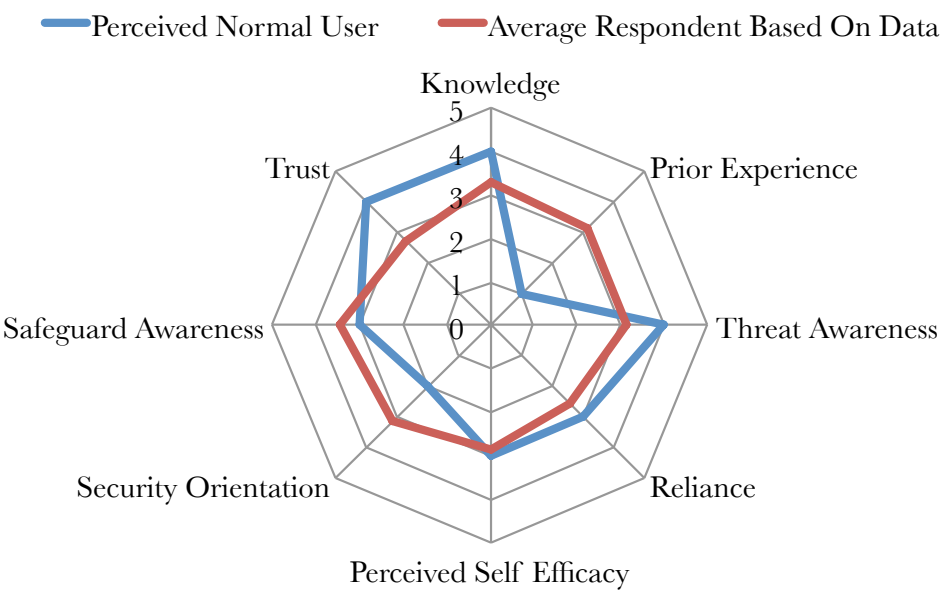

Figure 7-1. Comparison between the features of a "normal user" and the average respondent based on the data analysis

knowledgeable and less trusting than they believed the average user was.

Knowledge, security orientation and threat awareness had clear distinctions between the perceived normal user and the average respondent. Respondents who had higher levels of these factors were more likely to engage in safer behaviours. However, overall, respondents demonstrated a more pessimistic assessment of these factors when they compared themselves to the average user. This goes back to the idea that most people considered the basics of security to be ingrained in the culture (see section 4.3). Therefore, if they felt that they did not know much about security, there was a tendency to believe that the average user knew more. However in terms of the idea that security is ingrained in the culture, this also caused a belief that because it was so ingrained, people did not need to worry about it. Yet respondents often worried about it more, often because they did not feel they would have the knowledge or awareness required to protect themselves.

\subsection{Why Are People Lazy About Security?}

A final point of interest is the idea that most users consider themselves to be "lazy" about security. It was expected that some people would simply be unconcerned about their security. However, it was unexpected that so many people would blame laziness for their failure (both real and perceived) to take more precautions. As indicated in section 4.5.7, almost every respondent indicated that laziness was an issue when deciding what level of protection to undertake online.

Laziness was a concept that first emerged during the focus group discussions when respondents were asked to identify reasons why they did not take as many precautions as they thought they should. At first, it seemed likely that laziness would be limited to focus group participants since it could provide them with a way to avoid seeming to have either 
too much or too little knowledge. The excuse of laziness could have been used to allow participants to conform to what they felt others would expect of them. However, the notion of laziness was not confined to the focus groups. The trend toward blaming laziness continued in the interviews.

Individuals were often open about not considering themselves to be aware of threats (see section 4.3) or knowledgeable about them (see section 4.5.2). Past security research has shown that although individuals know what they should do to protect themselves, they still report acting in less secure ways (see Davinson \& Sillence, 2010). Overall, individuals appeared to prefer to consider themselves lazy about security rather than being unknowledgeable or unconcerned.

Laziness often appeared to be used as a way for individuals to distance themselves from the perceived danger of security threats online. However, it also appeared to be used as a catch-all excuse to encompass reasons why individuals did not do more to protect themselves. In order to explore why laziness was so commonly mentioned in the study, it is useful to be clear on whether laziness had the same meaning for everyone.

Some respondents preferred to be considered lazy rather than being seen as lacking awareness or understanding of the threats and safeguards. In these cases, laziness was used as an explanation for minimal security behaviours in place of a lack of knowledge. How the term "laziness" was used depended on how much knowledge respondents believed they had compared to how much knowledge they demonstrated. Where respondents had indicated lower levels of knowledge, laziness was often used to describe why they did not take steps to improve their knowledge. In this way laziness related back to their information seeking behaviours which in turn were influenced by their understanding of security risks.

In contrast, where there were individuals who considered themselves to be knowledgeable but did not demonstrate this knowledge, the excuse of laziness was a way to keep attention away from their lower levels of knowledge. In these cases, the respondents preferred to be considered lazy rather than unknowledgeable. Using the excuse of laziness allowed respondents to focus more on their concern about the threats rather than their knowledge of them. These individuals often distanced themselves from the concern over the potential dangers as a way to justify what they described as their laziness.

As indicated in section 4.4 respondents stated that they did not need to be concerned about security because they were not the intended targets of malicious users. They believed they would not be targeted because they did not consider themselves to have anything of value on their computers. Therefore, they considered it to be acceptable to be lazy about security because they did not see a reason to be concerned. However, these respondents often considered themselves to be knowledgeable about security but did not demonstrate the high levels of knowledge they professed. When the discussion then turned to what 
these individuals did to protect themselves online, their protective behaviours were often minimal. Laziness as a justification for not engaging in more protective behaviours then indicates that these individuals were likely to be aware that they could be doing more to protect themselves online. However, in combination with their lower levels of knowledge, they needed to find a way to justify essentially knowing better while not doing better. In this case, laziness then became the justification for their behaviours based on their overall lack of concern about being targeted.

Furthermore, the majority of respondents indicated that internet security knowledge and behaviours were ingrained in everyone's culture. Based on these beliefs, there seemed to be agreement that people should be so familiar with security that they have no need to be concerned, nor do they have any need to seek out additional information. However, this returns to the idea of individuals distancing themselves from internet security either because they do not know where to start or because they are not concerned about it.

Psychology research suggests that individuals who want to be considered to be more sociable tend to downplay their knowledge (for example, see Holoien \& Fiske, 2012; Kervyn, Judd, \& Yzerbyt, 2009). Individuals who are seen as being "warm" ${ }^{1}$ are perceived as being warmer when they are less competent (Kervyn et al., 2009). Similarly, being naive is considered to be better intellectually than being unintelligent (Fiske, Cuddy, \& Glick, 2007). Both Holoien \& Fiske (2012) and Kervyn et al. (2009) consider laziness to be ranked in a similar way to naïveté and a lack of intelligence in terms of competence and warmth. This may support the idea that respondents in the current study considered laziness to be more acceptable than a lack of knowledge. With regard to respondents who wanted to present a mien of being knowledgeable and cautious yet considered themselves to be lazy about security, the explanation may be less straight forward. Again, while presenting a facade of competence, their actual knowledge was much lower. This suggests that the excuse of laziness may be more closely related to individuals' actual levels of knowledge, which in turn drives them to be considered to be warmer through lower levels of competence. Therefore, even when individuals present themselves as lazy, it may not be actual laziness that is driving their security behaviours, but rather their knowledge.

\subsection{Chapter Summary}

In this chapter the key factors relating to respondents' security behaviours were identified as being awareness of threats, the presence of a catalyst event in the respondent's past, and a combination of knowledge and perceived self-efficacy that led to a willingness

$1 \quad$ Kervyn, et al. (2009) use Rosenberg, Nelson, and Vivekananthan's (1968) definition of a warm dimension to relate to "an underlying social good" (p. 363). 
and intention to engage in cybersecurity behaviours. These factors were important because of their influence both on other internal factors and on the other constructs included in the revised model. The revised model presented in Chapter 5 showed how awareness, perceived danger, internal factors, external factors, and perceived barriers contributed to respondents' intentions to engage in cybersecurity behaviours. In this chapter, the broadest implications of the revised model were discussed. Next, the value of examining users' security intentions and behaviours through the lens of user types was examined and related to existing literature on user types. Throughout the study, it was possible to see that there was a difference between how secure respondents thought they were compared to how secure they actually were. This built on existing research into the differences between awareness of threats and actually carrying out security behaviours. Finally, this chapter explored the effect of laziness on security behaviours. The nuances of laziness were explored in an attempt to determine why respondents preferred to consider themselves to be lazy about their security and what factors influenced this laziness. 
Braun 


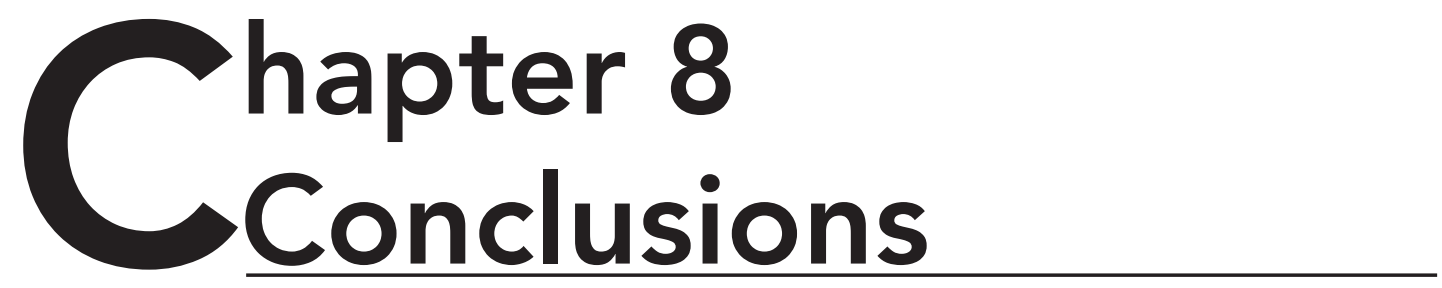

\subsection{Introduction}

This research has examined the factors that influence home users' intention to engage in cybersecurity practices. Focus groups and interviews were used to identify and explore these factors and their relationships. This research was undertaken from an interpretivist perspective. A qualitative approach was particularly useful in this study because of the lack of agreement by researchers on the factors that influence home users' cybersecurity behaviours.

While the qualitative approach yielded data about factors that have been studied in the existing cybersecurity literature, there were also several limitations associated with its use. Because of the nature of qualitative research, it is difficult to generalise the findings. Therefore, additional research based on these findings would be useful in the further exploration of the relationships between the factors and determining which factors most strongly influence users' cybersecurity behaviours. These issues are explored in further detail later in this chapter.

This chapter provides an overview of the findings in relation to the research questions from Chapter 1 regarding identifying the factors related to home users' intention to engage in security behaviours (Chapter 4) and the ways that these factors worked together to 
influence these intentions (Chapter 5). These findings will be related to the existing theory and the implications for theory, for practice, and for further research.

\subsection{Summary of Findings}

This research answers two research questions:

1. What are the factors influencing home users' cybersecurity behaviours?

2. How do these factors influence home users' cybersecurity behaviours?

To answer these questions, three research objectives were set:

1. Identify the factors affecting home user cybersecurity practices.

2. Develop a framework to explain how these factors influence home users' cybersecurity behaviours.

3. Determine how the factors relating to awareness of threats and safeguards can affect home users' cybersecurity practices.

A literature review was conducted to identify factors and models related to users' cybersecurity behaviours in both home and organisational contexts. From the literature review, it was determined that home users' cybersecurity behaviours had not been explored as deeply as organisational behaviours. Unlike organisational users, home users are not required to use security, nor are they required to undergo training to increase their awareness. Therefore, there was a gap in the understanding of what factors from organisational cybersecurity literature would be relevant in studying users' general cybersecurity behaviours. From the literature, a conceptual model was developed that included nine main constructs: perceived severity, perceived susceptibility, perceived threat, internal factors, external factors, demographic factors, perceived barriers and benefits, intention to engage in cybersecurity behaviours, and cybersecurity behaviours. The conceptual model was used to guide the data collection and in the initial stages of the data analysis.

To identify the relevant and additional factors influencing home users' security behaviours, focus groups and interviews were conducted. The analysis of the data from 
these led to the identification of six main constructs as well as their component factors that influenced home users' cybersecurity behaviours: awareness, internal factors, external factors, perceived danger, perceived barriers and enablers, and cybersecurity intentions. Once these factors were identified, a revised model was developed based on the relationships that were evident among these factors. In developing the revised model, several sub-factors were particularly influential in users' security behaviours. These factors combined in a way that made it possible to explore different types of users and their associated behaviours as a way to further illustrate how the factors interacted to influence intention to engage in safer behaviours. In the subsequent sections, a summary of the findings from each research question is presented along with relevant propositions based on the findings.

\subsubsection{Factors Influencing Home Users' Cybersecurity Intentions}

Research objective 1 sought to identify the factors that influence home users' cybersecurity behaviours. Six main constructs were identified that contributed to home users' cybersecurity intentions. Within these main constructs, further factors were also identified.

Awareness of threats is necessary for individuals to be able to form opinions about the danger of threats. Awareness focuses on identifying what threats individuals are conscious of rather than including their understanding of these threats. While understanding of threats and safeguards is relevant, it is not required for individuals to become alert to the existence of the threats in the news or through another source of information.

The construct of perceived danger includes individuals' beliefs about their susceptibility to threats and the severity of the associated consequences. Perceptions about severity and susceptibility can be tempered by how effective individuals believe that the safeguards they use are at protecting them from cybersecurity threats. If they believe that their safeguards are particularly effective, they are less likely to consider there to be a high level of danger from the threats because the safeguard is believed to limit their susceptibility to breaches and mitigate the associated damages.

External factors are cybersecurity related aspects of the individual's environment. Symptoms of breaches are the tangible signs that individuals see when they believe that their cybersecurity has been breached. These symptoms are often the catalyst event that triggers changes in security behaviours because they illustrate that the danger of threats is real. Without these symptoms, individuals are much less likely to believe that they are suffering from a security breach. Security messages provide individuals with information about threats that are pushed to them. In this way, security messages are particularly 
important because they do not require the individual to actively seek out information about threats and safeguards. Finally, whether or not an individual shares their computer with someone can contribute to their security intentions. In particular, if they share the computer and feel that someone else is taking care of their security, there is a tendency for individuals to take fewer protective steps. In contrast, if individuals believe that they are responsible for someone else's cybersecurity, they tend to take more protective steps.

Internal factors are comprised of the cognitive and affective factors unique to the individual. Past experiences with security threats is directly related to symptoms because the symptoms provide the catalyst event that triggers the emotional responses associated with security breaches. Knowledge of threats and safeguards builds on awareness by looking at how well individuals understand the threats and safeguards they are aware of. Perceived self-efficacy focuses on how confident individuals are in their ability to protect themselves from cybersecurity breaches. Reliance on others is used to describe how much responsibility individuals place on others to protect their cybersecurity. Although many individuals consider themselves to be responsible for their own cybersecurity, there is still an often demonstrated reliance on others. Trust in both websites and people is relevant to considering how individuals change their security behaviours if they consider a site or person to be trustworthy or how they change their behaviours if they believe that virtually nobody is trustworthy. Security orientation and concern about security includes how individuals felt about both safeguards and threats. While concern about threats is expectedly negative, feelings about safeguards are also often negative when they are considered to be inconvenient and annoying. Finally, ego protectiveness accounts for the feelings of laziness and boasting statements that individuals make, often to cover up a lack of concern or lack of knowledge about security.

The perceived barriers to security focus on the external barriers such as time, cost, and lack of prompting about security. Although factors such as lack of knowledge can be considered as barriers to engaging in security practices, this research shows that lower levels of certain internal factors tends to result in lower perceptions of danger, which acts similarly to barriers in how they influence cybersecurity intentions. The most influential external barrier to engaging in security is a lack of time. While enablers can overcome moderate perceptions of danger and positively contribute to intention to engage in safer practices, it is often difficult to identify clear benefits to being secure because the basis of security is to maintain the status quo. Therefore, barriers have a greater negative influence on users' intentions than the positive effect of enablers.

Finally, users' security behaviours are influenced by their intention to engage in the behaviours. The general assumption is that if individuals' have a strong enough intention to do something they will do so. However, within the cybersecurity context, it is possible 
to see that individuals will at times overstate the strength of their intention. This explains in part why so often we see individuals explaining that they know what they should be doing, even though they do not take those steps to protect their security. As a result, it is important to look to the factors that influence users' intention rather than assuming that their stated intention is accurate.

\subsubsection{Relationships between Factors Influencing Home Users' Cybersecurity Intentions}

Research objectives 2 and 3 focus on the relationships between the identified constructs and factors. Research objective 2 was to develop a framework to explain how the identified factors influence home users' security behaviours (see Figure 8-1). To form opinions about the severity of threats and their likely susceptibility to threats, the starting point emerged as awareness. In order to actually take step to prevent or mitigate the danger of these threats, users need to be aware of the possible safeguards they can use. Awareness of something is necessary before users can form opinions about it (Witte, 1992 as cited in Johnston \& Warkentin, 2010). Similar to Johnston \& Warkentin's findings, in this study, individuals who were unaware of the threats were less likely to consider them to be

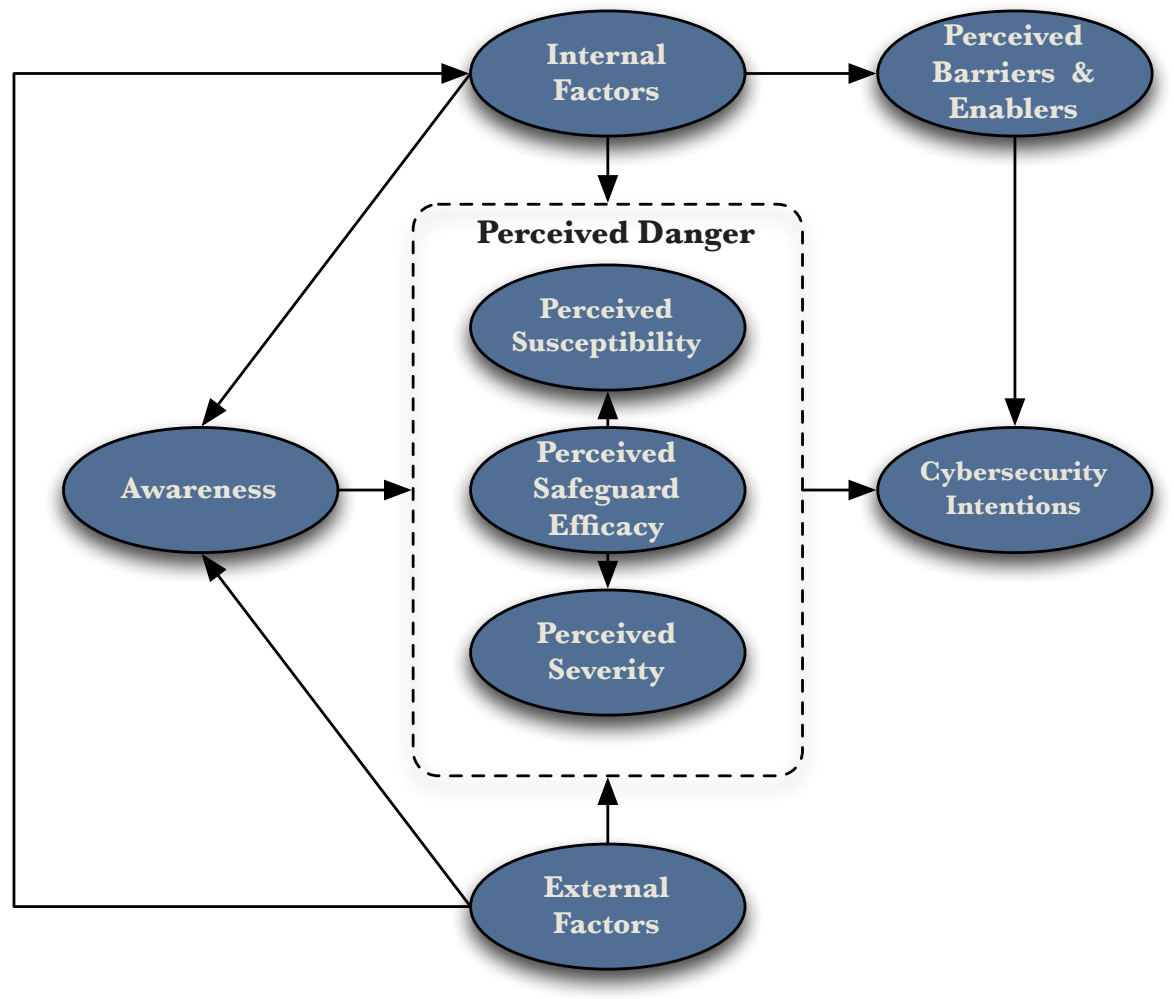

Figure 8-1. Revised model to explain how the identified factors and constructs affect home users' cybersecurity intentions 
dangerous. This is often because they do not have the necessary context for understanding the danger and instead assume that because they have not heard of the threat, there is no need to be concerned. Awareness of threats is influenced by internal factors and external factors. The components of the internal factors are positively and negatively associated with awareness.

Proposition 1a: The internal factor components of knowledge, security orientation, and prior experience are positively associated with awareness of threats.

Proposition 1b: The internal factor components of reliance and trust are negatively associated with awareness of threats.

Proposition 1c: The internal factor component of perceived self-efficacy is associated with awareness of threats.

Similar to how individuals with lower levels of awareness lack the necessary context for creating opinions on security, individuals with lower levels of these factors are more likely to consider the chances of suffering from a breach to be low and the consequences to be minor. This is because they either do not know what the possible consequences are or because they do not think they can prevent them.

Awareness of threats is also influenced by external factors. External factors such as symptoms and security messages provide individuals with the necessary evidence of the existence threats, either because they have experienced the consequences for themselves or because they have been given information about the threats from relevant sources. As a result, they can serve to increase awareness of threats. However, security messages only increase threat awareness if (1) the person understands the message and (2) they believe that the message is relevant.

Proposition 2a: The external factor component of symptoms is positively associated with threat awareness.

Proposition 2b: The external factor components of security messages and computer use context are associated with threat awareness.

However, simply being aware of threats does not mean that people will act on this awareness. 
In order for individuals to act on the threats that they are aware of, they must consider the danger to be high enough to prompt a change in their behaviours. While awareness serves as a necessary precursor to developing perceptions of danger, it is not the only factor that influences it.

Proposition 3: Awareness of threats is positively associated with perceived danger of threats.

Perceptions of danger are further influenced by respondents' internal and external factors. Internal factors influence not only their awareness of threats, but also their perceptions of danger. In particular, individuals' perceived and actual knowledge, perceived self-efficacy, security orientation, and their past experiences with threats have the greatest effect on their perceptions of danger. Lower levels of these factors tend to result in lower levels of perceived danger.

Proposition 4a: The internal factor components of knowledge, security orientation, and prior experience are positively associated with perceived danger.

Proposition 4b: The internal factor components of reliance, perceived self-efficacy, ego protection, and trust are negatively associated with perceived danger.

External factors also serve to enhance perceptions of danger because just as they provide evidence to prompt awareness, they also provide evidence that they danger is relevant to the individual. As a result, symptoms provided the greatest role for increasing the perception of danger.

Proposition 5a: The external factor components of symptoms and computer use context are positively associated with perceived danger.

Proposition 5b: The external factor component of security messages is associated with perceived danger.

Similar to what was seen with awareness, security messages have a positive influence if the individual considers them to be relevant and they understand them. In contrast, if they are viewed as irrelevant or are not understood, the result can be neutral or even negative because individuals do not consider themselves to be susceptible to the threat. 
As can be seen from the relationship between security messages and knowledge, there are also links between internal and external factors.

Proposition 6a: The external factor component of symptoms is positively associated with the internal factor components of prior experience, security orientation, and knowledge.

Proposition 6b: The external factor component of symptoms is negatively associated with the internal factor component of trust.

Proposition 6c: The external factor component of computing context is associated with the internal factor components of trust, reliance, perceived self-efficacy, and knowledge.

Proposition 6d: The external factor component of security messages is associated with perceived self-efficacy.

This shows how what happens in an individual's external environment can have greater reaching consequences on individual security behaviours through their internal factors. For example, if people experience consistent and early success in dealing with security breaches they may be more confident in their ability to prevent or remedy them in the future. However, if an individual has a more negative experience where they lose data or are unable to remedy the breach, this may have a more negative effect and decrease their perceived ability to prevent cybersecurity breaches, regardless of any evidence to the contrary.

Conclusions about how dangerous the threats are leads to intentions to engage in security behaviours.

Proposition 7: Perceived danger is positively associated with cybersecurity intentions.

The greater the perceived danger of the threat, the more likely an individual is to want to take steps to avoid the threat. However, if the threat is not considered to be dangerous or relevant to the individual, they are less likely to intend to take steps to protect themselves.

Although perceptions of danger play a strong role in influencing users' intentions 
and in turn, their cybersecurity behaviours, their intention can be further influenced by barriers and enablers of security. The barriers and enablers of security are influenced individuals' internal factors.

Proposition 8a: The internal factor components of security orientation, knowledge, prior experience, and perceived selfefficacy are negatively associated with perceived barriers.

Proposition 8b: The internal factor components of reliance, ego protection, and trust are positively associated with perceived barriers.

Proposition \&c: The internal factor components of security orientation, knowledge, prior experience, and perceived selfefficacy are positively associated with perceived enablers.

Proposition 8d: The internal factor components of reliance, ego protection, and trust are negatively associated with perceived enablers.

Although both barriers and enablers need to be considered, it may be difficult to qualify what enables security behaviours, especially in the absence of perceived danger. Individuals may find it easier to qualify why they do not engage in security behaviours rather than identifying reasons why they do. Although enablers were expected to have a somewhat greater effect on intention to engage in safer behaviours, it was expected that individuals would be more aware of barriers since the goal of security is to keep everything as it should be.

From this research, it appears that the main factors that influence home users' cybersecurity intentions are perceived danger and the barriers to engaging in security behaviours. Barriers and enablers are of particular importance if an individuals' perceptions of danger are more moderate since they can shift the balance towards engaging or not engaging in protective behaviours.

Proposition 9a: Perceived barriers are negatively associated with cybersecurity intentions. 
Braun

Proposition 9b: Perceived enablers are positively associated with cybersecurity intentions.

The result is that the combination of perceived danger and perceived barriers and benefits lead to a user's cybersecurity intention.

Finally, users' intentions were shown to influence their overall behaviours.

Proposition 10: Cybersecurity intentions are positively associated with cybersecurity behaviours.

Although respondents often made statements that implied that they intended to protect their computer, it was clear that in many cases this was not always the case. In these situations it is necessary to look back to the perceptions of danger and the barriers and enablers to see if the perceived intention matches the actual intention. However, if the actual intention is strong enough, the users' intentions are a good predictor of their overall cybersecurity behaviours.

\subsubsection{The Gap between Awareness and Practice}

Further addressing research objective 3, which is to determine how the factors relating to awareness of threats and safeguards can affect home users' cybersecurity practices, the gap between awareness and practice is explored. One of the issues that has been identified in research into home users' security behaviours is the existence of a gap between what people know and what they actually do to protect themselves. In many cases, respondents indicated that they were "too lazy" to protect their cybersecurity (see section 7.5). However, many of these same respondents indicated an awareness that they not only needed to protect their security, but also that they were aware of at least the basic steps they could be taking. This research has provided a starting point for understanding why this gap exists.

Laziness is a common reason cited for people not doing more to protect themselves. However, the glibness and speed with which these responses were provided indicated that there is a need to further examine what causes people to consider themselves to be lazy. Laziness was an almost immediate reaction when respondents were asked why they did not take the additional steps that they knew of. However, further questioning showed that in many cases it was not laziness that was responsible for their inaction. Instead it often stemmed from other reasons. Therefore, the starting point for exploring this self-diagnosed laziness about security is to look at what factors were present for individuals who consider themselves to be lazy.

Often, laziness is used as a way to distract from a lack of knowledge combined with 
a lack of concern about security. Respondents seemed to prefer considering themselves to be lazy rather than unknowledgeable. There was a general consensus that everyone "knew" about security because the issue is so universal to our culture with the ubiquity of the internet. A comparison of the baseline level of knowledge that people are assumed to have with the knowledge that people actually demonstrated, suggests that respondents really do not want to be seen as unknowledgeable despite earlier comments about their lack of knowledge. Although laziness is often used to distract from a lack of knowledge, even some of the most knowledgeable respondents considered themselves to be lazy about their security. Therefore, knowledge, and a lack thereof, did not appear to be the sole driver of the gap between what respondents knew and what they did.

A lack of concern about security also contributes to the use of laziness as an excuse for not engaging in more protective behaviours. This lack of concern often stems from a lack of perceived danger about the threats. Assessments of perceived danger are often rooted in knowledge and awareness of threats. If individuals have low levels of awareness or are not particularly knowledgeable about threats, they do not have the necessary starting point from which to form opinions about their susceptibility to threats or the severity of the potential consequences. However, even individuals who are knowledgeable can feel that the overall danger is low because they believe they are not susceptible to threats. The reasons for this range from lack of anything of value, to not being the right kind of target, to trusting in the steps that they had already taken to prevent threats. However, without the perceptions of danger, there is little to drive individuals to overcome their "laziness." In reality, this laziness is more closely linked to a lack of perceived danger which in turn contributes to lower intentions to engage in protective behaviours.

Although knowledge and perceived susceptibility appear to be the main contributors to laziness and the gap between knowledge and intention, it can be the result of any number of combined factors that lead to lower levels of perceived danger. Therefore, in order to address the issue of laziness surrounding people's intentions to engage in protective behaviours, there is a need to address the factors that influence perceived danger. One way that this can be addressed is to look at the combinations of factors, as used to determine user types, that influence users' security.

\subsection{Implications}

In this section, the theoretical and practical implications for this study are presented. The main theoretical contribution is the addition of the model to the body of knowledge and an increased understanding of the reasons why the gap between awareness and knowledge exists. The main practical contribution is that the understanding of the factors 
that influence users' intentions to engage in protective behaviours can be used to create more effective targeted security messages.

\subsubsection{Theoretical Implications}

Although factors influencing home users' cybersecurity behaviours have been identified in past research (see Table 2-5, p. 51-52), the list of factors is far from exhaustive. In particular, there is a gap in the knowledge about the factors that influence users' awareness of threats and the value of awareness provides in relation to users' intention to engage in protective behaviours. The current research contributes to the body of knowledge on our understanding of users' cybersecurity behaviours and what factors contribute to their intentions to engage in these behaviours. In particular, this research has looked at home users' general security behaviours rather than the specific use of a particular technology, which is an area where much of the previous research has been focused.

This research has shown how several factors that have not been examined in great detail in previous research are relevant to users' cybersecurity behaviours. In particular, the awareness of threats has not been extensively researched. In many cases, awareness appeared to be assumed because of the focus on particular technologies or threats. In the context of general security behaviours taken in this study, awareness has been shown to be a precursor to the development of perceptions about the danger of threats. Few previous cybersecurity studies have considered awareness as a distinct factor or construct. Nor have previous cybersecurity studies considered the factors that can lead to increased awareness. In this study, the factors influencing awareness have been explored and it is possible to see how both internal and external factors can contribute to users' awareness of threats and safeguards. In addition, this research has shown how awareness is a prerequisite for developing opinions about the danger of threats. This further supports the proposition by Chen and Bansal (2010) that awareness, along with end user self efficacy and security skill, contributes to perceived security risk, which leads to intention to transact with a website.

In addition, this research also looks not only at how factors relate to users' cybersecurity behaviours, but also how these factors influence each other. While other health-based cybersecurity research has looked at how several main factors influence users' intentions, it has not considered the relationships between these constructs or the relationships between the component factors. Much of the existing research has looked at examining factors related to cybersecurity in isolation. However, this research contributes to the body of knowledge about how the constructs influence each other and contribute to users' cybersecurity intentions, but also how the factors within the constructs can affect each other. 
Although researchers have identified the gap between what people know and what they do, there has been a consistent need to identify what causes this gap. The identification of the factors found in this research contribute to further understanding not only about whether people actually know how to protect their security, but why they do not. This research suggests that one of the main reasons why people do not follow through security even though they should know better is because they do not consider the danger of the threats to be high enough. Severity and susceptibility are the main components of perceived threat and have long been established as relevant factors when studying cybersecurity behaviours. However, a gap remained in explaining why perceptions of severity and susceptibility were not sufficient to influence users' intentions to protect themselves. While other factors have been studied alongside of severity and susceptibility, there has been little research into understanding what contributes to these perceptions. This research begins to address the gap on identifying what factors contribute to perceptions of severity and susceptibility by exploring the relevant internal and external factors and how they affect perceived danger along with awareness.

Finally, while categorising individuals based on characteristics has often been used in marketing research, researchers have only begun to expand on how users could be classified based on their cybersecurity behaviours. Therefore, the main contribution to theory from the identification of user types, is as a starting point for determining how certain factors interact and contribute to users' cybersecurity behaviours. Although user types have been used minimally in categorising users (see section 7.4), they have not been used in this context or in this way. Therefore, the identification of the user types offers an additional level of explanation and clarity for explaining what factors contribute to users' cybersecurity behaviours and how these factors work together to influence users' cybersecurity behaviours.

\subsubsection{Practical Implications}

The value of identifying these factors in practical terms is for the companies and people whose job it is to promote internet security. Practically, the examination of what threats users are aware of (see section 4.3) highlights a difference between what people know about and what security companies think that users should be concerned about. This highlights a challenge for security companies who may assume that users have a certain base level of awareness of threats, which may not actually be present.

Another message that can be taken away from this research is that users need to be aware of both the threats and the safeguards in order to form opinions about the perceived danger. The understanding and awareness of threats contributes to perceived severity and 
susceptibility. Awareness and understanding of safeguards can contribute to perceived severity and susceptibility as well as beliefs about the effectiveness of safeguards. This means that if we want to ensure that users form accurate opinions about cybersecurity threats, they need to both be aware of the threats and safeguards and have enough of an understanding of the threats to assess the relevance to them personally.

Building on the importance of the individual factors in improving the security messages that companies and organisations put forward, the model provides further insight into which factors can be targeted in particular for the greatest benefit. As indicated earlier, the user types highlight areas where particular groups of users have factors that contribute to lower perceptions of danger or that decrease their intention to engage in cybersecurity practices. Therefore, understanding which factors contribute to users' intentions and where there may be deficiencies can contribute to more effective security messages and software. One of the biggest issues that has been seen in both this research and in the literature is that with security, there is a risk that people will feel that the threat does not apply to them (Johnston \& Warkentin, 2010). Targeted security messages have been shown to be more influential in modifying users' behaviours (see Ng et al., 2008; Witte \& Allen, 2000). Using the identified security types provides a way for not only providing targeted messages based on the users' behaviours, but also a starting point for figuring out how to increase the relevance of security messages to people who may otherwise not see the benefit of engaging in cybersecurity behaviours.

In relation to practice, the identification of the gap between awareness and practice provides a starting point for looking at the areas we need to improve in order to minimise that gap. One of the main findings was that respondents often did not fully understand what the actual threat was and because of this, tended to find reasons to justify why they did not need to be concerned. This suggests that there are areas where individuals need to become more knowledgeable before they can be expected to understand what the dangers are online.

Finally, as cybersecurity breaches become more common, it seems likely that users will be expected to have some base level of knowledge about what they need to protect against and how they can do so. However, if there is an assumption that users already have this knowledge, there is a danger that people are not being provided with the type of information they need not only to protect their own home computers, but also to avoid being a pathway for harming other systems. Therefore, in figuring out what factors contribute to the gap between awareness and practice, more targeted messages can be used, which may help overcome that gap. 


\subsection{Limitations}

One of the main limitations identified in regards to qualitative research is that it is not generalisable. However, Barbour (2001) suggests the aim of qualitative research should be to "reflect the diversity within a given population" (p. 1115) rather than focusing on generalisability. In this study, purposive sampling was used in order to explore a variety of respondents' perceptions and behaviours in relation to their cybersecurity. Three focus groups and 20 individual interviews were carried out. Interviews were conducted until no new information emerged in order to ensure that a broad enough selection of participants was covered. In addition, participants were selected in order to fulfil a broad selection of user types (focusing on age, gender, and the length of time they had been using the internet). Although the original intention was to get a spread of users who had been using the internet for different lengths of time, almost all participants had used the internet for $10+$ years. Few people approached had used it for less time. This was likely due to the fact that New Zealand is a country where over $75 \%$ of the population has access to the internet (New Zealand Ministry of Social Development, 2010).

Despite not being as generalisable as quantitative data, for the purposes of this study, qualitative research remained appropriate. The goal of this study was not to test the relationships between identified constructs, but rather to determine if the existing constructs were appropriate and if additional constructs and relationships needed to be identified and explored. Although certain factors were already identified as being relevant to influencing organisational users' cybersecurity behaviours, there is still little research focusing on the home user. This combined with the number of people affected by cybersecurity breaches suggests that we have not yet figured out a way to address the gap between awareness and practice. Therefore, despite existing research into users' cybersecurity behaviours, there remained a need to identify factors and relationships between factors that contribute to users' security behaviours. In order to address the limitation of being generalisable, in the future, additional quantitative research should be carried out in order to further verify the relevance and relationships of the identified factors.

The focus on users' intentions rather than their actual behaviours could be considered a limitation. However, as discussed in Chapter 2, if an individual has a strong enough intention to do something, there is an assumption that the person will do so. Therefore, it is possible to study intentions rather than behaviours.

A similar limitation is that this study focused on perceptions, such as perceived awareness or perceived knowledge. There may be a difference between what people perceive the reality to be and the actual reality. However, much of the way that individuals act is governed by their perception of reality. Therefore, even if respondents' perceptions did not 
represent an objective view of reality, it is their subjective interpretation that will guide their actions. Although the identified factors could be tested quantitatively to determine actual levels of knowledge or awareness, there is value in exploring users' perceptions of their own ability. Perceptions may be valuable in exploring how users' see themselves especially in terms of security given the existence of the gap between what people know and what they do. If their actual awareness or knowledge was tested in a quantitative way, it may not have provided a complete picture of the what factors influenced users.

Finally, interpretative research by its very nature is open to some degree of variance based on the person interpreting the statements. Therefore, although this is a limitation to the study, the model presented can be further refined and validated through the use of quantitative research.

\subsection{Areas for Future Research}

The model presented in this research can be refined using quantitative research in order to further explore the relationships between the identified factors. Although relationships have been proposed based on the qualitative data, these relationships could be further tested in order to help establish whether the model can be further generalised to a wider context.

Another area for future research with this model is to explore the applicability of it within an organisational context. Although organisations are likely to have clearer rules regarding the use of the internet and sanctions for acting outside of those rules, it remains that security is the responsibility of people and in order to encourage more secure behaviours amongst workers, many of these factors may continue to be relevant.

This research shows how several factors appear to contribute to the gap between awareness, knowledge, and practice. However, future research could be done to explore if there are additional factors contributing to this gap, and to find out which factors are most influential. Furthermore, additional research could be done in looking at the effectiveness security training and messages that address factors contributing to the gap are in influencing users' to engage in more secure behaviours online.

This research indicates a need for additional research into how the differences between perceived and actual levels of awareness and knowledge contribute to the gap between what people say they know compared to what they do. From the research, it appears that the differences between perceived and actual levels of these factors contribute to the overall behaviours that users engage in. However, more could be done to quantitatively assess the actual knowledge and awareness of threats to be compared to perceived levels.

The user types identified in this study can be further refined and reviewed to explore 
their applicability in a wider context of both home and organisational users. Furthermore, they can also be further researched in order to determine if there are additional relevant factors, how the factors relate to each other, and the strengths of the relationships between the factors.

\subsection{Conclusions}

This research has taken a qualitative, exploratory approach to studying home users' internet security behaviours. In doing so, the factors relating to home users' cybersecurity behaviours have been identified and a model has been developed based on these factors in order to illustrate how the factors interact. Furthermore, based on the data, five main types of users were identified in relation to their features and their security behaviours. These user types helped to further illustrate the relationships between the factors shown in the model as well as to illustrate how the factors can combine and lead to certain behaviours.

This research further explores the realm of home users' cybersecurity behaviours, an emerging topic in the field and builds on the existing literature. Awareness was shown to have had a prominent role in influencing users' behaviours. Without awareness they do not have the necessary contextual understanding of the threats they are faced with, and as a result have a much more difficult time evaluating their subjective susceptibility to the threats and the severity of the consequences. However, awareness alone does not ensure that users will act safely. Instead, a combination of internal and external factors contributes to the perceived danger which, in combination with the barriers to engaging in security behaviours, leads to users' cybersecurity intentions.

With users' behaviours, the gap between awareness and practice was particularly relevant. This gap has been identified in previous literature, but has needed further exploration to help identify what factors contribute to its existence. One of the ways to address this gap is to focus on the factors that influence users' perceptions when creating security messages. Targeted security messages have been shown by researchers to be more effective in modifying behaviours. In order to address the combination of factors that contribute to the gap, the user types can be used to focus on the combination of factors that are particularly relevant for each of the types of users, creating even more targeted messages.

This chapter has provided a summary of the main findings of the research. It has also elaborated on the implications for theory and practice and identified areas for future research in relation to each of the main findings. 
Braun 


\section{Chapter 9 References}

Adams, A., \& Sasse, M. A. (1999). Users are not the enemy. Communications of the Association for Information Systems, 42(12), 40-46. doi:10.1145/322796.322806

Adhikari, R. (2012). E-Commerce news: Enterprise security: Hacktivists, not profiteers, stole most data in 2011. ECommerce Times. Retrieved from http://www.ecommercetimes. com/story/74700.html

AFP. (2012). Hacktivists the biggest data thieves in 2011: Report. Sydney Morning Herald. Retrieved from http://www.smh.com.au/action/printArticle?id=3156922

Ajzen, I. (1991). The theory of planned behavior. Organizational Behavior and Human Decision Processes, 50(2), 179-211. doi:10.1016/0749-5978(91)90020-T

Albrechtsen, E. (2007). A qualitative study of users' view on information security. Computers E Security, 26(4), 276-289. doi:10.1016/j.cose.2006.11.004

Anderson, C. L., \& Agarwal, R. (2010). Practicing safe computing: A multimethod empirical examination of home computer user security behavioral intentions. MIS Quarterly, 34(3), 613-643.

Anti-Phishing Group at Indiana University. (2006). Stop-phishing.com. Retrieved from http://www.indiana.edu/ phishing/?faq

AOL/NCSA. (2005). AOL/SCSA Online Safety Study. Retrieved from http://www. staysafeonline.org/pdf/safety_study_2005.pdf 
Armitage, C. J., \& Conner, M. (2001). Efficacy of the theory of planned behaviour: A meta-analytic review. British Journal of Social Psychology, 40(4), 471-499. doi:10.1348/014466601164939

Armstrong, D., Gosling, A., Weinman, J., \& Marteau, T. (1997). The place of inter-rater reliability in qualitative research: An empirical study. Sociology, 31(3), 597-606. doi:10.1177/003803859703100301

Ashford, W. (2013). Malware in counterfeit software to cost businesses $\$ 114 \mathrm{bn}$ in 2013. Computer Weekly. Retrieved from http://www.computerweekly.com/ news/2240180104/Malware-in-counterfeit-software-to-cost-business-144bnin-2013

Australian Communications and Media Authority. (2007). Developments in internet filtering technologies and other measures for promoting online safety. Developments in Internet Filtering Technologies and Other Measures for Promoting Online Safety. Retrieved from http://www.acma.gov.au/webwr/_assets/main/lib310554/ developments_in_internet_filters_2ndreport.pdf

AVG Anti-Virus and Internet Security. (2008). National Economies Threatened by Cybercrime, According to EU Information Security Agency. Retrieved from www. webwire.com/ViewPressRel.asp?aId=67560

Aytes, K., \& Connolly, T. (2004). Computer security and risky computing practices: A rational choice perspective. Fournal of Organizational and End User Computing, 16(3), 22. doi:10.4018/978-1-59904-937-3.ch263

Bandura, A. (1977). Self-efficacy: Toward a unifying theory of behavioral change. Psychological Reviere, 84(2), 191-215. doi:10.1037//0033-295X.84.2.191

Bandura, A. (1982). Self-efficacy mechanism in human agency. American Psychologist, 37(2), 122-147. doi:10.1037//0003-066X.37.2.122

Bandura, A. (1986). Social foundations of thought and action: A social cognitive theory. Englewood Cliffs, NJ: Prentice-Hall.

Barbour, R. S. (2001). Checklists for improving rigour in qualitative research: A case of the tail wagging the dog. British Medical fournal, 322, 1115-1117. doi:10.1136/ bmj.322.7294.1115

Barnes, S. J., Bauer, H. H., Neumann, M. M., \& Huber, F. (2007). Segmenting cyberspace: A customer typology for the internet. European fournal of Marketing, 41(1/2), 71-93. doi:10.1108/03090560710718120

Basch, C. E. (1987). Focus group interview: An underutilized research technique for improving theory and practice in health education. Health Education Quarterly, 14(4), 411-448. doi:10.1177/109019818701400404 
Becker, M. H., Maiman, L. A., Kirscht, J. P., Haefner, D. P., \& Drachman, R. H. (1977). The health belief model and prediction of dietary compliance: A field experiment. Journal of Health and Social Behavior, 348-366. doi:10.2307/2955344

Berkowitz, B., \& Hahn, R. W. (2003). Cybersecurity: Who's watching the store? Issues in Science and Technology, 19(3), 55-62.

Bishop, M. (2003). What is computer security? IEEE Security and Privacy, 1(1), 67. doi:10.1109/ MSECP.2003.1176998

BizTech2.com Staff. (2013). 2012 global losses from phishing estimated at \$1.5 bn. Retrieved from http://biztech2.in.com/news/security/2012-global-losses-fromphishing-estimated-at-\$15-bn/154212/0

Blanton, H., Axsom, D., McClive, K. P., \& Price, S. (2001). Pessimistic bias in comparative evaluations: A case of perceived vulnerability to the effects of negative life events. Personality and Social Psychology Bulletin, 27(12), 1627-1636. doi:10.1177/01461672012712006

California State Parks. (2009). Coyote's place in Native American stories. Retrieved from http://www.parks.ca.gov/pages/23071/files/coyotesplace_3up_finallayout072709. pdf

Center for Strategic and International Studies. (2008). CSIS Commission on Cybersecurity for the 44th Presidency. Retrieved from http://csis.org/files/media/csis/ pubs/081208_securingcyberspace_44.pdf

Chan, M., Woon, I., \& Kankanhalli, A. (2005). Perceptions of information security in the workplace: Linking information security climate to compliant behavior. Journal of Information Privacy \& Security, 1(3), 18.

Charoen, D., Raman, M., \& Olfman, L. (2008). Improving end user behaviour in password utilization: An action research initiative. Systemic Practice and Action Research, 21(1), 5572. doi:10.1007/s11213-007-9082-4

Chen, L. G., \& Bansal, G. (2010). An integrated model of individual web security behavior. Proceedings of the Sixteenth Americas Conference on Information Systems. Retrieved from http:/ / aisel.aisnet.org/amcis2010/484

Chmielewski, D. C. (2012). 'The hunger games' used as bait by hackers. LA Times. Retrieved from http://latimesblogs.latimes.com/entertainmentnewsbuzz/2012/03/hungergames-katniss-used-as-bait-by-hackers.html

Chou, W. (2007). Cybersecurity costs: Balancing blanket security with real-world practicality. IT Professional, 9(2), 16. doi:10.1109/MITP.2007.30

Chua, W. F. (1986). Radical developments in accounting thought. The Accounting Review, 61(4), 601-632. doi:10.1016/B978-008044725-4/50009-6 
Cluley, G. (2012). Server-side polymorphism: How mutating web malware tries to defeat anti-virus software. NakedSecurity. Retrieved from http://nakedsecurity.sophos. com/2012/07/31/server-side-polymorphism-malware/

Compeau, D. R., \& Higgins, C. A. (1995a). Application of social cognitive theory to training for computer skills. Information Systems Research, 6(2), 118-143. doi:10.1287/ isre.6.2.118

Compeau, D. R., \& Higgins, C. A. (1995b). Computer self-efficacy: Development of a measure and initial test. MIS Quarterly, 19(2), 189-211. doi:10.2307/249688

Computer Economics. (2007). Annual Worldwide Economic Damages From Malware Exceed \$13 Billion. Retrieved from http://www.computereconomics.com/article. cfm?id= 1225

Corrons, L. (2013). A look back at cyber-security in 2012. Panda Labs. Retrieved from http://pandalabs.pandasecurity.com/a-look-back-at-cyber-security-in-2012/

Crafty, G. (2009). Internet Crime Jumps by a Third Last Year. CNN. Retrieved from http:/ / edition.cnn.com/2009/TEGH/03/30/internet.crime/index.html

Creswell, J. W. (2003). Research design: Qualitative, quantitative, and mixed methods approaches (2nd ed.). Thousand Oaks, CA: Sage Publications Inc.

Culnan, M. J., Foxman, E. R., \& Ray, A. W. (2008). Why IT executives should help employees secure their home computers. MIS Quarterly Executive, 7(1), 49-56.

Cyber Secure Institute. (2009). Cyber Secure Institute on the Conficker Controversy. Retrieved from http://cybersecureinstitute.org

Davinson, N., \& Sillence, E. (2010). It won't happen to me: Promoting secure behaviour among internet users. Computers in Human Behavior, 26(6), 1739 - 1747. doi:10.1016/j. chb.2010.06.023

Davis, F. D., Bagozzi, R. P., \& Warshaw, P. R. (1989). User acceptance of computer technology: A comparison of two theoretical models. Management Science, 35(8), 9821003. doi:10.1287/mnsc.35.8.982

Department of Homeland Security. (n.d.). National cyber security awareness month. Retrieved from http://www.dhs.gov/national-cyber-security-awareness-month

Dinev, T., \& Hu, Q. (2007). The centrality of awareness in the formation of user behavioral intention toward protective information technologies. Fournal of the Association for Information Systems, 8(7), 386-408.

Dommeyer, C. J., \& Gross, B. L. (2003). What consumers know and what they do: An investigation of consumer knowledge, awareness, and use of privacy protection strategies. Fournal of Interactive Marketing, 17(2), 34-51. doi:10.1002/dir.10053 
Dotinga, W. (2013). Botnet genius stole $\$ 500$ million in global scam, Microsoft says. Courthouse Newes Service. Retrieved from http://www.courthousenews.com/2013/06/10/58358. htm

Drennan, J., Mort, G. S., \& Previte, J. (2006). Privacy, risk perception, and expert online behavior: An exploratory study of household end users. Fournal of Organizational and End User Computing, 18(1), 1.

Eichorn, K., \& Fucanan, C. (2012). Consumer alert: McAfee finds one in every six personal computers have zero protection. Retrieved from http://www.mcafee.com/hk/ about/news/2012/q2/20120530-01.aspx

Eitle, D., \& Taylor, J. (2008). Are hispanics the new "threat"? Minority group threat and fear of crime in Miami-Dade county. Social Science Research, 37(4), 1102-1115. doi:10.1016/j.ssresearch.2008.05.005

Ferraro, B., \& Parayno, J. (2008). Cyber crime feared more than burglary, new study suggests. PR Neweswire. Retrieved from http://www.prnewswire.com/news-releases/ cyber-crime-feared-more-than-burglary-new-study-suggests-57426307.html

Fishbein, M., \& Ajzen, I. (1975). Belief, attitude, intention, and behavior : An introduction to theory and research. Reading, Massachusetts: Addison-Wesley.

Fishbein, M., \& Cappella, J. N. (2006). The role of theory in developing effective health communications. Fournal of Communication, 56(s1), s1-s17. doi:10.1111/j.14602466.2006.00280.x

Fiske, S. T., Cuddy, A. J., \& Glick, P. (2007). Universal dimensions of social cognition: Warmth and competence. Trends in Cognitive Sciences, 11(2), 77-83. doi:10.1016/j. tics.2006.11.005

Furnell, S. M., Bryant, P., \& Phippen, A. D. (2007). Assessing the security perceptions of personal internet users. Computers \& Security, 26(2007), 410-417. doi:10.1016/j. cose.2007.03.001

Furnell, S. M., Tsaganidi, V., \& Phippen, A. D. (2008). Security beliefs and barriers for novice internet users. Computers EO Security, 27, 235-240. doi:10.1016/j.cose.2008.01.001

Gallagher, S. (2012). Born to be breached: The worst passwords are still the most common. Arstechnica. Retrieved from http://arstechnica.com/informationtechnology/2012/11/born-to-be-breached-the-worst-passwords-are-still-the-mostcommon/

Garrison, G. P. (2008). An evaluation of passwords. The CPA Fournal, 78(5), 70-71.

Gibbs, A. (1997). Focus groups. Social Research Update, 19. doi:10.1111/j.13652788.2008.01057.x 
Glanz, K., Rimer, B. K., \& Lewis, F. M. (2002). Health behaviour and health education. Theory, research and practice. San Francisco, California: Wiley \& Sons.

Golafshani, N. (2003). Understanding reliability and validity in qualitative research. The Qualitative Report, 8(4), 597-607.

Goldsborough, R. (2007). How serious a threat are computer viruses? Tech Directions, 67(1), 14-15.

Good, N., Grossklags, J., Thaw, D., Perzanowski, A., Mulligan, D., \& Konstan, J. (2006). User choices and regret: Understanding users' decision process about consensually acquired spyware. I/S: A Journal of Law and Policy for the Information Society, 2(2), 283344.

Goodin, D. (2012). Mushrooming ransomware not extorts $\$ 5$ million a year. arstechnica. Retrieved from http://arstechnica.com/security/2012/11/mushrooming-growthof-ransomware-extorts-5-million-a-year/

Goodin, D. (2013). Strange "ransomware" title pushes surveys, knows Close Encounters tune. arstechnica. Retrieved from http://arstechnica.com/security/2013/07/strangeransomware-title-pushes-surveys-knows-close-encounters-tune/

Gregor, S. (2006). The nature of theory in information systems. MIS Quarterly, 30(3), 611 642.

Grossman, W. M. (2007). Does antivirus have a future? The Guardian, p. 1. Retrieved from http:// www.guardian.co.uk/technology/2007/sep/20/guardianweeklytechnologysection. spam

Guzik, H. (2013). Change your password day: Are you hackable or uncrackable? Intel IQ. Retrieved from http://iq.intel.com/iq/32516800/change-your-password-day-isyour-password-hackable

Harrington, S., Anderson, C. L., \& Agarwal, R. (2006). Practicing safe computing: Message framing, self-view, and home computer user security behavior intentions. Proceedings of the Twenty-Seventh International Conference on Information Systems, Milwaukee, Wisconsin,1543-1562. Retrieved from http://aisel.aisnet.org/icis2006/93

Helmreich, S. (2000). Flexible infections: Computer viruses, human bodies, nationstates, evolutionary capitalism. Science, Technology \& Human Values, 25(4), 472-491. doi:10.1177/016224390002500404

Holoien, D. S., \& Fiske, S. T. (2012). Downplaying positive impressions: Compensation between warmth and competence in impression management. Fournal of Experimental Social Psychology, 49(1). doi:10.1016/j.jesp.2012.09.001 
Honan, M. (2012). How Apple and Amazon security flaws led to my epic hacking. Wired. Retrieved from http://www.wired.com/gadgetlab/2012/08/apple-amazon-mathonan-hacking/

Internet Storm Center. (2013). Survival Time. Retrieved from https://isc.sans.edu/ survivaltime.html

Internet World Stats. (2011). World Internet Users and Population Statistics. Retrieved from http://www.internetworldstats.com/stats.htm

Jackson Higgins, K. (2008). Secunia: Less Than 2 Percent of PGs Are Fully Patched, Protected. Dark Reading. Retrieved from http://www.darkreading.com/end-user/ secunia-less-than-2-percent-of-pcs-are-f/212201991

Jackson Higgins, K. (2009). No User Action Required in Newly Discovered PDF Attack. Dark Reading. Retrieved from http://www.darkreading.com/attacks-breaches/nouser-action-required-in-newly-discove/215801319

Janz, N. K., \& Becker, M. H. (1984). The health belief model: A decade later. Health Education Behavior, 11(1), 1-47. doi:10.1177/109019818401100101

Jarvenpaa, S. L., Tractinsky, N., \& Vitale, M. (2000). Consumer trust in an internet store. Information Technology and Management, 1(1-2), 45-71. doi:10.1111/j.1083-6101.1999. tb00337.x

Johnston, A. C., \& Warkentin, M. (2010). Fear appeals and information security behaviors: An empirical study. MIS Quarterly, 34(3), 549-566.

Junglas, I. A., Johnson, N. A., \& Spitzmüller, C. (2008). Personality traits and concern for privacy: An empirical study in the context of location-based services. European fournal of Information Systems, 17(4), 387-402. doi:10.1057/ejis.2008.29

Kaspersky Lab. (2008). Kapersky lab reports a new and dangerous blackmailing virus. Retrieved from http://www.kaspersky.com/about/news/virus/2008/Kaspersky_ Lab_reports_a_new_and_dangerous_blackmailing_virus

Kaspersky, E. (2013). One in twenty is the sad truth. Retrieved from http://eugene. kaspersky.com/2013/03/25/one-in-twenty-is-the-sad-truth

Keizer, G. (2008). Crooks Can Make $\$ 5 \mathrm{M}$ a Year Shilling Fake Security Software. Computer World. Retrieved from http://www.computerworld.com/s/article/91 18778/Crooks_ can_make_5M_a_year_shilling_fake_security_software?pageNumber=1

Keizer, G. (2009). New Ransomware Holds Windows Files Hostage, Demands \$50. Computer World. Retrieved from http://www.computerworld.com/s/article/9130539/New_ ransomware_holds_Windows_files_hostage_demands_50 
Kervyn, N., Judd, C. M., \& Yzerbyt, V. Y. (2009). You want to appear competent? Be mean! You want to appear sociable? Be lazy! Group differentiation and the compensation effect. Fournal of Experimental Social Psychology, 45(2), 363-367. doi:10.1016/j. jesp.2008.08.006

Kirda, E., \& Kruegel, C. (2006). Protecting users against phishing attacks. The Computer Fournal, 49(5), 554-561. doi:10.1093/comjnl/bxh169

Klein, H. K., \& Myers, M. D. (1999). A set of principles for conducting and evaluating interpretive field studies in information systems. MIS Quarterly, 23(1), 67-94. doi: $10.2307 / 249410$

Kotadia, M. (2005). Mac community must wake up to security. ZDNet. Retrieved from http://www.zdnet.com/mac-community-must-wake-up-to-security-1139210762/

LaGrange, R. L., \& Ferraro, K. F. (1989). Assessing age and gender differences in perceived risk and fear of crime. Criminology, 27(4), 697-720. doi:10.1111/j.1745-9125.1989. tb01051.x

LaRose, R., \& Rifon, N. J. (2007). Promoting i-safety: Effects of privacy warnings and privacy seals on risk assessment and online privacy behavior. The fournal of Consumer Affairs, 41(1), 127-149. doi:10.1111/j.1745-6606.2006.00071.x

LaRose, R., Rifon, N., Liu, S., \& Lee, D. (2005). Online safety strategies: A content analysis and theoretical assessment. Paper presented at the 55th Annual Conference of the International Communication Association, New York, New York.

LaRose, R., Rifon, N. J., \& Enbody, R. (2008). Promoting personal responsibility for internet safety. Communications of the Association for Information Systems, 51(3), 71-76. doi:10.1145/1325555.1325569

LaRose, R., Rifon, N. J., \& Wirth, C. (2007). Online safety begins with you and me: Getting internet users to protect themselves. Paper presented at the 57th Annual Conference of the International Communication Association, San Francisco, California.

LaRose, R., Rifton, N., Liu, S., \& Lee, D. (2005). Understanding online safety behavior: A multivariate model. Paper presented at the 55th Annual Conference of the International Communication Association, New York, New York.

Lee, D., Larose, R., \& Rifon, N. (2008). Keeping our network safe: A model of online protection behaviour. Behaviour \& Information Technology, 27(5), 445-454. doi:10.1080/01449290600879344

Lee, J., \& Lee, Y. (2002). A holistic model of computer abuse within organizations. Information Management \& Computer Security, 10(2), 57-63. doi:10.1108/09685220210424104

Lee, M. K. O., \& Turban, E. (2001). A trust model for consumer internet shopping. International fournal of Electronic Commerce, 6(1), 75-91. 
Lemos, R. (2013). Cyber-Criminals May be switching to more aggressive scareware scams. eWeek. Retrieved from http://www.eweek.com/security/cyber-criminals-may-beswitching-to-more-aggressive-scareware-scams/

Leonard, L. N., Gronan, T. P., \& Krele, J. (2004). What influences IT ethical behavior intentions - planned behavior, reasoned action, perceived importance, or individual characteristics? Information \& Management, 42(1), 143-158. doi:doi:10.1016/j. im.2003.12.008

Leyden, J. (2007). Feeling left out? Get your PC infected today! Spoof malware campaign racks in the hits. The Register. Retrieved from http://www.theregister.co.uk/2007/05/17/ spoof_malware_campaign/

Liang, H., \& Xue, Y. (2009). Avoidance of information technology threats: A theoretical perspective. MIS Quarterly, 33(1), 71-90.

Liang, H., \& Xue, Y. (2010). Understanding security behaviors in personal computer usage: A threat avoidance perspective. Fournal of the Association for Information Systems, 11(7), 394-413. doi:10.1.1.170.5816

Lincoln, Y. S., \& Guba, E. G. (1986). But is it rigorous? Trustworthiness and authenticity in naturalistic evaluation. New Directions for Program Evaluation, 1986(30), 73-84. doi:10.1002/ev.142

Lineberry, S. (2007). The human element: The weakest link in information security. Fournal of Accountancy, 204(5), 44.

Lu, H. -P., Hsu, C. -L., \& Hsu, H. -Y. (2006). An empirical study of the effect of perceived risk upon intention to use online applications. Information Management \& Computer Security, 13(2), 106-120. doi:10.1108/09685220510589299

Marakas, G. M., Yi, M. Y., \& Johnson, R. D. (1998). The multilevel and multifaceted character of computer self-efficacy: Toward clarification of the construct and an integrative framework for research. Information Systems Research, 9(2), 126-163. doi:10.1287/isre.9.2.126

Markoff, J. (2009). Do we need a new Internet? New York Times, p. WK1. Retrieved from http:/ /www.nytimes.com/2009/02/15/weekinreview/15markoff.html

Maslow, A. H. (1970). Motivation and personality (2 ed.). New York: Harper \& Row.

Mays, N., \& Pope, G. (1995). Rigour and qualitative research. British Medical Fournal, 311, 109-112.

McDowell, M., \& Householder, A. (2004). US-CERT Gyber Security Tip ST04-001 -Why Is Cyber Security a Problem. Retrieved from http://www.us-cert.gov/cas/ tips/ST04-001.html 
McKnight, D. H., Choudhury, V., \& Kacmar, C. (2002). Developing and validating trust measures fore-commerce: An integrative typology. Information Systems Research, 15(3), 334-359.

Microsoft. (2006). How to prevent cross-site scripting security issues. Retrieved from http:// support.microsoft.com/kb/252985

Miles, M. B., \& Huberman, A. M. (1994). Qualitative data analysis: An expanded sourcebook. Thousand Oaks: SAGE Publications.

Mills, E. (2012). What the password leak means to you (FAQ). CNET. Retrieved from http://news.cnet.com/8301-1009_3-57449325-83/what-the-password-leaks-meanto-you-faq/

Myers, M. D., \& Newman, M. (2007). The qualitative interview in IS research: Examining the craft. Information and Organization, 17(1), 2-26. doi:10.1016/j.infoandorg.2006.11.001

Naraine, R. (2008). Clickjacking: Researchers raise alert for scary new cross-browser exploit. ZDNet. Retrieved from http://www.zdnet.com/blog/security/clickjackingresearchers-raise-alert-for-scary-new-cross-browser-exploit/1972

Neilsen Company. (2008). Two-thirds of online Kiwis now use internet banking. Retrieved from http://nz.nielsen.com/news/CFM_Feb08.shtml

NetBasics. (2008). Netsafe NetBasics. Retrieved from http://www.netsafe.org.nz

Neuwirth, K., Dunwoody, S., \& Griffin, R. J. (2000). Protection motivation and risk communication. Risk Analysis, 20(5), 721-733. doi:10.1111/0272-4332.205065

New Zealand Ministry of Social Development. (2010). Telephone and internet access in the home. The Social Report 2010. Retrieved from http://socialreport.msd.govt.nz/ social-connectedness/telephone-internet-access.htm

Ng, B. -Y., \& Rahim, M. A. (2005). A socio-behavioral study of home computer users' intention to practice security. Presented at the Ninth Pacific Asia Conference on Information Systems, Bangkok, Thailand, 234-247.

Ng, B. -Y., Kankanhalli, A., \& Xu, Y. (2008). Studying users' computer security behavior: A health belief perspective. Decision Support Systems, 46(4), 815-825. doi:10.1016/j. dss.2008.11.010

Onwuegbuzie, A. J., \& Leech, N. L. (2005). Taking the "Q" out of research: Teaching research methodology courses without the divide between quantitative and qualitative paradigms. Quality and Quantity, 39(3), 267-295. doi:10.1007/s11135-004-1670-0

Orlikowski, W. J., \& Baroudi, J.J. (1991). Studying information technology in organizations: Research approaches and assumptions. Information Systems Research, 2(1), 1-28. doi:10.1287/isre.2.1.1 
Panda Security. (2008). Quarterly report PandaLabs (April - June 2008). Retrieved from http://pandalabs.pandasecurity.com/blogs/images/PandaLabs/2008/07/07 / Quartely_Report_Q2_PandaLabs_2008.pdf

Panda Security. (2013). Nearly a third of all computers scanned around the world were infected, reports PandaLabs. Retrieved from http://press.pandasecurity.com/usa/ news/nearly-a-third-of-all-computers-scanned-around-the-world-in-2012-wereinfected-reports-pandalabs/

Perlroth, N. (2012, December 31). Outmaneuvered at their own game, antivirus makers struggle to adapt. The New York Times, p. B1. Retrieved from http://www.nytimes. com/2013/01/01/technology/antivirus-makers-work-on-software-to-catchmalware-more-effectively.html

Perlroth, N., \& Sanger, D. E. (2013, July 13). Nations buying as hackers sell knowledge of software flaws. The New York Times, p. A1. Retrieved from http://www.nytimes. com/2013/07/14/world/europe/nations-buying-as-hackers-sell-computer-flaws. html

Petersen, R., \& Worona, S. (2006). Privacy \& security: An overview. EDUCAUSE, 41(5), 16-17.

Pew Research Center. (2013). Millennials: A portrait of generation next. Retrieved from http://www.pewresearch.org/millennials/teen-internet-use-graphic/

Plotnikoff, R. C., \& Higginbotham, N. (2002). Protection motivation theory and exercise behaviour change for the prevention of coronary heart disease in a high-risk, Australian representative community sample of adults. Psychology, Health \& Medicine, 7(1), 87-98.

Protalinski, E. (2013). Retail e-commerce hit $\$ 186.2 \mathrm{~b}$ in 2012 thanks to $15 \%$ growth, the strongest since before the recession - the next web. ComScore. Retrieved from http:// thenextweb.com/insider/2013/02/07/comscore-retail-e-commerce-hit-186-2b-in2012-thanks-to-15-growth-the-strongest-since-before-the-recession/

PwC, \& Frost \& Sullivan. (2012). Australia and New Zealand online shopping market and digital insights : July 2012. Retrieved from http://www.pwc.com.au/industry/retailconsumer/assets/Digital-Media-Online-Shopping-Jul12.pdf

Rains, T. (2013). Latest security intelligence report shows 24 percent of PCs are unprotected. Retrieved from http://blogs.technet.com/b/microsoft_blog/archive/2013/04/17/ latest-security-intelligence-report-shows-too-many-pcs-lack-antivirus-protection. aspx 
Rhee, H. -S., Kim, C., \& Ryu, Y. U. (2009). Self-efficacy in information security: Its influence on end users' information security practices behavior. Computers \& Security, 28, 816-826. doi:10.1016/j.cose.2009.05.008

Rhee, H. -S., Ryu, Y., \& Kim, C. -T. (2005). I am fine but you are not: Optimistic bias and illusion of control on information security. Presented at the Twenty-Sixth International Conference on Information Systems, Las Vegas, Nevada.

Rhodes, R. E., \& Plotnikoff, R. C. (2005). Can current physical activity act as a reasonable proxy measure of future physical activity? Evaluating cross-sectional and passive prospective designs with the use of social conition models. Preventive Medicine, 40(5), 547-555. doi:10.1016/j.ypmed.2004.07.016

Rifon, N. J., Quilliam, E. T., \& LaRose, R. (2005). Consumer perceptions of online safety. Presented at the 55th Annual Conference of the International Communications Association, New York, New York.

Rose, J. P., Endo, Y., Windschitl, P. D., \& Suls, J. (2008). Cultural differences in unrealistic optimism and pessimism: The role of egocentrism and direct versus indirect comparison measures. Personality \& Social Psychology Bulletin, 34(9), 1236-48. doi:10.1177/0146167208319764

Rosenstock, I. M., Strecher, V.J., \& Becker, M. H. (1988). Social learning theory and the health belief model. Health Education \& Behavior, 15, 175. doi:10.1177/109019818801500203

Ruiter, R. A. C., Abraham, G., \& Kok, G. (2001). Scary warnings and rational precautions: A review of the psychology of fear appeals. Psychology \& Health, 16(6), 613-630. doi:10.1080/08870440108405863

Sasse, M. A., Brostoff, S., \& Weirich, D. (2004). Transforming the 'weakest link' - a human/ computer interaction approach to usable and effective security. BT Technology fournal, 19(3), 122-131. doi:10.1023/A:1011902718709

Schwartz, M. J. (2013a). Evernote breach: 7 security lessons. InformationWeek. Retrieved from http://www.informationweek.com/security/attacks/evernote-breach-7security-lessons/240149911

Schwartz, M. J. (2013b). Microsoft, FBI trumpet citadel botnet takedowns. InformationWeek. Retrieved from http://www.informationweek.com/security/attacks/microsoft-fbitrumpet-citadel-botnet-tak/240156171

Shachtman, N. (2008). Under Worm Assault, Military Bans Disks, USB Drives. Wired. Retrieved from http://www.wired.com/dangerroom/2008/11/army-bans-usb-d/

Sheehan, K. B. (2002). Toward a typology of internet users and online privacy concerns. The Information Society, 18(1), 21-32. doi:10.1080/01972240252818207

Singh, K. (2007). Qualitative social research methods: Methods and issues. SAGE. 
Skibell, R. (2002). The myth of the computer hacker. Information, Communication E̊ Society, 5(3), 336-356. doi:10.1080/13691180210159292

Smith, A. D. (2004). E-security issues and policy development in an information-sharing and networked environment. Aslib Proceedings: New Information Perspectives, 56(5), 272285. doi:10.1108/00012530410560878

Sophos. (2008). Security threat report // Q1 08. Retrieved from www.sophos.com/sophos/ docs/eng/.../sophos-threat-report-Q108.pdf

Spitzner, L. (2003). Honeypots: Definitions and value of honeypots. Retrieved from http:/ / www.tracking-hackers.com/papers/honeypots.html

Staniford, S., Paxson, V., \& Weaver, N. (2002). How to Own the internet in your spare time. Proceedings of the 11 th USENIX Security Symposium, San Francisco, California. 149-167.

Stanton, J. M., Stam, K. R., Mastrangelo, P., \& Jolton, J. (2005). Analysis of end user security behaviors. Computers \& Security, 24(2), 124-133. doi:10.1016/j.cose.2004.07.001

Sund, C. (2007). Towards an international road-map for cybersecurity. Online Information Reviere, 31(5), 566-582. doi:10.1108/14684520710832306

Swire, P. (2004). A model for when disclosure helps security: What is different about computer and network security? Telecommunications and High Technology Law, 2, 2-38.

Symantec. (2013). Security response: Spam. Retrieved from http://www.symantec.com/ security_response/landing/spam/

Symantec Corporation. (2008). Symantec global internet security threat report: Trends for July-December 07. Retrieved from http://eval.symantec.com/mktginfo/enterprise/ white_papers/b-whitepaper_internet_security_threat_report_xiii_04-2008.en-us. pdf

Symantec Corporation. (2009). Symantec global internet security threat report: Trends for 2008. Retrieved from http://eval.symantec.com/mktginfo/enterprise/white_ papers/b-whitepaper_internet_security_threat_report_xiv_04-2009.en-us.pdf

Symantec Intelligence. (2013). Symantec intelligence report: February 2013. Retrieved from http://www.symantec.com/theme.jsp?themeid=state_of_spam

Talib, S., Clarke, N. L., \& Furnell, S. M. (2010). An analysis of information security awareness within home and work environments. International Conference on Availability, Reliability, and Security, 196-203. doi:10.1109/ARES.2010.27

Thompson, S. T. C. (2006). Helping the hacker? Library information, security, and social engineering. Information Technology and Libraries, 25(4), 222-225. 
Trend Micro. (2008). 2007 threat report 2008 threat and technology forecast. Retrieved from http://us.trendmicro.com/imperia/md/content/us/pdf/threats/securitylibrary/ tre_threat_report.pdf

Tsohou, A., Karyda, M., Kokolakis, S., \& Kiountouzis, E. (2006). Formulating information systems risk management strategies through cultural theory. Information Management E Computer Security, 14(3), 198-217. doi:10.1108/09685220610670378

Tsukayama, H. (2012). Yahoo password breach reveals 400k passwords. The Washington Post. Retrieved from http://www.washingtonpost.com/blogs/post-tech/post/yahoopassword-breach-reveals-400k-passwords-thecircuit/2012/07/12/gJQAiz1RfW_ blog.html

U.S. Cyber Security Information Act. (2000). HR 4246 .

United States Computer Emergency Readiness Team. (2001). Home Network Security Email Spoofing. Retrieved from http://search.us-cert.gov/

United States Computer Emergency Readiness Team. (2008). Cyber Security Tips. Retrieved from http://www.us-cert.gov/cas/tips/ST04-001.html

United States Department of Homeland Security. (2003). The national strategy to secure cyberspace. The national strategy to secure cyberspace. United States Department of Homeland Security. Retrieved from http://www.dhs.gov/national-strategy-securecyberspace

Verhagen, T., Meents, S., \& Tan, Y. -H. (2006). Perceived risk and trust associated with purchasing at electronic marketplaces. European Fournal of Information Systems, 15(6), 542-555. doi:10.1057/palgrave.ejis.3000644

von Solms, R., \& van Niekerk, J. (2013). From information security to cyber security. Computers \& Security, 97-102. doi:http://dx.doi.org/10.1016/j.cose.2013.04.004

Wang, J., Xiao, N., \& Rao, H. R. (2010). Drivers of information security search behavior: An investigation of network attacks and vulnerability disclosures. ACM Transactions on Management Information Systems, 1(1), 3. doi:10.1145/1877725.1877728

Warr, M., \& Ellison, G. G. (2000). Rethinking social reactions to crime: Personal and altruistic fear in family households. American Fournal of Sociology, 106(3), 551-578.

Webb, T. (2007). Criminals 'may Overwhelm the Web'. BBC. Retrieved from http://news. bbc.co.uk/2/hi/business/6298641.stm

Weirich, D., \& Sasse, M. (2001). Persuasive password security. Presented at the Conference on Human Factors in Computing Systems, Seattle, Washington, 139-140.

Wilbanks, L. (2007). Cybersecurity: Welcome to my world. IT Professional, 9(2), 61. 
Willison, R. (2006). Understanding the perpetration of employee computer crime in the organisational context. Information and Organization, 16(4), 304-324. doi:10.1016/j. infoandorg.2006.08.001

Wilson, T. (2009). Scareware Morphs Into Ransomware. Dark Reading. Retrieved from http://www.darkreading.com/security/attacks-breaches/216300413/index.html

Wilson, T. (2011). Report: Botnet victim population grew more than 600 percent in 2010. Dark Reading. Retrieved from http://www.darkreading.com/vulnerabilitymanagement/167901026/security/attacks-breaches/229219325/report-botnetvictim-population-grew-more-than-600-percent-in-2010.html

Wilson, T. (2013). Consumer reports: 58 million U.S. PGs infected with malware. Dark Reading. Retrieved from http://www.darkreading.com/privacy/consumer-reports58-million-us-pcs-infec/240154081

Wirth, G. B., Rifon, N. J., LaRose, R. J., \& Lewis, M. L. (2007). Promoting teenage online safety with an $i$-safety intervention: Enhancing self-efficacy and protective behaviors. Proceedings of the 58th Annual Conference of the International Communication Asociation, Québec, Canada.

Witte, K., \& Allen, M. (2000). A meta-analysis of fear appeals: Implications for effective public health campaigns. Health Education \& Behavior, 27(5), 591-615. doi:10.1177/109019810002700506

Woon, I., Tan, G. -W., \& Low, R. (2005). A protection motivation theory approach to home wireless security. Presented at the Twenty-Sixth International Conference on Information Systems, Las Vegas, Nevada, 367-380.

Workman, M. (2007). Gaining access with social engineering: An empirical study of the threat. Information Systems Security, 16(6), 315-331. doi:10.1080/10658980701788165

ZDNet. (2008). Cybersecurity: Definition and Additional Resources From ZDNet. Retrieved from ZDNet.com

Zikmund, W. G. (1987). Business research methods. Dryden Press. 
Braun 


\section{Appendices}

Appendix 1 Definitions..........................................................283

Appendix 2 Overview of literature .........................................28

Appendix 3 Overview of top level codes ..............................287

Appendix 4 List of codes and definitions ...............................289

Appendix 5 Mind map of initial relationships between

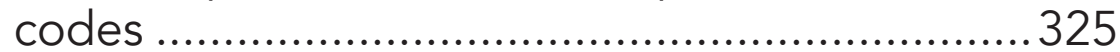

Appendix 6 Information sheet \& consent form ......................327

Appendix 7 Explanation of ranking used in the coding process...........................................................333 
Braun 


\section{Appendix $1 \quad$ Definitions}

\section{A}

AJAX \& JavaScript Website tools that can be use to speed up websites, but that are also Vulnerabilities vulnerable to attacks by malicious users (TrendMicro, 2008)

\section{B}

Behavioural Safeguards Steps that an individual takes to protect their cybersecurity. These steps are typically associated with the things that individuals do rather than whether or not they have protective software installed on their computer.

Cross-Scripting A vulnerability that allows unverified scripts to be run on legitimate sites (Microsoft, 2008)

Cybersecurity The protection of data from unauthorised use and from unwanted destruction by Internet threats (including, but not limited to spam, phishing, malware, and other web based threats)

Cybersecurity Breach When a cybersecurity threat affects an individual's computer leading to data, personal, or other consequences which require the individual to take steps to remedy.

Cybersecurity Threat Malicious software and the actions of malicious users which can lead to cybersecurity breaches. A cybersecurity threat does not have to affect a person's computer to be a cybersecurity threat.

\section{$\mathbf{H}$}

Honeypot An "information system resource whose value lies in the unauthorised or illicit use of that resource" Honeypots can be used to "defend against such attacks by slowing their scanning down, potentially even stopping them" (Spitzner, 2003).

iFrames Invisible hidden elements on webpages that allow malicious users' to compromise legitimate websites (Trend Micro, 2008)

\section{M}

Malicious User An individual who writes, propagates, or is otherwise responsible for cybersecurity threats. Despite the term "hackers" often being used to refer to malicious users, it should be noted that a hackers do not always act maliciously. Therefore, the term malicious users is used.

Patch Software and operating system updates in order to fix problems, update functionality, or close security vulnerabilities in the system. Failure to ensure that a piece of software is patched can result in vulnerabilities that can be exploited by malicious users.

Phishing A social engineering technique used to gain sensitive information from the targeted person, often through spoofing. Spoofing is when a malicious user impersonates a legitimate website or person to gain the recipients confidence (United States Computer Emergency Readiness Team, 2001). 
Protective Behaviours The steps that individuals take to protect their security online. This can involve both behavioural safeguards and the use of protective software.

Protective Software Software such as antivirus software, firewalls, and anti-spyware software that is used to identify and prevent cybersecurity breaches. Also referred to as protective technologies.

\section{$\mathbf{R}$}

Ransomware A piece of malware that encrypts files on a user's computer and then promotes another piece of software (typically malicious) to restore the files for a fee, effectively holding the user's files ransom. This not only provides malicious users with the opportunity to install further malware on a user's computer, but also to get their credit card information if they pay the fee to restore their files.

\section{$\mathbf{S}$}

Spam Unsolicited emails that usually attempt to see something or to offer a disproportionately high benefit to the recipient (Australian Communications and Media Authority, 2007).

\section{$\mathbf{T}$}

Trojan Horse A program that appears to do one thing, but instead does something else, usually harmful to the computer in some way (Smith, 2004)

\section{$\mathbf{U}$}

User-Related Risks Risks associated with unsafe cybersecurity behaviours, such as sharing passwords, the use of weak passwords, and opening emails without first scanning them for viruses. These risks depend on the user's behaviour rather than a technological weakness that can be exploited (Aytes \& Connolly, 2004).

\section{V}

Virus A programs that replicates itself on users' computers similar to how a human virus replicates in a person's body (Australian Communications and Media Authority, 2007).

\section{$\mathbf{W}$}

Worm Malicious software that travels across computer networks. Unlike viruses and trojan horses, they do not require another program to launch them (Smith, 2004; Staniford et al., 2002).

\section{$\mathbf{Z}$}

Zero Day Bug Threats "that once discovered, "zero days" exist for the user of the computer system to fix them before hackers can take advantage of the vulnerability. A "zero-day" exploit occurs when hackers or governments strike by using the flaw before anyone else knows it exists" (Perlroth \& Sanger, 2013) 


\section{Appendix 2 Overview of literature}

Table A-1 Overview of literature based on the researched components

\begin{tabular}{|c|c|c|c|c|}
\hline Author (Year) & Actor & Objective & Method & Duration \\
\hline Adams (1999) & $\mathrm{O}$ & $\mathrm{S}$ & So & $\mathrm{G}$ \\
\hline Ahmed (2007) & - & $\mathrm{S}$ & $\mathrm{T}$ & - \\
\hline Al-Khouri (2007) & - & $\mathrm{S}$ & $\mathrm{T}$ & - \\
\hline Albrechtsen (2007) & $\mathrm{O}$ & $\mathrm{S}$ & So & $\mathrm{G}$ \\
\hline Anderson (2006) & $\mathrm{H}$ & $\mathrm{S}$ & So & $\mathrm{C}$ \\
\hline Aytes (2004) & $\mathrm{H}$ & $\mathrm{S}$ & So & $\mathrm{G}$ \\
\hline Baskerville (2003) & $\mathrm{O}$ & $\mathrm{S}$ & $\mathrm{T}$ & - \\
\hline Boukerche (2004) & - & $\mathrm{S}$ & $\mathrm{T}$ & - \\
\hline Chan (2005) & $\mathrm{O}$ & $\mathrm{S}$ & So & $\mathrm{C}$ \\
\hline Charoen (2008) & $\mathrm{O}$ & $\mathrm{S}$ & $\mathrm{T}$ & $\mathrm{G}$ \\
\hline Chou (1999) & $\mathrm{O}$ & $\mathrm{S}$ & So / T & $\mathrm{G} / \mathrm{A}$ \\
\hline Clayton (2008 & - & $\mathrm{S}$ & $\mathrm{T}$ & - \\
\hline Culnan (2008) & $\mathrm{H} / \mathrm{O}$ & $\mathrm{S}$ & So & $\mathrm{C}$ \\
\hline Dentu (2007) & - & $\mathrm{S}$ & $\mathrm{T}$ & - \\
\hline Dinev (2006) & $\mathrm{H}$ & $\mathrm{P}$ & So & - \\
\hline Dinev (2007) & $\mathrm{H}$ & $\mathrm{S}$ & $\mathrm{T}$ & $\mathrm{A}$ \\
\hline Dodge (2007) & $\mathrm{H}$ & $\mathrm{S}$ & $\mathrm{T}$ & $\mathrm{C}$ \\
\hline Drennan (2006) & $\mathrm{H}$ & $\mathrm{P}$ & So & $\mathrm{G}$ \\
\hline Felchais (2003) & - & $\mathrm{S}$ & $\mathrm{T}$ & - \\
\hline Fucs (2007) & - & $\mathrm{S}$ & $\mathrm{T}$ & - \\
\hline Furnell (2007) & $\mathrm{H}$ & $\mathrm{S}$ & So / T & $\mathrm{G} / \mathrm{A}$ \\
\hline Garrison (2008) & $\mathrm{O}$ & $\mathrm{S}$ & $\mathrm{T}$ & $\mathrm{G}$ \\
\hline Good (2006) & $\mathrm{H}$ & $\mathrm{S}$ & $\mathrm{T}$ & $\mathrm{A}$ \\
\hline Han (2002) & $\mathrm{H}$ & $\mathrm{P}$ & So & - \\
\hline Hazari (2005) & $\mathrm{O}$ & $\mathrm{S}$ & $\mathrm{T}$ & $\mathrm{A}$ \\
\hline Hofmeyr (2004) & - & $\mathrm{S}$ & $\mathrm{T}$ & - \\
\hline Kirda (2006) & $\mathrm{H}$ & $\mathrm{S}$ & $\mathrm{T}$ & - \\
\hline Knapp (2007 & $\mathrm{O}$ & $\mathrm{S}$ & So & $\mathrm{G}$ \\
\hline Kruck (2006) & $\mathrm{H}$ & $\mathrm{S}$ & $\mathrm{T}$ & $\mathrm{C} / \mathrm{A}$ \\
\hline Lallmahamood (2007) & $\mathrm{H}$ & $\mathrm{S} / \mathrm{P}$ & So & $\mathrm{A}$ \\
\hline LaRose (2005) & $\mathrm{H}$ & $\mathrm{S}$ & - & - \\
\hline LaRose (2005) & $\mathrm{H}$ & $\mathrm{S}$ & So & $\mathrm{G}$ \\
\hline LaRose (2007) & $\mathrm{H}$ & $\mathrm{S}$ & So & $\mathrm{C}$ \\
\hline LaRose (2007) & $\mathrm{H}$ & $\mathrm{P}$ & So & $\mathrm{G}$ \\
\hline Key & $\begin{array}{l}\text { H - Home } \\
\text { User } \\
\text { O - Org User }\end{array}$ & $\begin{array}{l}\text { P - Privacy } \\
\text { S - Security }\end{array}$ & $\begin{array}{l}\text { So - Social } \\
\mathrm{T} \text { - } \\
\text { Technical }\end{array}$ & $\begin{array}{l}\text { C - Ongoing } \\
\text { A - One- } \\
\text { Time }\end{array}$ \\
\hline
\end{tabular}




\begin{tabular}{|c|c|c|c|c|}
\hline Author (Year) & Actor & Objective & Method & Duration \\
\hline LaRose (2008) & $\mathrm{H}$ & $\mathrm{S}$ & So & $\mathrm{G}$ \\
\hline Lee (2002) & $\mathrm{O}$ & $\mathrm{S}$ & So & $\mathrm{G}$ \\
\hline Leonard (2004) & $\mathrm{I}$ & $\mathrm{S}$ & So & $\mathrm{G}$ \\
\hline $\mathrm{Lu}(2006)$ & $\mathrm{H}$ & $\mathrm{S} / \mathrm{P}$ & So & $\mathrm{C}$ \\
\hline Milne (2004) & $\mathrm{H}$ & $\mathrm{P}$ & So & - \\
\hline Milne (2004) & $\mathrm{H}$ & $\mathrm{P}$ & So & $\mathrm{C}$ \\
\hline $\mathrm{Ng}(2005)$ & $\mathrm{H}$ & $\mathrm{S}$ & So & $\mathrm{G}$ \\
\hline $\mathrm{Ng}(2008)$ & $\mathrm{O}$ & $\mathrm{S}$ & So & $\mathrm{G}$ \\
\hline Pahnila (2007) & $\mathrm{H}$ & $\mathrm{S}$ & So & $\mathrm{G}$ \\
\hline Provos (2007) & - & $\mathrm{S}$ & $\mathrm{T}$ & - \\
\hline Rice (2007) & - & $\mathrm{S}$ & $\mathrm{T}$ & - \\
\hline Rifon (2005) & $\mathrm{H}$ & $\mathrm{S}$ & So & $\mathrm{G} / \mathrm{A}$ \\
\hline Ryu (2005) & $\mathrm{O}$ & $\mathrm{S}$ & So & $\mathrm{C}$ \\
\hline Salisbury (2001) & $\mathrm{H}$ & $\mathrm{S}$ & $\mathrm{T}$ & $\mathrm{A}$ \\
\hline Sasse (2004) & $\mathrm{O}$ & $\mathrm{S}$ & So & $\mathrm{C}$ \\
\hline Smith (2004) & $\mathrm{O}$ & $\mathrm{S}$ & So / T & $\mathrm{C} / \mathrm{A}$ \\
\hline Somayaji (2007) & - & $\mathrm{S}$ & $\mathrm{T}$ & - \\
\hline Staniford (2002) & - & $\mathrm{S}$ & $\mathrm{T}$ & - \\
\hline Stanton (2005) & $\mathrm{O}$ & $\mathrm{S}$ & So & $\mathrm{G}$ \\
\hline Straub (1990) & $\mathrm{O}$ & $\mathrm{S}$ & $\mathrm{T}$ & - \\
\hline Weaver (2003) & - & $\mathrm{S}$ & $\mathrm{T}$ & - \\
\hline Weirich (2001) & - & $\mathrm{S}$ & So & $\mathrm{G}$ \\
\hline Wirth (2007) & $\mathrm{H}$ & $\mathrm{S}$ & So & $\mathrm{C}$ \\
\hline Woon (2005) & $\mathrm{H}$ & $\mathrm{S}$ & So & $\mathrm{A}$ \\
\hline Workman (2007) & $\mathrm{O}$ & $\mathrm{S}$ & So & - \\
\hline Youn $(2005)$ & $\mathrm{H}$ & $\mathrm{P}$ & So & $\mathrm{G}$ \\
\hline Zviran (1999) & $\mathrm{O}$ & $\mathrm{S}$ & $\mathrm{T}$ & - \\
\hline Key & $\begin{array}{l}\text { H - Home } \\
\text { User } \\
\text { O - Org User }\end{array}$ & $\begin{array}{l}\text { P - Privacy } \\
\text { S - Security }\end{array}$ & $\begin{array}{l}\text { So - Social } \\
\mathrm{T} \text { - } \\
\text { Technical }\end{array}$ & $\begin{array}{l}\text { C - Ongoing } \\
\text { A - One- } \\
\text { Time }\end{array}$ \\
\hline
\end{tabular}




\section{Appendix 3 Overview of top level codes}

Table A-2 Top Level Codes from Focus Groups and Interviews

\begin{tabular}{|l|l|l|}
\hline Age & InformationSources & Skill \\
\hline Analogies & Knowledge & Threats \\
\hline AverageUsers & Ego Protection & Trust \\
\hline Awareness & MaliciousUsers & UserMotivations \\
\hline Barriers & Notifications & Value \\
\hline Beliefs & PerceivedSeverity & WorkImpact \\
\hline Benefits & PerceivedSusceptibility & \\
\hline Confidence & PriorExperience & \\
\hline Consequences & Privacy & \\
\hline CurrentBehaviours & Reliance & \\
\hline Definitions & Responsibility & \\
\hline Feelings & Safeguards & \\
\hline FuturePredictions & ServicesUses & \\
\hline Hardware & Sharing \\
\hline & \multicolumn{2}{|}{} \\
\hline
\end{tabular}


Braun 


\section{Appendix $4 \quad$ List of Codes and Definitions}

\begin{tabular}{|c|c|c|c|}
\hline $\begin{array}{c}\text { Level } \\
1\end{array}$ & \begin{tabular}{|c|} 
Level \\
2 \\
\end{tabular} & $\begin{array}{c}\text { Level } \\
3\end{array}$ & Definition \\
\hline \multicolumn{4}{|l|}{ Age } \\
\hline & \multicolumn{2}{|c|}{ Age_Concern } & $\begin{array}{l}\text { Perception that older people are more } \\
\text { concerned about potential security } \\
\text { breaches than younger people. }\end{array}$ \\
\hline & \multicolumn{2}{|c|}{ Age_Fear } & $\begin{array}{l}\text { Perception that older internet users are } \\
\text { more afraid of potential cybersecurity } \\
\text { threats. }\end{array}$ \\
\hline & \multicolumn{2}{|c|}{ Age_Knowledge } & $\begin{array}{l}\text { Perception that younger users are more } \\
\text { knowledgeable about potential internet } \\
\text { security threats and how to deal with } \\
\text { them. }\end{array}$ \\
\hline & \multicolumn{2}{|c|}{ Age_Usage } & $\begin{array}{l}\text { Perception that age influences the usage } \\
\text { of computers or associated online } \\
\text { services. }\end{array}$ \\
\hline \multicolumn{4}{|c|}{ Analogies } \\
\hline & \multicolumn{2}{|c|}{ Analogy_Consequences } & $\begin{array}{l}\text { Analogy relating to the consequences of } \\
\text { an attack. }\end{array}$ \\
\hline & \multicolumn{2}{|c|}{ Analogy_Safeguards } & $\begin{array}{l}\text { Analogy relating to cybersecurity } \\
\text { safeguards and how they work. }\end{array}$ \\
\hline & \multicolumn{2}{|c|}{ Analogy_Threats } & $\begin{array}{l}\text { Analogy relating to what a cybersecurity } \\
\text { threat is and how it works. }\end{array}$ \\
\hline \multicolumn{4}{|c|}{ AverageUsers } \\
\hline & \multicolumn{2}{|c|}{ AvgUser_ProtectionAssumed } & $\begin{array}{l}\text { Belief that the "normal" person believes } \\
\text { that their computer includes a certain } \\
\text { level of protection from the time that } \\
\text { it is purchased and no further action is } \\
\text { required. }\end{array}$ \\
\hline & \multicolumn{2}{|c|}{ AvgUser_CannotAffordSecurity } & $\begin{array}{l}\text { "Perception that the "normal" user } \\
\text { cannot afford what is considered by } \\
\text { respondents to be "'good"'" security." }\end{array}$ \\
\hline & \multicolumn{2}{|c|}{ AvgUser_ComplacentRisk } & $\begin{array}{l}\text { Perception that the "normal" user is } \\
\text { satisfied with the level of risk that they } \\
\text { are taking. }\end{array}$ \\
\hline & \multicolumn{2}{|c|}{ AvgUser_NotProactive } & $\begin{array}{l}\text { Belief that the "normal" user requires } \\
\text { some sort of catalyst event to start } \\
\text { safeguarding themselves. Otherwise, the } \\
\text { average user does not act in a protective } \\
\text { manner. }\end{array}$ \\
\hline
\end{tabular}




\begin{tabular}{|c|c|c|c|c|}
\hline \multirow[t]{2}{*}{$\begin{array}{c}\text { Level } \\
1\end{array}$} & $\begin{array}{c}\text { Level } \\
2\end{array}$ & $\begin{array}{c}\text { Level } \\
3\end{array}$ & Level 4 & Definition \\
\hline & \multicolumn{3}{|c|}{ AvgUser_DoesNotKnowBetter } & $\begin{array}{l}\text { Belief that the "normal" user does not } \\
\text { have enough knowledge to be aware } \\
\text { of the threats that are online or the } \\
\text { safeguards that can be taken to protect } \\
\text { themselves from these threats. }\end{array}$ \\
\hline & \multicolumn{3}{|c|}{ AvgUser_NoThreatAttention } & $\begin{array}{l}\text { Perception that the "normal" user does } \\
\text { not pay attention to the potential threats } \\
\text { online. }\end{array}$ \\
\hline & \multicolumn{3}{|c|}{ AvgUser_NoExtraPrecautions } & $\begin{array}{l}\text { Perception that the "normal" user does } \\
\text { not take any additional steps to protect } \\
\text { themselves from the potential online } \\
\text { threats. }\end{array}$ \\
\hline & \multicolumn{3}{|c|}{ AvgUser_LacksUnderstanding } & $\begin{array}{l}\text { Perception that the "normal" person is } \\
\text { uninformed / not knowledgeable about } \\
\text { how internet security threats work or } \\
\text { what the threats are. }\end{array}$ \\
\hline & \multicolumn{3}{|c|}{ AvgUser_KnowsBetter } & $\begin{array}{l}\text { Perception that the "normal" user } \\
\text { is better informed about threats and } \\
\text { sources of threats and therefore is } \\
\text { generally able to successfully avoid } \\
\text { them. }\end{array}$ \\
\hline & \multicolumn{3}{|c|}{ AvgUser_KnowsGenPrecautions } & $\begin{array}{l}\text { Perception that the "normal" user knows } \\
\text { enough about threats and safeguards to } \\
\text { take the necessary basic precautions and } \\
\text { that they do so. }\end{array}$ \\
\hline & \multicolumn{3}{|c|}{ AvgUser_NotConcerned } & $\begin{array}{l}\text { Perception that the "normal" user is not } \\
\text { concerned with internet threats. }\end{array}$ \\
\hline & \multicolumn{3}{|c|}{ AvgUser_UnknownAttachments } & $\begin{array}{l}\text { Perception that the "normal" user is } \\
\text { being adventurous but not necessarily } \\
\text { taking a risk when they open an email } \\
\text { from an unknown source. }\end{array}$ \\
\hline & \multicolumn{3}{|c|}{ AvgUser_OpensTooManyEmails } & $\begin{array}{l}\text { Perception that the "normal" user opens } \\
\text { too many emails (an identified threat } \\
\text { source) }\end{array}$ \\
\hline & \multicolumn{3}{|c|}{ AvgUser_VisitsTooManySites } & $\begin{array}{l}\text { "Perception that the "normal" user visits } \\
\text { too many websites (an identified threat } \\
\text { source), or too many "'unknown"" } \\
\text { websites." }\end{array}$ \\
\hline & \multicolumn{3}{|c|}{ AvgUser_ProtectFamily } & $\begin{array}{l}\text { Perception that the reason that the } \\
\text { "normal" user takes steps to protect } \\
\text { their internet security is in order to } \\
\text { protect their family. }\end{array}$ \\
\hline
\end{tabular}




\begin{tabular}{|c|c|c|c|c|}
\hline $\begin{array}{c}\text { Level } \\
1\end{array}$ & $\begin{array}{c}\text { Level } \\
2\end{array}$ & $\begin{array}{c}\text { Level } \\
3\end{array}$ & Level 4 & Definition \\
\hline \multicolumn{5}{|c|}{ Awareness } \\
\hline & \multicolumn{3}{|c|}{ Awareness_Safeguards } & $\begin{array}{l}\text { Perceptions of users about the awareness } \\
\text { of the "normal" user and themselves in } \\
\text { terms of safeguards. }\end{array}$ \\
\hline & & \multicolumn{2}{|c|}{ AvgUser_SafeguardAwareness } & $\begin{array}{l}\text { Perceptions of about how aware the } \\
\text { "normal" user is of the safeguards that } \\
\text { they can take to protect their computer. }\end{array}$ \\
\hline & & & AvgUserSafeguard_Low & $\begin{array}{l}\text { Perception that the "normal" person has } \\
\text { a low level of safeguard awareness. }\end{array}$ \\
\hline & & & AvgUserSafeguard_Mod & $\begin{array}{l}\text { Perception that the "normal" person has } \\
\text { a moderate level of safeguard awareness. }\end{array}$ \\
\hline & & & AvgUserSafeguard_High & $\begin{array}{l}\text { Perception that the "normal" person has } \\
\text { a high level of safeguard awareness. }\end{array}$ \\
\hline & & \multicolumn{2}{|c|}{ Respond_SafeguardAwareness } & $\begin{array}{l}\text { The self-rated perceptions of how aware } \\
\text { respondents are of the safeguards that } \\
\text { can be taken to protect their computers. }\end{array}$ \\
\hline & & & RespondSafeguard_Low & $\begin{array}{l}\text { The respondent indicated a low level of } \\
\text { safeguard awareness. }\end{array}$ \\
\hline & & & RespondSafeguard_Mod & $\begin{array}{l}\text { The respondent indicated a moderate } \\
\text { level of safeguard awareness. }\end{array}$ \\
\hline & & & RespondSafeguard_High & $\begin{array}{l}\text { The respondent indicated a high level of } \\
\text { safeguard awareness. }\end{array}$ \\
\hline & \multicolumn{3}{|c|}{ Awareness_Threats } & $\begin{array}{l}\text { Perceptions of respondents about the } \\
\text { awareness of the "normal" user and } \\
\text { themselves in terms of cybersecurity } \\
\text { threats. }\end{array}$ \\
\hline & & \multicolumn{2}{|c|}{ AvgUser_ThreatAwareness } & $\begin{array}{l}\text { Perceptions of about how aware the } \\
\text { "normal" user is of cybersecurity } \\
\text { threats. }\end{array}$ \\
\hline & & & AvgUserThreat_Low & $\begin{array}{l}\text { Perception that the "normal" person has } \\
\text { a low level of threat awareness. }\end{array}$ \\
\hline & & & AvgUserThreat_Mod & $\begin{array}{l}\text { Perception that the "normal" person has } \\
\text { a moderate level of threat awareness. }\end{array}$ \\
\hline & & & AvgUserThreat_High & $\begin{array}{l}\text { Perception that the "normal" person has } \\
\text { a high level of threat awareness. }\end{array}$ \\
\hline & & \multicolumn{2}{|c|}{ Respond_ThreatAwareness } & $\begin{array}{l}\text { The self-rated perceptions of how aware } \\
\text { respondents are of cybersecurity threats. }\end{array}$ \\
\hline & & & RespondThreat_Low & $\begin{array}{l}\text { The respondent indicated a low level of } \\
\text { threat awareness. }\end{array}$ \\
\hline & & & RespondThreat_Mod & $\begin{array}{l}\text { The respondent indicated a moderate } \\
\text { level of threat awareness. }\end{array}$ \\
\hline & & & RespondThreat_High & $\begin{array}{l}\text { The respondent indicated a high level of } \\
\text { threat awareness. }\end{array}$ \\
\hline
\end{tabular}




\begin{tabular}{|c|c|c|c|c|}
\hline $\begin{array}{c}\text { Level } \\
1\end{array}$ & $\begin{array}{c}\text { Level } \\
2\end{array}$ & $\begin{array}{c}\text { Level } \\
3\end{array}$ & Level 4 & Definition \\
\hline & & & RespondThreat_FBVirus & $\begin{array}{l}\text { The awareness that participants had } \\
\text { about a news article on a Facebook virus } \\
\text { that was presented to them during a } \\
\text { focus group exercise. }\end{array}$ \\
\hline \multicolumn{5}{|c|}{ Barriers } \\
\hline & \multicolumn{3}{|c|}{ Barrier_Impact } & $\begin{array}{l}\text { The impact that the barriers have on } \\
\text { respondents actually taking steps to } \\
\text { protect themselves. This is a self-rated } \\
\text { measure given by participants. }\end{array}$ \\
\hline & & \multicolumn{2}{|c|}{ BarrierImpact_Low } & $\begin{array}{l}\text { The respondent indicated they are } \\
\text { minimally affected by barriers to } \\
\text { engaging in cybersecurity behaviours. }\end{array}$ \\
\hline & & \multicolumn{2}{|c|}{ BarrierImpact_Mod } & $\begin{array}{l}\text { The respondent indicated they are } \\
\text { moderately affected by barriers to } \\
\text { engaging in cybersecurity behaviours. }\end{array}$ \\
\hline & & \multicolumn{2}{|c|}{ BarrierImpact_High } & $\begin{array}{l}\text { The respondent indicated they are } \\
\text { highly affected by barriers to engaging } \\
\text { in cybersecurity behaviours. }\end{array}$ \\
\hline & & \multicolumn{2}{|c|}{ BarrierImpact_Unsure } & $\begin{array}{l}\text { The respondent indicated they are } \\
\text { unsure about how much their security } \\
\text { behaviours are affected by barriers. }\end{array}$ \\
\hline & \multicolumn{3}{|c|}{ Barrier_Overcoming } & $\begin{array}{l}\text { Feelings identified by respondents } \\
\text { regarding overcoming the barriers to } \\
\text { engaging in safer practices that were } \\
\text { identified by the respondents. }\end{array}$ \\
\hline & \multicolumn{3}{|c|}{ Barrier_Perceived } & $\begin{array}{l}\text { Barriers that respondents see as } \\
\text { preventing them from engaging in what } \\
\text { participants consider to be good internet } \\
\text { security behaviours. }\end{array}$ \\
\hline & & \multicolumn{2}{|c|}{ PercBar_FastChanging } & $\begin{array}{l}\text { The speed with which threats (in } \\
\text { particular) change. Also includes the } \\
\text { need to change behaviours in order to } \\
\text { adapt to the changing threat landscape. }\end{array}$ \\
\hline & & \multicolumn{2}{|c|}{ PercBar_Complacent } & $\begin{array}{l}\text { A sense of complacency about internet } \\
\text { security (e.g. what I have must be good } \\
\text { enough). }\end{array}$ \\
\hline & & \multicolumn{2}{|c|}{ PercBar_FinCost } & $\begin{array}{l}\text { Perception that engaging in safer } \\
\text { internet security behaviours is financially } \\
\text { expensive. }\end{array}$ \\
\hline & & \multicolumn{2}{|c|}{ PercBar_DinishReturns } & $\begin{array}{l}\text { Perception that there is little value } \\
\text { in putting time / money / energy } \\
\text { into learning about threats because } \\
\text { there is not enough of a payoff for the } \\
\text { investment in gaining the knowledge. }\end{array}$ \\
\hline
\end{tabular}




\begin{tabular}{|c|c|c|c|}
\hline $\begin{array}{c}\text { Level } \\
1\end{array}$ & $\begin{array}{c}\text { Level } \\
2\end{array}$ & $\begin{array}{c}\text { Level } \\
3\end{array}$ & Definition \\
\hline & & PercBar_NotSusceptible & $\begin{array}{l}\text { Perception that because respondents do } \\
\text { not feel susceptible to internet threats } \\
\text { they are unlikely to take additional steps } \\
\text { to protect themselves. }\end{array}$ \\
\hline & & PercBar_Energy & $\begin{array}{l}\text { Perceived amount of energy required } \\
\text { by the respondent to be up to date on } \\
\text { the various threats and safeguards, the } \\
\text { energy expended to take the safeguards, } \\
\text { and the energy expended to gain the } \\
\text { requisite knowledge. }\end{array}$ \\
\hline & & PercBar_FeelStupid & $\begin{array}{l}\text { "Respondents feel increasingly } \\
\text { "'"stupid"'" as they try to gain more } \\
\text { knowledge about threats since there are } \\
\text { barriers to understanding." }\end{array}$ \\
\hline & & PercBar_Forgetfulness & $\begin{array}{l}\text { Respondents indicating that they simply } \\
\text { forget to take the steps required to } \\
\text { protect themselves. }\end{array}$ \\
\hline & & PercBar_Habit & $\begin{array}{l}\text { Negative or neutral security habits } \\
\text { in this case. For example, the habit } \\
\text { of ignoring updates (neutral) or } \\
\text { downloading from places where there is } \\
\text { a higher than average risk of malware } \\
\text { (negative). }\end{array}$ \\
\hline & & PercBar_KeepUp & $\begin{array}{l}\text { Perception that it is hard to keep up } \\
\text { with the changing threat landscape and } \\
\text { safeguards. }\end{array}$ \\
\hline & & PercBar_NoCloseExp & $\begin{array}{l}\text { Neither the respondent nor someone } \\
\text { close to them has previously experienced } \\
\text { an internet security breach to the best of } \\
\text { the respondent's knowledge. }\end{array}$ \\
\hline & & PercBar_Ignorance & $\begin{array}{l}\text { Perception by the respondent that they } \\
\text { are ignorant of the threats or safeguards } \\
\text { that they need to be aware of in order } \\
\text { to protect their computer from internet } \\
\text { security threats. }\end{array}$ \\
\hline & & PercBar_IntentNoAct & $\begin{array}{l}\text { Safeguards exist that respondents intend } \\
\text { to use but they have not been followed } \\
\text { up on. }\end{array}$ \\
\hline & & PercBar_Jargon & $\begin{array}{l}\text { Difficulty associated with understanding } \\
\text { technical terms related to internet } \\
\text { security. }\end{array}$ \\
\hline & & PercBar_Laziness & $\begin{array}{l}\text { Respondents feel unwilling to work } \\
\text { towards protecting their computers due } \\
\text { to laziness about security. }\end{array}$ \\
\hline
\end{tabular}




\begin{tabular}{|c|c|c|c|c|}
\hline $\begin{array}{c}\text { Level } \\
1\end{array}$ & $\begin{array}{c}\text { Level } \\
2\end{array}$ & $\begin{array}{c}\text { Level } \\
3\end{array}$ & Level 4 & Definition \\
\hline & & \multicolumn{2}{|c|}{ PercBar_NoPrompts } & $\begin{array}{l}\text { Respondents feel less likely to do } \\
\text { anything to protect themselves if they } \\
\text { are not provided with some sort of } \\
\text { prompt (e.g. security message, threat } \\
\text { notification, etc). }\end{array}$ \\
\hline & & \multicolumn{2}{|c|}{ PercBar_NoPersonalExp } & $\begin{array}{l}\text { Respondents who had not previously } \\
\text { experienced an internet security breach } \\
\text { considered this lack of experience as a } \\
\text { barrier to action. }\end{array}$ \\
\hline & & \multicolumn{2}{|c|}{ PercBar_Reliance } & $\begin{array}{l}\text { Respondents depended on others to } \\
\text { take care of the security for them which } \\
\text { lessened the likelihood of them taking } \\
\text { steps to protect themselves. }\end{array}$ \\
\hline & & \multicolumn{2}{|c|}{ PercBar_SWEffects } & $\begin{array}{l}\text { Protective software utilising system } \\
\text { resources and slowing the performance } \\
\text { of the computer (especially when } \\
\text { running scans) prevented the use of it. }\end{array}$ \\
\hline & & \multicolumn{2}{|c|}{ PercBar_SecurityOverload } & $\begin{array}{l}\text { Feeling so overwhelmed with the } \\
\text { available information, the information } \\
\text { pushed at respondents, the requirements } \\
\text { of websites, etc., that at a certain point } \\
\text { respondents begin to ignore security and } \\
\text { stop taking steps to protect themselves. }\end{array}$ \\
\hline & & \multicolumn{2}{|c|}{ PercBar_TechSkill } & $\begin{array}{l}\text { Perception that a high level of technical } \\
\text { skill is required to effectively protect a } \\
\text { computer online. }\end{array}$ \\
\hline & & \multicolumn{2}{|c|}{ PercBar_Time } & $\begin{array}{l}\text { Perception that a high time commitment } \\
\text { required to safeguard a respondent's } \\
\text { computer. }\end{array}$ \\
\hline & & & TimeBar_DoSecurity & $\begin{array}{l}\text { Time requirement associated with } \\
\text { actually taking the steps to protect } \\
\text { the respondent from internet security } \\
\text { threats. }\end{array}$ \\
\hline & & & TimeBar_IncAware & $\begin{array}{l}\text { Time requirement associated with } \\
\text { increasing the respondent's awareness } \\
\text { of the current internet security threats, } \\
\text { how they work, and how they may be } \\
\text { effected by them. }\end{array}$ \\
\hline & & & TimeBar_LearnSafe & $\begin{array}{l}\text { Time requirement associated with } \\
\text { learning how to effectively use the } \\
\text { safeguards (including both technological } \\
\text { and social safeguards). }\end{array}$ \\
\hline \multicolumn{5}{|c|}{ Beliefs } \\
\hline & \multicolumn{3}{|c|}{ Belief_ExpectAssumptions } & $\begin{array}{l}\text { Expectations and assumptions made by } \\
\text { respondents. }\end{array}$ \\
\hline
\end{tabular}




\begin{tabular}{|c|c|c|c|c|}
\hline $\begin{array}{c}\text { Level } \\
1\end{array}$ & $\begin{array}{c}\text { Level } \\
2\end{array}$ & $\begin{array}{c}\text { Level } \\
3\end{array}$ & Level 4 & Definition \\
\hline & & \multicolumn{2}{|c|}{ Assumpt_BanksFix } & $\begin{array}{l}\text { Banks will fix any financial problems } \\
\text { that occur because of cybersecurity } \\
\text { breaches. }\end{array}$ \\
\hline & & \multicolumn{2}{|c|}{ Assumpt_BanksNotify } & $\begin{array}{l}\text { Gredit card companies will notify you of } \\
\text { anomalous activity on your credit card } \\
\text { quickly. }\end{array}$ \\
\hline & & \multicolumn{2}{|c|}{ Assumpt_PrivacyExpected } & $\begin{array}{l}\text { Expectation of privacy that individuals } \\
\text { have regarding their data. }\end{array}$ \\
\hline & & \multicolumn{2}{|c|}{ Assumpt_GoodSecurityExpensive } & Only costly security is worthwhile. \\
\hline & & \multicolumn{2}{|c|}{ Assumpt_HopeNothingNegative } & $\begin{array}{l}\text { The hope that nothing negative (e.g. a } \\
\text { security breach) happens. }\end{array}$ \\
\hline & & \multicolumn{2}{|c|}{ Assumpt_HopeSWWorks } & $\begin{array}{l}\text { The hope that the protective software } \\
\text { utilised will successfully protect against } \\
\text { all threats. }\end{array}$ \\
\hline & & \multicolumn{2}{|c|}{ Assumpt_MacsNotVulnerable } & $\begin{array}{l}\text { Mac computers are not vulnerable to } \\
\text { viruses and other malware. }\end{array}$ \\
\hline & & \multicolumn{2}{|c|}{ Assumpt_FreeSWSecurity } & $\begin{array}{l}\text { Open source software and freeware is } \\
\text { less secure. }\end{array}$ \\
\hline & & \multicolumn{2}{|c|}{ Assumpt_Password } & $\begin{array}{l}\text { Having a password will protect you from } \\
\text { security threats. }\end{array}$ \\
\hline & & \multicolumn{2}{|c|}{ Assumpt_PeopleTrustworthy } & $\begin{array}{l}\text { Overall belief that people online are } \\
\text { generally trustworthy. }\end{array}$ \\
\hline & & \multicolumn{2}{|c|}{ Assumpt_CompleteProtection } & $\begin{array}{l}\text { If you have protective software installed } \\
\text { on your computer you do not have to } \\
\text { worry about a security breach occurring. }\end{array}$ \\
\hline & & \multicolumn{2}{|c|}{ Assumpt_InherentTrustworthy } & $\begin{array}{l}\text { Some websites are inherently } \\
\text { trustworthy. }\end{array}$ \\
\hline & & \multicolumn{2}{|c|}{ Assumpt_SomeoneElse } & $\begin{array}{l}\text { Someone other than the respondent is } \\
\text { responsible for worrying about keeping } \\
\text { the computer safe. }\end{array}$ \\
\hline \multicolumn{4}{|c|}{ Belief_Negative } & Negative security feelings and beliefs. \\
\hline & & \multicolumn{2}{|c|}{ BelNeg_NoConcernExp } & $\begin{array}{l}\text { Respondent is not concerned about } \\
\text { security until it happens to them or } \\
\text { someone close to them. }\end{array}$ \\
\hline & & \multicolumn{2}{|c|}{ BelNeg_DoLittle } & $\begin{array}{l}\text { Feeling that not much needs to be done } \\
\text { to protect respondents' internet security. }\end{array}$ \\
\hline & & \multicolumn{2}{|c|}{ BelNeg_DoNotDo } & $\begin{array}{l}\text { Respondents do not do what they know } \\
\text { that they should do to protect themselves } \\
\text { online. }\end{array}$ \\
\hline & & \multicolumn{2}{|c|}{ BenNeg_NoDepth } & $\begin{array}{l}\text { Respondent does not look into the } \\
\text { threats / risks online in depth. } \\
\end{array}$ \\
\hline & & \multicolumn{2}{|c|}{ BelNeg_NoAttention } & $\begin{array}{l}\text { Respondents do not pay attention to } \\
\text { threats. }\end{array}$ \\
\hline
\end{tabular}




\begin{tabular}{|c|c|c|c|c|}
\hline $\begin{array}{c}\text { Level } \\
1\end{array}$ & $\begin{array}{c}\text { Level } \\
2\end{array}$ & $\begin{array}{c}\text { Level } \\
3\end{array}$ & Level 4 & Definition \\
\hline & & \multicolumn{2}{|c|}{ BelNeg_ThreatsNotSerious } & $\begin{array}{l}\text { Respondent does not take threats online } \\
\text { seriously. }\end{array}$ \\
\hline & & \multicolumn{2}{|c|}{ BelNeg_FutureConcernPossible } & $\begin{array}{l}\text { Respondent is not currently concerned } \\
\text { about threats, but they may be } \\
\text { concerned about them in the future. }\end{array}$ \\
\hline & & \multicolumn{2}{|c|}{ BelNeg_NotConcerned } & $\begin{array}{l}\text { Overall attitude that the respondent is } \\
\text { not concerned about security online. }\end{array}$ \\
\hline & & \multicolumn{2}{|c|}{ BelNeg_SecurityDifficult } & Security is difficult to accomplish. \\
\hline & & \multicolumn{2}{|c|}{ BelNeg_SecurityForGranted } & Respondent takes security for granted. \\
\hline & & \multicolumn{2}{|c|}{ BelNeg_TechChangesTooQuick } & $\begin{array}{l}\text { Technology changes too quickly for } \\
\text { steps taken by the respondent to be } \\
\text { effective in protecting cybersecurity. }\end{array}$ \\
\hline & & \multicolumn{2}{|c|}{ BelNeg_EasyWayOut } & $\begin{array}{l}\text { Respondent prefers to take the easiest } \\
\text { way to use their computer. }\end{array}$ \\
\hline & & \multicolumn{2}{|c|}{ BelNeg_UnlikelyTarget } & $\begin{array}{l}\text { Respondent belief that they are unlikely } \\
\text { to be attacked online. }\end{array}$ \\
\hline & & \multicolumn{2}{|c|}{ BelNeg_HurtComputerNotPerson } & $\begin{array}{l}\text { Things that cause problems with the } \\
\text { computer it do not hurt the individual. }\end{array}$ \\
\hline & \multicolumn{3}{|c|}{ Belief_PartCharacteristics } & $\begin{array}{l}\text { Self-assessed characteristics of } \\
\text { participants in relation to security. }\end{array}$ \\
\hline & & \multicolumn{2}{|c|}{ BelSelf_Cynical } & $\begin{array}{l}\text { Cynical approach towards security and } \\
\text { actions online. }\end{array}$ \\
\hline & & \multicolumn{2}{|c|}{ BelSelf_Distrustful } & $\begin{array}{l}\text { Distrustful of individuals and websites } \\
\text { online. }\end{array}$ \\
\hline & & \multicolumn{2}{|c|}{ BelSelf_Fearful } & Fearful of threats and being online. \\
\hline & & \multicolumn{2}{|c|}{ BelSelf_Naive } & $\begin{array}{l}\text { Naïve about computers and internet } \\
\text { security. }\end{array}$ \\
\hline & & \multicolumn{2}{|c|}{ BelSelf_NotWebSavvy } & $\begin{array}{l}\text { Not web-savvy and do not understand } \\
\text { the intricacies of online security. }\end{array}$ \\
\hline & & \multicolumn{2}{|c|}{ BelSelf_Paranoid } & Feelings of paranoia online. \\
\hline & & \multicolumn{2}{|c|}{ BelSelf_Proactive } & $\begin{array}{l}\text { Proactive about security (e.g. takes } \\
\text { protective steps to prevent something } \\
\text { from happening). }\end{array}$ \\
\hline & & \multicolumn{2}{|c|}{ BelSelf_Reactive } & $\begin{array}{l}\text { Reactive to security events (e.g. acts after } \\
\text { something has happened). } \\
\end{array}$ \\
\hline & & \multicolumn{2}{|c|}{ BelSelf_RiskAdverse } & Generally avoids risk online. \\
\hline & & \multicolumn{2}{|c|}{ BelSelf_Trusting } & $\begin{array}{l}\text { Generally trusting of people online } \\
\text { (regardless of whether they know the } \\
\text { person they are interacting with). }\end{array}$ \\
\hline & \multicolumn{3}{|c|}{ Belief_Positive } & Positive security beliefs and feelings. \\
\hline
\end{tabular}




\begin{tabular}{|c|c|c|c|}
\hline $\begin{array}{c}\text { Level } \\
1\end{array}$ & \begin{tabular}{|c|} 
Level \\
2 \\
\end{tabular} & \begin{tabular}{c|c} 
Level & Level 4 \\
3 & \\
\end{tabular} & Definition \\
\hline & & Positive_DiscussionIncConcern & $\begin{array}{l}\text { The discussion itself raised the } \\
\text { respondent's awareness enough that they } \\
\text { indicated that it increased their concern } \\
\text { about security. }\end{array}$ \\
\hline & & Positive_Careful & $\begin{array}{l}\text { Respondents felt the need to be } \\
\text { generally careful online in order to avoid } \\
\text { threats. }\end{array}$ \\
\hline & & Positive_SecurityCulture & $\begin{array}{l}\text { A culture that focuses on online security } \\
\text { and examples of security culture. }\end{array}$ \\
\hline & \multicolumn{2}{|c|}{ Belief_Safeguards } & Feelings and beliefs about safeguards. \\
\hline & & BelSG_Hassle & Safeguards are a hassle. \\
\hline & & BelSG_Annoying & Utilising safeguards is annoying. \\
\hline & & BelSG_OneTime & $\begin{array}{l}\text { Safeguards only needs to be taken care } \\
\text { of once. }\end{array}$ \\
\hline & \multicolumn{2}{|c|}{ Beliefs_Threats } & Feelings and beliefs about threats. \\
\hline & & BelThreat_ManyThreats & $\begin{array}{l}\text { There are a lot of threats that users } \\
\text { need to be aware of. }\end{array}$ \\
\hline & & BelThreat_MajorThreats & $\begin{array}{l}\text { Major threats are more worthy of } \\
\text { attention than minor threats. }\end{array}$ \\
\hline & & BelThreat_NotNice & Security breaches are "not nice". \\
\hline & & BelThreat_HardToRid & Threats are difficult to get rid of. \\
\hline & & BelThreat_QuickChange & Threats can change quickly. \\
\hline & & BelThreat_MoreOffline & $\begin{array}{l}\text { Threats are more likely to occur offline } \\
\text { than online. }\end{array}$ \\
\hline & & BelThreat_Unaware & $\begin{array}{l}\text { The respondent is unaware of threats } \\
\text { that they may be faced with online. }\end{array}$ \\
\hline & & BelThreat_UnsureSteps & $\begin{array}{l}\text { The respondent is unsure of how to deal } \\
\text { with threats if a breach does occur. }\end{array}$ \\
\hline \multicolumn{4}{|c|}{ Benefits } \\
\hline & \multicolumn{2}{|c|}{ Benefit_LessWorry } & $\begin{array}{l}\text { Taking safeguards means that there } \\
\text { is one less thing for the respondent to } \\
\text { worry about online. }\end{array}$ \\
\hline & \multicolumn{2}{|c|}{ Benefit_Reassurance } & $\begin{array}{l}\text { Taking safeguards offers participants a } \\
\text { level of reassurance that they will not } \\
\text { have to deal with a threat / breach. }\end{array}$ \\
\hline & \multicolumn{2}{|c|}{ Benefit_Unsure } & $\begin{array}{l}\text { Benefits of taking safeguards are } \\
\text { unclear. }\end{array}$ \\
\hline \multicolumn{4}{|c|}{ Confidence } \\
\hline & \multicolumn{2}{|c|}{ Confidence_Low } & $\begin{array}{l}\text { Indicative of a low level of confidence } \\
\text { about the skills held by a respondent. }\end{array}$ \\
\hline & \multicolumn{2}{|c|}{ Confidence_Mod } & $\begin{array}{l}\text { Indicative of a moderate level of } \\
\text { confidence about the skills held by a } \\
\text { respondent. }\end{array}$ \\
\hline
\end{tabular}




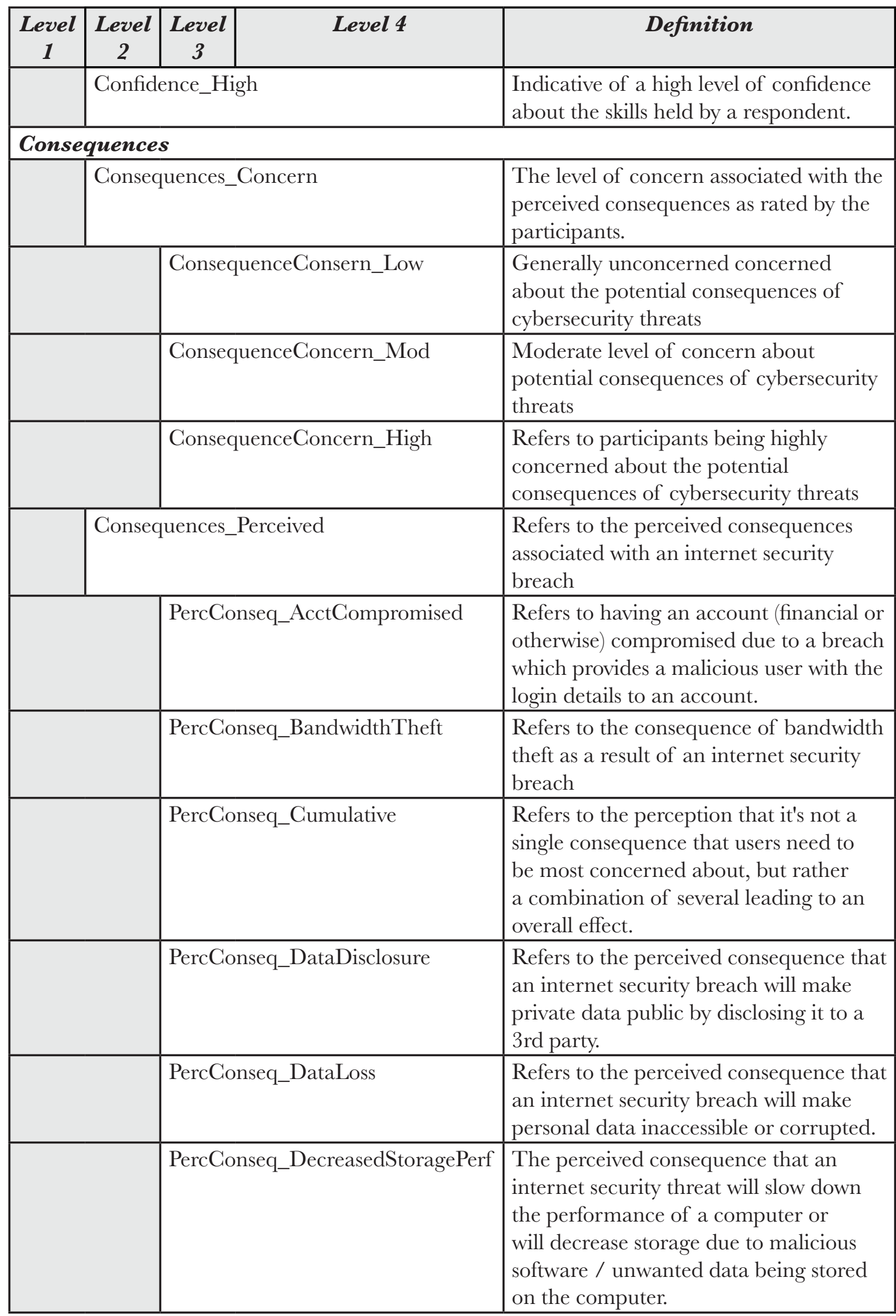




\begin{tabular}{|c|c|c|c|c|}
\hline $\begin{array}{c}\text { Level } \\
1\end{array}$ & \begin{tabular}{|c|} 
Level \\
2 \\
\end{tabular} & $\begin{array}{c}\text { Level } \\
3\end{array}$ & Level 4 & Definition \\
\hline & & \multicolumn{2}{|c|}{ PercConseq_Financial } & $\begin{array}{l}\text { Perceived financial consequences } \\
\text { associated with an internet security } \\
\text { breach }\end{array}$ \\
\hline & & & FinConseq_AcctAccess & $\begin{array}{l}\text { The potential financial consequences } \\
\text { associated with having your banking } \\
\text { details stolen due to an internet security } \\
\text { threat. }\end{array}$ \\
\hline & & & FinConseq_HWPurch & $\begin{array}{l}\text { The perception that in order to recover } \\
\text { from a security breach it may require } \\
\text { purchasing new hardware (e.g. a new } \\
\text { computer) }\end{array}$ \\
\hline & & & FinConseq_SoftwarePurch & $\begin{array}{l}\text { Costs associated with buying better or } \\
\text { more up to date security software in } \\
\text { order to recover from a breach. }\end{array}$ \\
\hline & & & FinConseq_StolenCC & $\begin{array}{l}\text { Consequences associated with having } \\
\text { your credit card information stolen due } \\
\text { to an internet security threat. }\end{array}$ \\
\hline & & & FinConseq_OtherPurch & $\begin{array}{l}\text { Spending additional money on } \\
\text { something for yourself that you might } \\
\text { not have otherwise had to pay for. This } \\
\text { does not refer to purchases made by } 3 r d \\
\text { parties rather than those made by you. }\end{array}$ \\
\hline & & \multicolumn{2}{|c|}{ PercConseq_IDTheft } & $\begin{array}{l}\text { Identity theft caused by an internet } \\
\text { security breach. }\end{array}$ \\
\hline & & \multicolumn{2}{|c|}{ PercConseq_InfectedComp } & $\begin{array}{l}\text { Perception that the consequence of an } \\
\text { internet security threat is an infected } \\
\text { computer. }\end{array}$ \\
\hline & & \multicolumn{2}{|c|}{ PercConseq_RepHarm } & $\begin{array}{l}\text { Potential loss of reputation that a person } \\
\text { has earned, due to an internet security } \\
\text { breach. }\end{array}$ \\
\hline & & \multicolumn{2}{|c|}{ PercConseq_TimeCost } & $\begin{array}{l}\text { Time required to return to the status } \\
\text { quo as a consequence of an internet } \\
\text { security breach. }\end{array}$ \\
\hline & & & TimeConseq_Prevent & $\begin{array}{l}\text { Time required to install software } \\
\text { protections and maintain safe } \\
\text { behaviours in order to prevent (as much } \\
\text { as possible) the occurrence of a future } \\
\text { cybersecurity breach. }\end{array}$ \\
\hline & & & TimeConseq_Repair & $\begin{array}{l}\text { Time required to fix problems caused by } \\
\text { an internet security breach (specifically, } \\
\text { technical fixes). }\end{array}$ \\
\hline & & \multicolumn{2}{|c|}{ PercConseq_TransferMalware } & $\begin{array}{l}\text { Possibility of an internet security breach } \\
\text { at home contributing to an internet } \\
\text { security breach at work. }\end{array}$ \\
\hline
\end{tabular}




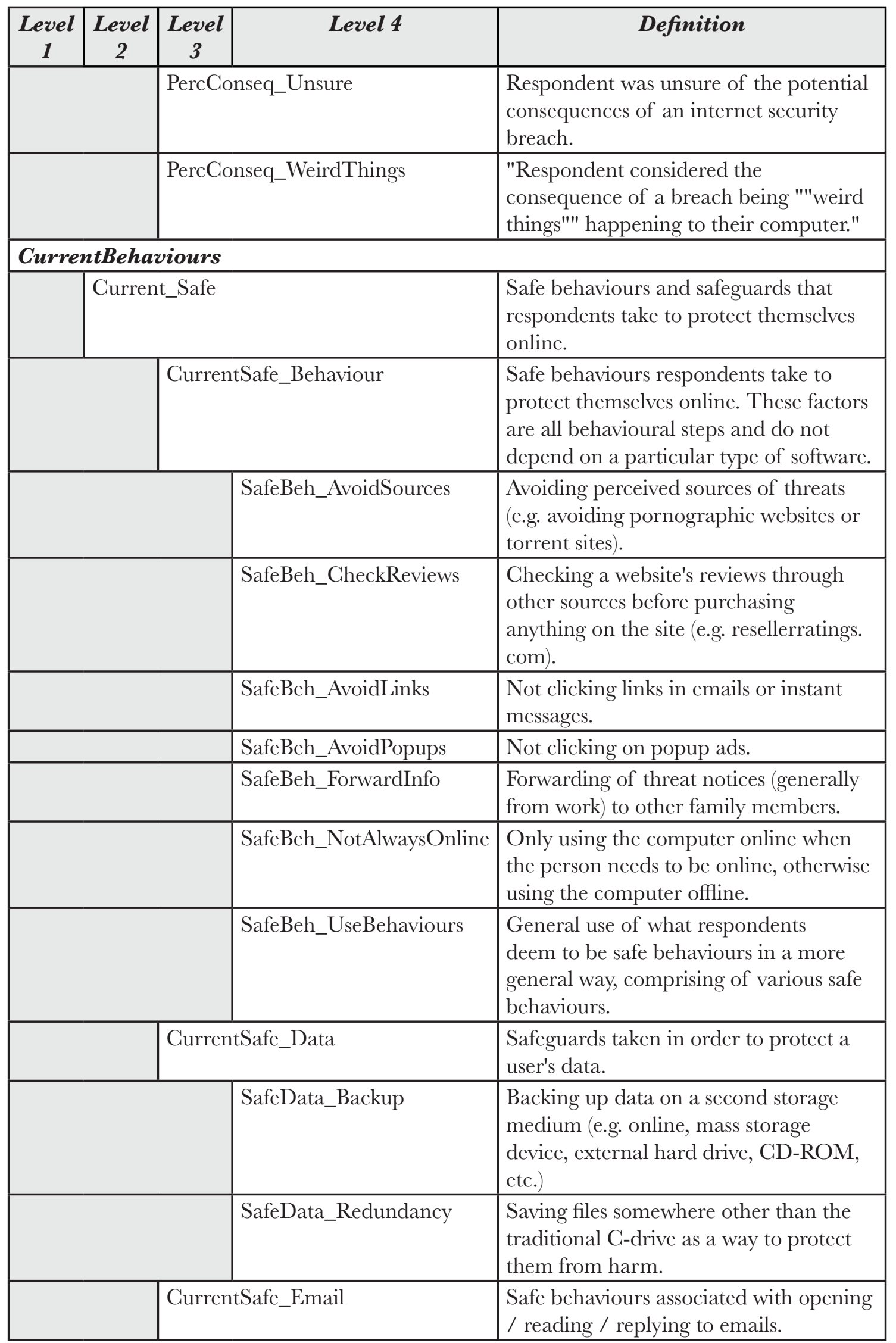




\begin{tabular}{|c|c|c|c|c|}
\hline $\begin{array}{c}\text { Level } \\
1\end{array}$ & $\begin{array}{c}\text { Level } \\
2\end{array}$ & $\begin{array}{c}\text { Level } \\
3\end{array}$ & Level 4 & Definition \\
\hline & & & SafeEmail_Suspicious & Not opening suspicious emails. \\
\hline & & & SafeEmail_Attachments & $\begin{array}{l}\text { Not opening email attachments that the } \\
\text { person was not expecting. }\end{array}$ \\
\hline & & & SafeEmail_Unknown & $\begin{array}{l}\text { Not opening emails if the person does } \\
\text { not know who has sent it. }\end{array}$ \\
\hline & & \multicolumn{2}{|c|}{ CurrentSafe_Passwords } & $\begin{array}{l}\text { Current safe password behaviours } \\
\text { indicated by respondents. }\end{array}$ \\
\hline & & & SafePass_Change & Changing a password regularly. \\
\hline & & & SafePass_AvoidWriting & Not writing down your password. \\
\hline & & & SafePass_Different & $\begin{array}{l}\text { Using different passwords fro different } \\
\text { sites. }\end{array}$ \\
\hline & & & SafePass_Matrices & $\begin{array}{l}\text { Use of a coding scheme to create } \\
\text { difficult passwords. }\end{array}$ \\
\hline & & \multicolumn{2}{|c|}{ CurrentSafe_Privacy } & $\begin{array}{l}\text { Practices related to securing an } \\
\text { individual's privacy. }\end{array}$ \\
\hline & & & DataPrivacy_Disclosure & $\begin{array}{l}\text { Consideration by the respondent of the } \\
\text { potential for disclosure of information. }\end{array}$ \\
\hline & & & DataPrivacy_NoSensitive & $\begin{array}{l}\text { Not putting sensitive information on } \\
\text { the computer in case the computer is } \\
\text { compromised. }\end{array}$ \\
\hline & & & IDPrivacy_NoPublicising & $\begin{array}{l}\text { Not making private information public } \\
\text { (e.g. not posting your home address on } \\
\text { the internet). }\end{array}$ \\
\hline & & & IDPrivacy_NoRealName & $\begin{array}{l}\text { Using a false name when a name is } \\
\text { required online. }\end{array}$ \\
\hline & & \multicolumn{2}{|c|}{ CurrentSafe_Software } & $\begin{array}{l}\text { Practices relating to computer software } \\
\text { and keeping the computer safe. }\end{array}$ \\
\hline & & & SafeSoft_Updated & Antivirus software is kept up to date. \\
\hline & & & SafeSoft_ProtectiveSW & $\begin{array}{l}\text { Use of protective software (e.g. antivirus, } \\
\text { firewall, antiadware, etc.). }\end{array}$ \\
\hline & & \multicolumn{2}{|c|}{ GurrentSafe_Web } & $\begin{array}{l}\text { Safe behaviours associated with using } \\
\text { websites. }\end{array}$ \\
\hline & & & SafeWeb_NoBillPayment & Not paying bills online. \\
\hline & & & SafeWeb_NoBanking & Not using online banking, \\
\hline & & & SafeWeb_TrustedSites & Only visiting trusted websites. \\
\hline & \multicolumn{3}{|c|}{ Current_Unsafe } & $\begin{array}{l}\text { Unsafe behaviours that users currently } \\
\text { engage in with regard to their } \\
\text { behaviours, data practices, password } \\
\text { practices, software practices, and trust. }\end{array}$ \\
\hline & & \multicolumn{2}{|c|}{ CurrentUnsafe_Behaviours } & Unsafe behaviours \\
\hline & & & UnsafeBeh_NoStepsTaken & $\begin{array}{l}\text { Not actively taking any steps to secure a } \\
\text { home computers (non-action). }\end{array}$ \\
\hline
\end{tabular}




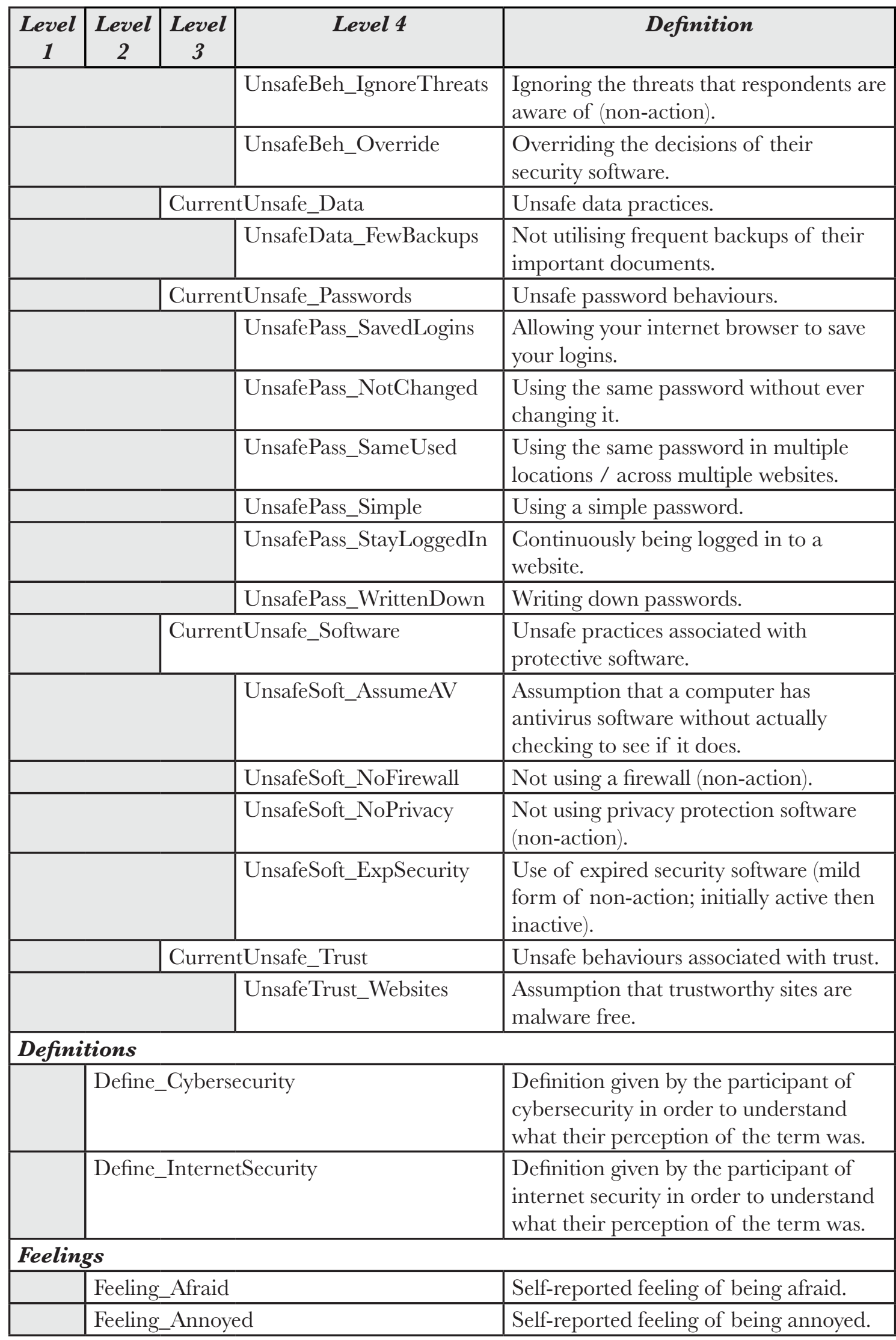




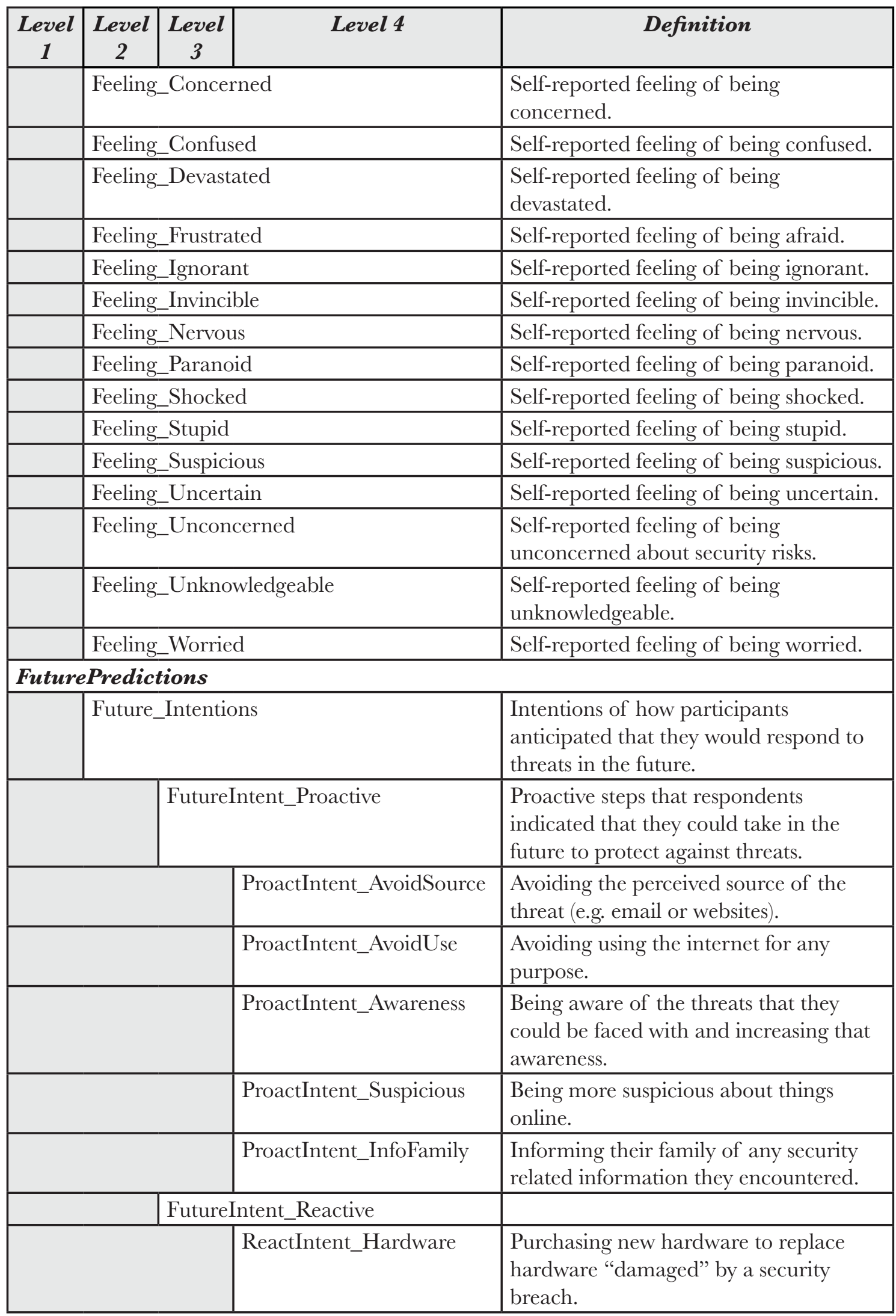




\begin{tabular}{|c|c|c|c|c|}
\hline $\begin{array}{c}\text { Level } \\
1\end{array}$ & \begin{tabular}{|c|} 
Level \\
2
\end{tabular} & $\begin{array}{c}\text { Level } \\
3\end{array}$ & Level 4 & Definition \\
\hline & & & ReactIntent_Software & $\begin{array}{l}\text { Purchasing new software to remedy the } \\
\text { resulting consequences of a security } \\
\text { breach. }\end{array}$ \\
\hline & & & ReactIntent_DecTrust & $\begin{array}{l}\text { Decreasing the trust held in online } \\
\text { sources. }\end{array}$ \\
\hline & & & ReactIntent_KeepUsing & $\begin{array}{l}\text { Continued use of the internet with little } \\
\text { change. }\end{array}$ \\
\hline & & & ReactIntent_NotConnect & $\begin{array}{l}\text { Physically disconnect from the internet, } \\
\text { only connecting as needed (rather than } \\
\text { always on). }\end{array}$ \\
\hline & & & ReactIntent_StopUsing & $\begin{array}{l}\text { Stop using the internet or the service } \\
\text { that is perceived to be risky. }\end{array}$ \\
\hline & & & ReactIntent_NoChange & $\begin{array}{l}\text { Continued use of the internet with no } \\
\text { change to behaviours. }\end{array}$ \\
\hline & Future & Likeliho & & $\begin{array}{l}\text { The perceived likelihood that } \\
\text { respondents would encounter a } \\
\text { cybersecurity threat in the future. }\end{array}$ \\
\hline & & FutureI & ike_Low & $\begin{array}{l}\text { Low perceived likelihood of } \\
\text { encountering a cybersecurity threat. }\end{array}$ \\
\hline & & FutureI & ike_Mod & $\begin{array}{l}\text { Moderate perceived likelihood of } \\
\text { encountering a cybersecurity threat. }\end{array}$ \\
\hline & & FutureI & ike_High & $\begin{array}{l}\text { High perceived likelihood of } \\
\text { encountering a cybersecurity threat. }\end{array}$ \\
\hline & Future & Predicti & ons & $\begin{array}{l}\text { Predictions made by respondents about } \\
\text { the future threat landscape. }\end{array}$ \\
\hline Hardr & vare & & & \\
\hline & Hardw: & are_Mol & & Mobile Phones, Tablets, iPods \\
\hline & Hardw: & are_Mac & & $\begin{array}{l}\text { Apple computers running a version of } \\
\text { Mac OS }\end{array}$ \\
\hline & Hardw: & are_PC & & $\begin{array}{l}\text { PG computers (all PG computers } \\
\text { were running a version of Microsoft } \\
\text { Windows) }\end{array}$ \\
\hline Inforn & nations & Sources & & \\
\hline & InfoSou & urce_Inte & ernet & $\begin{array}{l}\text { Internet based sources of information } \\
\text { about security threats. }\end{array}$ \\
\hline & & Interne & tInfo_Google & Google. \\
\hline & & Interne & tInfo_Govt & Government websites. \\
\hline & & Interne & tInfo_General & $\begin{array}{l}\text { General information available on the } \\
\text { internet (e.g. blogs, security sites, etc.) }\end{array}$ \\
\hline & & Interne & tInfo_Wiki & Wikipedia. \\
\hline & InfoSou & urce_Otl & & $\begin{array}{l}\text { Other sources of information about } \\
\text { security threats. }\end{array}$ \\
\hline & & OtherIr & afo_YellowPages & Yellow Pages. \\
\hline
\end{tabular}




\begin{tabular}{|c|c|c|c|c|}
\hline $\begin{array}{c}\text { Level } \\
1\end{array}$ & $\begin{array}{c}\text { Level } \\
2\end{array}$ & $\begin{array}{c}\text { Level } \\
3\end{array}$ & Level 4 & Definition \\
\hline & \multicolumn{3}{|c|}{ InfoSource_Social } & $\begin{array}{l}\text { Social-based sources of information } \\
\text { about security threats. }\end{array}$ \\
\hline & & \multicolumn{2}{|c|}{ SocialInfo_Colleague } & Colleagues at work. \\
\hline & & \multicolumn{2}{|c|}{ SocialInfo_Family } & Family members. \\
\hline & & & FamilyInfo_Child & $\begin{array}{l}\text { Information sought from children of } \\
\text { respondents. }\end{array}$ \\
\hline & & & FamilyInfo_Relative & $\begin{array}{l}\text { Information sought from other relatives } \\
\text { of respondents. }\end{array}$ \\
\hline & & & FamilyInfo_Parent & $\begin{array}{l}\text { Information sought from parents of } \\
\text { respondents. }\end{array}$ \\
\hline & & & FamilyInfo_Husband & $\begin{array}{l}\text { Information sought from respondents' } \\
\text { husbands. }\end{array}$ \\
\hline & & & FamilyInfo_Partner & $\begin{array}{l}\text { Information sought from respondents' } \\
\text { partners. }\end{array}$ \\
\hline & & & FamilyInfo_Wife & $\begin{array}{l}\text { Information sought from respondents' } \\
\text { wives. }\end{array}$ \\
\hline & & \multicolumn{2}{|c|}{ SocialInfo_Friend } & $\begin{array}{l}\text { Information sought from friends of } \\
\text { respondents. }\end{array}$ \\
\hline & \multicolumn{3}{|c|}{ InfoSource_Experts } & $\begin{array}{l}\text { Information sought from perceived } \\
\text { security experts. }\end{array}$ \\
\hline & & \multicolumn{2}{|c|}{$\begin{array}{l}\text { ExpertInfo_ } \\
\text { HardwareManufacturer }\end{array}$} & $\begin{array}{l}\text { Information from the hardware } \\
\text { manufacturer (e.g. Apple, Dell, IBM). }\end{array}$ \\
\hline & & \multicolumn{2}{|c|}{ ExpertInfo_Retailer } & Information from a technology retailer. \\
\hline & & \multicolumn{2}{|c|}{ ExpertInfo_TechExpert } & $\begin{array}{l}\text { Information from a perceived technical } \\
\text { expert (may also be a family member } \\
\text { or colleague, but the difference is that } \\
\text { they are perceived to be an expert in the } \\
\text { subject area for some reason). }\end{array}$ \\
\hline & \multicolumn{3}{|c|}{ InfoSource_TradMedia } & Traditional sources of information. \\
\hline & & \multicolumn{2}{|c|}{ InfoMedia_Book } & Books. \\
\hline & & \multicolumn{2}{|c|}{ InfoMedia_Magazine } & Magazines. \\
\hline & & \multicolumn{2}{|c|}{ InfoMedia_News } & Newspapers, TV news, Internet news. \\
\hline \multicolumn{5}{|c|}{ Knozvledge } \\
\hline & \multicolumn{3}{|c|}{ Knowledge_Good } & $\begin{array}{l}\text { Correct and well informed knowledge } \\
\text { by respondents about security threats } \\
\text { and safeguards. }\end{array}$ \\
\hline & & \multicolumn{2}{|c|}{ GoodKnow_Proactive } & $\begin{array}{l}\text { Respondents indicated that they knew } \\
\text { that it was good to be proactive rather } \\
\text { than reactive in terms of security. }\end{array}$ \\
\hline & & \multicolumn{2}{|c|}{ GoodKnow_RecogniseThreats } & $\begin{array}{l}\text { The respondent demonstrated the } \\
\text { knowledge associated with being able to } \\
\text { identify online threats. }\end{array}$ \\
\hline
\end{tabular}




\begin{tabular}{|c|c|c|c|c|}
\hline $\begin{array}{c}\text { Level } \\
1\end{array}$ & $\begin{array}{c}\text { Level } \\
2\end{array}$ & $\begin{array}{c}\text { Level } \\
3\end{array}$ & Level 4 & Definition \\
\hline & & \multicolumn{2}{|c|}{ GoodKnow_RecogniseScams } & $\begin{array}{l}\text { The respondent demonstrated the } \\
\text { knowledge associated with being able to } \\
\text { identify scams in email and on websites. }\end{array}$ \\
\hline & & \multicolumn{2}{|c|}{ GoodKnow_RecogniseWebsites } & $\begin{array}{l}\text { The respondent demonstrated the } \\
\text { knowledge required to be able to } \\
\text { recognise suspicious websites and to } \\
\text { differentiate them from other websites. }\end{array}$ \\
\hline & & \multicolumn{2}{|c|}{$\begin{array}{l}\text { GoodKnow_- } \\
\text { SoftwareIncompatibility }\end{array}$} & $\begin{array}{l}\text { The respondent demonstrate } \\
\text { knowledge in relation to the potential } \\
\text { for incompatibilities and problems to } \\
\text { arise if multiple versions of protective } \\
\text { software are installed. }\end{array}$ \\
\hline & & \multicolumn{2}{|c|}{ GoodKnow_PermanenceInfo } & $\begin{array}{l}\text { The respondent demonstrated } \\
\text { knowledge that whatever is put online } \\
\text { has the potential to be available to } \\
\text { anyone, forever. }\end{array}$ \\
\hline & & \multicolumn{2}{|c|}{ GoodKnow_UnderstandThreats } & $\begin{array}{l}\text { The respondent demonstrated } \\
\text { the knowledge associated with } \\
\text { understanding threats. }\end{array}$ \\
\hline & & \multicolumn{2}{|c|}{ GoodKnow_VirusesDisguised } & $\begin{array}{l}\text { The respondent demonstrated the } \\
\text { knowledge of how viruses can be } \\
\text { disguised as non-malicious seeming } \\
\text { items. }\end{array}$ \\
\hline & & \multicolumn{2}{|c|}{ GoodKnow_CausesOfSlowComp } & $\begin{array}{l}\text { The respondent demonstrated the } \\
\text { knowledge of understanding that many } \\
\text { things (aside from just malware) can be } \\
\text { responsible for slowing down computers. }\end{array}$ \\
\hline & \multicolumn{3}{|c|}{ Knowledge_Poor } & $\begin{array}{l}\text { Poor, inaccurate, or misleading } \\
\text { knowledge demonstrated by } \\
\text { respondents. }\end{array}$ \\
\hline & & \multicolumn{2}{|c|}{ PoorKnow_WhatWorry } & $\begin{array}{l}\text { The respondent indicated that they did } \\
\text { not know what to be concerned about } \\
\text { online. }\end{array}$ \\
\hline & & \multicolumn{2}{|c|}{ PoorKnow_ThreatUnderstanding } & $\begin{array}{l}\text { The respondent indicated that they did } \\
\text { not know how threats worked (to the } \\
\text { extent the the average person would be } \\
\text { expected to understand ho threats work). }\end{array}$ \\
\hline & \multicolumn{3}{|c|}{ Knowledge_IncorrAssumptions } & $\begin{array}{l}\text { Incorrect assumptions about security } \\
\text { made by respondents }\end{array}$ \\
\hline & \multicolumn{3}{|c|}{ Knowledge_FGQuestions } & $\begin{array}{l}\text { Questions that were raised in the focus } \\
\text { groups that were answered by other } \\
\text { participants. }\end{array}$ \\
\hline
\end{tabular}




\begin{tabular}{|c|c|c|c|c|}
\hline $\begin{array}{c}\text { Level } \\
1\end{array}$ & $\begin{array}{c}\text { Level } \\
2\end{array}$ & $\begin{array}{c}\text { Level } \\
3\end{array}$ & Level 4 & Definition \\
\hline & \multicolumn{3}{|c|}{ Knowledge_Understanding } & $\begin{array}{l}\text { Knowledge associated with } \\
\text { understanding the topics raised (e.g. } \\
\text { when the discussion was on a particular } \\
\text { topic, did the respondent understand it). }\end{array}$ \\
\hline & & Under & & $\begin{array}{l}\text { The respondent demonstrated a low } \\
\text { level of understanding the topic at hand. }\end{array}$ \\
\hline & & Under & certain & $\begin{array}{l}\text { The respondent demonstrated } \\
\text { uncertainty about the topic at hand. }\end{array}$ \\
\hline & & Under & & $\begin{array}{l}\text { The respondent demonstrated a high } \\
\text { level of understanding about the topic. }\end{array}$ \\
\hline \multicolumn{5}{|c|}{ EgoProtection } \\
\hline & \multicolumn{3}{|c|}{ Ego_Luck } & $\begin{array}{l}\text { Perceptions about the luckiness } \\
\text { or unluckiness associated with } \\
\text { cybersecurity breaches. }\end{array}$ \\
\hline & & Luck_l & & $\begin{array}{l}\text { The perception by the participant that } \\
\text { they have been lucky enough to avoid } \\
\text { internet security breaches. }\end{array}$ \\
\hline & & Luck_l & & $\begin{array}{l}\text { The perception by the participant that } \\
\text { they have been unlucky and therefore } \\
\text { have had to deal with some sort of } \\
\text { internet security breach. }\end{array}$ \\
\hline & \multicolumn{3}{|c|}{ Ego_Boasting } & $\begin{array}{l}\text { Boasting about the respondent's security } \\
\text { prowess. }\end{array}$ \\
\hline & \multicolumn{3}{|c|}{ Ego_Downplaying } & $\begin{array}{l}\text { Downplaying the respondent's security } \\
\text { prowess. }\end{array}$ \\
\hline \multicolumn{5}{|c|}{ MaliciousUsers } \\
\hline & \multicolumn{3}{|c|}{ MalUsers_Characteristics } & $\begin{array}{l}\text { Perceived characteristics of malicious } \\
\text { users as described by respondents. }\end{array}$ \\
\hline & & \multicolumn{2}{|c|}{ MalUserChar_Devious } & Malicious users are devious. \\
\hline & & \multicolumn{2}{|c|}{ MalUserChar_FinanciallySecure } & $\begin{array}{l}\text { Malicious users are financially secure / } \\
\text { wealthy. }\end{array}$ \\
\hline & & \multicolumn{2}{|c|}{ MalUserChar_Foreign } & Malicious users are foreigners. \\
\hline & & \multicolumn{2}{|c|}{ MalUserChar_LatestTech } & $\begin{array}{l}\text { Malicious users have the latest } \\
\text { technology available to them. }\end{array}$ \\
\hline & & \multicolumn{2}{|c|}{ MalUserChar_KnowsVictims } & $\begin{array}{l}\text { Malicious users have taken steps to } \\
\text { ensure that they know about their } \\
\text { victims before targeting them. }\end{array}$ \\
\hline & & \multicolumn{2}{|c|}{ MalUserChar_PlansAhead } & $\begin{array}{l}\text { Malicious users take steps to plan ahead } \\
\text { who they will attack and what they hope } \\
\text { to get. }\end{array}$ \\
\hline & & \multicolumn{2}{|c|}{ MalUserChar_Smart } & Malicious users are smart. \\
\hline & & \multicolumn{2}{|c|}{ MalUserChar_Talented } & $\begin{array}{l}\text { Malicious users are talented (regarding } \\
\text { their technology skills). }\end{array}$ \\
\hline
\end{tabular}




\begin{tabular}{|c|c|c|c|c|}
\hline $\begin{array}{c}\text { Level } \\
1\end{array}$ & $\begin{array}{c}\text { Level } \\
2\end{array}$ & $\begin{array}{c}\text { Level } \\
3\end{array}$ & Level 4 & Definition \\
\hline & & \multicolumn{2}{|c|}{ MalUserChar_WellEducated } & $\begin{array}{l}\text { Malicious users have been well educated } \\
\text { which has furthered their skills. }\end{array}$ \\
\hline & & \multicolumn{2}{|c|}{ MalUserChar_Young } & Malicious users are young \\
\hline & \multicolumn{3}{|c|}{ MalUsers_Goals } & $\begin{array}{l}\text { Perceived goals associated with } \\
\text { malicious users behaviours as described } \\
\text { by respondents. }\end{array}$ \\
\hline & & \multicolumn{2}{|c|}{ MalUserGoal_DefaudVictim } & $\begin{array}{l}\text { Malicious users want to defraud their } \\
\text { victims (not necessarily financially). }\end{array}$ \\
\hline & & \multicolumn{2}{|c|}{ MalUserGoal_FinancialGain } & $\begin{array}{l}\text { Malicious users want to get money from } \\
\text { their victims. }\end{array}$ \\
\hline & & \multicolumn{2}{|c|}{ MalUserGoal_ProveSkill } & $\begin{array}{l}\text { Malicious users want to prove their skills } \\
\text { to other malicious users. }\end{array}$ \\
\hline & & \multicolumn{2}{|c|}{ MalUserGoal_StealInfo } & $\begin{array}{l}\text { Malicious users want to steal their } \\
\text { victims' information. }\end{array}$ \\
\hline & \multicolumn{3}{|c|}{ MalUsers_Motivations } & $\begin{array}{l}\text { The perceived motives behind malicious } \\
\text { users' behaviours as described by } \\
\text { respondents. }\end{array}$ \\
\hline & & \multicolumn{2}{|c|}{ MalUserMotive_NothingToLose } & $\begin{array}{l}\text { Malicious users have nothing to lose by } \\
\text { engaging in malicious behaviours. }\end{array}$ \\
\hline & & \multicolumn{2}{|c|}{ MalUserMotive_Prank } & $\begin{array}{l}\text { Malicious users simply want to prank } \\
\text { (a very non-malicious outcome) their } \\
\text { victims. }\end{array}$ \\
\hline & & \multicolumn{2}{|c|}{ MalUserMotive_ShowClever } & $\begin{array}{l}\text { Malicious users want to show their } \\
\text { victims and other malicious users that } \\
\text { they are clever. }\end{array}$ \\
\hline & \multicolumn{3}{|c|}{ MalUsers_Opinions } & $\begin{array}{l}\text { Opinions about malicious users } \\
\text { described by respondents. }\end{array}$ \\
\hline & & \multicolumn{2}{|c|}{ MalUserOpin_LeerIn } & $\begin{array}{l}\text { Malicious users leer into their victims } \\
\text { lives. }\end{array}$ \\
\hline & & \multicolumn{2}{|c|}{ MalUserOpin_IndivLowValue } & $\begin{array}{l}\text { Malicious users will not target } \\
\text { individuals that are of low value (e.g. no } \\
\text { valuable data, not rich, etc.) }\end{array}$ \\
\hline & & \multicolumn{2}{|c|}{ MalUserOpin_NotNice } & Malicious users are not nice individuals. \\
\hline & \multicolumn{3}{|c|}{ MalUsers_Tactics } & $\begin{array}{l}\text { Tactics respondents believed that } \\
\text { malicious users could use to attack them. }\end{array}$ \\
\hline & & \multicolumn{2}{|c|}{ MalUserTactuc_UseSecuritySite } & $\begin{array}{l}\text { The use of fake security sites to } \\
\text { install malware or provide incorrect } \\
\text { information to respondents. }\end{array}$ \\
\hline & \multicolumn{3}{|c|}{ MalUsers_Targets } & The perceived targets of malicious users. \\
\hline & & \multicolumn{2}{|c|}{ MalUserTarget_Institutions } & Institutions and businesses. \\
\hline & & \multicolumn{2}{|c|}{ MalUserTarget_PCUsers } & $\begin{array}{l}\text { People who use PCs (e.g. not Mac or } \\
\text { Linux users). }\end{array}$ \\
\hline & & \multicolumn{2}{|c|}{ MalUserTarget_Rich } & People who are rich. \\
\hline
\end{tabular}




\begin{tabular}{|c|c|c|c|}
\hline $\begin{array}{c}\text { Level } \\
1\end{array}$ & \begin{tabular}{|c|} 
Level \\
2 \\
\end{tabular} & $\begin{array}{c}\text { Level } \\
3\end{array}$ & Definition \\
\hline \multicolumn{4}{|c|}{ Notifications } \\
\hline & \multicolumn{2}{|c|}{ Notif_Error } & $\begin{array}{l}\text { Participants indicated that they had seen } \\
\text { error messages in relation to their online } \\
\text { security. }\end{array}$ \\
\hline & \multicolumn{2}{|c|}{ Notif_None } & $\begin{array}{l}\text { Participants indicated that they had not } \\
\text { seen any notifications that would suggest } \\
\text { that their online security was at risk. }\end{array}$ \\
\hline & \multicolumn{2}{|c|}{ Notif_Software } & $\begin{array}{l}\text { Participants indicated that they had } \\
\text { seen messages originating from software } \\
\text { (typically protective software) about their } \\
\text { security. }\end{array}$ \\
\hline & \multicolumn{2}{|c|}{ Notif_System } & $\begin{array}{l}\text { Participants indicated that they had seen } \\
\text { operating system messages in relation to } \\
\text { their online security (e.g. a notification to } \\
\text { turn on Windows firewall). }\end{array}$ \\
\hline \multicolumn{4}{|c|}{ PerceivedSeverity } \\
\hline & \multicolumn{2}{|c|}{ Severity_Low } & $\begin{array}{l}\text { The perceived severity of the } \\
\text { consequences of a cybersecurity breach } \\
\text { was low. }\end{array}$ \\
\hline & \multicolumn{2}{|c|}{ Severity_Mod } & $\begin{array}{l}\text { The perceived severity of the } \\
\text { consequences of a cybersecurity breach } \\
\text { was moderate. }\end{array}$ \\
\hline & \multicolumn{2}{|c|}{ Severity_High } & $\begin{array}{l}\text { The perceived severity of the } \\
\text { consequences of a cybersecurity breach } \\
\text { was high. }\end{array}$ \\
\hline \multicolumn{4}{|c|}{ PerceivedSusceptibility } \\
\hline & \multicolumn{2}{|c|}{ Susceptibility_Low } & $\begin{array}{l}\text { The respondent considered the } \\
\text { likelihood of experiencing a } \\
\text { cybersecurity breach to be low. }\end{array}$ \\
\hline & \multicolumn{2}{|c|}{ Susceptibility_Mod } & $\begin{array}{l}\text { The respondent considered the } \\
\text { likelihood of experiencing a } \\
\text { cybersecurity breach to be moderate. }\end{array}$ \\
\hline & \multicolumn{2}{|c|}{ Susceptibility_High } & $\begin{array}{l}\text { The respondent considered the } \\
\text { likelihood of experiencing a } \\
\text { cybersecurity breach to be high. }\end{array}$ \\
\hline \multicolumn{4}{|c|}{ PriorExperience } \\
\hline & \multicolumn{2}{|c|}{ PriorExp_IncreasedConcern } & $\begin{array}{l}\text { Result of prior experience as increased } \\
\text { concern about threats. }\end{array}$ \\
\hline & \multicolumn{2}{|c|}{ PriorExp_Example } & $\begin{array}{l}\text { Examples of prior experiences that } \\
\text { respondents had with cybersecurity } \\
\text { breaches. }\end{array}$ \\
\hline & \multicolumn{2}{|c|}{ PriorExp_Unsure } & $\begin{array}{l}\text { The respondent indicated that they were } \\
\text { unsure whether they had experienced a } \\
\text { cybersecurity breach in the past. }\end{array}$ \\
\hline
\end{tabular}




\begin{tabular}{|c|c|c|c|}
\hline $\begin{array}{c}\text { Level } \\
1\end{array}$ & $\begin{array}{c}\text { Level } \\
2\end{array}$ & $\begin{array}{c}\text { Level } \\
3\end{array}$ & Definition \\
\hline & \multicolumn{2}{|c|}{ PriorExp_No } & $\begin{array}{l}\text { The respondent indicated that they } \\
\text { believed that they had not experienced } \\
\text { a cybersecurity breach in the past (note: } \\
\text { belief is sufficient). }\end{array}$ \\
\hline & \multicolumn{2}{|c|}{ PriorExp_Yes } & $\begin{array}{l}\text { The respondent indicated that they } \\
\text { believed they had experienced a } \\
\text { cybersecurity breach in the past (note: } \\
\text { belief is sufficient). }\end{array}$ \\
\hline & & PriorExpImpact_Negative & $\begin{array}{l}\text { The result of experiencing a breach } \\
\text { in the past had a self-rated negative } \\
\text { effect on the respondent (e.g. doing less } \\
\text { online because of a past event, being less } \\
\text { confident). }\end{array}$ \\
\hline & & PriorExpImpact_Neutral & $\begin{array}{l}\text { The result of experiencing a breach in } \\
\text { the past had a self-rated neutral effect } \\
\text { on the respondent (e.g. no change in } \\
\text { behaviour or confidence). }\end{array}$ \\
\hline & & PriorExpImpact_Positive & $\begin{array}{l}\text { The result of experiencing a breach } \\
\text { in the past had a self-rated positive } \\
\text { effect on the respondent (e.g. increased } \\
\text { confidence, increased awareness, } \\
\text { increased skill). }\end{array}$ \\
\hline \multicolumn{4}{|c|}{ Privacy } \\
\hline & \multicolumn{2}{|c|}{ Privacy_Concerns } & General concerns about privacy online. \\
\hline & \multicolumn{2}{|c|}{ Privacy_ContentOwnership } & $\begin{array}{l}\text { Concerns about the ownership of } \\
\text { content that is put online. }\end{array}$ \\
\hline & \multicolumn{2}{|c|}{ PrivacyIntentionalDisclosure } & $\begin{array}{l}\text { Concerns about the intentional } \\
\text { disclosure of information to third parties } \\
\text { (often via a social networking service). }\end{array}$ \\
\hline & \multicolumn{2}{|c|}{ Privacy_Identity } & $\begin{array}{l}\text { Concerns arising from a person's online } \\
\text { identity. }\end{array}$ \\
\hline & & PrivacyID_FalseIdentity & $\begin{array}{l}\text { The use of a false identity for online } \\
\text { activities based on the perception that } \\
\text { this will safeguard the respondent's } \\
\text { privacy. }\end{array}$ \\
\hline & & PrivacyID_Truth & $\begin{array}{l}\text { Concerns identified by respondents } \\
\text { about telling the truth about yourself } \\
\text { online and the impact this may have on } \\
\text { your privacy and online identity. }\end{array}$ \\
\hline & \multicolumn{2}{|c|}{ Privacy_Protection } & $\begin{array}{l}\text { Concerns over protecting an individual's } \\
\text { privacy online. }\end{array}$ \\
\hline & \multicolumn{2}{|c|}{ Privacy_Tradeoff } & $\begin{array}{l}\text { The tradeoff of privacy for access to } \\
\text { materials or goods online. }\end{array}$ \\
\hline
\end{tabular}




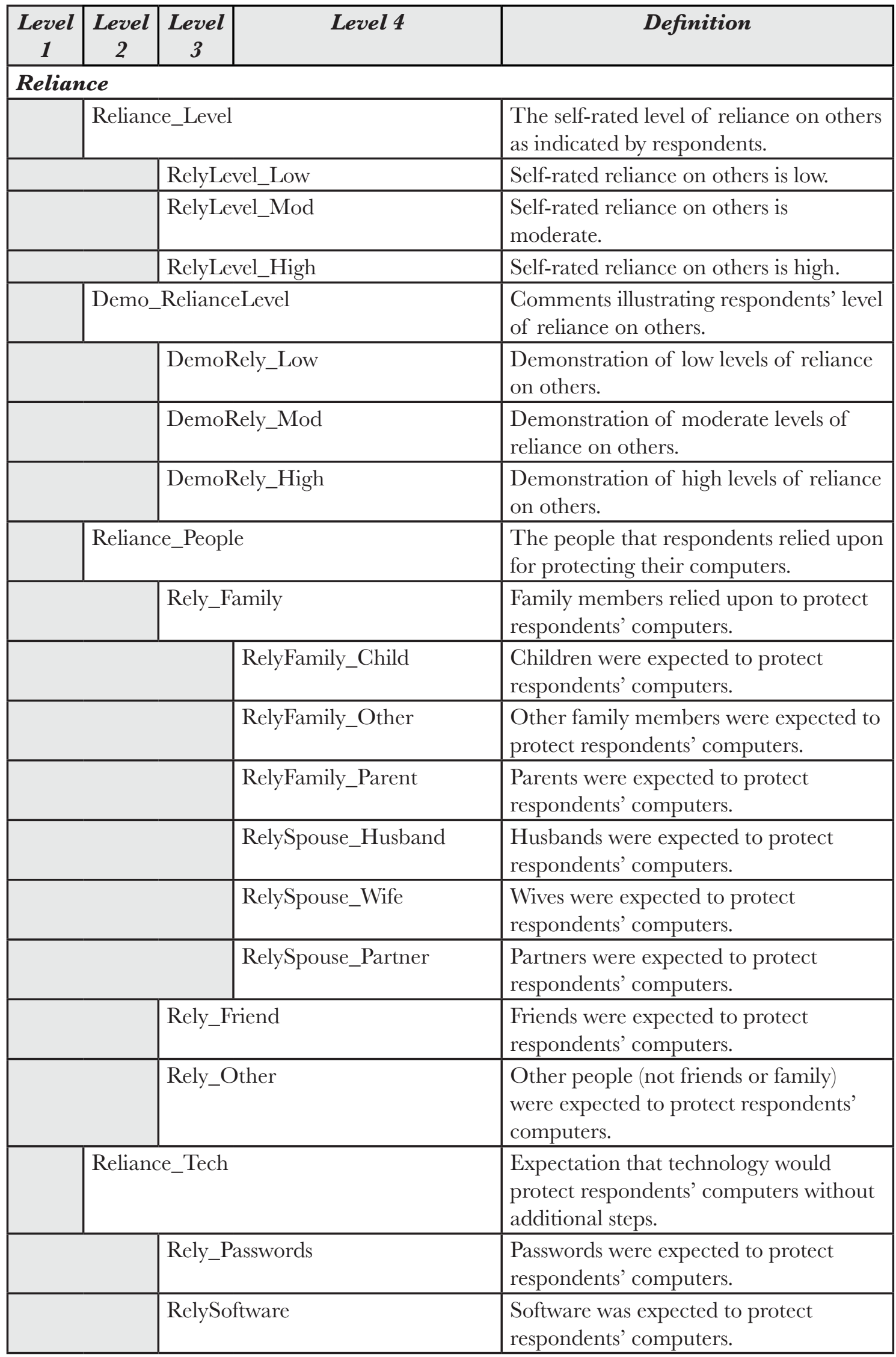




\begin{tabular}{|c|c|c|c|}
\hline $\begin{array}{c}\text { Level } \\
1\end{array}$ & \begin{tabular}{|c|} 
Level \\
2
\end{tabular} & \begin{tabular}{|c|} 
Level \\
3
\end{tabular} & Definition \\
\hline & & RelyWebsite & $\begin{array}{l}\text { Website owners were expected to be } \\
\text { responsible for ensuring that malware } \\
\text { was not hosted on their sites and would } \\
\text { not harm respondents' computers. }\end{array}$ \\
\hline \multicolumn{4}{|c|}{ Responsibility } \\
\hline & \multicolumn{2}{|c|}{ Responsibility_Bank } & $\begin{array}{l}\text { Belief that banks are responsible } \\
\text { for preventing and remedying the } \\
\text { consequences of cybersecurity breaches } \\
\text { (primarily financial). }\end{array}$ \\
\hline & \multicolumn{2}{|c|}{ Responsibility_Business } & $\begin{array}{l}\text { Belief that businesses are responsible } \\
\text { for preventing and remedying the } \\
\text { consequences of cybersecurity breaches. }\end{array}$ \\
\hline & \multicolumn{2}{|c|}{ Responsibility_Family } & $\begin{array}{l}\text { Belief that family members are } \\
\text { responsible for preventing and } \\
\text { remedying cybersecurity breaches. }\end{array}$ \\
\hline & & FamilyResp_Child & $\begin{array}{l}\text { Belief that respondents' children } \\
\text { are responsible for preventing and } \\
\text { remedying cybersecurity breaches. }\end{array}$ \\
\hline & & FamilyResp_Parent & $\begin{array}{l}\text { Belief that respondents' parents } \\
\text { are responsible for preventing and } \\
\text { remedying cybersecurity breaches. }\end{array}$ \\
\hline & & FamilyResp_Husband & $\begin{array}{l}\text { Belief that respondents' husbands } \\
\text { are responsible for preventing and } \\
\text { remedying cybersecurity breaches. }\end{array}$ \\
\hline & & FamilyResp_Wife & $\begin{array}{l}\text { Belief that respondents' wives are } \\
\text { responsible for preventing and } \\
\text { remedying cybersecurity breaches. }\end{array}$ \\
\hline & & FamilyRep_Partner & $\begin{array}{l}\text { Belief that respondent's partners } \\
\text { are responsible for preventing and } \\
\text { remedying cybersecurity breaches. }\end{array}$ \\
\hline & \multicolumn{2}{|c|}{ Responsibility_Other } & $\begin{array}{l}\text { Belief that someone else (not specified) } \\
\text { is responsible for preventing and } \\
\text { remedying cybersecurity breaches. }\end{array}$ \\
\hline & \multicolumn{2}{|c|}{ Responsibility_Personal } & $\begin{array}{l}\text { Belief that the respondent is responsible } \\
\text { for preventing and remedying the } \\
\text { consequences of cybersecurity breaches. }\end{array}$ \\
\hline & \multicolumn{2}{|c|}{ Responsibility_Telco } & $\begin{array}{l}\text { Belief that telecommunications } \\
\text { companies are responsible for preventing } \\
\text { and remedying the consequences of } \\
\text { cybersecurity breaches. }\end{array}$ \\
\hline & \multicolumn{2}{|c|}{ Responsibility_Website } & $\begin{array}{l}\text { Belief that website owners are } \\
\text { responsible for preventing and } \\
\text { remedying the consequences of } \\
\text { cybersecurity breaches that stem from } \\
\text { their sites. }\end{array}$ \\
\hline
\end{tabular}




\begin{tabular}{|c|c|c|c|c|}
\hline $\begin{array}{c}\text { Level } \\
1\end{array}$ & $\begin{array}{c}\text { Level } \\
2\end{array}$ & $\begin{array}{c}\text { Level } \\
3\end{array}$ & Level 4 & Definition \\
\hline & \multicolumn{3}{|c|}{ Responsibility_Work } & $\begin{array}{l}\text { Belief that respondents' employers } \\
\text { are responsible for preventing and } \\
\text { remedying the consequences of } \\
\text { cybersecurity breaches. }\end{array}$ \\
\hline \multicolumn{5}{|c|}{ Safeguards } \\
\hline & \multicolumn{3}{|c|}{ Safeguard_Behaviour } & $\begin{array}{l}\text { Behaviours that can be utilised in order } \\
\text { to safeguard an individual's computer. }\end{array}$ \\
\hline & & \multicolumn{2}{|c|}{ SafeBeh_Children } & $\begin{array}{l}\text { Safeguards taken in order to protect } \\
\text { children in the participant's household. }\end{array}$ \\
\hline & & & SafeChild_Discuss & $\begin{array}{l}\text { Discussing threats with children to make } \\
\text { them aware of the threats. }\end{array}$ \\
\hline & & & SafeChild_PublicPG & $\begin{array}{l}\text { Keeping the PG in a public place in the } \\
\text { home so that parents can monitor what } \\
\text { the child/ren are doing. }\end{array}$ \\
\hline & & \multicolumn{2}{|c|}{ SafeBeh_Data } & $\begin{array}{l}\text { Behaviours undertaken in order to } \\
\text { protect respondents' data. }\end{array}$ \\
\hline & & & DataSafe_HardCopies & $\begin{array}{l}\text { Keeping physical copies (rather than } \\
\text { only electronic copies) of important } \\
\text { data. }\end{array}$ \\
\hline & & & DataSafe_Redundancy & $\begin{array}{l}\text { Keeping a backup of important data } \\
\text { offsite. }\end{array}$ \\
\hline & & \multicolumn{2}{|c|}{ SafeBeh_Email } & $\begin{array}{l}\text { Safeguards associated with the use of } \\
\text { email. }\end{array}$ \\
\hline & & & EmailSafe_Attachments & $\begin{array}{l}\text { Not opening attachments if the sender } \\
\text { is not known or if the attachment isn't } \\
\text { expected. }\end{array}$ \\
\hline & & & EmailSafe_Suspicious & $\begin{array}{l}\text { Not opening emails where something in } \\
\text { the sender or subject is perceived to be } \\
\text { suspicious by the recipient. }\end{array}$ \\
\hline & & & EmailSafe_Unknown & $\begin{array}{l}\text { Not opening emails where the sender is } \\
\text { unknown to the recipient. }\end{array}$ \\
\hline & & \multicolumn{2}{|c|}{ SafeBeh_Other } & $\begin{array}{l}\text { Other behaviours associated with } \\
\text { cybersecurity. }\end{array}$ \\
\hline & & & OtherSafe_CheckAccts & $\begin{array}{l}\text { Regularly checking accounts or credit } \\
\text { cards that have been used or accessed } \\
\text { online in order to check for signs of } \\
\text { fraud. }\end{array}$ \\
\hline & & & OtherSafe_Cookies & $\begin{array}{l}\text { Checking the computer for new / } \\
\text { unwanted cookies. }\end{array}$ \\
\hline & & & OtherSafe_Trojans & $\begin{array}{l}\text { Checking downloads for Trojans prior to } \\
\text { installing anything. }\end{array}$ \\
\hline & & & OtherSafe_Disconnect & $\begin{array}{l}\text { Disconnecting from the internet when } \\
\text { not using the internet and only being } \\
\text { online when required. }\end{array}$ \\
\hline
\end{tabular}




\begin{tabular}{|c|c|c|c|c|}
\hline $\begin{array}{c}\text { Level } \\
1\end{array}$ & $\begin{array}{c}\text { Level } \\
2\end{array}$ & $\begin{array}{c}\text { Level } \\
3\end{array}$ & Level 4 & Definition \\
\hline & & & OtherSafe_Maintenance & $\begin{array}{l}\text { Undertaking general preventative } \\
\text { maintenance on the system including } \\
\text { backups, scans, etc. }\end{array}$ \\
\hline & & & OtherSafe_UpdatedSW & $\begin{array}{l}\text { Ensuring that all software being used is } \\
\text { up to date with any required patches or } \\
\text { updates. }\end{array}$ \\
\hline & & & OtherSafe_Careful & $\begin{array}{l}\text { General process of using safe } \\
\text { behaviours. }\end{array}$ \\
\hline & & & OtherSafe_Verisign & $\begin{array}{l}\text { Looking for and only entering personal } \\
\text { data on sites that utilise Verisign. }\end{array}$ \\
\hline & & SafeBe & A_Privacy & $\begin{array}{l}\text { Safeguard behaviours associated with } \\
\text { protecting users' privacy }\end{array}$ \\
\hline & & & PrivSafe_Careful & $\begin{array}{l}\text { Being careful about where personal } \\
\text { information is stored and what personal } \\
\text { information is provided to websites. }\end{array}$ \\
\hline & & & ProvSafe_ClearInfo & $\begin{array}{l}\text { Glearing any personal information from } \\
\text { websites before leaving them. }\end{array}$ \\
\hline & & & ProvSafe_ConsiderDisc & $\begin{array}{l}\text { Considering what would happen if } \\
\text { the information being stored on the } \\
\text { computer were to be disclosed in } \\
\text { deciding what should actually be stored } \\
\text { on the computer. }\end{array}$ \\
\hline & & & ProvSafe_SensitiveInfo & $\begin{array}{l}\text { Avoiding storing any personal } \\
\text { information on the computer including } \\
\text { personal documents. }\end{array}$ \\
\hline & & SafeBe & n_Web & $\begin{array}{l}\text { Safeguard behaviours taken in } \\
\text { association with using the internet and } \\
\text { general safeguard behaviours. }\end{array}$ \\
\hline & & & WebSafe_AwareDL & $\begin{array}{l}\text { Knowing what is being downloaded } \\
\text { and ensuring that nothing additional is } \\
\text { downloaded. }\end{array}$ \\
\hline & & & WebSafe_AwareSites & $\begin{array}{l}\text { Being aware of any signs that a website } \\
\text { may be malicious or likely to harm a } \\
\text { computer. }\end{array}$ \\
\hline & & & WebSafe_NoLinks & $\begin{array}{l}\text { Not clicking links in emails, on } \\
\text { webpages, in chat programs, or } \\
\text { elsewhere in order to prevent being } \\
\text { redirect to a website that is unsafe. }\end{array}$ \\
\hline & & & WebSafe_NoPopups & Not clicking on popup windows. \\
\hline & & & WebSafe_NoFinancial & $\begin{array}{l}\text { Decision not to use online financial } \\
\text { services such as online bill payments or } \\
\text { online banking. }\end{array}$ \\
\hline & & & WebSafe_SearchGoogle & $\begin{array}{l}\text { Searching any links that the user is } \\
\text { provided with on Google. }\end{array}$ \\
\hline
\end{tabular}




\begin{tabular}{|c|c|c|c|}
\hline $\begin{array}{c}\text { Level } \\
1\end{array}$ & $\begin{array}{c}\text { Level } \\
2\end{array}$ & $\begin{array}{c}\text { Level } \\
3\end{array}$ & Definition \\
\hline & \multicolumn{2}{|c|}{ Safeguard_Other } & $\begin{array}{l}\text { General safeguards that can be used to } \\
\text { protect a user's internet security that do } \\
\text { not fall into the other categories. }\end{array}$ \\
\hline & & OtherSafe_AdminControl & $\begin{array}{l}\text { Use of administrative controls such as } \\
\text { limiting account types or limiting use } \\
\text { of accounts in order to safeguard a } \\
\text { computer. }\end{array}$ \\
\hline & & OtherSafe_Backup & $\begin{array}{l}\text { Use of backups on a medium not stored } \\
\text { on the computer's hard drive in order to } \\
\text { create a copy of some or all of the user's } \\
\text { data. }\end{array}$ \\
\hline & & OtherSafe_LowLimitCG & $\begin{array}{l}\text { Use of a low limit credit card when } \\
\text { purchasing items online. }\end{array}$ \\
\hline & & OtherSafe_SecWireless & $\begin{array}{l}\text { Use of an encryption method on the } \\
\text { wireless networks used by the individual. }\end{array}$ \\
\hline & & OtherSafe_MultiFactor & $\begin{array}{l}\text { Use of multiple factor authentication } \\
\text { methods where possible (e.g. user name, } \\
\text { password, and authenticator code from } \\
\text { an app). }\end{array}$ \\
\hline & \multicolumn{2}{|c|}{ Safeguard_Password } & Safeguards associated with passwords. \\
\hline & & PassSafe_Change & $\begin{array}{l}\text { Users changing the the passwords they } \\
\text { use regularly }\end{array}$ \\
\hline & & PassSafe_NoWrite & $\begin{array}{l}\text { Not writing their passwords down where } \\
\text { others can see them. }\end{array}$ \\
\hline & & PassSafe_LongPW & $\begin{array}{l}\text { Actual use of long passwords whether or } \\
\text { not they are required by the site that the } \\
\text { password is for. }\end{array}$ \\
\hline & & PassSafe_Different & $\begin{array}{l}\text { Use of different passwords on different } \\
\text { sites by users. }\end{array}$ \\
\hline & & PassSafe_Code & $\begin{array}{l}\text { Writing a password down but using a } \\
\text { code in order to ensure that if someone } \\
\text { comes across it they cannot utilise it. }\end{array}$ \\
\hline & \multicolumn{2}{|c|}{ Safeguard_Software } & Safeguards based on protective software. \\
\hline & & SafeSoftware_AntiAdware & $\begin{array}{l}\text { Use of anti-adware software on an } \\
\text { individual's computer. }\end{array}$ \\
\hline & & SafeSoftware_AntiMalware & $\begin{array}{l}\text { Use of anti-malware software on an } \\
\text { individual's computer. }\end{array}$ \\
\hline & & SafeSoftware_AntiSpyware & $\begin{array}{l}\text { Use of anti-spyware software on an } \\
\text { individual's computer. }\end{array}$ \\
\hline & & SafeSoftware_AntiVirus & $\begin{array}{l}\text { Use of anti-virus software on an } \\
\text { individual's computer. }\end{array}$ \\
\hline & & SafeSoftware_Firewall & $\begin{array}{l}\text { Use of a firewall (whether separate or } \\
\text { built into the OS) on an individual's } \\
\text { computer. }\end{array}$ \\
\hline
\end{tabular}




\begin{tabular}{|c|c|c|c|c|}
\hline $\begin{array}{c}\text { Level } \\
1\end{array}$ & $\begin{array}{c}\text { Level } \\
2\end{array}$ & $\begin{array}{c}\text { Level } \\
3\end{array}$ & Level 4 & Definition \\
\hline & & \multicolumn{2}{|c|}{ SafeSoftware_PopupBlocker } & $\begin{array}{l}\text { Use of a specific pop-up blocker on an } \\
\text { individual's computer in order to block } \\
\text { popups on webpages. }\end{array}$ \\
\hline & \multicolumn{3}{|c|}{ Safeguard_Efficacy } & $\begin{array}{l}\text { Respondents' assessment about the } \\
\text { effectiveness of their safeguards at } \\
\text { preventing threats and mitigating their } \\
\text { consequences. }\end{array}$ \\
\hline & & \multicolumn{2}{|c|}{ SafeguardEfficacy_Low } & $\begin{array}{l}\text { The belief that the respondent's } \\
\text { safeguards have minimal effectiveness at } \\
\text { preventing cybersecurity breaches. }\end{array}$ \\
\hline & & \multicolumn{2}{|c|}{ SafeguardEfficacy_Mod } & $\begin{array}{l}\text { The belief that the respondent's } \\
\text { safeguards provide a moderate level of } \\
\text { effectiveness at preventing cybersecurity } \\
\text { breaches }\end{array}$ \\
\hline & & \multicolumn{2}{|c|}{ SafeguardEfficacy_High } & $\begin{array}{l}\text { The belief that the respondent's } \\
\text { safeguards are highly effective at } \\
\text { preventing cybersecurity breaches. }\end{array}$ \\
\hline & & \multicolumn{2}{|c|}{ SafeguardEfficacy_Unsure } & $\begin{array}{l}\text { Uncertainty by the respondent about } \\
\text { how effective the safeguards used are at } \\
\text { protecting against cybersecurity threats. }\end{array}$ \\
\hline & & \multicolumn{2}{|c|}{ SafeguardEfficacy_Inadequate } & $\begin{array}{l}\text { Refers to the perception that software is } \\
\text { not a totally adequate solution and that } \\
\text { there are some shortcomings }\end{array}$ \\
\hline \multicolumn{5}{|c|}{ ServicesUses } \\
\hline & \multicolumn{3}{|c|}{ Services } & $\begin{array}{l}\text { Online services that are used by } \\
\text { respondents (this specifically refers to } \\
\text { websites, both specific and general). }\end{array}$ \\
\hline & & \multicolumn{2}{|c|}{ Service_Bebo } & Bebo \\
\hline & & \multicolumn{2}{|c|}{ Service_Email } & $\begin{array}{l}\text { Email programs such as Outlook, Mac } \\
\text { Mail, and Thunderbird. }\end{array}$ \\
\hline & & \multicolumn{2}{|c|}{ ServiceFacebook } & Facebook \\
\hline & & \multicolumn{2}{|c|}{ Service_Games } & $\begin{array}{l}\text { Games (including downloadable games } \\
\text { that have an online component, are } \\
\text { downloaded from the internet, or are } \\
\text { played in a browser). }\end{array}$ \\
\hline & & \multicolumn{2}{|c|}{ Service_OnlineBanking } & $\begin{array}{l}\text { Online banking at the respondent's } \\
\text { financial institution. }\end{array}$ \\
\hline & & \multicolumn{2}{|c|}{ Service_Skype } & Skype \\
\hline & & \multicolumn{2}{|c|}{ Service_SocialNetworking } & $\begin{array}{l}\text { Other social networking sites aside from } \\
\text { Facebook and Bebo (inc. MySpace, } \\
\text { LinkedIn, etc) }\end{array}$ \\
\hline & & \multicolumn{2}{|c|}{ Service_Twitter } & Twitter \\
\hline
\end{tabular}




\begin{tabular}{|c|c|c|c|}
\hline $\begin{array}{c}\text { Level } \\
1\end{array}$ & $\begin{array}{c}\text { Level } \\
2\end{array}$ & $\begin{array}{c}\text { Level } \\
3\end{array}$ & Definition \\
\hline & & Service_Webmail & $\begin{array}{l}\text { Webmail services not using a separate } \\
\text { program but are instead accessed } \\
\text { via a browser either by choice or as } \\
\text { a requirement (e.g. Gmail, Yahoo, } \\
\text { Hotmail). }\end{array}$ \\
\hline & \multicolumn{2}{|l|}{ Uses } & $\begin{array}{l}\text { Uses of the internet that are not specific } \\
\text { to software. }\end{array}$ \\
\hline & & Use_Academic & $\begin{array}{l}\text { Academic uses such as research and } \\
\text { writing. }\end{array}$ \\
\hline & & Use_Browsing & $\begin{array}{l}\text { General browsing of websites for } \\
\text { information, leisure, etc. }\end{array}$ \\
\hline & & Use_Chat & $\begin{array}{l}\text { Synchronous communication via text } \\
\text { with other people (these individuals may } \\
\text { be known to the respondent in real life } \\
\text { or online only). }\end{array}$ \\
\hline & & Use_Communication & $\begin{array}{l}\text { Synchronous and asynchronous } \\
\text { communication with people online. }\end{array}$ \\
\hline & & Use_Music & $\begin{array}{l}\text { Listening to music online, typically via a } \\
\text { streaming service. }\end{array}$ \\
\hline & & Use_Blogs & Reading personal and corporate blogs \\
\hline & & Use_Research & $\begin{array}{l}\text { Researching information whether it is } \\
\text { for personal or academic uses. The goal } \\
\text { is to get an answer to a question that the } \\
\text { respondent has. }\end{array}$ \\
\hline & & Use_Shopping & Purchasing goods and services online. \\
\hline & & Use_Video & Watching videos, typically streaming. \\
\hline & & Use_FinancesOffline & $\begin{array}{l}\text { Although not an online activity, using } \\
\text { the computer to manage finances } \\
\text { by entering information from paper } \\
\text { statements into a spreadsheet that is } \\
\text { stored on the computer. }\end{array}$ \\
\hline & & Use_TravelBookings & $\begin{array}{l}\text { Making travel bookings (e.g. flights, } \\
\text { hotels, cars, events, etc.) }\end{array}$ \\
\hline \multicolumn{4}{|c|}{ Sharing } \\
\hline & \multicolumn{2}{|c|}{ Shared_NotShared } & $\begin{array}{l}\text { The computer is not shared with anyone } \\
\text { else in the house. }\end{array}$ \\
\hline & \multicolumn{2}{|c|}{ Shared_Family } & $\begin{array}{l}\text { The computer is shared with other } \\
\text { family members in the house (including } \\
\text { children). }\end{array}$ \\
\hline & \multicolumn{2}{|c|}{ Shared_Spouse } & $\begin{array}{l}\text { The computer is shared only with the } \\
\text { respondent's spouse or partner. }\end{array}$ \\
\hline & \multicolumn{2}{|c|}{ Shared_Other } & $\begin{array}{l}\text { The computer is shared with other } \\
\text { people outside of the respondent's } \\
\text { family). }\end{array}$ \\
\hline
\end{tabular}




\begin{tabular}{|c|c|c|c|c|}
\hline $\begin{array}{c}\text { Level } \\
1\end{array}$ & \begin{tabular}{|c|} 
Level \\
2
\end{tabular} & \begin{tabular}{|c|} 
Level \\
3
\end{tabular} & Level 4 & Definition \\
\hline \multicolumn{5}{|l|}{ Skill } \\
\hline & \multicolumn{3}{|c|}{ TechBackground } & $\begin{array}{l}\text { Whether or not the user has a technical } \\
\text { background either through work or } \\
\text { study }\end{array}$ \\
\hline & & \multicolumn{2}{|c|}{ TechBack_No } & $\begin{array}{l}\text { Respondents who do not have a } \\
\text { technical background through either } \\
\text { work or study. }\end{array}$ \\
\hline & & \multicolumn{2}{|c|}{ TechBack_Yes } & $\begin{array}{l}\text { Respondents who have a technical } \\
\text { background through either work or } \\
\text { study. }\end{array}$ \\
\hline & \multicolumn{3}{|c|}{ TechConfidence } & $\begin{array}{l}\text { Whether or not the respondent is } \\
\text { confident in their computer skills. }\end{array}$ \\
\hline & & \multicolumn{2}{|c|}{ TechConf_No } & $\begin{array}{l}\text { Respondents who are not confident in } \\
\text { their computer skills. }\end{array}$ \\
\hline & & \multicolumn{2}{|c|}{ TechConf_Yes } & $\begin{array}{l}\text { Respondents who are confident in their } \\
\text { computer skills. }\end{array}$ \\
\hline & \multicolumn{3}{|c|}{ TechSkill } & $\begin{array}{l}\text { Users' technical skills associated with } \\
\text { using a computer. }\end{array}$ \\
\hline & & \multicolumn{2}{|c|}{ TechSkill_No } & $\begin{array}{l}\text { Examples of a respondent } \\
\text { demonstrating little to no technical } \\
\text { skills associated with the use of their } \\
\text { computer. }\end{array}$ \\
\hline & & \multicolumn{2}{|c|}{ TechSkill_Yes } & $\begin{array}{l}\text { Examples of a respondent } \\
\text { demonstrating high levels of technical } \\
\text { skills associated with the use of their } \\
\text { computer. }\end{array}$ \\
\hline \multicolumn{5}{|c|}{ Threats } \\
\hline & \multicolumn{3}{|c|}{ Threat_ContentThreats } & $\begin{array}{l}\text { Threats where the threat comes from } \\
\text { the content itself (e.g. Spam). }\end{array}$ \\
\hline & & \multicolumn{2}{|c|}{ ContentThreat_Pornography } & $\begin{array}{l}\text { Perceived threats and consequences } \\
\text { associated with viewing pornography } \\
\text { online. }\end{array}$ \\
\hline & & \multicolumn{2}{|c|}{ ContentThreat_RestrictedContent } & $\begin{array}{l}\text { Perceived threats and consequences } \\
\text { associated with viewing content that is } \\
\text { inappropriate for certain ages, illegal, } \\
\text { etc. }\end{array}$ \\
\hline & \multicolumn{3}{|c|}{ Threat_EmailThreats } & Threats that are contained in email \\
\hline & & \multicolumn{2}{|c|}{ EmailThreat_Fraud } & $\begin{array}{l}\text { Emails that are sent fraudulently (e.g. a } \\
\text { person posing as another person, or a } \\
\text { person posing as you sending emails) }\end{array}$ \\
\hline & & \multicolumn{2}{|c|}{ EmailThreat_Phishing } & $\begin{array}{l}\text { Emails that are sent posing as an } \\
\text { organisation (or person) in order to } \\
\text { obtain money or account information. }\end{array}$ \\
\hline
\end{tabular}




\begin{tabular}{|c|c|c|c|c|}
\hline $\begin{array}{c}\text { Level } \\
1\end{array}$ & $\begin{array}{c}\text { Level } \\
2\end{array}$ & $\begin{array}{c}\text { Level } \\
3\end{array}$ & Level 4 & Definition \\
\hline & & \multicolumn{2}{|c|}{ EmailThreat_Spam } & $\begin{array}{l}\text { Mass emails that are sent without the } \\
\text { respondent having requested the email. }\end{array}$ \\
\hline & \multicolumn{3}{|c|}{ Threat_MalwareThreats } & $\begin{array}{l}\begin{array}{l}\text { Threats that are part of malicious } \\
\text { software }\end{array} \\
\end{array}$ \\
\hline & & \multicolumn{2}{|c|}{ MalwareThreat_Malware } & Malware (very general use of the term). \\
\hline & & \multicolumn{2}{|c|}{ MalwareThreat_Spyware } & $\begin{array}{l}\text { Spyware (a type of malicious software } \\
\text { that gathers information about a person } \\
\text { without their knowledge and sends it to } \\
\text { a third party. This may include online } \\
\text { behaviours, passwords, user names, etc.). }\end{array}$ \\
\hline & & \multicolumn{2}{|c|}{ MalwareThreat_Trojan } & $\begin{array}{l}\text { Trojan Horses (a type of malware where } \\
\text { the user is lead to believe that a program } \\
\text { is meant to do one thing when in reality } \\
\text { it also does something malicious). }\end{array}$ \\
\hline & & \multicolumn{2}{|c|}{ MalwareThreat_Virus } & $\begin{array}{l}\text { Viruses (a type of malware that requires } \\
\text { another program for it to work). }\end{array}$ \\
\hline & & \multicolumn{2}{|c|}{ MalwareThreat_Worm } & $\begin{array}{l}\text { Worms (a type of malware that does not } \\
\text { require another program for it to work). }\end{array}$ \\
\hline & \multicolumn{3}{|c|}{ Threat_OfflineThreats } & $\begin{array}{l}\text { Threats that take place offline, but } \\
\text { that users mentioned in the context of } \\
\text { cybersecurity. }\end{array}$ \\
\hline & & \multicolumn{2}{|c|}{ OfflineThreat_CGFraud } & $\begin{array}{l}\text { Credit card fraud that does not } \\
\text { necessarily have to take place online }\end{array}$ \\
\hline & & \multicolumn{2}{|c|}{ OfflineThreat_PrivateInfoPublic } & $\begin{array}{l}\text { Any private data being made public, } \\
\text { though not necessarily through a typical } \\
\text { cybersecurity context. }\end{array}$ \\
\hline & & \multicolumn{2}{|c|}{ OfflineThreat_CompStolen } & $\begin{array}{l}\text { Information being made public or } \\
\text { sensitive information being stolen due to } \\
\text { a stolen device. }\end{array}$ \\
\hline & \multicolumn{3}{|c|}{ Threat_OtherThreats } & $\begin{array}{l}\text { Other threats to respondents' } \\
\text { cybersecurity. }\end{array}$ \\
\hline & & \multicolumn{2}{|c|}{ OtherThreat_NigerianScam } & $\begin{array}{l}\text { Communications that are made } \\
\text { suggesting that a large amount of money } \\
\text { will be deposited in the recipient's } \\
\text { bank account if they cooperate with } \\
\text { the sender who is purported to be } \\
\text { working on behalf of a prominent } \\
\text { figure. Although these scams are often } \\
\text { referred to as Nigerian Prince scams, the } \\
\text { purported source of the money may be } \\
\text { different. }\end{array}$ \\
\hline & \multicolumn{3}{|c|}{ OtherThreat_Scams } & $\begin{array}{l}\text { General scams (without specifying how } \\
\text { they are distributed) that respondents } \\
\text { need to be concerned about online. }\end{array}$ \\
\hline & \multicolumn{3}{|c|}{ Threat_WebThreats } & The threat source is a web page. \\
\hline
\end{tabular}




\begin{tabular}{|c|c|c|c|c|}
\hline $\begin{array}{c}\text { Level } \\
1\end{array}$ & $\begin{array}{c}\text { Level } \\
2\end{array}$ & $\begin{array}{c}\text { Level } \\
3\end{array}$ & Level 4 & Definition \\
\hline & & WebT & eignCookies & $\begin{array}{l}\text { Cookies are placed on a user's system } \\
\text { that are not from the site(s) that the user } \\
\text { visited. }\end{array}$ \\
\hline & & WebTl & IWebsites & Websites that host malicious content. \\
\hline & & WebTl & Irming & $\begin{array}{l}\text { Webpages that are meant to look like } \\
\text { non-malicious pages and that often copy } \\
\text { non-malicious pages in order to get } \\
\text { users to enter personal information on } \\
\text { them (e.g. fake banking pages). }\end{array}$ \\
\hline & & WebTl & ups & $\begin{array}{l}\text { Small windows that appear when a site } \\
\text { is visited (the popups that are being } \\
\text { referred to here are unwanted popups, } \\
\text { such as advertising, that are not the } \\
\text { result of an individual intentionally } \\
\text { clicking something that should generate } \\
\text { a new window). }\end{array}$ \\
\hline & Threat & -Threat & & $\begin{array}{l}\text { Respondents' beliefs of where threats } \\
\text { come from. }\end{array}$ \\
\hline & & Threat & accident & Threat was caused by an accident. \\
\hline & & Threat & & $\begin{array}{l}\text { Belief that some ISPs are more prone to } \\
\text { threats than other ISPs due to different } \\
\text { spam / content filtering. }\end{array}$ \\
\hline & & Threat & thatrooms & $\begin{array}{l}\text { Belief that synchronous online } \\
\text { chatrooms are a source of online threats. }\end{array}$ \\
\hline & & Threat & ownloads & $\begin{array}{l}\text { Belief that downloads of software, } \\
\text { music, and videos can contain } \\
\text { cybersecurity threats. }\end{array}$ \\
\hline & & Threat & Cards & $\begin{array}{l}\text { Malicious software disguised as } \\
\text { electronic cards. }\end{array}$ \\
\hline & & Threat & xpiredSoftware & $\begin{array}{l}\text { Threats being caused by expired security } \\
\text { software not catching the threats. }\end{array}$ \\
\hline & & Threat & inks & $\begin{array}{l}\text { Perception that sites/emails/chat } \\
\text { messages/social networking pages may } \\
\text { link to other sites that are malicious. }\end{array}$ \\
\hline & & Threat & Vebsites & $\begin{array}{l}\text { Threats of private information being } \\
\text { disclosed because a website is not } \\
\text { secured. }\end{array}$ \\
\hline & & Threat & ocialNetworking & $\begin{array}{l}\text { Perception that social networking } \\
\text { can be a source of online threats } \\
\text { through disclosure of information or } \\
\text { downloading of malicious software. }\end{array}$ \\
\hline & & Threat & ersistentLogin & $\begin{array}{l}\text { Belief that staying logged on to a } \\
\text { website can be a threat (e.g. frapeing) }\end{array}$ \\
\hline
\end{tabular}




\begin{tabular}{|c|c|c|c|}
\hline $\begin{array}{c}\text { Level } \\
1\end{array}$ & \begin{tabular}{|c|} 
Level \\
2 \\
\end{tabular} & $\begin{array}{c}\text { Level } \\
3\end{array}$ & Definition \\
\hline \multicolumn{4}{|c|}{ Trust } \\
\hline & \multicolumn{2}{|c|}{ Trust_Trusted } & $\begin{array}{l}\text { People and things that respondents } \\
\text { consider to be trustworthy. }\end{array}$ \\
\hline & & Trust_DirectLinks & $\begin{array}{l}\text { Links that are provided directly from a } \\
\text { website to another part of a website. }\end{array}$ \\
\hline & & Trust_Google & Links from Google are trustworthy. \\
\hline & & Trust_OnlineInfo & Information that has been found online. \\
\hline & & Trust_People & $\begin{array}{l}\text { People that respondents interact with } \\
\text { online (e.g. auction buyers / sellers, } \\
\text { people on social networks, etc.). }\end{array}$ \\
\hline & & Trust_InherentTrust & $\begin{array}{l}\text { Certain websites are considered to be } \\
\text { inherently trustworthy because of their } \\
\text { nature and reputation. }\end{array}$ \\
\hline & \multicolumn{2}{|c|}{ Trust_Distrusted } & $\begin{array}{l}\text { People or things that respondents } \\
\text { consider to be untrustworthy. }\end{array}$ \\
\hline & & Distrust_GoogleLinks & Links from Google are untrustworthy. \\
\hline \multicolumn{4}{|c|}{ UserMotivations } \\
\hline & \multicolumn{2}{|c|}{ Motive_AvoidDataLoss } & $\begin{array}{l}\text { The reason for engaging in the indicated } \\
\text { safeguards is to avoid data loss from } \\
\text { happening. }\end{array}$ \\
\hline & \multicolumn{2}{|c|}{ Motive_AvoidVulnerability } & $\begin{array}{l}\text { The reason for engaging in the indicated } \\
\text { safeguards is to avoid feeling vulnerable } \\
\text { to the consequences of cybersecurity } \\
\text { breaches. }\end{array}$ \\
\hline & \multicolumn{2}{|c|}{ Motive_AvoidTimeConseq } & $\begin{array}{l}\text { The reason for engaging in the } \\
\text { indicated safeguards is to avoid losing } \\
\text { time associated with remedying the } \\
\text { consequences of a cybersecurity breach } \\
\text { (e.g. restoring from backup, installing } \\
\text { new software, etc). }\end{array}$ \\
\hline & \multicolumn{2}{|c|}{ Motive_BusRequirement } & $\begin{array}{l}\text { The reason for engaging in the indicated } \\
\text { safeguards it because it is a requirement } \\
\text { associated with the respondent's job that } \\
\text { they act in a certain way or maintain } \\
\text { a certain level of security on their } \\
\text { personal computers. }\end{array}$ \\
\hline & \multicolumn{2}{|c|}{ Motive_GeneralProtect } & $\begin{array}{l}\text { The reason for engaging in the indicated } \\
\text { safeguards is so that the person feels that } \\
\text { they have a general level of protection. }\end{array}$ \\
\hline & \multicolumn{2}{|c|}{ Motive_MitigateRisks } & $\begin{array}{l}\text { The reason for engaging in the indicated } \\
\text { safeguards is to mitigate the effects of } \\
\text { risks (vague). }\end{array}$ \\
\hline
\end{tabular}




\begin{tabular}{|c|c|c|c|c|}
\hline $\begin{array}{c}\text { Level } \\
1\end{array}$ & \begin{tabular}{|c|} 
Level \\
2
\end{tabular} & $\begin{array}{c}\text { Level } \\
3\end{array}$ & Level 4 & Definition \\
\hline & \multicolumn{3}{|c|}{ Motive_FreeTrial } & $\begin{array}{l}\text { The reason for engaging in the indicated } \\
\text { safeguards is because a free trial of } \\
\text { protective software had a positive effect } \\
\text { on the respondents during the trial stage. }\end{array}$ \\
\hline & \multicolumn{3}{|c|}{ Motive_PreventBadBeh } & $\begin{array}{l}\text { The reason for engaging in the indicated } \\
\text { safeguards is to prevent bad behaviours } \\
\text { of other people using the computer } \\
\text { from impacting on the respondent. }\end{array}$ \\
\hline & \multicolumn{3}{|c|}{ Motive_PriorExp } & $\begin{array}{l}\text { The reason for engaging in the indicated } \\
\text { safeguards is because of the past } \\
\text { experience that the respondent had with } \\
\text { a cybersecurity breach (can be positive } \\
\text { or negative). }\end{array}$ \\
\hline & \multicolumn{3}{|c|}{ Motive_ProtectKids } & $\begin{array}{l}\text { The reason for engaging in the indicated } \\
\text { safeguards is to protect the respondent's } \\
\text { child/ren. }\end{array}$ \\
\hline & \multicolumn{3}{|c|}{ Motive_ThinkLikeMalUser } & $\begin{array}{l}\text { The reason for engaging in the indicated } \\
\text { safeguards is because the respondent } \\
\text { has put themselves in the shoes of a } \\
\text { malicious user and wants to take the } \\
\text { steps they believe will protect against } \\
\text { how they see the malicious user acting. }\end{array}$ \\
\hline \multicolumn{5}{|l|}{ Value } \\
\hline & \multicolumn{3}{|c|}{ Value_Data } & $\begin{array}{l}\text { Respondents' self-assessed perceptions } \\
\text { of how valuable the data contained on } \\
\text { their computer was. }\end{array}$ \\
\hline & & \multicolumn{2}{|c|}{ DataValue_Low } & $\begin{array}{l}\text { Perception that the data contained on } \\
\text { the respondent's computer was of low } \\
\text { value. }\end{array}$ \\
\hline & & \multicolumn{2}{|c|}{ DataValue_Mod } & $\begin{array}{l}\text { Perception that the data contained } \\
\text { on the respondent's computer was of } \\
\text { moderate value. This meant that some } \\
\text { information was considered valuable } \\
\text { while other information was not. }\end{array}$ \\
\hline & & \multicolumn{2}{|c|}{ DataValue_High } & $\begin{array}{l}\text { Perception that the data contained on } \\
\text { the respondent's computer was of high } \\
\text { value. }\end{array}$ \\
\hline & \multicolumn{3}{|c|}{ Value_Criteria } & $\begin{array}{l}\text { Criteria identified by respondents about } \\
\text { what made data more or less valuable. }\end{array}$ \\
\hline & & \multicolumn{2}{|c|}{ ValueCrit_Content } & $\begin{array}{l}\text { Type of content that could be affected } \\
\text { by a cybersecurity breach. }\end{array}$ \\
\hline & & & ValueContent_Photos & $\begin{array}{l}\text { Personal photos are considered as a } \\
\text { valuable type of data. }\end{array}$ \\
\hline & & & ValueContent_Videos & $\begin{array}{l}\text { Personal videos are considered as a } \\
\text { valuable type of data. }\end{array}$ \\
\hline
\end{tabular}




\begin{tabular}{|c|c|c|c|c|}
\hline $\begin{array}{c}\text { Level } \\
1\end{array}$ & $\begin{array}{c}\text { Level } \\
2\end{array}$ & $\begin{array}{c}\text { Level } \\
3\end{array}$ & Level 4 & Definition \\
\hline & & & ValueContent_Purchases & $\begin{array}{l}\text { Purchased music and videos are } \\
\text { considered as a valuable type of data. }\end{array}$ \\
\hline & & & ValueContent_WorkSchool & $\begin{array}{l}\text { Documents created for work or school } \\
\text { are considered as a valuable type of } \\
\text { data. }\end{array}$ \\
\hline & & & ValueContent_Comm & $\begin{array}{l}\text { Communications between individuals } \\
\text { are considered as a valuable type of } \\
\text { data. }\end{array}$ \\
\hline & & \multicolumn{2}{|c|}{ ValueCrit_Replace } & $\begin{array}{l}\text { Whether or not the the data could be } \\
\text { easily replaced either through a backup } \\
\text { or through other means. }\end{array}$ \\
\hline & & \multicolumn{2}{|c|}{ ValueCrit_Use } & $\begin{array}{l}\text { The way that the computer is used and } \\
\text { the relationship to the value of data } \\
\text { stored on it. }\end{array}$ \\
\hline \multicolumn{5}{|c|}{ WorkImpact } \\
\hline & \multicolumn{3}{|c|}{ WorkImpact_Awareness } & $\begin{array}{l}\text { Belief by the user that through the } \\
\text { course of their work, they become more } \\
\text { aware of the threats that they may be } \\
\text { faced with (however, the awareness is not } \\
\text { required as part of their work). }\end{array}$ \\
\hline & \multicolumn{3}{|c|}{ WorkImpact_Knowledge } & $\begin{array}{l}\text { Belief by the user that through the } \\
\text { course of their work they become more } \\
\text { knowledgeable about how threats work. }\end{array}$ \\
\hline & \multicolumn{3}{|c|}{ WorkImpact_Requirement } & $\begin{array}{l}\text { Belief by the user that they are more } \\
\text { aware of security at home because they } \\
\text { are required to be aware of it at work. }\end{array}$ \\
\hline & \multicolumn{3}{|c|}{ WorkImpact_Sector } & $\begin{array}{l}\text { Belief by the user that they are more } \\
\text { aware of security at home because of } \\
\text { the job sector that they work in. }\end{array}$ \\
\hline & \multicolumn{3}{|c|}{ WorkImpact_WorkBetter } & $\begin{array}{l}\text { Belief that the user's workplace is better } \\
\text { at protecting a work computer than } \\
\text { the user is at protecting their home } \\
\text { computerRefers to the impact of work } \\
\text { requiring security on their employee's } \\
\text { home computer, even for personal } \\
\text { browsing. }\end{array}$ \\
\hline & \multicolumn{3}{|c|}{ WorkImpact_HomeRequirements } & $\begin{array}{l}\text { Impact of work requiring security on } \\
\text { their employee's home computer, even } \\
\text { for personal browsing. }\end{array}$ \\
\hline
\end{tabular}


Braun 


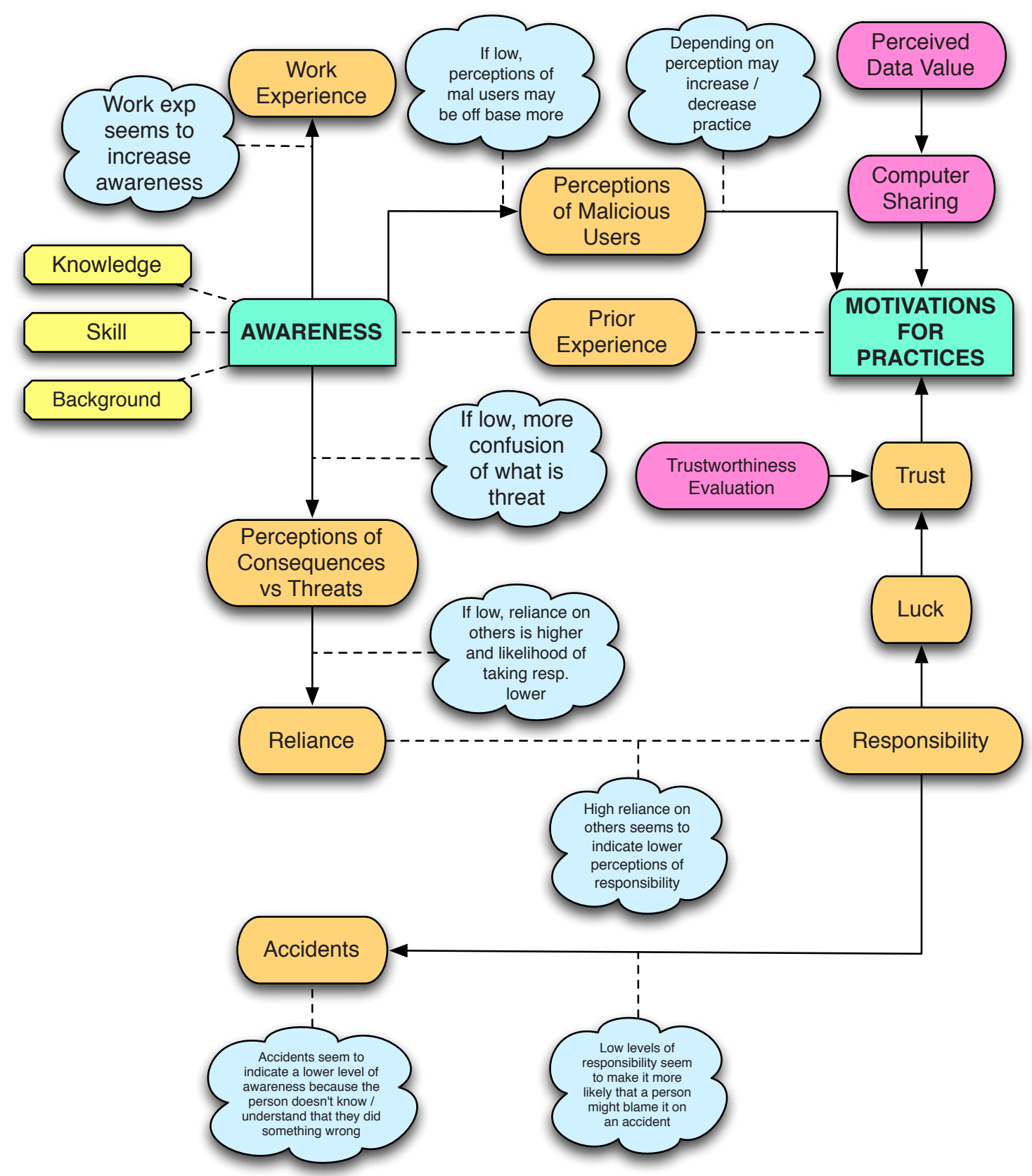

Figure A-1 Mind map of initial relationships between codes from focus groups and individual interviews 
Braun 


\section{Appendix 6 Information sheet \& consent form}

\section{Information Sheet}

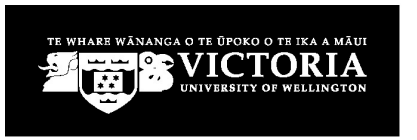

SCHOOL OF INFORMATION MANAGEMENT

TE KURA TIAKI, WHAKAWHITI KŐRERO

LEVEL 5, RUTHERFORD HOUSE, PIPITEA CAMPUS, 23 LAMBTON QUAY, WELLINGTON

PO Box 600, Wellington 6140, New Zealand

Phone + 64-4-4635103 Fax +64-4-4635446 Email sim@vuw.ac.nz Website www.victoria.ac.nz/sim

Project Title: Cybersecurity Awareness and Implementation: Factors influencing user behaviours relating to awareness of threats and use of safeguards

Researcher: Nicole K Henika Braun

I am a PhD student in the School of Information Management at Victoria University of Wellington. As part of my degree I am undertaking a research project which will lead to the production of a thesis. This research is examining home users' internet security awareness and behaviours.

\section{Research Goals}

The goals of this research are to (1) identify the factors affecting home user awareness of cybersecurity threats and safeguards; (2) identify home user cybersecurity practices; and (3) determine how user awareness of threats and safeguards can impact users' cybersecurity practices. By achieving these research goals, it will increase the amount of knowledge that we have about what users do to protect themselves online and why they engage in various cybersecurity behaviours. In the future this knowledge may help to develop better internet security software and improve the way that we teach people how to behave safely online. In order to carry out this research, the University requires ethical approval to be obtained.

\section{Focus Group Sessions}

My research will consist of two parts: (1) focus groups and (2) interviews. In the first phase I will be conducting three focus groups. The focus groups will take place in the latter part of 2009. In 2010 I will be conducting individual interviews based on the findings of the focus groups. 
SCHOOL OF INFORMATION MANAGEMENT

TE KURA TIAKI, WHAKAWHITI KŌRERO

LEVEL 5, RUTHERFORD HOUSE, PIPITEA CAMPUS, 23 LAMBTON QUAY, WELLINGTON

PO Box 600, Wellington 6140, New Zealand

Phone +64-4-4635103 Fax +64-4-4635446 Email sim@vuw.ac.nz Website www.victoria.ac.nz/sim

I am currently seeking participants for a focus group. I am looking for individuals who use a computer with an internet connection at home and who primarily use this computer for personal use. In this research, a computer is considered for personal use if: (1) the majority of the tasks that you do on the computer are related to personal rather than work activities and (2) the computer is owned and controlled by you or a member of your family rather than by your employer.

If you choose to participate in this research, you will be part of a group along with 5-7 other participants where you will have the opportunity to discuss your views and experiences relating to Internet security. It is expected that the focus group discussion will last approximately 1.5 hours.

Each focus group will be recorded on video for use in analysing the data. Using a video recording will allow me to identify who is speaking and to examine body language and non-verbal responses that take place during the discussion which may be useful during the data analysis phase.

\section{Individual Interviews}

My research consists of 2 parts. The first part, focus group discussions, took place in late 2009. I am now conducting individual interviews based on the findings of the focus groups.

I am currently seeking participants for individual interviews who use a computer with an internet connection at home and who primarily use this computer for personal use. In this research, a computer is considered for personal use if: (1) the majority of the tasks that you do on the computer are related to personal rather than work activities and (2) the computer is owned and controlled by you or a member of your family rather than by your employer. 
Appendix 6

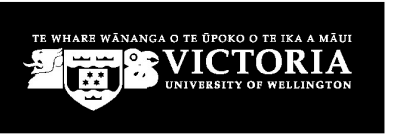

SCHOOL OF INFORMATION MANAGEMENT

TE KURA TIAKI, WHAKAWHITI KŐRERO

LEVEL 5, RUTHERFORD HOUSE, PIPITEA CAMPUS, 23 LAMBTON QUAY, WELLINGTON

PO Box 600, Wellington 6140, New Zealand

Phone + 64-4-4635103 Fax +64-4-4635446 Email sim@vuw.ac.nz Website www.victoria.ac.nz/sim

If you choose to participate in this research, you will have the opportunity to discuss your views on internet security with me. It is expected that the interview will last for approximately 1 hour. Each interview will be recorded on a digital audio recorder and transcribed by me. After the interview has been transcribed, this transcription will be made available to you for verification.

\section{Confidentiality}

All responses will be confidential and will only be reported in an aggregated way or in a way where individuals and their associated groups will not be able to be identified. In order to protect the privacy of all participants in the group, as part of the focus group it is essential that the discussions that take place during the session are kept confidential by all participants.

All electronic data will be kept in a password protected file. Any written material collected will be kept in a locked cabinet. All material will be kept confidential, and will only be seen by me, and my supervisors, Dr. Dan Dorner and Dr. Val Hooper. The thesis will be submitted for marking to the School of Information Management and deposited in the University Library and the university's digital repository. It is also intended that one or more articles using the data will be submitted for publication in scholarly journals. All raw data from the focus groups, including video tapes and video files, will be destroyed three years after the end of the project.

\section{Withdrawal from Study}

Because of the group discussions that take place in focus groups, you may only withdraw from participating prior to the start of the focus group session. 


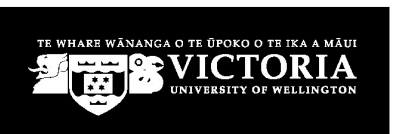

SCHOOL OF INFORMATION MANAGEMENT

TE KURA TIAKI, WHAKAWHITI KŐRERO

LEVEL 5, RUTHERFORD HOUSE, PIPITEA CAMPUS, 23 LAMBTON QUAY, WELLINGTON

PO Box 600, Wellington 6140, New Zealand

Phone + 64-4-4635103 Fax +64-4-4635446 Email sim@vuw.ac.nz Website www.victoria.ac.nz/sim

\section{Contact}

A summary of the focus group results will be available after the data from all of the focus groups have been analysed. If you wish to receive this summary, please indicate so on the consent form and provide an email address where it can be sent.

If you have any questions or would like further information about the project, please contact me or one of my supervisors:
Nicole Braun
nicole.braun@vuw.ac.nz
(04) 463-8911
Dr. Dan Dorner
dan.dorner@vuw.ac.nz
(04) 463-5781
Dr. Val Hooper
val.hooper@vuw.ac.nz
(04) 463-5020

Attached, you will find a consent form, a request for contact details, and an overview of the main topics we will discuss during the focus group. If you agree to participate in the focus group, carefully read the consent form before signing it and returning it to me along with the completed contact details form in the stamped envelope provided. I will be in touch again to verify a suitable time for the focus group session.

Thank you,

Nicole K Henika Braun 


\section{Consent Form}

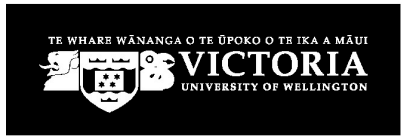

SCHOOL OF INFORMATION MANAGEMENT

TE KURA TIAKI, WHAKAWHITI KÖRERO

LEVEL 5, RUTHERFORD HOUSE, PIPITEA CAMPUS, 23 LAMBTON QUAY, WELLINGTON

PO Box 600, Wellington 6140, New Zealand

Phone + 64-4-4635103 Fax +64-4-4635446 Email sim@vuw.ac.nz Website www.victoria.ac.nz/sim

Please indicate your acceptance of each term by placing a tick mark in the box next to the statement.

I have been provided with adequate information relating to the nature and objectives of this research project. I have understood that information and have been given the opportunity to seek further clarification or explanations.

I understand that the focus groups will be video recorded.

I understand that any information or opinions I provide will be kept confidential and reported only in an aggregated / non-attributable form.

I understand that I must keep the discussions of the focus group confidential and may not disclose what others have said during the focus group session. [focus gorups only]

I understand that the information I have provided will be used only for the purposes stated in the information sheet and will not be released to others without my written consent.

I understand that when this research is completed the information obtained will be destroyed after 3 years after the completion of the project.

I would like to receive a summary of the results of the focus groups after all focus groups have been completed [focus groups only].

Please provide an email address:

I would like to receive a copy of the transcript from the interview for checking [individual interviews only].

Please provide an email address:

I agree to take part in this research.

I understand that I may only withdraw from participating in the focus group before the session starts [focus groups only]. 
Braun 


\section{Appendix $7 \quad$ Explanation of ranking used in the coding process}

Respondents specifically and implicitly indicated low to high presence of specific factors, in particular:

- threat awareness,

- safeguard awareness,

- knowledge,

- perceived self-efficacy,

- trust,

- reliance,

- prior experience, and

- security orientation

In Chapters 4 and 6, these measures were useful to help explain how the different factors affected individuals. Although a five-point measure was used to explain respondents features, the categories of low-moderate and moderate-high were used to explain the collective group of respondents where some had slightly lower or higher levels of the factors present. This allowed for some flexibility amongst the identification of the users while still presenting a cohesive group of individuals based on their features.

For certain factors (threat awareness, safeguard awareness, knowledge, and perceived self-efficacy), respondents provided clear indications of high, average, and low in their responses. In some cases, respondents indicated that they were "slightly above average" or "slightly below average." Responses where individuals explained that they were "slightly above average" or made other similar comments were rated as "moderate to high," while "slightly below average" and similar comments were rated as "low to moderate."

In order to provide a greater explanation of how the codes were assigned (low, moderate, high) for the remaining factors, the process for assigning the codes to reliance will be explained in greater detail. Reliance, prior experience, and security orientation were the main factors where respondents did not explicitly indicate high or low levels.

Although initial comments about responsibility suggested that respondents felt responsible for their own security, further discussions showed that these feelings of responsibility were not always genuine. As a result, assigning ranks of low, moderate, and high to respondents comments about their reliance on others required additional interpretation to assign these values.

Comments associated with low levels of reliance typically included comments about how the individual could not rely on anyone else. For example: 
- You [other people] rely too much on software you bought that might not update for 15 or 20 days when you should be updating all the time.

- [When explaining why he did not rely on other people to help keep him informed about security] I think that people around me are less aware of the security threats as I do, as I am.

In each of these cases, the respondents comments indicated a level of uncertainty associated with security that they was attached to relying on someone or something else to keep them protected from threats. Therefore, in order to eliminate their uncertainty, they expressed that they did not want to rely on other people to help keep their computers safe online. As a result, one of the key things that was associated with low levels of reliance was a desire to avoid the uncertainty that other people could introduce if the respondent looked outside of their own knowledge and skills to protect themselves.

High levels of reliance were typically associated with comments about a lack of knowledge and awareness about threats and safeguards, but an understanding about the importance of security. These two features were the first indications that higher levels of reliance could be present. The highest levels of reliance were associated with respondents who did not actively take any security steps themselves. Instead, they often relied on someone else, usually a family member, to take case of their computer security for them. For example:

- I have a husband who is a network engineer who looks after the things like making sure we've got antivirus software installed and that it's sort of automatically updating itself

- I don't know how to do that protection, but I would be aware that you need to.

- I rely on [my husband], her dad, to be our technician at home. "How do I do this?"

In these cases, there was an awareness of the need for security, but a typically a lack of understanding about how to carry those behaviours out. As a result, respondents with higher levels of reliance typically depended on someone that they believed had much higher levels of skills than they did. In many cases, respondents with high levels of reliance 
were clear that they expected someone else to carry out the necessary steps to protect their home computer because they did not believe that they could do these steps themselves.

Moderate levels of reliance were associated with comments about the steps that the respondents took themselves along with indications that they placed some level of reliance on someone or something to either prevent or remedy the effects of cybersecurity breaches. One example of this is respondents who took steps to protect themselves (such as using antivirus software or firewall software) but who expected banks or other websites to remedy issues if they were to occur. For example, the below comments were made by people who took steps to proactively protect their security, but who would expect someone else to remedy issues:

- They're the experts [the security companies], and I'm relying on them to keep my machine clean and free of viruses. And if it does get infected, clean it.

- When talking about why he was not concerned about financial consequences of cybersecurity breaches]...if something did occur, credit card companies will reimburse you if you file right away, which I would.

In these comments there was a common belief that a company would remedy the consequences of a breach. However, these respondents still ensured that they maintained some responsibility for protecting their computers in the first place, even if that protection was as simple as installing protective software. Another example of more moderate levels of reliance is when respondents took steps to protect themselves, but expected a family member to provide information about threats or to fix problems that arose, limiting how much responsibility they took in the event of a cybersecurity breach. For example:

- I would ask my husband first because he's quite knowledgeable, and I know he is. And then I would probably go and look on the internet.

- I'd probably check with our, with one of my kids, with the feeling that they would maybe [pause] especially our older one, I'd expect he'd have a little more knowledge or say "oh, mom, don't worry it"

In both cases, the respondents relied on someone else in the family to provide them with information about security. While these respondents would seek out some information, 
there was a greater reliance on other people rather than proactively seeking the information out themselves. In the second situation, the respondent also relied on her child to evaluate something that she believed had gone wrong. However, because these respondents took basic steps to protect themselves, they could not be considered to have high levels of reliance in all areas of security. Typically comments associated with moderate levels of reliance were focused on an area where the respondent considered themselves to be lacking. For example, not having enough knowledge to keep up with the threats meant that respondents relied on other people to tell them what they needed to be worried about. Similarly, not knowing how to fix problems meant that respondents sought out someone else to remedy breaches that had gotten past the security measures that the individual had taken.

Ranks of low to moderate and moderate to high were used to reconcile where respondents comments had features of multiple ranks. For example, a respondent who took steps to protect their computer and was happy with the steps they took, but who would seek someone out only if something "went wrong" would be considered to have low to moderate levels of reliance. In contrast, respondents who took basic protective steps but expected someone else to provide them with relevant security information and to fix any problems would be considered to have moderate to high levels of reliance. 

